\title{
Video Coding with Superimposed Motion-Compensated Signals
}

\author{
Videocodierung mit überlagerten \\ bewegungskompensierten Signalen
}

Der Technischen Fakultät der

Friedrich-Alexander-Universität Erlangen-Nürnberg

zur Erlangung des Grades

\section{Doktor-Ingenieur}

vorgelegt von

Markus Helmut Flierl

Erlangen, 2003 


\section{Als Dissertation genehmigt von der Technischen Fakultät der Friedrich-Alexander-Universität Erlangen-Nürnberg}

Tag der Einreichung: 13.03.2003

Tag der Promotion: 27.06.2003

Dekan: $\quad$ Prof. Dr. rer. Nat. A. Winnacker

Berichterstatter: Prof. Dr.-Ing. B. Girod

Prof. Dr.-Ing. A. Kaup 


\section{Acknowledgments}

This work required a substantial effort and I am very grateful to many people who made this endeavor possible. I would like to thank my supervisor Prof. Bernd Girod for the opportunity to join his group in Erlangen and Stanford as well as to benefit from the inspiring environment. I would also like to thank Prof. André Kaup for his interest in my work and reviewing my thesis as well as Prof. Heinrich Niemann and Prof. Wolfgang Koch for joining the committee. I am thankful to my fellow students Anne Aaron, Chuo-Ling Chang, Jacob Charareski, Joachim Eggers, Peter Eisert, Niko Färber, Sang-Eun Han, Frank Hartung, Mark Kalman, Yi Liang, Marcus Magnor, James Mammen, Prashant Ramanathan, Shantanu Rane, Marion Schabert, Eric Setton, Wolfgang Sörgel, Eckehard Steinbach, Klaus Stuhlmüller, Jonathan Su, Matthias Teschner, Lutz Trautmann, Thomas Wiegand, and Rui Zhang for many stimulating discussions, joint work, and proofreading. In addition, I am thankful for helpful discussions with Dr. Nick Kingsbury. I am also grateful to the people at the Telecommunications Laboratory and Information Systems Laboratory, especially to Ursula Arnold and Kelly Yilmaz for their invaluable administrative support. My special thanks belong to my family and friends for all the support they gave. 

Erkenne die Musik dessen vollendete Töne verklingen. Fühle die Stille die zum Träumen verführt. Erfahre den unendlichen Raum der hinschwindenden Ansammlung sich vollendender Töne.

- Deinem Odysseus 



\section{Contents}

1 Introduction 1

2 Background and Related Work $\quad 7$

2.1 Coding of Video Signals . . . . . . . . . . . . . . . . . . . . . 7

2.2 Motion Compensation . . . . . . . . . . . . . . . . . 8

2.2.1 Bidirectional Motion Compensation . . . . . . . . . . . . . . 9

2.2.2 Overlapped Block Motion Compensation . . . . . . . . . . . . . . . 10

2.2.3 Variable Block Size Motion Compensation . . . . . . . . . . . . . . 11

2.2.4 Multiframe Motion Compensation . . . . . . . . . . . . . . . . 12

2.2.5 Superimposed Motion Compensation . . . . . . . . . . . . . . . 13

2.3 Motion Estimation . . . . . . . . . . . . . . . . . . . . . 16

2.3.1 Rate-Constrained Motion Estimation . . . . . . . . . . . . . . 16

2.3.2 Rate-Constrained Estimation of Superimposed Motion . . . . . . . 18

2.3.3 Quantizer Selection at the Residual Encoder . . . . . . . . . . . . . 20

2.3.4 Efficient Motion Estimation _... . . . . . . . . . . . . . 22

2.4 Theory of Motion-Compensated Prediction . . . . . . . . . . . . . 23

2.4.1 Frame Signal Model . . . . . . . . . . . . . . . . . . . . . . . 24

2.4.2 Signal Model for Motion-Compensated Prediction . . . . . . . . . . 25

2.4.3 Signal Model for Multihypothesis Prediction . . . . . . . . . . . . 26

2.4.4 Performance Measures . . . . . . . . . . . . . . . . . . . . . 28

2.4 Conclusions . . . . . . . . . . . . . . . . . . . . . . . 29

2.5 Three-Dimensional Subband Coding of Video . . . . . . . . . . . . . . . . 29

2.5.1 Motion Compensation and Subband Coding . . . . . . . . . . . . 30

2.5.2 Motion-Compensated Lifted Wavelets . . . . . . . . . . . . . . . . 31 
3 Motion-Compensated Prediction with Complementary Hypotheses 33

3.1 Introduction . . . . . . . . . . . . . . . . . . 33

3.2 Extended Model for Superimposed Motion-Compensated Prediction . . . . 34

3.2.1 Superimposed Prediction and Correlated Displacement Error . . . . 34

3.2 .2 Complementary Hypotheses . . . . . . . . . . . . . . . . 36

3.2.3 Gradient of the Prediction Error Variance . . . . . . . . . . . . . . 41

3.3 Hypotheses with Additive Noise . . . . . . . . . . . . . . . . . . . . . . . 42

3.3.1 Averaging Filter . . . . . . . . . . . . . . . . . . 43

3.3 .2 Wiener Filter . . . . . . . . . . . . . . . . . . . 45

3.4 Forward-Adaptive Hypothesis Switching . . . . . . . . . . . . . . . . 52

3.4.1 Signal Model for Hypothesis Switching . . . . . . . . . . . . . . . . 53

3.4.2 Minimizing the Radial Displacement Error . . . . . . . . . . . . . 53

3.4 .3 Equivalent Predictor . . . . . . . . . . . . . . . 56

3.4.4 Motion Compensation with Complementary Hypotheses and Forward-Adaptive Hypothesis Switching . . . . . . . . . . . . . 57

3.5 Pictures with Varying Number of Hypotheses . . . . . . . . . . . . . . . 59

3.6 Conclusions . . . . . . . . . . . . . . . . . 61

4 ITU-T Rec. H.263 and Superimposed Prediction $\quad 63$

4.1 Introduction . . . . . . . . . . . . . . . . . . 63

4.2 Video Coding with Superimposed Motion . . . . . . . . . . . . . . . 64

4.2.1 Syntax Extensions . . . . . . . . . . . . . . . . . . . . 64

4.2 .2 Coder Control . . . . . . . . . . . . . . . . . . . . 64

4.3 Experimental Results . . . . . . . . . . . . . . . 66

4.3.1 Multiple Hypotheses for Constant Block Size . . . . . . . . . . . . 66

4.3.2 Multiple Hypotheses for Variable Block Size . . . . . . . . . . . . . 67

4.3.3 Multiple Hypotheses and Multiple Reference Pictures . . . . . . . . 69

4.4 Conclusions . . . . . . . . . . . . . . . . . . . 76

$5 \quad$ ITU-T Rec. H.264 and Generalized B-Pictures $\quad 77$

5.1 Introduction . . . . . . . . . . . . . . . . . 77

5.2 Prediction Modes for B-Pictures . . . . . . . . . . . . . . . . 78

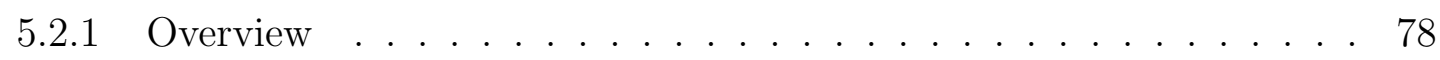

5.2 .2 Direct Mode . . . . . . . . . . . . . . . . . . . 79

5.2 .3 Superposition Mode . . . . . . . . . . . . . . . . . 80

5.2.4 Rate-Distortion Performance of Individual Modes . . . . . . . . . . 80

5.3 Superimposed Prediction . . . . . . . . . . . . . . . . 83

5.3.1 Bidirectional vs. Superposition Mode . . . . . . . . . . . . 83 
5.3.2 Two Combined Forward Prediction Signals . . . . . . . . . . . . . . 84

5.3.3 Entropy Coding . . . . . . . . . . . . . . . . . . . 89

5.4 Encoder Issues _ . . . . . . . . . . . . . . . . . . . . . . . . 90

5.4.1 Rate Constrained Mode Decision . . . . . . . . . . . . . . . 90

5.4.2 Rate Constrained Motion Estimation . . . . . . . . . . . . . . . 91

5.4.3 Rate Constrained Superimposed Motion Estimation . . . . . . . . . 92

5.4.4 Improving Overall Rate-Distortion Performance . . . . . . . . . . . 93

5.5 Conclusions . . . . . . . . . . . . . . . . . . . 97

6 Motion Compensation for Groups of Pictures 99

6.1 Introduction . . . . . . . . . . . . . . . . . . . . . . . . 99

6.2 Coding Scheme . . . . . . . . . . . . . . . . . . . . . . 99

6.2.1 Motion-Compensated Lifted Haar Wavelet . . . . . . . . . . . . . . 100

6.2.2 Motion-Compensated Lifted 5/3 Wavelet . . . . . . . . . . . . . . . 101

6.2.3 Experimental Results . . . . . . . . . . . . . . . . . . . . 101

6.3 A Mathematical Model of Motion-Compensated Subband Coding . . . . . 108

6.3.1 Motion-Compensated Lifted Haar Wavelet . . . . . . . . . . . . . . 108

6.3.2 Motion-Compensated Lifted 5/3 Wavelet . . . . . . . . . . . . . . 109

6.3.3 Signal Model . . . . . . . . . . . . . . . . . . . . . . . . . . 109

6.3.4 Transform Coding Gain . . . . . . . . . . . . . . . . . . . 113

6.4 Conclusions . . . . . . . . . . . . . . . . . . 115

$\begin{array}{lll}7 & \text { Summary } & 117\end{array}$

A Mathematical Results $\quad 123$

A.1 Singularities of the Displacement Error Covariance Matrix . . . . . . . . . 123

A.2 A Class of Matrices and their Eigenvalues . . . . . . . . . . . . . . . 123

A.3 Inverse of the Power Spectral Density Matrix . . . . . . . . . . . . . . . . . 124

A.4 Power Spectral Density of a Frame . . . . . . . . . . . . . . 126

$\begin{array}{ll}\text { Glossary } & 127\end{array}$

$\begin{array}{ll}\text { Bibliography } & 131\end{array}$ 


\section{Inhaltsverzeichnis ${ }^{1}$}

1 Einführung 1

2 Grundlagen und Stand der Forschung $\quad 7$

2.1 Codierung von Videosignalen . . . . . . . . . . . . . . . 7

2.2 Bewegungskompensation . . . . . . . . . . . . . . . . 8

2.2.1 Bidirektionale Bewegungskompensation . . . . . . . . . . . . . 9

2.2.2 Bewegungskompensation mit überlappenden Blöcken . . . . . . . . 10

2.2.3 Bewegungskompensation mit Blöcken variabler Größe . . . . . . . . 11

2.2.4 Bewegungskompensation mit mehreren Referenzbildern . . . . . . . 12

2.2.5 Überlagerte Bewegungskompensation . . . . . . . . . . . . . . . . . 13

2.3 Bewegungsschätzung . . . . . . . . . . . . . . . . . 16

2.3.1 Bewegungsschätzung mit Ratennebenbedingung . . . . . . . . . 16

2.3.2 Schätzung überlagerter Bewegung mit Ratennebenbedingung . . . . 18

2.3.3 Wahl des Quantisierers beim Prädiktionsfehler-Encoder . . . . . . . 20

2.3.4 Effiziente Bewegungsschätzung . . . . . . . . . . . . . . . . 22

2.4 Theorie der bewegungskompensierten Prädiktion . . . . . . . . . . . . 23

2.4.1 Bildsignalmodell . . . . . . . . . . . . . . . . . . . . . 24

2.4.2 Signalmodell für bewegungskompensierte Prädiktion . . . . . . . . . 25

2.4.3 Signalmodell für Multihypothesen-Prädiktion . . . . . . . . . . 26

2.4.4 Bewertungsmaße . . . . . . . . . . . . . . . . . . . . . 28

2.4.5 Schlussfolgerungen . . . . . . . . . . . . . . . . . . . 29

2.5 Drei-Dimensionale Teilbandcodierung von Video . . . . . . . . . . . . . . 29

2.5.1 Bewegungskompensation und Teilbandcodierung . . . . . . . . . . . 30

2.5.2 Bewegungskompensierte Wavelets . . . . . . . . . . . . . . . 31

3 Bewegungskompensierte Prädiktion mit komplementären Hypothesen 33

3.1 Einleitung . . . . . . . . . . . . . . . . . . . . . 33

3.2 Erweitertes Modell für die überlagerte bewegungskompensierte Prädiktion . 34

3.2.1 Überlagerte Prädiktion und korrelierte Versatzfehler . . . . . . . . . . 34

3.2 .2 Komplementäre Hypothesen . . . . . . . . . . . . . . . . . . . . . . 36

3.2.3 Gradient der Prädiktionsfehlervarianz . . . . . . . . . . . . . . . . . 41

3.3 Hypothesen mit additivem Rauschen . . . . . . . . . . . . . . . . . . . . . 42

3.3.1 Mittelungsfilter . . . . . . . . . . . . . . . . . 43

3.3.2 Wiener Filter . . . . . . . . . . . . . . . . . . . 45

\footnotetext{
${ }^{1}$ This translation of the table of contents is a requirement according to the "Prüfungsordnung für die Technischen Fakultät der Friedrich-Alexander-Universität Erlangen-Nürnberg".
} 
3.4 Adaptive Hypothesenauswahl . . . . . . . . . . . . . . . . . . . . 52

3.4.1 Signalmodell für die Auswahl von Hypothesen . . . . . . . . . . . . 53

3.4.2 Minimierung des radialen Versatzfehlers . . . . . . . . . . . . . 53

3.4 .3 Äquivalenter Prädiktor . . . . . . . . . . . . . . . . . . . 56

3.4.4 Bewegungskompensation mit komplementären und adaptiv ausgewählten Hypothesen . . . . . . . . . . . . . . . . . . 57

3.5 Bilder mit variierender Anzahl an Hypothesen . . . . . . . . . . . . . . . . 59

3.6 Schlussfolgerungen . . . . . . . . . . . . . . . . . . 61

4 ITU-T Rec. H.263 und überlagerte Prädiktion 63

4.1 Einleitung . . . . . . . . . . . . . . . . . . 63

4.2 Videocodierung mit überlagerter Bewegung . . . . . . . . . . . . . . 64

4.2.1 Syntax Erweiterung . . . . . . . . . . . . . . . . . 64

4.2.2 Algorithmen für den Encoder . . . . . . . . . . . . . . . . . . . 64

4.3 Experimentelle Ergebnisse . . . . . . . . . . . . . . . . 66

4.3.1 Konstante Blockgröße und mehrere Hypothesen . . . . . . . . . . . 66

4.3.2 Variable Blockgröße und mehrere Hypothesen . . . . . . . . . . . . 67

4.3.3 Mehrere Hypothesen und mehrere Referenzbilder . . . . . . . . . . 69

4.4 Schlussfolgerungen . . . . . . . . . . . . . . . . . 76

5 ITU-T Rec. H.264 und verallgemeinerte B-Bilder $\quad 77$

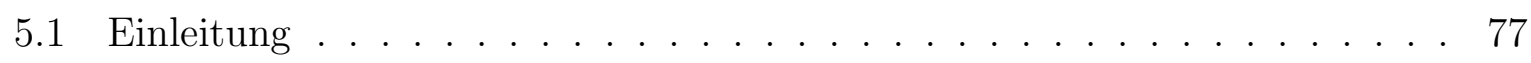

5.2 Prädiktionsmodi für B-Bilder . . . . . . . . . . . . . . . . . . 78

5.2 .1 Überblick . . . . . . . . . . . . . . . . . . . . . . . . . . 78

5.2 .2 Direct Mode . . . . . . . . . . . . . . . . . . . . 79

5.2 .3 Überlagernder Mode . . . . . . . . . . . . . . . . . . . . . . . 80

5.2.4 Kompressionseffizienz einzelner Modi . . . . . . . . . . . . . . . 80

5.3 Überlagerte Prädiktion . . . . . . . . . . . . . . . . . . . . . . . . 83

5.3.1 Bidirektionaler vs. Überlagernder Mode . . . . . . . . . . . . . . . 83

5.3.2 Lineare Überlagerung zweier vorwärts-prädizierter Signale . . . . . 84

5.3 .3 Entropiecodierung . . . . . . . . . . . . . . . . 89

5.4 Anmerkungen zum Encoder . . . . . . . . . . . . . . . . . 90

5.4.1 Modeentscheidung mit Ratennebenbedingung . . . . . . . . . . . . 90

5.4 .2 Bewegungsschätzung mit Ratennebenbedingung . . . . . . . . . . . 91

5.4.3 Bewegungsschätzung mit Ratennebenbedingung für überlagerte Signale . . . . . . . . . . . . . . . . . . . . . . . 92

5.4.4 Verbesserung der Gesamteffizienz ... . . . . . . . . . 93

5.5 Schlussfolgerungen . . . . . . . . . . . . . . . . . . . . 97 
6 Bewegungskompensation für Gruppen von Bildern 99

6.1 Einleitung . . . . . . . . . . . . . . . . . . . . . . . . . . . 99

6.2 Codieralgorithmus . . . . . . . . . . . . . . . . . . . . 99

6.2.1 Haar Wavelet mit Bewegungskompensation . . . . . . . . . . . . . . 100

6.2.2 5/3 Wavelet mit Bewegungskompensation . . . . . . . . . . . . . 101

6.2.3 Experimentelle Ergebnisse . . . . . . . . . . . . . . . . . . . 101

6.3 Mathematisches Modell der bewegungskompensierten Teilbandcodierung . 108

6.3.1 Haar Wavelet mit Bewegungskompensation . . . . . . . . . . . . . . 108

$6.3 .25 / 3$ Wavelet mit Bewegungskompensation . . . . . . . . . . . . . . 109

6.3.3 Signalmodell . . . . . . . . . . . . . . . . . . . . . . . 109

6.3.4 Codiergewinn der Transformation . . . . . . . . . . . . . . . 113

6.4 Schlussfolgerungen . . . . . . . . . . . . . . . . . . . . . 115

$\begin{array}{lll}7 & \text { Zusammenfassung } & 117\end{array}$

$\begin{array}{ll}\text { A Mathematische Ergebnisse } & 123\end{array}$

A.1 Singularitäten der Kovarianzmatrix . . . . . . . . . . . . . . . . . 123

A.2 Eine Klasse von Matrizen und ihre Eigenwerte . . . . . . . . . . . . . . . . 123

A.3 Inverse Matrix der Leistungsdichtespektren . . . . . . . . . . . . . . . . 124

A.4 Leistungsdichtespektrum eines Bildes . . . . . . . . . . . . . . . . . 126

$\begin{array}{ll}\text { Abkürzungen und Formelzeichen } & 127\end{array}$

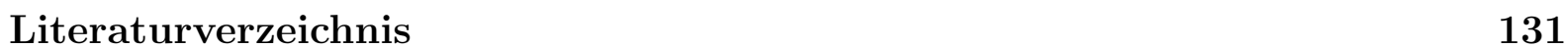




\section{Introduction}

Motion-compensated prediction is an important component of current hybrid video coding systems. In recent years, advances in compression efficiency have mainly been achieved by improved motion-compensated prediction, e.g. sub-pel accurate motion compensation [Gir93a], variable block size prediction [SB91b], multiframe prediction [GV93, BG96, WZG97a], and multihypothesis motion compensation.

Multihypothesis motion-compensated prediction linearly combines multiple motioncompensated signals to arrive at the actual prediction signal. This term was first used in [Sul93] to provide a framework for overlapped block motion compensation (OBMC). OBMC was introduced to reduce blocking artifacts in motion-compensated prediction [NO92]. In earlier work, attempts have been made to combine signals from more than one frame. Published in 1985, [Eri85] investigates adaptive predictors for hybrid coding that use up to four previous fields. In the same year, the efficiency of bidirectional prediction has been raised in [MPG85]. To predict the current frame, bidirectional prediction uses a linear combination of two motion-compensated signals: one is chosen from the next reference frame, the other from the previous reference frame. Bidirectional prediction characterizes the now known concept of B-pictures which has originally been proposed to MPEG [PAHL90]. The motivation was to interpolate any skipped frame taking into account the movement between the two "end" frames. The technique, originally called conditional motion-compensated interpolation, coupled the motion-compensated interpolation strategy with transmission of significant interpolation errors.

These practical schemes have been studied in [Gir00] and summarized in a theoretical analysis of multihypothesis motion-compensated prediction. The analysis is based on a power spectral density model for inaccurate motion compensation [Gir87] which has been proven successful to characterize motion-compensated prediction. Variations of these practical schemes have also been standardized in, e.g., [ISO96] and [ITU00].

With the advent of multiframe prediction, the question of linearly combined prediction signals has been re-visited. [Fli97a, FWG98] design general predictors for block-based superimposed motion compensation that utilize several previous reference frames. To determine an efficient set of motion-compensated blocks, an iterative algorithm is used to improve successively conditional optimal motion-compensated blocks. A similar algorithm has been proposed in [WG94] for bidirectional prediction only.

Multiple frames are not only used for predictive coding. Schemes for three-dimensional subband coding of video signals consider also multiple frames [Kna76, Kro89]. Adaptive wavelet transforms with motion compensation can be used for temporal subband decomposition [ST01]. These schemes use again linear combinations of motion-compensated signals and are also of interest for our investigations. 
This thesis "Video Coding with Superimposed Motion-Compensated Signals", contributes to the field of motion-compensated video coding as follows:

1. For video compression, we investigate the efficiency of block-based superimposed prediction with multiframe motion compensation based on the ITU-T Rec. H.263. We explore the efficient number of superimposed prediction signals, the impact of variable block sizes compensation, and the influence of the size of the multiple reference frame buffer if the reference frames are previous frames only.

2. We generalize B-pictures for the emerging ITU-T Rec. H.264 to the generic concept of superimposed prediction which chooses motion-compensated blocks from an arbitrary set of reference pictures and measure the improvement in compression efficiency. Further, the generic concept of superimposed prediction allows also that generalized B-pictures are used for reference to predict other B-pictures. As this is not the case for classic B-pictures, we explore the efficiency of this aspect too.

3. We build on the theory of multihypothesis motion-compensated prediction for video coding and extend it to motion-compensated prediction with complementary hypotheses. We assume that the displacement errors of the multiple hypotheses are jointly distributed and, in particular, correlated. We investigate the efficiency of superimposed motion compensation as a function of the displacement error correlation coefficient. We conclude that compensation with complementary hypotheses results in maximally negatively correlated displacement error. We continue and determine a high-rate approximation for the rate difference with respect to optimal intra-frame encoding and compare the results to [Gir00]. To capture the influence of multiframe motion compensation, we model it by forward-adaptive hypothesis switching and show that switching among $M$ hypotheses with statistically independent displacement error reduces the displacement error variance by up to a factor of $M$.

4. We do not only consider predictive coding with motion compensation. We explore also the combination of linear transforms and motion compensation for a temporal subband decomposition of video and discuss motion compensation for groups of pictures. Therefore, we investigate experimentally and theoretically motion-compensated lifted wavelets for three-dimensional subband coding of video. The experiments provide insight about the coding efficiency depending on the number of pictures in the group and permit a comparison to predictive coding with motion compensation. The theoretical discussion analyzes the investigated lifted wavelets and builds on the insights from motion compensation with multiple hypotheses. Further, the analysis provides performance bounds for three-dimensional subband coding with motion compensation and gives insight about potential coding gains.

This thesis is organized as follows: Chapter 2 provides the background for video coding with superimposed motion-compensated signals and discusses related work. Chapter 3 investigates motion-compensated prediction with complementary hypotheses. Based on 
ITU-T Rec. H.263, Chapter 4 explores experimental results for video coding with superimposed motion-compensated prediction and multiple reference frames. Chapter 5 discusses generalized B-pictures for the emerging ITU-T Rec. H.264. Finally, Chapter 6 explores linear transforms with motion compensation and its application to motion compensation for groups of pictures.

\section{Einleitung ${ }^{1}$}

Bewegungskompensierte Prädiktion ist eine wichtige Komponente heutiger hybrider Videocodiersysteme. In den letzten Jahren wurden Fortschritte bei der Kompressionseffizienz hauptsächlich durch Verbesserung der bewegungskompensierten Prädiktion erzielt, z.B. sub-pel genaue Bewegungskompensation [Gir93a], Prädiktion mit variabler Blockgröße [SB91b], Prädiktion mit mehreren Referenzbildern [GV93, BG96, WZG97a] und bewegungskompensierte Multihypothesen-Prädiktion.

Für die bewegungskompensierte Multihypothesen-Prädiktion werden mehrere bewegungskompensierte Signale linear überlagert um das endgültige Prädiktionssignal zu erhalten. Der Begriff wurde erstmals in [Sul93] verwendet um Bewegungskompensation mit überlappenden Blöcken (OBMC) zu beschreiben. OBMC wurde eingeführt um BlockArtefakte bei der bewegungskompensierten Prädiktion zu reduzieren [NO92]. Jedoch wurde bereits 1985 ein adaptiver Prädiktor für die hybride Videocodierung untersucht, der bis zu vier vorangehende Felder verwendet [Eri85]. Im selben Jahr wurde auch die Effizienz der bidirektionalen Prädiktion hinterfragt [MPG85]. Bidirektionale Prädiktion verwendet eine Linearkombination zweier bewegungskompensierter Signale um das momentane Bild zu prädizieren. Dabei wird ein Signal vom folgenden, das andere vom vorangehenden Referenzbild gewählt. Bidirektionale Prädiktion charakterisiert das heute bekannte Konzept der B-Bilder, das ursprünglich für die MPEG Standardisierung vorgeschlagen wurde [PAHL90]. Um ausgelassene Bilder besser zu interpolieren, wurde die Bewegung zwischen den "einschließenden" Bildern berücksichtigt. Die Methode, die ursprünglich bedingte bewegungskompensierte Interpolation hieß, verband das Konzept der bewegungskompensierten Interpolation mit der Übertragung signifikanter Interpolationsfehler.

Diese praktischen Verfahren wurden in [Gir00] untersucht und mündeten in eine theoretische Analyse der bewegungskompensierten Multihypothesen-Prädiktion. Die Analyse basiert auf einem statistischen Modell zweiter Ordnung und quantifiziert Effizienzeinbußen durch ungenaue Bewegungskompensation [Gir87]. Dieses statistische Modell eignet sich sehr gut um bewegungskompensierte Prädiktion zu beschreiben. Varianten dieser praktischen Verfahren wurden zum Beispiel in [ISO96] und [ITU00] standardisiert.

Durch die Einführung der Prädiktion mit mehreren Referenzbildern (Multiframe-Prädiktion) wurde die Frage nach linear überlagerten Prädiktionssignalen wieder aufgeworfen. Die Arbeit in [Fli97a, FWG98] entwirft allgemeine Prädiktoren für blockbasierte,

\footnotetext{
${ }^{1}$ This translation of the introduction is a requirement according to the "Prüfungsordnung für die Technischen Fakultät der Friedrich-Alexander-Universität Erlangen-Nürnberg".
} 
überlagerte Bewegungskompensation mit mehreren vorangehenden Referenzbildern. Es wird ein iterativer Algorithmus verwendet der sukzessiv bedingt-optimale, bewegungskompensierte Blöcke verbessert um effiziente Block-Tupel zu bestimmen. Ein ähnlicher Algorithmus wurde nur für die bidirektionale Prädiktion in [WG94] vorgeschlagen.

Mehrere Bilder werden nicht nur für die prädiktive Codierung herangezogen. Verfahren für drei-dimensionale Teilbandcodierung von Videosignalen berücksichtigen auch mehrere Bilder [Kna76, Kro89]. Adaptive Wavelet-Transformationen mit Bewegungskompensation können für die zeitliche Teilbandzerlegung verwendet werden [ST01]. Diese Verfahren überlagern wiederum bewegungskompensierte Signale und sind für unsere Untersuchungen ebenfalls von Interesse.

Die vorliegende Dissertationsschrift "Videocodierung mit überlagerten bewegungskompensierten Signalen" trägt zu dem Gebiet der bewegungskompensierten Videocodierung wie folgt bei:

1. Mit Hilfe der ITU-T Rec. H.263 wird die Kompressionseffizienz der blockbasierten, überlagerten Bewegungskompensation mit mehreren Referenzbildern untersucht. Es wird die Anzahl der überlagerten bewegungskompensierten Signale, die Einwirkung der Bewegungskompensation mit Blöcken variabler Größe und der Einfluss der Größe des Referenzbildspeichers für den Fall untersucht, dass sich nur vorangehende Referenzbilder im Speicher befinden.

2. Weiterhin wird das B-Bild für die neue ITU-T Rec. H.264 verallgemeinert und die Verbesserung der Kompressionseffizienz quantifiziert. Die Verallgemeinerung realisiert das generische Konzept der bewegungskompensierten Prädiktion mit überlagerten Blöcken, die aus einer beliebigen Referenzbildmenge herrühren. Das generische Konzept der überlagerten Prädiktion erlaubt weiterhin, dass verallgemeinerte B-Bilder als Referenz herangezogen werden um andere B-Bilder zu prädizieren. Da dies nicht für klassische B-Bilder zutrifft, wird dieser Aspekt ebenfalls untersucht.

3. Basierend auf der Theorie der bewegungskompensierten Multihypothesen-Prädiktion wird das Konzept der Prädiktion mit komplementären bewegungskompensierten Signalen eingeführt. Es wird vorgeschlagen, dass die Versatzfehler der bewegungskompensierten Signale eine gemeinsame Wahrscheinlichkeitsverteilung aufweisen und, im Besonderen, korreliert sind. Die Effizienz der Prädiktion mit überlagerten bewegungskompensierten Signalen wird in Abhängigkeit des Versatzfehler-Korrelationskoeffizienten untersucht. Es wird festgestellt, dass die Prädiktion mit komplementären bewegungskompensierten Signalen zu maximal negativ-korrelierten Versatzfehlern führt. Eine Hochraten-Approximation für die Ratendifferenz bezüglich optimaler Intra-Bild Codierung erlaubt einen Vergleich mit den Ergebnissen in [Gir00]. Um den Einfluss der Bewegungskompensation mit mehreren Referenzbildern zu erfassen, wird das Verfahren durch eine vorwärts-adaptive Auswahl der bewegungskompensierten Signale modelliert. Es wird gezeigt, dass die Auswahl unter $M$ bewegungskompensierten Signalen mit statistisch unabhängigen Versatzfehlern die Versatzfehlervarianz höchstens um den Faktor $M$ reduziert. 
4. Die vorliegende Arbeit untersucht nicht nur prädiktive Codierung mit Bewegungskompensation. Es wird auch die Kombination von linearer Transformation und Bewegungskompensation für die Teilbandzerlegung von Video erforscht und die Bewegungskompensation für Gruppen von Bildern diskutiert. So werden bewegungskompensierte Wavelets für die drei-dimensionale Teilbandcodierung von Video experimentell und theoretisch untersucht. Die Experimente geben Aufschluss über die Kompressionseffizienz in Abhängigkeit von der Anzahl der Bilder in einer Gruppe und erlauben einen Vergleich zur prädiktiven Codierung mit Bewegungskompensation. Die theoretische Diskussion analysiert die untersuchten Wavelets und baut auf die Erkenntnisse, die für die Prädiktion mit überlagerten bewegungskompensierten Signalen erzielt wurden. Die Analyse gibt einerseits Aufschluss über die Grenzen der Effizienz der drei-dimensionalen Teilbandcodierung mit Bewegungskompensation sowie andererseits Einblick in mögliche Codiergewinne.

Die vorliegende Arbeit ist wie folgt gegliedert: Kapitel 2 diskutiert relevante und artverwandte Vorarbeiten zur Videocodierung mit überlagerten bewegungskompensierten Signalen. Kapitel 3 untersucht die Prädiktion mit komplementären bewegungskompensierten Signalen. Basierend auf ITU-T Rec. H.263 beschreibt Kapitel 4 experimentelle Ergebnisse für die Videocodierung mit überlagerter bewegungskompensierter Prädiktion und mehreren Referenzbildern. Anschließend diskutiert Kapitel 5 verallgemeinerte B-Bilder für die neue ITU-T Rec. H.264. Schließlich untersucht Kapitel 6 lineare Transformationen mit Bewegungskompensation und ihre Anwendung bei der Bewegungskompensation für Gruppen von Bildern. 


\section{Background and Related Work}

\subsection{Coding of Video Signals}

Standard video codecs like ITU-T Recommendation H.263 [ITU00, CEGK98] or the emerging ITU-T Recommendation H.264 [ITU02] are hybrid video codecs. They incorporate an intra-frame codec and a motion-compensated predictor. The intra-frame codec is able to encode and decode one frame independently of others, whereas the motioncompensated predictor is able to compensate motion between the current frame and a previously decoded frame.

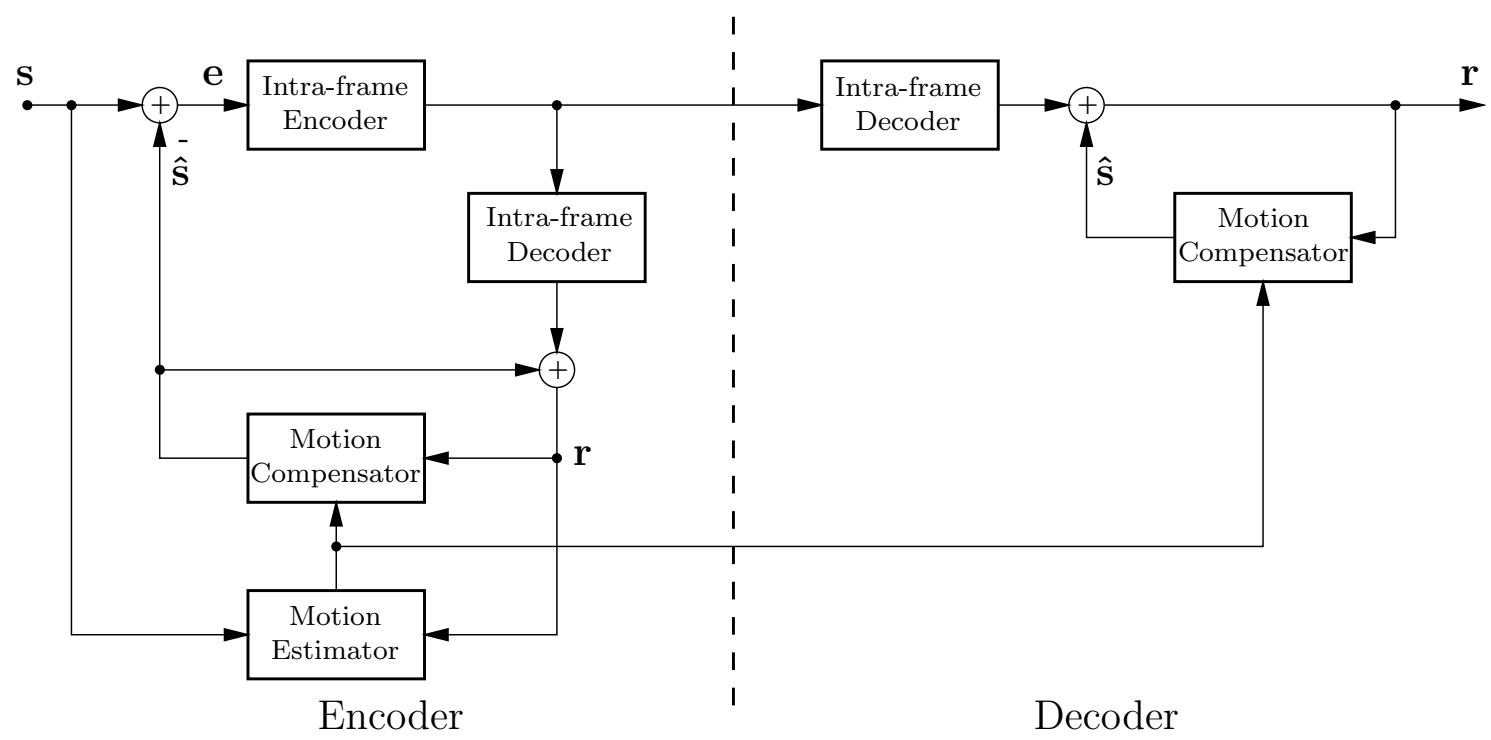

Figure 2.1: Hybrid video codec utilizing motion-compensated prediction.

Fig. 2.1 depicts such a hybrid video codec. The encoder estimates the motion between the current frame $\mathbf{s}$ and a previously decoded frame $\mathbf{r}$ and transmits it as side-information to the decoder. Both encoder and decoder use motion compensation to generate the motion-compensated frame $\hat{\mathbf{s}}$ from previously reconstructed frames which are also available at the decoder. Therefore, only the frame difference e between the current frame and the motion-compensated frame needs to be encoded by the intra-frame encoder. This frame difference has much less signal energy than the current frame and, hence, requires less bit-rate if encoded. Despite the side-information, the overall bit-rate of a hybrid video codec is less than the bit-rate of a video codec with intra-frame coding only. Therefore, motion-compensated prediction is an important component for efficient compression with a hybrid video codec. 
Hybrid video codecs require sequential processing of video signals which makes it difficult to achieve efficient embedded representations of video sequences. Therefore, we consider also motion-compensated three-dimensional subband coding of video signals [TZ94b, Ohm94b, CW99]. Applying a linear transform in temporal direction of a video sequence may not be very efficient if significant motion is prevalent. Motion compensation between two frames is necessary to deal with the motion in a sequence. A combination of linear transform and motion compensation is required for efficient three-dimensional subband coding.

In the following, we review relevant techniques and principles for state-of-the-art video coding. The discussion provides a background for the following chapters and summarizes work on which we will build. Section 2.2 outlines several relevant methods for motioncompensation: bidirectional motion compensation, overlapped block motion compensation, variable block size motion compensation, multiframe motion compensation, and superimposed motion compensation. Section 2.3 discusses previous work on rate-constrained motion estimation, rate-constrained estimation of superimposed motion, quantizer selection at the residual encoder, and techniques for efficient motion estimation. Section 2.4 introduces to a theory for motion-compensated prediction. We discuss the underlying frame signal model, review the model for motion-compensated prediction, and outline the state-of-the-art for multihypothesis motion-compensated prediction. We reference the utilized performance measures and summarize the results of this theory. Finally, Section 2.5 summarizes previous work on three-dimensional subband coding of video. We outline the problem of motion compensation for the temporal subband decomposition and refer to adaptive lifting schemes that permit motion compensation.

\subsection{Motion Compensation}

The efficiency of inter-frame coding schemes for video sequences is improved by motion compensation. Efficient motion compensation requires an accurate measurement of the displacement field between two frames. A practical algorithm for this measurement is block matching [JJ81] [NO82]. It estimates the displacement field on a block bases, i.e., approximates each block with one displacement value by minimizing its prediction error. Efficient motion-compensated coding is desirable especially for low bit-rate video applications [Pur88]. Usually, block-based schemes assume just translatory motion for all pels in the block. But more sophisticated schemes like spatial transforms [NH94] and transformed block-based motion compensation [LF95] are possible. And by omitting the block constraint, arbitrarily shaped regions can be used for motion compensation [YMO95].

Efficient inter-frame coding schemes consider also the problem of noise reduction in image sequences. DUBOIS and SABRI describe in [DS84] a nonlinear temporal filtering algorithm using motion compensation. Woods et al. present a spatio-temporal adaptive 3-D Kalman filter with motion compensation [KW97] and couple it with motion estimation in [KW98]. 


\subsubsection{Bidirectional Motion Compensation}

Frame skipping is a viable technique to reduce drastically the bit-rate necessary to transmit a video signal. If all frames have to be reconstructed at the decoder, skipped frames must be interpolated by a motion compensation scheme. NETRAVALI and RoBBINS propose such a scheme in [NR83] and initiate further research in this field. SoRYANI and CLARKE combine image segmentation and adaptive frame interpolation [SC89], Thoma and BierLing consider covered and uncovered background for motioncompensated interpolation [TB89], and CAFFORIO et al. discuss a pel-recursive algorithm [CRT90]. Similar algorithms for adaptive frame interpolation are outlined in [RCS92, ZZ93, Cas96, FVC97]. KovaČEvić et al. use adaptive bidirectional interpolation for deinterlacing [KSY95, KSY97]. Au et al. study also temporal frame interpolation [WA96] and compare block- and pixel-based interpolation in [TA98]. They propose temporal interpolation with overlapping [WAT96] and unidirectional motion-compensated temporal interpolation [TA97]. They also suggest zonal based algorithms for temporal interpolation [TCLA01]. These algorithms allow efficient block-based motion estimation [TCAL01, TAL02].

To improve the quality of the interpolated frames at the decoder, the interpolation error is encoded and additional bits are transmitted to the decoder. MICKE considers first the idea of interpolation error encoding in [Mic86]. HASKELL and PURI [HP90] as well as Yonemitsu and ANDREws [YA92] propose algorithms for this approach. This type of coded picture is also called B-picture. PURI et al. investigate several aspects of this picture type, like quantization and temporal resolution scalability [PA90, PA91, PAH93, PYH94]. Additionally, Shanablem and GHAnBARI point out the importance of B-pictures in video streaming [SG01].

LYNCH shows how to derive B-picture motion vectors from neighboring P-pictures [Lyn92]. But the displacement field of the B-pictures can also be encoded and transmitted to the decoder. For example, Woods et al. suggest compactly encoded optical-flow fields and label fields for frame interpolation and bidirectional prediction [KWM99].

B-pictures, as they are employed in MPEG-1 [Tek95], MPEG-2 [ISO96], or H.263 [ITU98a], utilize Bidirectional Motion Compensation. Bidirectional motion-compensated prediction is an example for multihypothesis motion-compensated prediction where two motion-compensated signals are superimposed to reduce the bit-rate of a video codec. But the concept of B-pictures has to deal with a significant drawback: prediction uses the reference pictures before and after the B-pictures as depicted in Fig. 2.2. The associated delay of several frames may be unacceptable for interactive applications. In addition, the two motion vectors always point to the previous and subsequent frames and the advantage of a variable picture reference cannot be exploited.

The efficiency of forward and backward prediction has already been raised by MusMANN et al. [MPG85] in 1985. The now known concept of B-pictures was proposed to MPEG by PURI et al. [PAHL90]. The motivation was to interpolate any skipped frame taking into account the movement between the two "end" frames. The technique, called 


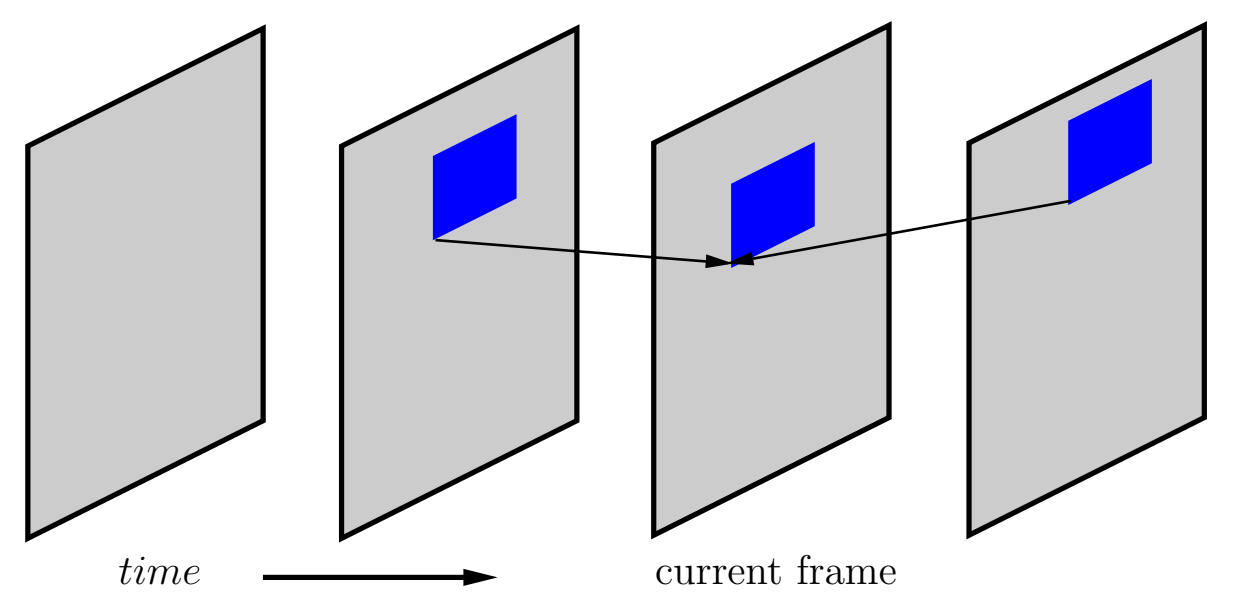

Figure 2.2: Bidirectional motion compensation. Reference pictures are used only before and after the current frame.

conditional motion-compensated interpolation, couples the motion-compensated interpolation strategy with transmission of the significant interpolation errors.

For joint estimation of forward and backward motion vectors in bidirectional prediction, a low-complexity iterative algorithm is introduced in [Wu93]. Starting from the initial values obtained by a commonly-used block matching independent search method, the motion vectors are iteratively refined until a locally optimal solution to the motion estimation problem for bidirectional prediction is achieved. Each iteration consists of a series of two similar procedures. First, the backward motion vector is fixed and a new forward motion vector is searched to minimize the prediction error. Then the forward motion vector is fixed and the backward motion vector is similarly refined by minimizing the prediction error. This process is repeated until the prediction error no further decreases. The iterative search procedure minimizes the prediction error and considers no rate constraint [WG94]. The price paid for the improvement in performance is only a small increase in computational complexity relative to independent search for the two motion vectors. Experiments in [WG94] show that the increase in search complexity is, on average, less than $20 \%$ of that of the independent search. Based on this work, [KK94] proposes an efficient motion estimation algorithm.

\subsubsection{Overlapped Block Motion Compensation}

Like bidirectional prediction, Overlapped Block Motion Compensation (OBMC) [NO92, Sul93, SO94, OS94, KO95] is another example of the general concept of multihypothesis motion-compensated prediction. Originally, the motivation was to reduce blocking artifacts caused by block motion compensation. SULLIVAN and BAKER introduced motion compensation using control grid interpolation [SB91a] and WATANABE and SinghaL windowed motion compensation [WS91]. OBMC uses more than one motion vector for predicting the same pixel but, in contrast to bidirectional prediction, does not increase 
the number of vectors per block.

The discussion of overlapped compensation in [Sul93] is based on 'multi-hypothesis expectation'. The paper argues that only one block motion vector is encoded for a large block of pixels and that the vector value is limited in precision such that an encoded block motion vector may not be correct for all pixels in the block. For this reason, [Sul93] proposes a multi-hypothesis expectation paradigm. Since the decoder cannot know the correct motion vector for each pixel, the motion uncertainty is modeled by a posteriori inferred displacement probability density function, conditioned on the encoded data. Using this distribution, an ideal decoder could generate a minimum mean square error estimate for each pixel prediction. A vector of linear weights and an associated set of displacements are defined to determine the prediction for each pixel. It is reported that the method effectively eliminates blocking artifacts and reduces prediction error.

[OS94] proposes an estimation-theoretic paradigm for analyzing and optimizing the performance of block-based motion compensation algorithms. OBMC is derived as a linear estimator of each pixel intensity, given that the only motion information available to the decoder is a set of block-based vectors. OBMC predicts the frame of a sequence by repositioning overlapping blocks of pixels from the reference frame, each weighted by some smooth window. The estimation-theoretic formulation leads directly to statistical techniques for optimized window design. The work also considers the problem of optimized motion estimation. Viewed within the estimation-theoretic paradigm for blockbased motion compensation, the objective of motion estimation is to provide the decoder information that optimizes the performance of its prediction. Optimal motion estimation for OBMC involves estimating a noncausally related motion field, and an iterative procedure is proposed for solving this problem.

TAO and ORCHARD continue the investigation. With the goal to remove motion uncertainty and quantization noise [TO01c], they discuss OBMC and loop filtering in [TOD97], propose a method for window design [TO97], and investigate non-iterative motion estimation [TO98]. They also suggest a gradient-based model for the residual variance [TO01a] and propose a parametric solution for OBMC [TO01b].

\subsubsection{Variable Block Size Motion Compensation}

Motion-compensated prediction with blocks of variable size improves the efficiency of video compression algorithms by adapting spatially displacement information [Str90, SB91b, SB94, Lee95, RCN97, Fli97b, WFG97]. Variable Block Size (VBS) prediction assigns more than one motion vector per macroblock but it uses just one motion vector for a particular pixel.

[SB91b] describes a method for optimizing the performance of block-based motioncompensated prediction for video compression using fixed or variable size blocks. A Lagrangian cost function is used to choose motion vectors and block sizes for each region of the prediction image, that gives the best performance in a rate-distortion sense. For 
that, a quadtree is used to structure blocks of variable size. The variable block size algorithm determines the optimal tree structure and yields a significant improvement in rate-distortion performance over motion compensation with a fixed block size.

[Fli97b, WFG97] investigate a more general tree structure for motion- and intensitycompensated video coding. In contrast to variable block size motion compensation, this approach incorporates also the intensity residual into the tree structure. The work discusses pruning and growing algorithms to determine rate-distortion optimal tree structures by utilizing a Lagrangian cost function.

ITU-T Rec. H.263 [ITU00] provides variable block size capability. The INTER4V coding mode allows $8 \times 8$ blocks in addition to the standard $16 \times 16$ blocks. VBS motioncompensated prediction utilizes either OBMC or an in-loop deblocking filter to reduce blocking artifacts. The emerging ITU-T Rec. H.264 [ITU02] allows up to 7 different block sizes from $16 \times 16$ to $4 \times 4$. Here, the blocking artifacts are reduced by an in-loop deblocking filter.

\subsubsection{Multiframe Motion Compensation}

Multiframe techniques have first been utilized for background prediction by MuKAwA and KuRODA [MK85]. The method permits a prediction signal for uncovered background. With a special background memory, the method is also investigated by HEPPER [Hep90]. LAVAGETTO and LEONARDI discuss block-adaptive quantization of multiple-frame motion fields [LL91]. GoTHE and VAISEY improve motion compensation by using multiple temporal frames [GV93]. They provide experimental results for $8 \times 8$ block-motion compensation with up to 8 previous frames. Fukuhara, Asai, and Murakami propose low bit-rate video coding with block partitioning and adaptive selection of two time-differential frame memories [FAM97]. The concept of multiframe motion-compensated prediction is used by BUdAGAVI and GiBson to control error propagation for video transmission over wireless channels [BG96, BG97, BG98, BG01].

Long-term memory motion-compensated prediction [WZG97a, WZG97b, WZG99, Wie00, WG01b] employs several reference frames, i.e., several previously decoded frames, whereas standard motion-compensated prediction utilizes one reference frame, i.e. the previously decoded frame. This is accomplished by assigning a variable picture reference parameter to each block motion vector as shown in Fig. 2.3. The additional reference parameter overcomes the restriction that a specific block has to be chosen from a certain reference frame. This generalization improves compression efficiency of motion-compensated prediction.

In [WZG99], the long-term memory covers several seconds of decoded frames at the encoder and decoder. The use of multiple frames for motion compensation in most cases provides significantly improved prediction gain. The variable picture reference that permits the use of several frames has to be transmitted as side information requiring an additional bit-rate which may be prohibitive when the size of the long-term memory becomes large. Therefore, the bit-rate of the motion information is controlled by employing 


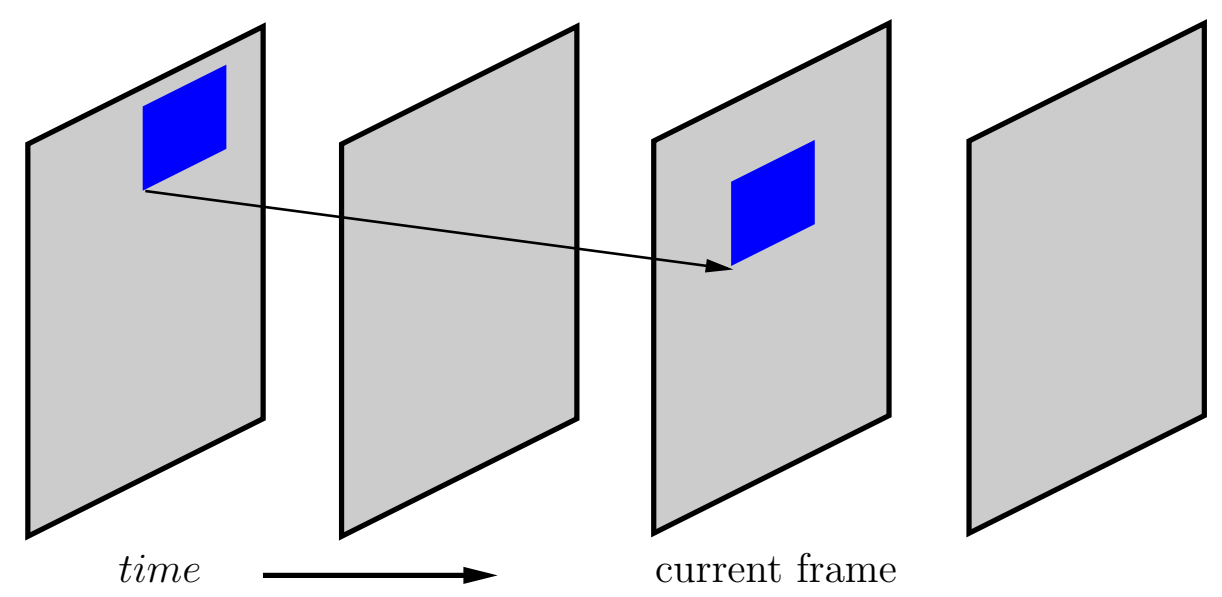

Figure 2.3: Multiframe motion compensation. A reference frame is chosen by an additional picture reference parameter.

rate-constrained motion estimation to trade-off the better prediction signal against the increased bit-rate.

Multiframe block motion compensation in [BG01] makes use of the redundancy that exists across multiple frames in typical video conferencing sequences to achieve additional compression over that obtained by using single frame block motion compensation. The multiframe approach also has an inherent ability to overcome some transmission errors and is thus more robust when compared to the single frame approach. Additional robustness is achieved by randomized frame selection among the multiple previous frames.

Annex U of ITU-T Rec. H.263 [ITU00], entitled "Enhanced Reference Picture Selection Mode," provides multiframe capability for both improved compression efficiency and enhanced resilience to temporal error propagation due to transmission errors. The multiframe concept is also incorporated into the emerging ITU-T Rec. H.264 [ITU02].

\subsubsection{Superimposed Motion Compensation}

Standard block-based motion compensation approximates each block in the current frame by a spatially displaced block chosen from the previous frame. As an extension, long-term memory motion compensation chooses the blocks from several previously decoded frames [WZG99]. The motion-compensated signal is determined by the transmitted motion vector and picture reference parameter.

Now, consider $N$ motion-compensated signals, also called hypotheses. The superimposed prediction signal is the linear superposition of these $N$ hypotheses. Constant scalar coefficients determine the weight of each hypothesis for the predicted block. Only $N$ scalar coefficients are used and each coefficient is applied to all pixel values of the corresponding hypothesis. That is, spatial filtering and OBMC are not employed. Note, that weighted averaging of a set of images is advantageous in the presence of noise as discussed by UNSER and EDEN in [UE90]. 


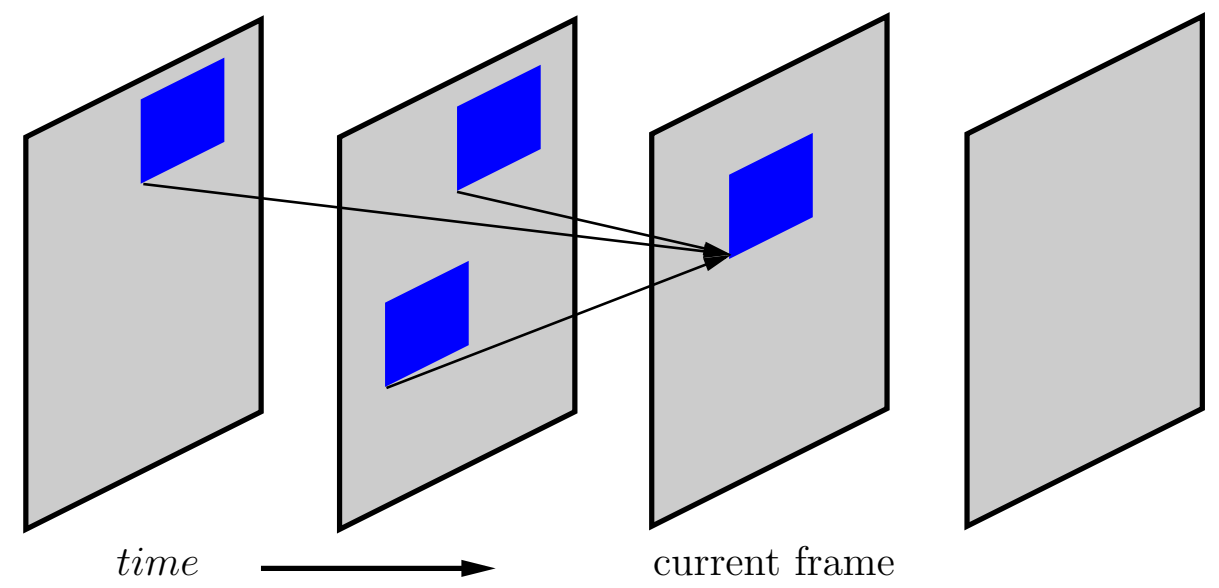

Figure 2.4: Superimposed motion-compensated prediction with three hypotheses. Three blocks of previous decoded frames are linearly combined to form a prediction signal for the current frame.

Fig. 2.4 shows three hypotheses from previous decoded frames which are linearly combined to form the superimposed prediction signal for the current frame. Please note that a hypothesis can be chosen from any reference frame. Therefore, each hypothesis has to be assigned an individual picture reference parameter [Fli97a, FWG98, WFG98, GWS ${ }^{+}$98].

This scheme differs from the concept of B-frame prediction in three significant ways: First, all reference frames are chosen from the past. No reference is made to a subsequent frame, as with B-frames, and hence no extra delay is incurred. Second, hypotheses are not restricted to stem from particular reference frames due to the picture reference parameter. This enables the encoder to find a much more accurate set of prediction signals, at the expense of a minor increase in the number of bits needed to specify them. Third, it is possible to combine more than two motion-compensated signals. As will be shown later, these three properties of superimposed motion compensation improve the coding efficiency of a H.263 codec without incurring the delay that would be caused by using B-pictures.

A special forward prediction mode with two averaged prediction signals is specified in MPEG-2 [ISO96, PMGF96]. This mode is called "Dual Prime Prediction" and can be used for predictive compression of interlaced video. It is utilized in P-pictures where there are no B-pictures between the predicted and the reference pictures. Dual prime is a forward field prediction where a single forward motion vector is estimated for each macroblock of the predicted frame picture. This motion vector points at the reference frame which is the most recent reconstructed frame. Using this vector, each field in the macroblock is associated with a field of the same parity in the reference frame. Two motion vectors pointing at fields of opposite parity are derived from the estimated motion vector by assuming a linear motion trajectory. That is, the motion vectors are scaled according to the temporal distance between the reference and the predicted frames. The two motion-compensated signals are simply averaged to form the prediction.

The design of the superimposed motion-compensated predictor is such that the mean 
square prediction error is minimized while limiting the bit-rate consumed by the motion vectors and picture reference parameters. With variable length coding of the side information, the best choice of hypotheses will depend on the code tables used, while the best code tables depend on the probabilities of choosing certain motion vector/reference parameter combinations. Further, the best choice of hypotheses also depends on the linear coefficients used to weight each hypothesis, while the best coefficients depend on the covariance matrix of the hypotheses.

To solve this design problem, it is useful to interpret superimposed motion-compensated prediction as a vector quantization problem [Ger90, Gra84, GG92]. Entropy Constrained Vector Quantization (ECVQ) [CLG89, SG88], which is an extension of the Generalized Lloyd Algorithm [SG86], is employed to solve the design problem iteratively. For the interpretation of motion-compensated prediction, we argue that a block in the current frame is quantized. The output index of the quantizer is the index of the displacement vector. Each displacement vector is represented by a unique entropy codeword. Further, the codebook used for quantization contains motion-compensated blocks chosen from previous frames. This codebook is adaptive as the reference frames change with the current frame. For superimposed prediction, the codebook contains $N$-tuple of motioncompensated blocks whose components are linearly combined.

Rate-constrained superimposed motion estimation utilizes a Lagrangian cost function. The costs are calculated by adding the mean square prediction error to a rate-term for the motion information, which is weighted by a Lagrange multiplier [Eve63]. The estimator minimizes this cost function on a block basis to determine multiple displacement parameter. This corresponds to the biased nearest neighbor condition familiar from vector quantization with rate constraint. The decoder combines linearly more than one motion-compensated signal which are determined by multiple displacement parameter. In [Fli97a, FWG98], several video sequences are encoded to show that the converged design algorithm just averages multiple hypotheses.

Superimposed motion compensation requires the estimation of multiple motion vectors and picture reference parameters. Best prediction performance is obtained when the $N$ motion vectors and picture reference parameters are jointly estimated. This joint estimation is computationally very demanding. Complexity can be reduced by an iterative algorithm which improves conditional optimal solutions step by step [Fli97a, FWG98]. More details are discussed in Section 2.3.2.

It can be observed that increasing the number of hypotheses also improves the quality of the prediction signal. The gains by superimposed prediction with multiframe motion compensation are larger than with single frame motion compensation. That is, superimposed motion-compensated prediction benefits from multiframe motion compensation such that the PSNR prediction gain is more than additive [Fli97a, FWG98].

It is important to note that an $N$-hypothesis uses $N$ motion vectors and picture reference parameters to form the prediction signal. Applying a product code for these $N$ references will approximately increase the bit-rate for $N$-hypothesis MCP by factor of $N$. This higher rate has to be justified by the improved prediction quality. 
When predicting a target block in the current frame, we have the choice of several different predictors, i.e. 1-hypothesis, 2-hypothesis, . . N-hypothesis predictor. Experiments reveal that each predictor on its own is not the best one in the rate-distortion sense. For the same prediction quality, the 1-hypothesis predictor provides always the lowest bit-rate. On the other hand, improved prediction quality can only be obtained by increasing the number of hypotheses. Therefore, the optimal rate-distortion performance results from selecting the predictor that gives the best rate-distortion performance. Moreover, this selection depends on the block to be predicted [Fli97a, FWG98].

\subsection{Motion Estimation}

Classic motion estimation aims to minimize the energy in the displaced frame difference. But actually, it is a bit allocation problem for both motion vectors and displaced frame difference as they are dependent. RAmChandran, OrTEga and VetTERLi investigate in [ROV94] the problem of bit allocation for dependent quantization. They apply it to the problem of frame type selection like LEE and DiCKInson in [LD94]. But RiBAs-CorberA and Neuhoff in [RCN95] as well as Schuster and Katsaggelos in [SK96] discuss in detail bit allocation between displacement and displaced frame difference. Additional segmentation of the displacement field is investigated in [SK97] and [HW98]. Woods et al. discuss motion vector quantization for video coding [LW95, CW00] as well as multiscale modeling and estimation of motion fields [MKW97]. Rate-constrained motion estimation which utilizes a Lagrangian cost function is discussed by several authors [CAW96, CAW98, CKS96, KLSW97, KCS99].

\subsubsection{Rate-Constrained Motion Estimation}

In the following discussion, we relate the problem of block-based motion-compensated prediction to vector quantization with a rate constraint [Fli97a]. For block-based motioncompensated prediction, each block in the current frame is approximated by a spatially displaced block chosen from a reference picture. Each $a \times a$ block is associated with a vector in the $a^{2}$-dimensional space $\mathcal{R}^{a^{2}}$. A original block is represented by the vectorvalued random variable s. A particular original block is denoted by $s$. The quality of motion-compensated prediction is measured by the average sum square error distortion between original $\mathbf{s}$ and predicted blocks $\hat{\mathbf{s}}$.

$$
D=E\left\{\|\mathbf{s}-\hat{\mathbf{s}}\|_{2}^{2}\right\}
$$

The blocks are coded with a displacement code $\mathbf{b}$. Each displacement codeword $b$ provides a unique rule how to approximate the current block-sample $s$. The average rate of the displacement code is determined by its average length.

$$
R=E\{|\mathbf{b}|\}
$$


Rate-distortion optimal motion-compensated prediction minimizes average prediction distortion subject to a given average displacement rate. This constrained problem can be converted to an unconstrained problem by defining a Lagrangian cost function $J$ with a Lagrange multiplier $\lambda$ [Eve63, CLG89, GG92, Lig95].

$$
J(\lambda)=E\left\{\|\mathbf{s}-\hat{\mathbf{s}}\|_{2}^{2}+\lambda|\mathbf{b}|\right\}
$$

The predictor with the minimum Lagrangian costs is also a rate-distortion optimal predictor.

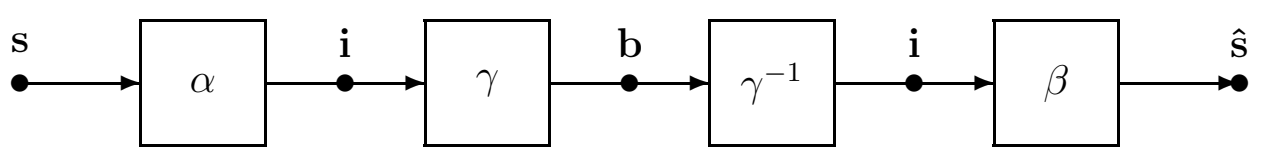

Figure 2.5: Interpreting motion-compensated prediction as vector quantization.

The ECVQ algorithm [CLG89] suggests a vector quantizer model according to Fig. 2.5. This model is interpreted for motion-compensated prediction as follows: Given the original block $\mathbf{s}$, the mapping $\alpha$ estimates the best displacement index $\mathbf{i}$ in the codebook of reference blocks (frame memory). The mapping $\gamma$ assigns a variable length codeword to each displacement index. To be lossless, $\gamma$ has to be invertible and uniquely decodable [CLG89]. For block-based motion-compensated prediction, $\beta$ is a codebook look-up of reference blocks to determine the block $\hat{\mathbf{s}}$ that is used for prediction.

Minimizing the Lagrangian costs (2.3) provides the rate-distortion optimum predictor, that is, the optimum mappings $\alpha$ and $\gamma$. For that, the Lagrangian cost function (2.3) is expressed in terms of the model mappings $\alpha$ and $\gamma$,

$$
J(\alpha, \beta, \gamma, \lambda, \mathbf{s})=E\left\{\|\mathbf{s}-\beta \circ \alpha(\mathbf{s})\|_{2}^{2}+\lambda|\gamma \circ \alpha(\mathbf{s})|\right\}
$$

with the blocks $\hat{\mathbf{s}}=\beta \circ \alpha(\mathbf{s})$ used for prediction and the codewords $\mathbf{b}=\gamma \circ \alpha(\mathbf{s})$. For a given distribution of the original blocks $\mathbf{s}_{c}$ in the training set and constant Lagrange multiplier $\lambda_{c}$, the optimal predictor incorporates the optimal mappings $\alpha$ and $\gamma$ which satisfy

$$
\min _{\{\alpha, \gamma\}} J\left(\alpha, \beta, \gamma, \lambda_{c}, \mathbf{s}_{c}\right)
$$

Given the distribution of the original blocks $\mathbf{s}_{c}$ in the training set as well as the Lagrange multiplier $\lambda_{c}$, an iterative design algorithm for solving (2.5) includes two steps. For motion-compensated prediction, $\beta_{c}$ retrieves the compensated block from the frame memory, which is simply a codebook look-up. The first step determines the optimal displacement index $\mathbf{i}=\alpha(\mathbf{s})$ for the given mapping $\gamma_{c}$.

$$
\begin{aligned}
& \min _{\{\alpha\}} E\left\{\left\|\mathbf{s}_{c}-\beta_{c} \circ \alpha\left(\mathbf{s}_{c}\right)\right\|_{2}^{2}+\lambda_{c}\left|\gamma_{c} \circ \alpha\left(\mathbf{s}_{c}\right)\right|\right\} \\
\Longrightarrow & \alpha(s)=\underset{\{i\}}{\operatorname{argmin}}\left\{\left\|s-\beta_{c}(i)\right\|_{2}^{2}+\lambda_{c}\left|\gamma_{c}(i)\right|\right\}
\end{aligned}
$$

(2.6) is the biased nearest neighbor condition familiar from vector quantization with a rate-constraint. The second step determines the optimal entropy coding $\gamma$ for the given 
motion estimation $\alpha_{c}$. For a given $\alpha_{c}$, the distribution of the displacement indices $\mathbf{i}_{c}$ is constant.

$$
\begin{aligned}
& \min _{\{\gamma\}} E\left\{\left\|\mathbf{s}_{c}-\beta_{c} \circ \alpha_{c}\left(\mathbf{s}_{c}\right)\right\|_{2}^{2}+\lambda_{c}\left|\gamma \circ \alpha_{c}\left(\mathbf{s}_{c}\right)\right|\right\} \\
\Longrightarrow & \min _{\{\gamma\}} E\left\{\left|\gamma\left(\mathbf{i}_{c}\right)\right|\right\}
\end{aligned}
$$

(2.7) postulates the minimum average codeword length of the displacement code, given the displacement indices. This problem can be solved with, e.g., the Huffman algorithm. Finally, given the entropy code $\gamma$, the problem of rate-constrained motion estimation is solved by (2.6).

\subsubsection{Rate-Constrained Estimation of Superimposed Motion}

For rate-constrained estimation of superimposed block motion, the same methodology is employed as outlined for rate-constrained motion estimation. Each block in the current frame is approximated by more than one spatially displaced block chosen from the set of reference pictures. Each $a \times a$ block is associated with a vector in the $a^{2}$-dimensional space $\mathcal{R}^{a^{2}}$. A original block is represented by the vector-valued random variable s. A particular original block is denoted by $s$.

The vector quantizer model according to Fig. 2.5 is interpreted for motion-compensated prediction with $N$ superimposed blocks as follows: Given the original block s, the mapping $\alpha$ estimates again the best displacement index $\mathbf{i}$. The mapping $\gamma$ assigns a variable length codeword to each displacement index. $\gamma$ is invertible and uniquely decodable. For motion-compensated prediction with superimposed blocks, $\beta$ is a codebook look-up of a row-vector $\mathbf{c}=\left(\mathbf{c}_{1}, \mathbf{c}_{2}, \ldots, \mathbf{c}_{N}\right)$ of $N$ motion-compensated blocks. These $N$ motioncompensated blocks are superimposed and determine the block $\hat{\mathbf{s}}$ that is used to predict s. The weighted superposition of $N$ motion-compensated blocks is accomplished with $N$ scalar weights $h_{\mu}, \mu=1,2, \ldots, N$, that sum to one. For simplicity, the vector of scalar weights is denoted by $h$.

$$
\hat{\mathbf{s}}=\mathbf{c} h=\left(\mathbf{c}_{1}, \mathbf{c}_{2}, \ldots, \mathbf{c}_{N}\right)\left(\begin{array}{c}
h_{1} \\
h_{2} \\
\vdots \\
h_{N}
\end{array}\right)
$$

For the scheme of superimposed motion, minimizing the Lagrangian costs (2.3) provides the rate-distortion optimum predictor for superimposed blocks, that is, the optimum mappings $\alpha, \beta$, and $\gamma$. The Lagrangian cost function (2.3) is expressed in terms of the model mappings $\alpha, \beta$, and $\gamma$ according to (2.4). The blocks $\hat{\mathbf{s}}=\beta \circ \alpha(\mathbf{s})$ are used to predict $\mathbf{s}$, and the codewords $\mathbf{b}=\gamma \circ \alpha(\mathbf{s})$ to encode the displacement indices. For a given distribution of the original blocks $\mathbf{s}_{c}$ in the training set and constant Lagrange multiplier $\lambda_{c}$, the optimal predictor incorporates the optimal mappings $\alpha, \beta$, and $\gamma$ which satisfy

$$
\min _{\{\alpha, \beta, \gamma\}} J\left(\alpha, \beta, \gamma, \lambda_{c}, \mathbf{s}_{c}\right) .
$$


Given the distribution of the original blocks $\mathbf{s}_{c}$ in the training set as well as the Lagrange multiplier $\lambda_{c}$, an iterative design algorithm for solving (2.9) includes three steps. For superimposed motion-compensated prediction, $\beta$ is also optimized. The first step determines the optimal displacement indices $\mathbf{i}=\alpha(\mathbf{s})$ for the given mappings $\beta_{c}$ and $\gamma_{c}$.

$$
\begin{aligned}
& \min _{\{\alpha\}} E\left\{\left\|\mathbf{s}_{c}-\beta_{c} \circ \alpha\left(\mathbf{s}_{c}\right)\right\|_{2}^{2}+\lambda_{c}\left|\gamma_{c} \circ \alpha\left(\mathbf{s}_{c}\right)\right|\right\} \\
\Longrightarrow & \alpha(s)=\underset{\{i\}}{\operatorname{argmin}}\left\{\left\|s-\beta_{c}(i)\right\|_{2}^{2}+\lambda_{c}\left|\gamma_{c}(i)\right|\right\}
\end{aligned}
$$

(2.10) is the biased nearest neighbor condition familiar from vector quantization with a rate-constraint. The second step determines the optimal entropy coding $\gamma$ for the given estimation of superimposed motion $\alpha_{c}$. For given $\alpha_{c}$, the distribution of the displacement indices $\mathbf{i}_{c}$ is constant.

$$
\begin{aligned}
& \min _{\{\gamma\}} E\left\{\left\|\mathbf{s}_{c}-\beta_{c} \circ \alpha_{c}\left(\mathbf{s}_{c}\right)\right\|_{2}^{2}+\lambda_{c}\left|\gamma \circ \alpha_{c}\left(\mathbf{s}_{c}\right)\right|\right\} \\
\Longrightarrow & \min _{\{\gamma\}} E\left\{\left|\gamma\left(\mathbf{i}_{c}\right)\right|\right\}
\end{aligned}
$$

(2.11) postulates a minimum average codeword length of the displacement code, given the displacement indices. The third step determines the optimal superposition, i.e., the optimal scalar weights, given the mappings $\alpha_{c}$ and $\gamma_{c}$.

$$
\begin{aligned}
& \min _{\{\beta\}} E\left\{\left\|\mathbf{s}_{c}-\beta \circ \alpha_{c}\left(\mathbf{s}_{c}\right)\right\|_{2}^{2}+\lambda_{c}\left|\gamma_{c} \circ \alpha_{c}\left(\mathbf{s}_{c}\right)\right|\right\} \\
\Longrightarrow & \min _{\left\{h: \mathbf{1}^{T} h=1\right\}} E\left\{\left\|\mathbf{s}_{c}-\mathbf{c}_{c} h\right\|_{2}^{2}\right\}
\end{aligned}
$$

(2.12) is the Wiener problem for the conditional optimal superposition coefficients. The superimposed predictor preserves the expected value of the original block, i.e., $E\{\mathbf{s}\}=$ $E\{\hat{\mathbf{s}}\}$. Consequently, the Wiener problem can be expressed in covariance notation

$$
\min _{\left\{h: \mathbf{1}^{T} h=1\right\}}\left\{C_{\mathbf{s s}}-2 h^{T} C_{\mathbf{c s}}+h^{T} C_{\mathbf{c c}} h\right\}
$$

where $C_{\mathbf{s s}}$ is the scalar variance of the original blocks, $C_{\mathbf{c c}}$ the $N \times N$ covariance matrix of the motion-compensated blocks, and $C_{\mathbf{c s}}$ the $N \times 1$ covariance vector between the motioncompensated blocks and the original blocks. (2.13) is a constrained Wiener problem as the scalar weights $h_{\mu}$ sum to 1 , i.e., $\mathbf{1}^{T} h=1$. A Lagrangian approach leads to the conditional optimal superposition coefficients

$$
h=C_{\mathbf{c c}}^{-1}\left(C_{\mathbf{c s}}-\frac{\mathbf{1}^{T} C_{\mathbf{c c}}^{-1} C_{\mathbf{c s}}-1}{\mathbf{1}^{T} C_{\mathbf{c c}}^{-1} \mathbf{1}} \mathbf{1}\right) .
$$

Experiments with video sequences in [Fli97a, FWG98] reveal that the optimum superposition coefficients are approximately $\frac{1}{N}$ for $N$ superimposed motion-compensated blocks.

Given the entropy code $\gamma$ and the weighted superposition $\beta$, the problem of rateconstrained estimation of superimposed motion is solved by (2.10). The complexity of 
superimposed motion estimation increases exponentially with the number of superimposed blocks $N$. An iterative algorithm, which is inspired by the Iterated Conditional Modes [Bes86], avoids searching the complete space by successively improving $N$ conditional optimal solutions. Convergence to a local optimum is guaranteed, because the algorithm prohibits an increase of the Lagrangian costs.

0: Assuming N superimposed motion-compensated blocks, the Lagrangian cost function

$$
j\left(c_{1}, \ldots, c_{\mu}, \ldots, c_{N}\right)=\left\|s-\sum_{\nu=1}^{N} c_{\nu} h_{\nu}\right\|_{2}^{2}+\lambda\left|\gamma\left(i\left[c_{1}, \ldots, c_{N}\right]\right)\right|
$$

is subject to minimization for each original block $s$. Select the entropy code $\gamma$, predictor coefficients $h$, and the Lagrange multiplier $\lambda$. Initialize the algorithm with $N$ motion-compensated blocks $\left(c_{1}^{(0)}, \ldots, c_{N}^{(0)}\right)$ and set $k:=0$.

1: Select the $\mu$-th block out of $N$; start from the first and end with the $N$-th.

a: Focus on the $\mu$-th block. All others are kept constant. Select a local neighborhood of the block $c_{\mu}^{(k)}$ as the conditional search space for block $c_{\mu}^{(k+1)}$.

b: Minimize the Lagrangian cost function by full search within the conditional search space and determine the new block $c_{\mu}^{(k+1)}$.

$$
\min _{\left\{c_{\mu}^{(k+1)}\right\}} j\left(c_{1}^{(k+1)}, \ldots, c_{\mu-1}^{(k+1)}, c_{\mu}^{(k+1)}, c_{\mu+1}^{(k)}, \ldots, c_{N}^{(k)}\right)
$$

2: As long as the Lagrangian cost function decreases, continue with step 1 and set $k:=k+1$.

Figure 2.6: Hypothesis Selection Algorithm for block-based superimposed motion estimation.

The Hypothesis Selection Algorithm (HSA) in Fig. 2.6 provides a locally optimal solution for (2.10). The HSA is initialized with $N>1$ motion-compensated blocks by splitting the optimal motion-compensated block for $N=1$. The computational complexity of finding a solution for $N=1$ is rather moderate. This optimal motion-compensated block is repeated $N$ times to generate the initial vector of $N$ motion-compensated blocks. For each of the $N$ motion-compensated blocks in each iteration, the HSA performs a full search within a conditional search space. The size of this conditional search space affects both the quality of the local optimum and the computational complexity of the algorithm [Fli97a, FWG98].

\subsubsection{Quantizer Selection at the Residual Encoder}

Hybrid video codecs usually encode the motion-compensated prediction error signal. For that, a uniform scalar quantizer with quantizer step-size $Q$ is utilized. Rate-constrained 
motion estimation raises the question how to select the quantizer step-size dependent on the Lagrange multiplier $\lambda$. A solution to this problem is suggested in [SW98].

Given the Lagrangian cost function $J=D+\lambda R$, total rate $R$ and distortion $D$ are in equilibrium for $d J=d D+\lambda d R=0$. Consequently, $\lambda$ is the negative derivative of the distortion with respect to the total rate.

$$
\lambda=-\frac{d D}{d R}
$$

The rate of the motion-compensated predictor $R_{p}$ and the rate of the residual encoder $R_{r}$ sum up to the total rate $R$. Thus, we can write

$$
\begin{aligned}
d D & =\frac{\partial D}{\partial R_{p}} d R_{p}+\frac{\partial D}{\partial R_{r}} d R_{r} \\
d R & =d R_{p}+d R_{r}
\end{aligned}
$$

and

$$
d J=\left(\frac{\partial D}{\partial R_{p}}+\lambda\right) d R_{p}+\left(\frac{\partial D}{\partial R_{r}}+\lambda\right) d R_{r}=0 .
$$

(2.18) is the condition for the equilibrium and has to hold for any $d R_{p}$ and $d R_{r}$. As a result, the partial derivatives of the distortion are identical and equal to $-\lambda$.

$$
\lambda=-\frac{d D}{d R}=-\frac{\partial D}{\partial R_{p}}=-\frac{\partial D}{\partial R_{r}}
$$

[Gir94] discusses the optimal rate allocation between motion vector rate and prediction error rate and reports the identity of the partial derivatives. Assuming that the total rate is constant and that an infinitesimal bit has to be assigned, the optimum trade-off is achieved when the decrease in distortion caused by this infinitesimal bit is equal for both motion-compensated prediction and residual encoding.

A memoryless Gaussian signal is assumed for the reconstruction error with the distortion-rate function

$$
D\left(R_{p}, R_{r}\right)=\sigma_{\mathbf{e}}^{2}\left(R_{p}\right) 2^{-2 R_{r}}=\sigma_{\mathbf{e}}^{2}\left(R_{p}\right) e^{-R_{r} 2 \ln 2},
$$

where $\sigma_{\mathbf{e}}^{2}\left(R_{p}\right)$ is the variance of the prediction error as a function of the predictor rate $R_{p}$. The partial derivative provides $\lambda=D\left(R_{p}, R_{r}\right) 2 \ln 2$. Note that the factor $2 \ln 2$ is related to the slope of $6 \mathrm{~dB} /$ bit. At high rates, the rate of the predictor $R_{p}$ is negligible compared to the rate of the residual encoder $R_{r}$ and the distortion $D\left(R_{p}, R_{r}\right)$ is dominated by the quantizer of the residual encoder. A high-rate approximation for the uniform scalar quantizer

$$
D\left(R_{p}, R_{r}\right)=\frac{Q^{2}}{12}
$$

relates the quantizer step-size $Q$ to the distortion $D\left(R_{p}, R_{r}\right)$ and $\lambda$.

$$
\lambda=\frac{\ln 2}{6} Q^{2} \approx 0.1 Q^{2}
$$


In practice, this theoretical relationship is modified to

$$
\lambda \approx 0.2 Q^{2}
$$

In [SW98, WG01a], the functional dependency $\lambda=0.85 \cdot Q P^{2}$ is suggested for the ITU-T Rec. H.263, where $Q P$ is the H.263 quantization parameter. The step-size of the H.263 uniform quantizer is determined by multiplying the quantization parameter by 2, i.e. $Q=2 \cdot Q P$. This result motivates the approximation in (2.23).

\subsubsection{Efficient Motion Estimation}

Multiframe motion-compensated prediction and, in particular, superimposed motion-compensated prediction increase the computational complexity of motion estimation. Fast search algorithms can be used to reduce the computational burden without sacrificing performance.

[LS95] presents a fast exhaustive search algorithm for motion estimation. The basic idea is to obtain the best estimate of the motion vectors by successively eliminating the search positions in the search window and thus decreasing the number of matching evaluations that require very intensive computations.

An improved approach is published in [LT97]. This work proposes a fast blockmatching algorithm that uses fast matching error measures besides the conventional mean absolute error or mean square error. An incoming block in the current frame is compared to candidate blocks within the search window using multiple matching criteria. The fast matching error measures are established on the integral projections, having the advantages of being good block features and having simple complexity in measuring matching errors. Most of the candidate blocks can be rejected only by calculating one or more of the fast matching error measures. The time-consuming computations of mean square error or mean absolute error are performed on only a few candidate blocks that first pass all fast matching criteria.

One fast elimination criterion is based on the well-known triangle inequality. A more interesting one is outlined in the following: Let $e_{i}=s_{i}-\hat{s}_{i}$ be the prediction error for pixel $i=1,2, \ldots, L$ and $L$ be the number of pixels in a block. $s_{i}$ are the pixel values of the block in the current frame and $\hat{s}_{i}$ the pixel values of the block used for prediction. For all error values $e_{i}$, the inequality

$$
\sum_{i=1}^{L}\left(e_{i}-\frac{1}{L} \sum_{j=1}^{L} e_{j}\right)^{2} \geq 0
$$

holds and can be simplified to

$$
\sum_{i=1}^{L} e_{i}^{2} \geq \frac{1}{L}\left(\sum_{j=1}^{L} e_{j}\right)^{2}
$$


(2.25) states that the sum-square error is always larger or equal to the normalized squared difference of block pixel sums.

$$
\sum_{i=1}^{L}\left(s_{i}-\hat{s}_{i}\right)^{2} \geq \frac{1}{L}\left(\sum_{j=1}^{L} s_{j}-\sum_{j=1}^{L} \hat{s}_{j}\right)^{2}
$$

These block pixel sums eliminate efficiently search positions with large errors but do not require time-consuming computations. Moreover, the sum for the incoming block in the current frame $\sum_{j} s_{j}$ needs to be calculated only once.

\subsection{Theory of Motion-Compensated Prediction}

Can the future of a sequence be predicted based on its past? If so, how good could this prediction be? These questions are frequently encountered in many applications that utilize prediction schemes [MF98]. Video applications that use predictive coding are no exception and several researchers take the journey to explore motion-compensated prediction. For example, Cuvelier and VANDEndorPe investigate the statistical properties of prediction error images [VCM95], analyze coding of interlaced or progressive video [CV96b], and explore motion-compensated interpolation [CV96a]. LI and GONZALES propose a locally quadratic model of the motion estimation error criterion function and apply it for subpixel interpolation [LG96]. RiBAS-CorBerA and NeuhofF use their analytical model to optimize motion-vector accuracy in block-based video coding [RCN01]. GULERYUZ and ORCHARD investigate rate-distortion based temporal filtering for video compression [GO96] and provide a rate-distortion analysis of DPCM compression of Gaussian autoregressive sequences [GO01]. PANG and TAN examine the role of the optimum loop filter in hybrid coders [PT94]. WEDI considers aliasing and investigates in [Wed99] a time-recursive interpolation filter for motion compensated prediction. He extends his work to an adaptive interpolation filter [Wed02] and suggests a theoretical basis for adaptive interpolation.

GIROD presents in [GM86, Gir87, Gir93b] an approach to characterize motion-compensated prediction. The approach relates the motion-compensated prediction error to the displacement error caused by inaccurate motion compensation. The model utilizes a Gaussian or uniform distributed displacement error to both capture the average accuracy of motion-compensation and evaluate the impact on the motion-compensated prediction error variance. A detailed discussion of fractional-pel motion-compensation and the efficiency of motion-compensated prediction is investigated in [Gir93a].

In [Gir98, Gir00], this theory has been extended to multihypothesis motion-compensated prediction to investigate multiple, linearly combined motion-compensated prediction signals. The paper introduces a statistical model for multiple motion-compensated signals, also called hypotheses. Each hypothesis utilizes the statistical model in [Gir87]. In particular, it assumes statistical independence among the hypotheses. With this approach, the paper continues discussing the optimum Wiener filter for multihypothesis motion-compensated prediction. 
The discussion is based on a high-rate approximation of the rate-distortion performance of a hybrid video codec. The high-rate approximation assumes that the residual encoder encodes the prediction error with very small distortion. That is, any reference frame at time instance $t$ that will be used for prediction suffers no degradation and is identical to the original frame at time instance $t$. With this assumption, the performance of a hybrid video codec can be investigated dependent on the efficiency of motion-compensated prediction.

\subsubsection{Frame Signal Model}

Let $\mathbf{v}=\{\mathbf{v}[l], l \in \Pi\}$ be a scalar random field over a two-dimensional orthogonal grid $\Pi$ with horizontal and vertical spacing of 1 . The vector $l=(x, y)^{T}$ denotes a particular location in the lattice $\Pi$. We call this a space-discrete frame.

A frame is characterized by its autocorrelation function and power spectral density. The scalar space-discrete cross correlation function [LG94] is defined according to

$$
\phi_{\mathbf{a b}}[l]=E\left\{\mathbf{a}\left[l_{0}+l\right] \mathbf{b}^{*}\left[l_{0}\right]\right\}
$$

where $\mathbf{a}$ and $\mathbf{b}$ are complex-valued, jointly wide-sense stationary random fields, $\mathbf{b}^{*}$ is the complex conjugate of $\mathbf{b}$, and $l_{0} \in \Pi$ is an arbitrary location. For wide-sense stationary random fields, the correlation function does not depend on $l_{0}$ but only on the relative two-dimensional shift $l$. The cross spectral density is defined according to

$$
\Phi_{\mathrm{ab}}(\omega)=\mathcal{F}_{*}\left\{\phi_{\mathbf{a b}}[l]\right\}
$$

where $\omega=\left(\omega_{x}, \omega_{y}\right)^{T}$ is the vector valued frequency and $\mathcal{F}_{*}\{\cdot\}$ the $2 \mathrm{D}$ band-limited discrete-space Fourier transform. In particular, the transform is

$$
\left.\left.\left.\left.\Phi_{\mathbf{a b}}(\omega)=\sum_{l \in \Pi} \phi_{\mathbf{a b}}[l] e^{-j \omega^{T} l} \quad \forall \omega \in\right]-\pi, \pi\right] \times\right]-\pi, \pi\right]
$$

and its inverse is

$$
\phi_{\mathbf{a b}}[l]=\frac{1}{4 \pi^{2}} \int_{-\pi}^{\pi} \int_{-\pi}^{\pi} \Phi_{\mathbf{a b}}(\omega) e^{j l^{T} \omega} d \omega \quad \forall \quad l \in \Pi .
$$

It is assumed that an isotropic, exponentially decaying, space-continuous autocorrelation function

$$
\phi_{\mathbf{v v}}(x, y)=\sigma_{\mathbf{v}}^{2} \rho_{\mathbf{v}}^{\sqrt{x^{2}+y^{2}}}
$$

with spatial correlation coefficient $\rho_{\mathbf{v}}$ and overall signal variance $\sigma_{\mathbf{v}}^{2}=1$ characterizes a space-continuous frame $\mathbf{v}$. If we neglect spectral replications due to sampling, this autocorrelation function corresponds to the isotropic spatial power spectral density

$$
\Phi_{\mathbf{v v}}\left(\omega_{x}, \omega_{y}\right)=\frac{2 \pi}{\omega_{0}^{2}}\left(1+\frac{\omega_{x}^{2}+\omega_{y}^{2}}{\omega_{0}^{2}}\right)^{-\frac{3}{2}}
$$

with $\omega_{0}=-\ln \left(\rho_{\mathbf{v}}\right)$. Please refer to Appendix A.4 for details. A spatial correlation coefficient of $\rho_{\mathbf{v}}=0.93$ is typical for video signals. Band-limiting the space-continuous signals to the frequencies $]-\pi, \pi] \times]-\pi, \pi]$ and sampling them at the lattice locations $\Pi$ provides the space-discrete signals. 


\subsubsection{Signal Model for Motion-Compensated Prediction}

For motion-compensated prediction, the motion-compensated signal $\mathbf{c}$ is used to predict the current frame $\mathbf{s}$. The model assumes that both the current frame $\mathbf{s}$ and the motioncompensated signal $\mathbf{c}$ originate from the model frame $\mathbf{v}$. The model frame captures the statistical properties of a frame without motion and residual noise.

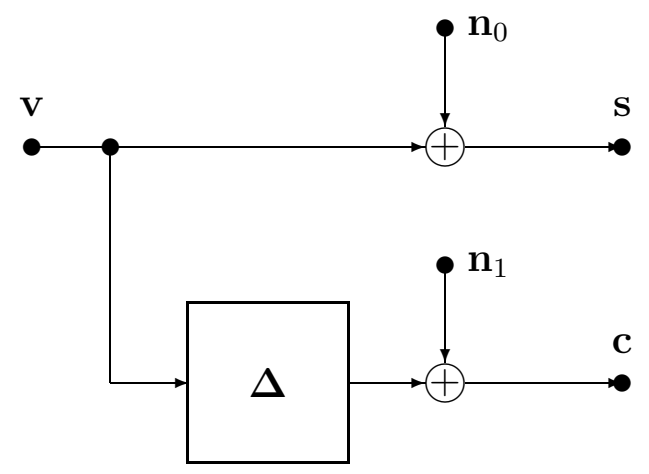

Figure 2.7: Signal model for the current frame $\mathbf{s}$ and the motion-compensated signal $\mathbf{c}$.

Fig. 2.7 depicts the signal model for motion-compensated prediction. Adding statistically independent white Gaussian noise $\mathbf{n}_{0}$ to the model frame $\mathbf{v}$ generates the current frame signal $\mathbf{s}$. Shifting the model frame $\mathbf{v}$ by the statistically independent displacement error $\boldsymbol{\Delta}=\left(\boldsymbol{\Delta}_{\mathbf{x}}, \boldsymbol{\Delta}_{\mathbf{y}}\right)^{T}$ and adding statistically independent white Gaussian noise $\mathbf{n}_{1}$ provides the motion-compensated signal c. For the shift, the ideal reconstruction of the band-limited signal $v[l]$ is shifted by the continuous valued displacement error $\Delta$ and resampled on the original orthogonal grid. The noise signals $\mathbf{n}_{0}$ and $\mathbf{n}_{1}$ are also statistically independent.

The model assumes that the true displacement is known and captures only the displacement error. Obviously, motion-compensated prediction should work best if we compensate the true displacement of the scene exactly for a prediction signal. Less accurate compensation will degrade the performance. To capture the limited accuracy of motion compensation, a vector-valued displacement error $\boldsymbol{\Delta}$ is associated with the motion-compensated signal c. The displacement error reflects the inaccuracy of the displacement vector used for motion compensation and transmission. The displacement vector field can never be completely accurate since it has to be transmitted as side information with a limited bitrate. The model assumes that the 2-D displacement error $\Delta$ is isotropic Gaussian with variance $\sigma_{\Delta}^{2}$

$$
p_{\boldsymbol{\Delta}}(\Delta)=\frac{1}{2 \pi \sigma_{\Delta}^{2}} e^{-\frac{\Delta^{T} \Delta}{2 \sigma_{\Delta}^{2}}} .
$$

With this assumption, the accuracy of motion compensation is now captured by the displacement error variance $\sigma_{\boldsymbol{\Delta}}^{2}$. Further, it is assumed that the displacement error is entirely due to rounding and is uniformly distributed in the interval $\left[-2^{\beta-1}, 2^{\beta-1}\right] \times\left[-2^{\beta-1}, 2^{\beta-1}\right]$, 
where $\beta=0$ for integer-pel accuracy, $\beta=-1$ for half-pel accuracy, $\beta=-2$ for quarter-pel accuracy, etc. Given the displacement inaccuracy $\beta$, the displacement error variance is

$$
\sigma_{\Delta}^{2}=\frac{2^{2 \beta}}{12}
$$

The current frame $\mathbf{s}$ is linearly predicted from the motion-compensated signal $\mathbf{c}$. The prediction error is defined by

$$
\mathbf{e}[l]=\mathbf{s}[l]-f[l] * \mathbf{c}[l]
$$

where $f[l]$ is the impulse response of the 2 -D prediction filter. The asterisk $*$ denotes 2 -D convolution on the original orthogonal grid $\Pi$. The filter is determined according to the minimum mean square error criterion. The normalized power spectral density of the minimum prediction error is determined by

$$
\frac{\Phi_{\mathbf{e e}}(\omega)}{\Phi_{\mathbf{s s}}(\omega)}=1-\frac{1}{1+\alpha_{0}(\omega)} \cdot \frac{P(\omega) P^{*}(\omega)}{1+\alpha_{1}(\omega)}
$$

where $P(\omega)$ is the 2-D continuous-space Fourier transform $\mathcal{F}\left\{p_{\Delta}(\Delta)\right\}$ of the 2-D displacement error PDF $p_{\boldsymbol{\Delta}}(\Delta) . \alpha_{0}(\omega)$ and $\alpha_{1}(\omega)$ are the normalized power spectral densities of the residual noise in the current frame $\mathbf{s}$ and in the motion-compensated signal $\mathbf{c}$, respectively.

$$
\alpha_{\mu}(\omega)=\frac{\Phi_{\mathbf{n}_{\mu} \mathbf{n}_{\mu}}(\omega)}{\Phi_{\mathbf{v v}}(\omega)}
$$

Please note, that the power spectral density of the minimum prediction error is normalized to that of the current frame $\Phi_{\mathbf{s s}}(\omega)$, whereas the power spectral density of the residual noise is normalized to that of the model frame $\Phi_{\mathbf{v v}}(\omega)$.

\subsubsection{Signal Model for Multihypothesis Prediction}

For multihypothesis motion-compensated prediction, $N$ motion-compensated signals $\mathbf{c}_{\mu}$ with $\mu=1,2, \ldots, N$ are used to predict the current frame $\mathbf{s}$. The model assumes that both the current frame $\mathbf{s}$ and $N$ motion-compensated signals $\mathbf{c}_{\mu}$ originate from the model frame $\mathbf{v}$. The model frame captures the statistical properties of a frame without motion and residual noise.

Fig. 2.8 depicts the signal model for multihypothesis motion-compensated prediction. Adding statistically independent white Gaussian noise $\mathbf{n}_{0}$ to the model frame $\mathbf{v}$ generates the current frame signal $\mathbf{s}$. Shifting the model frame $\mathbf{v}$ by the statistically independent displacement error $\boldsymbol{\Delta}_{\mu}$ and adding statistically independent white Gaussian noise $\mathbf{n}_{\mu}$ provides the $\mu$-th motion-compensated signal $\mathbf{c}_{\mu}$. For the shift, the ideal reconstruction of the band-limited signal $v[l]$ is shifted by the continuous valued displacement error $\Delta_{\mu}$ and re-sampled on the original orthogonal grid. The noise signals $\mathbf{n}_{\mu}$ and $\mathbf{n}_{\nu}$ with $\mu, \nu=0,1, \ldots, N$ are mutually statistically independent for $\mu \neq \nu$.

The model assumes that $N$ true displacements exist and utilizes $N$ displacement errors to capture the limited accuracy of the $N$ motion-compensated signals. For that, a 


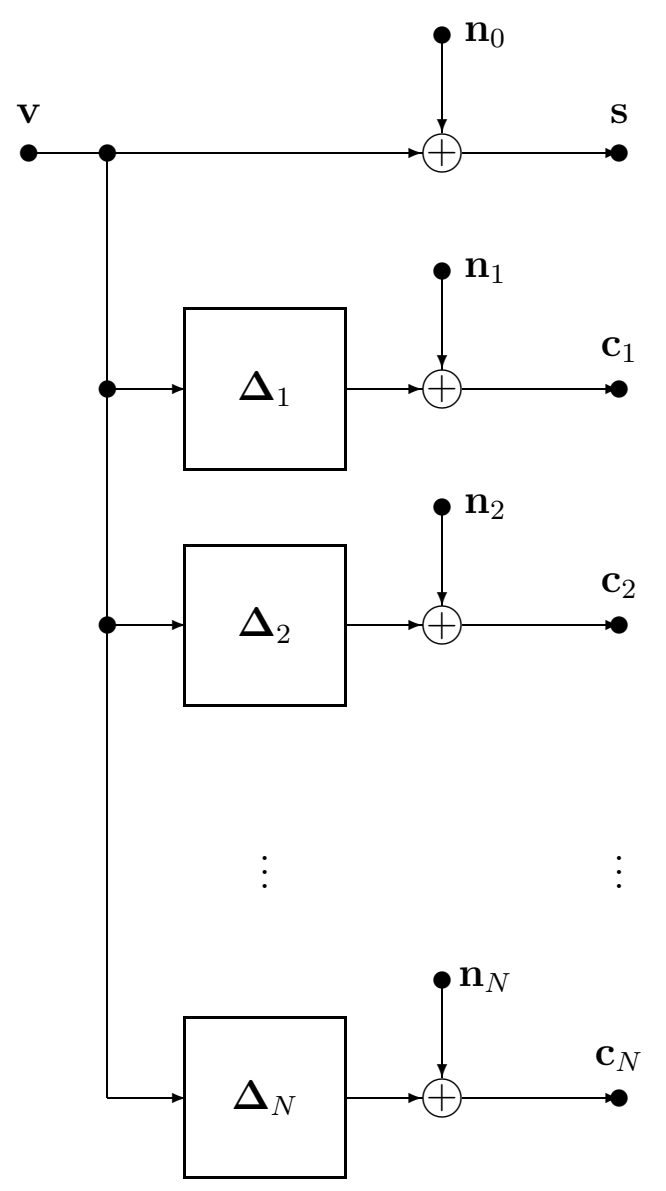

Figure 2.8: Signal model for the current frame $\mathbf{s}$ and $N$ motion-compensated prediction signals (hypotheses) $\mathbf{c}_{\nu}$.

vector-valued displacement error $\boldsymbol{\Delta}_{\mu}$ with $\mu=1,2, \ldots, N$ is associated with the $\mu$-th motion-compensated signal $\mathbf{c}_{\mu}$. The $\mu$-th displacement error reflects the inaccuracy of the $\mu$-th displacement vector used for multihypothesis motion compensation. The model assumes that all displacement errors $\boldsymbol{\Delta}_{\mu}$ with $\mu=1,2, \ldots, N$ are isotropic Gaussian with identical variance $\sigma_{\boldsymbol{\Delta}}^{2}$ according to (2.33). The displacement errors $\boldsymbol{\Delta}_{\mu}$ and $\boldsymbol{\Delta}_{\nu}$ with $\mu, \nu=1,2, \ldots, N$ are mutually statistically independent for $\mu \neq \nu$.

The current frame $\mathbf{s}$ is linearly predicted from the vector of motion-compensated signals $\mathbf{c}=\left(\mathbf{c}_{1}, \mathbf{c}_{2}, \ldots, \mathbf{c}_{N}\right)^{T}$. The scalar prediction error is defined by

$$
\mathbf{e}[l]=\mathbf{s}[l]-f[l] * \mathbf{c}[l]
$$

with the row-vector of impulse responses $f[l]=\left(f_{1}[l], f_{2}[l], \ldots, f_{N}[l]\right)$ of the 2 -D prediction filter. The asterisk $*$ denotes $2-\mathrm{D}$ convolution on the original orthogonal grid $\Pi$ according to

$$
f[l] * \mathbf{c}[l]=\sum_{l_{0} \in \Pi} f\left[l_{0}\right] \mathbf{c}\left[l-l_{0}\right] .
$$


The filter is determined according to the minimum mean square error criterion. The normalized power spectral density of the minimum prediction error is determined by

$$
\frac{\Phi_{\mathbf{e e}}}{\Phi_{\mathrm{ss}}}=1-\frac{1}{1+\alpha_{0}}\left(\begin{array}{c}
P_{1} \\
P_{2} \\
\vdots \\
P_{N}
\end{array}\right)^{H}\left(\begin{array}{cccc}
1+\alpha_{1} & P_{1} P_{2}^{*} & \cdots & P_{1} P_{N}^{*} \\
P_{2} P_{1}^{*} & 1+\alpha_{2} & \cdots & P_{2} P_{N}^{*} \\
\vdots & \vdots & \ddots & \vdots \\
P_{N} P_{1}^{*} & P_{N} P_{2}^{*} & \cdots & 1+\alpha_{N}
\end{array}\right)^{-1}\left(\begin{array}{c}
P_{1} \\
P_{2} \\
\vdots \\
P_{N}
\end{array}\right) .
$$

For simplicity, the argument $\omega$ is omitted. $P_{\mu}(\omega)$ is the 2-D continuous-space Fourier transform $\mathcal{F}\left\{p_{\boldsymbol{\Delta}_{\mu}}(\Delta)\right\}$ of the $\mu$-th 2-D displacement error PDF $p_{\boldsymbol{\Delta}_{\mu}}(\Delta) . \alpha_{0}(\omega)$ and $\alpha_{\mu}(\omega)$ with $\mu=1,2, \ldots, N$ are the normalized power spectral densities of the residual noise in the current frame $\mathbf{s}$ and in the motion-compensated signals $\mathbf{c}_{\mu}$, respectively. The normalized power spectral density of the residual noise is defined according to (2.37). Please note, that the power spectral density of the minimum prediction error is normalized to that of the current frame $\Phi_{\mathbf{s s}}(\omega)$, whereas the power spectral density of the residual noise is normalized to that of the model frame $\Phi_{\mathbf{v v}}(\omega)$.

\subsubsection{Performance Measures}

With high-rate assumptions, the motion-compensated prediction error e is sufficient for performance evaluation. As the spatial correlation of the prediction error is only weak, the potential for redundancy reduction in the residual encoder is relatively small [Gir00]. This suggests that the prediction error variance

$$
\sigma_{\mathbf{e}}^{2}=\frac{1}{4 \pi^{2}} \int_{-\pi}^{\pi} \int_{-\pi}^{\pi} \Phi_{\mathbf{e e}}(\omega) d \omega
$$

is a useful measure that is related to the minimum achievable transmission bit-rate for a given signal-to-noise ratio [JN84]. The minimization of the prediction error variance (2.41) is widely used to obtain the displacement vector and control the coding mode in practical systems. A more refined measure is the rate difference [Ber71]

$$
\Delta R=\frac{1}{8 \pi^{2}} \int_{-\pi}^{\pi} \int_{-\pi}^{\pi} \log _{2}\left(\frac{\Phi_{\mathbf{e e}}(\omega)}{\Phi_{\mathbf{s s}}(\omega)}\right) d \omega .
$$

In (2.42), $\Phi_{\mathbf{e e}}(\omega)$ and $\Phi_{\mathbf{s s}}(\omega)$ are the power spectral densities of the prediction error $\mathbf{e}$ and the current frame s, respectively. Unlike (2.41), the rate difference (2.42) takes the spatial correlation of the prediction error $\mathbf{e}$ and the original signal $\mathbf{s}$ into account. It represents the maximum bit-rate reduction (in bits/sample) possible by optimum encoding of the prediction error e, compared to optimum intra-frame encoding of the signal s, for Gaussian wide-sense stationary signals for the same mean square reconstruction error [JN84]. A negative $\Delta R$ corresponds to a reduced bit-rate compared to optimum intra-frame coding, while a positive $\Delta R$ is a bit-rate increase due to motion-compensation, as it can occur for 
inaccurate motion-compensation. The maximum bit-rate reduction can be fully realized at high bit-rates, while for low bit-rates the actual gain is smaller. The rate required for transmitting the displacement estimate is neglected. The optimum balance between rates for the prediction error signal and displacement vectors strongly depends on the total bit-rate. For high rates, it is justified to neglect the rate for the displacement vectors [Gir00].

\subsubsection{Conclusions}

Based on the simplifying assumptions, several important conclusions can be drawn from the theory. Doubling the accuracy of motion compensation, such as going from integer-pel to 1/2-pel accuracy, can reduce the bit-rate by up to 1 bit per sample independent of $N$ for the noise-free case. An optimum combination of $N$ hypotheses always lowers the bitrate for increasing $N$. If each hypotheses is equally good in terms of displacement error PDF, doubling $N$ can yield a gain of 0.5 bits per sample if there is no residual noise. If realistic residual noise levels are taken into account, the gains possible by doubling the number of hypotheses, $N$, decreases with increasing $N$. Diminishing returns and, ultimately, saturation is observed. If the residual noise power increases, doubling and ultimately quadrupling the number of hypotheses $N$ becomes more efficient than doubling the accuracy of motion compensation. The critical accuracy beyond which the gain due to more accurate motion compensation is small moves to larger displacement error variances with increasing noise and increasing number of hypotheses $N$. Hence, sub-pel accurate motion compensation becomes less important with multihypothesis motion-compensated prediction. Spatial filtering of the motion-compensated candidate signals becomes less important if more hypotheses are combined [Gir00].

\subsection{Three-Dimensional Subband Coding of Video}

Hybrid video coding schemes utilize predictive coding with motion-compensated prediction for efficient compression. Such compression schemes require sequential processing of video signals which makes it difficult to achieve efficient embedded representations. A multiresolution signal decomposition of the video signal seems to be promising to achieve efficient embedded representations. MALlAT [Mal89] discusses the wavelet representation as a suitable tool for multiresolution signal decomposition. Moreover, wavelets are also suitable for coding applications [VK95, TM01]. For example, SHAPIRO proposes zerotrees of wavelet coefficients for embedded image coding [Sha93]. UsEvITCH derives optimal bit allocations for biorthogonal wavelet coders which result in minimum reconstruction error [Use96]. TAUBMAN proposes a new image compression algorithm based on independent Embedded Block Coding with Optimized Truncation of the embedded bit-stream (EBCOT) [Tau00].

Practical wavelet coding schemes are characterized by the construction of the wavelets. Daubechies and Sweldens factor wavelet transforms into lifting steps [DS98, Swe95, 
Swe98]. This construction scheme permits wavelet transforms that map integers to integers [CDSY98], a desirable property for any practical coding scheme. Moreover, the lifting scheme permits also adaptive wavelet transforms [CDSB97, CBN98]. Adaptive schemes like motion compensation can be incorporated into the lifting scheme.

\subsubsection{Motion Compensation and Subband Coding}

For hybrid video coding, there are attempts to keep the efficient predictive architecture with motion compensation and to use wavelet-based techniques for coding the displaced frame difference, e.g. [MC99]. These are spatial subband coding techniques and do not provide three-dimensional subbands. Early attempts of three-dimensional subband coding did not employ motion compensation. KNAUER applied in [Kna76] the Hadamard transform for real-time television compression by considering the sequence of frames as a volume. Further research is published by Vetterli et al. [KV88], Biemond et al. [BLB92], and JAYANT et al. [PJF95]. Interestingly, ZhANG et al. investigate memoryconstrained 3D wavelet transforms without boundary effects and use the lifting structure [XLXZ00, XXLZ02]. BARLAUD et al. suggest a 3D scan based wavelet transform with reduced memory requirements [PAB01]. 3D wavelet coding with arbitrary regions of support is discussed by Minami et al. [MXWM01].

Three-dimensional coding with motion compensation has first been suggested by KRONANDER [Kro89, Kro90]. AKIYAMA et al. use global motion compensation and threedimensional transform coding [ATT90]. Global motion compensation with 3D wavelet coding is also investigated by CHOU et al. in [WXCM99]. ZHANG and ZAFAR present a motion-compensated wavelet transform coder for color video compression [ZZ92]. OHM starts his investigation of 3D subband video coding [Ohm94b, OR97] with integer-pel accurate motion compensation and first order quadrature mirror filter in the temporal domain [Ohm92, Ohm93]. Woods et al. discuss a scheme for object-based spatio-temporal subband coding [HW97]. They optimize the trade-off of the rate between motion vectors and 3-D subbands [CW99] and consider digital cinema applications [CW02]. A bit allocation scheme for subband compression of HDTV is published in [WN92]. For video communication over wireless channels, CHOU and CHEN propose a perceptually optimized 3-D subband codec [CC96].

The multiresolution signal decomposition of the video signal permits temporal, spatial, and rate-distortion scalability. This important feature is investigated by many researchers. Uz et al. suggest a scheme for interpolative multiresolution coding of digital HDTV [UVL90, UVL91]. In addition to the multiresolution representation of the motion-compensated three-dimensional signal, researchers investigate also the multiresolution representation of motion, like OHм [Ohm94a] and ZhANG et al. [ZZJ93]. TAubman and ZAKHOR propose a common framework for rate and distortion based scaling of highly scalable compressed video [TZ94c, TZ94a, TZ94b, Tau94, TZ96]. PEARLMAn et al. introduce in [KP97, KXP00] an embedded wavelet video codec using three-dimensional set partitioning in hierarchical trees [SP96]. WooDs et al. discuss rate-constrained multiresolution transmission of video [NBWL95], and present a resolution and frame-rate scalable 
subband video coder [WL01]. RANGANATH et al. outline a highly scalable wavelet-based codec for very low bit-rate environments and introduces tri-zerotrees [TRK98]. PESQUETPopescu et al. suggest a method for context modeling in the spatio-temporal trees of wavelet coefficients [FPP00] and propose the strategy of fully scalable zerotree coding [BBFPP01]. ZHANG et al. investigate three-dimensional embedded block coding with optimized truncation [XXLZ01].

\subsubsection{Motion-Compensated Lifted Wavelets}

The previously mentioned lifting scheme permits adaptive wavelet transforms. For the temporal subband decomposition, Pesquet-Popescu and Bottreau improve the compression efficiency with lifting schemes that use motion compensation [PPB01]. ZHANG et al. also incorporate motion compensation into the lifting scheme [LLL $\left.{ }^{+} 01\right]$ and use this method for 3D wavelet compression of concentric mosaics [LWLZ02]. SECKER and TAUBMAN investigate lifting schemes with block motion compensation [ST01] and deformable mesh motion compensation [ST02]. OHM reviews the recent progress and discusses novel aspects with respect to irregular sampled signals, shift variance of wavelet transforms and non-dyadic wavelet processing [Ohm02]. PATEux et al. investigate several motion-compensated lifting implementations and compare to standardized hybrid codecs [VGP02]. BARLAUd et al. extend their 3D scan-based wavelet transform codec and use the motion-compensated lifting scheme [PAB02]. 


\section{Motion-Compensated Prediction with Complementary Hypotheses}

\subsection{Introduction}

As discussed in Section 2.4, the theoretical investigation in [Gir00] shows that a linear combination of multiple motion-compensated signals can improve the performance of motion-compensated prediction for video coding. This chapter extends that work by introducing the concept of complementary hypotheses [FG01c, FWG02].

To motivate this concept, let us consider pairs of motion-compensated signals. The two signals are simply averaged to form the prediction signal. We ask the question what kind of pairs are necessary to achieve the best prediction performance of superimposed motion compensation. If a pair consists of two identical hypotheses, the superimposed prediction signal is identical to either one of the hypotheses and we expect no improvement over motion-compensated prediction with just one hypothesis. But, in general, there will be pairs of hypotheses that outperform motion-compensated prediction with single hypotheses. Our approach is to model the dependency among the two signals by a correlation coefficient and investigate its impact on the performance of superimposed motion compensation.

Our assumption that there will be $N$-tuples of hypotheses that outperform motioncompensated prediction with single hypotheses is supported by experimental results. As discussed in Section 2.3.2, the work in [Fli97a, FWG98] demonstrates experimentally that such efficient $N$-tuples exist. The work proposes an iterative algorithm for block-based rate-constrained superimposed motion estimation. The algorithm improves conditionally optimal solutions and provides a local optimum for the joint estimation problem. The results demonstrate that joint estimation of hypotheses is important for superimposed motion-compensated prediction.

The outline of this chapter is as follows: Section 3.2 extends the known model of multihypothesis motion-compensated prediction. Correlated displacement error for superimposed prediction are discussed and the concept of motion compensation with complementary hypotheses is introduced. Further, we discuss the gradient of the prediction error variance for superimposed motion-compensated signals and the impact of a particular frame signal model. Section 3.3 analyzes "noisy" hypotheses, investigates both averaging and Wiener filtering, and provides performance results for jointly estimated hypotheses. Section 3.4 explores hypothesis switching as a method to select efficient hypotheses for superimposed motion-compensated prediction. First, the signal model for forward-adaptive 
hypothesis switching is introduced. Second, the problem is approached by minimizing the radial displacement error. Third, a property of the assumed PDF allows the definition of an equivalent predictor. And finally, forward-adaptive hypothesis switching is combined with superimposed motion-compensated prediction. Section 3.5 discusses image sequence coding where individual images are predicted with a varying number of hypotheses. The impact of superimposed motion estimation on the overall coding efficiency is investigated.

\subsection{Extended Model for Superimposed Motion-Com- pensated Prediction}

\subsubsection{Superimposed Prediction and Correlated Displacement Error}

We extend the model for multihypothesis motion-compensated prediction as discussed in Section 2.4 such that correlated displacement errors can be investigated. Let $\mathbf{s}[l]$ and $\mathbf{c}_{\mu}[l]$ be scalar two-dimensional signals sampled on an orthogonal grid with horizontal and vertical spacing of 1 . The vector $l=(x, y)^{T}$ denotes the location of the sample. For the problem of superimposed motion compensation, we interpret $\mathbf{c}_{\mu}$ as the $\mu$-th of $N$ motioncompensated signals available for prediction, and $\mathbf{s}$ as the current frame to be predicted. We also call $\mathbf{c}_{\mu}$ the $\mu$-th hypothesis.

Motion-compensated prediction should work best if we compensate the true displacement of the scene exactly for a prediction signal. Less accurate compensation will degrade the performance. To capture the limited accuracy of motion compensation, we associate a vector-valued displacement error $\boldsymbol{\Delta}_{\mu}$ with the $\mu$-th hypothesis $\mathbf{c}_{\mu}$. The displacement error reflects the inaccuracy of the displacement vector used for motion compensation and transmission. The displacement vector field can never be completely accurate since it has to be transmitted as side information with a limited bit-rate. For simplicity, we assume that all hypotheses are shifted versions of the current frame signal s. The shift is determined by the vector-valued displacement error $\boldsymbol{\Delta}_{\mu}$ of the $\mu$-th hypotheses. For that, the ideal reconstruction of the band-limited signal $\mathbf{s}[l]$ is shifted by the continuous valued displacement error and re-sampled on the original orthogonal grid. For now, the translatory displacement model as depicted in Fig. 2.8 omits "noisy" signal components.

A superimposed motion-compensated predictor forms a prediction signal by averaging $N$ hypotheses $\mathbf{c}_{\mu}[l]$ in order to predict the current frame signal $\mathbf{s}[l]$. The prediction error for each pel at location $l$ is the difference between the current frame signal and $N$ averaged hypotheses

$$
\mathbf{e}[l]=\mathbf{s}[l]-\frac{1}{N} \sum_{\mu=1}^{N} \mathbf{c}_{\mu}[l] .
$$

Assume that $\mathbf{s}$ and $\mathbf{c}_{\mu}$ are generated by a jointly wide-sense stationary random process with the real-valued scalar two-dimensional power spectral density $\Phi_{\mathbf{s s}}(\omega)$ as well as the 
cross spectral densities $\Phi_{\mathbf{c}_{\mu} \mathbf{s}}(\omega)$ and $\Phi_{\mathbf{c}_{\mu} \mathbf{c}_{\nu}}(\omega)$. Power spectra and cross spectra are defined according to $(2.28)$ where $\omega=\left(\omega_{x}, \omega_{y}\right)^{T}$ is the vector valued frequency.

The power spectral density of the prediction error in (3.1) is given by the power spectrum of the current frame and the cross spectra of the hypotheses

$$
\Phi_{\mathbf{e e}}(\omega)=\Phi_{\mathbf{s s}}(\omega)-\frac{2}{N} \sum_{\mu=1}^{N} \Re\left\{\Phi_{\mathbf{c}_{\mu} \mathbf{s}}(\omega)\right\}+\frac{1}{N^{2}} \sum_{\mu=1}^{N} \sum_{\nu=1}^{N} \Phi_{\mathbf{c}_{\mu} \mathbf{c}_{\nu}}(\omega)
$$

where $\Re\{\cdot\}$ denotes the real component of the, in general, complex valued cross spectral densities $\Phi_{\mathbf{c}_{\mu} \mathbf{s}}(\omega)$. We adopt the expressions for the cross spectra from [Gir00], where the displacement errors $\boldsymbol{\Delta}_{\mu}$ are interpreted as random variables which are statistically independent from $\mathbf{s}$ :

$$
\begin{aligned}
\Phi_{\mathbf{c}_{\mu} \mathbf{s}}(\omega) & =\Phi_{\mathbf{S s}}(\omega) E\left\{e^{-j \omega^{T} \boldsymbol{\Delta}_{\mu}}\right\} \\
\Phi_{\mathbf{c}_{\mu} \mathbf{c}_{\nu}}(\omega) & =\Phi_{\mathbf{S s}}(\omega) E\left\{e^{-j \omega^{T}\left(\boldsymbol{\Delta}_{\mu}-\boldsymbol{\Delta}_{\nu}\right)}\right\}
\end{aligned}
$$

Like in [Gir00], we assume a power spectrum $\Phi_{\mathrm{ss}}$ that corresponds to an exponentially decaying isotropic autocorrelation function with a correlation coefficient $\rho_{\mathbf{s}}$.

For the $\mu$-th displacement error $\boldsymbol{\Delta}_{\mu}$, a 2-D stationary normal distribution with variance $\sigma_{\boldsymbol{\Delta}}^{2}$ and zero mean is assumed where the $x$ - and $y$-components are statistically independent. The displacement error variance is the same for all $N$ hypotheses. This is reasonable because all hypotheses are compensated with the same accuracy. Further, the pairs $\left(\boldsymbol{\Delta}_{\mu}, \boldsymbol{\Delta}_{\nu}\right)$ are assumed to be jointly Gaussian random variables. The predictor design in [FWG98] showed that there is no preference among the $N$ hypotheses. Consequently, the correlation coefficient $\rho_{\boldsymbol{\Delta}}$ between two displacement error components $\boldsymbol{\Delta}_{\mathbf{x} \mu}$ and $\boldsymbol{\Delta}_{\mathbf{x} \nu}$ is the same for all pairs of hypotheses. The above assumptions are summarized by the covariance matrix of a displacement error component.

$$
C_{\boldsymbol{\Delta}_{\mathrm{x}} \boldsymbol{\Delta}_{\mathrm{x}}}=\sigma_{\boldsymbol{\Delta}}^{2}\left(\begin{array}{cccc}
1 & \rho_{\boldsymbol{\Delta}} & \cdots & \rho_{\boldsymbol{\Delta}} \\
\rho_{\boldsymbol{\Delta}} & 1 & \cdots & \rho_{\boldsymbol{\Delta}} \\
\vdots & \vdots & \ddots & \vdots \\
\rho_{\boldsymbol{\Delta}} & \rho_{\boldsymbol{\Delta}} & \cdots & 1
\end{array}\right)
$$

Since the covariance matrix is nonnegative definite [Pap91], the correlation coefficient $\rho_{\boldsymbol{\Delta}}$ in (3.5) has the limited range

$$
\frac{1}{1-N} \leq \rho_{\Delta} \leq 1 \quad \text { for } \quad N=2,3,4, \ldots
$$

which is dependent on the number of hypotheses $N$. More details on this result can be found in Appendix A.1. In contrast to [Gir00], we do not assume that the displacement errors $\boldsymbol{\Delta}_{\mu}$ and $\boldsymbol{\Delta}_{\nu}$ are mutually independent for $\mu \neq \nu$.

These assumptions allow us to express the expected values in (3.3) and (3.4) in terms of the 2-D Fourier transform $P$ of the continuous 2-D probability density function of the 
displacement error $\boldsymbol{\Delta}_{\mu}$.

$$
\begin{aligned}
E\left\{e^{-j \omega^{T} \boldsymbol{\Delta}_{\mu}}\right\} & =\int_{\mathcal{R}^{2}} p_{\boldsymbol{\Delta}_{\mu}}(\Delta) e^{-j \omega^{T} \Delta} d \Delta \\
& =P\left(\omega, \sigma_{\Delta}^{2}\right) \\
& =e^{-\frac{1}{2} \omega^{T} \omega \sigma_{\Delta}^{2}}
\end{aligned}
$$

The expected value in (3.4) contains differences of jointly Gaussian random variables. The difference of two jointly Gaussian random variables is also Gaussian. As the two random variables have equal variance $\sigma_{\boldsymbol{\Delta}}^{2}$, the variance of the difference signal is given by $\sigma^{2}=2 \sigma_{\boldsymbol{\Delta}}^{2}\left(1-\rho_{\boldsymbol{\Delta}}\right)$. Therefore, we obtain for the expected value in (3.4)

$$
E\left\{e^{-j \omega^{T}\left(\boldsymbol{\Delta}_{\mu}-\boldsymbol{\Delta}_{\nu}\right)}\right\}=P\left(\omega, 2 \sigma_{\boldsymbol{\Delta}}^{2}\left(1-\rho_{\boldsymbol{\Delta}}\right)\right) \quad \text { for } \quad \mu \neq \nu .
$$

For $\mu=\nu$, the expected value in (3.4) is equal to one. With that, we obtain for the power spectrum of the prediction error in (3.2):

$$
\frac{\Phi_{\mathrm{ee}}(\omega)}{\Phi_{\mathbf{s s}}(\omega)}=\frac{N+1}{N}-2 P\left(\omega, \sigma_{\Delta}^{2}\right)+\frac{N-1}{N} P\left(\omega, 2 \sigma_{\boldsymbol{\Delta}}^{2}\left(1-\rho_{\boldsymbol{\Delta}}\right)\right)
$$

Setting $\rho_{\Delta}=0$ provides a result which is presented in [Gir00], equation (23), with negligible noise $\alpha_{\mu}=0$, averaging filter $F$, and identical characteristic functions $P_{\mu}=P$.

\subsubsection{Complementary Hypotheses}

The previous section shows that the displacement error correlation coefficient influences the performance of superimposed motion compensation. An ideal superimposed motion estimator will select sets of hypotheses that optimize the performance of superimposed motion compensation. In the following, we focus on the relationship between the prediction error variance

$$
\sigma_{\mathbf{e}}^{2}=\frac{1}{4 \pi^{2}} \int_{-\pi}^{\pi} \int_{-\pi}^{\pi} \Phi_{\mathbf{e e}}(\omega) d \omega
$$

and the displacement error correlation coefficient. The prediction error variance is a useful measure because it is related to the minimum achievable transmission bit-rate [Gir00].

Fig. 3.1 depicts the dependency of the normalized prediction error variance on the displacement error correlation coefficient $\rho_{\boldsymbol{\Delta}}$ within the range (3.6). The dependency is plotted for $N=2,4,8$, and $\infty$ for integer-pel accurate motion compensation $\left(\sigma_{\Delta}^{2}=1 / 12\right)$. The correlation coefficient of the frame signal $\rho_{\mathbf{s}}=0.93$ [Gir00]. Reference is the prediction error variance of the single hypothesis predictor $\sigma_{\mathbf{e}, 1}^{2}$. We observe that a decreasing correlation coefficient lowers the prediction error variance. (3.9) implies that this observation holds for any displacement error variance. Fig. 3.1 shows also that identical displacement errors $\left(\rho_{\boldsymbol{\Delta}}=1\right)$ do not reduce the prediction error variance compared to single hypothesis motion compensation. This is reasonable when we consider identical hypotheses. They do 


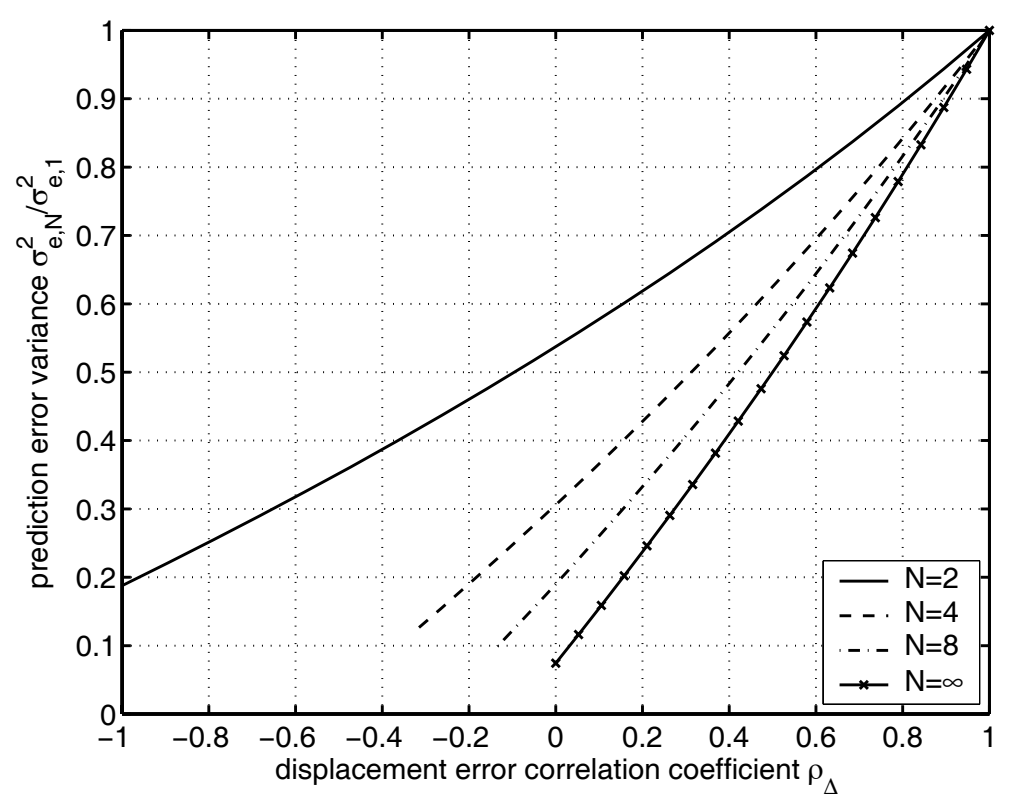

Figure 3.1: Normalized prediction error variance for superimposed MCP over the displacement error correlation coefficient $\rho_{\boldsymbol{\Delta}}$. Reference is the single hypothesis predictor. The hypotheses are averaged and no residual noise is assumed. The variance of the displacement error is set to $\sigma_{\Delta}^{2}=1 / 12$.

not improve superimposed motion-compensation because they have identical displacement errors.

To determine the performance bound, we assume the existence of all $N$-tuple of hypotheses that obey (3.6) and that an ideal superimposed motion estimator is able to determine any desired $N$-tuple. Assuming a mean square error measure, the optimal ideal superimposed motion estimator minimizes the summed squared error

$$
\min \frac{1}{|\mathcal{L}|} \sum_{l \in \mathcal{L}} e^{2}[l]
$$

and the expected value

$$
\min E\left\{\frac{1}{|\mathcal{L}|} \sum_{l \in \mathcal{L}} \mathbf{e}^{2}[l]\right\},
$$

where $\mathbf{e}[l]$ denotes the prediction error at pixel location $l$. In addition, we assume a stationary error signal such that

$$
E\left\{\mathbf{e}^{2}[l]\right\}=\sigma_{\mathbf{e}}^{2}[l]=\sigma_{\mathbf{e}}^{2} \quad \forall l .
$$

Consequently, this optimal ideal estimator minimizes the prediction error variance.

$$
\min \sigma_{\mathbf{e}}^{2}
$$

Further, $\sigma_{\mathbf{e}}^{2}$ increases monotonically for increasing $\rho_{\boldsymbol{\Delta}}$. This is a property of (3.9) which is also depicted in Fig. 3.1. The minimum of the prediction error variance is achieved at 
the lower bound of $\rho_{\Delta}$.

$$
\min _{\frac{1}{1-N} \leq \rho_{\Delta} \leq 1} \rho_{\Delta}
$$

That is, an optimal ideal superimposed motion estimator minimizes the prediction error variance by minimizing the displacement error correlation coefficient. Its minimum is given by the lower bound of the range (3.6).

$$
\rho_{\Delta}=\frac{1}{1-N} \quad \text { for } \quad N=2,3,4, \ldots
$$

This insight implies an interesting result for the case $N=2$ : Pairs of hypotheses that operate on the performance bound show the property that their displacement errors are maximally negatively correlated. Hence, the combination of two complementary hypotheses is more efficient than two independent hypotheses.
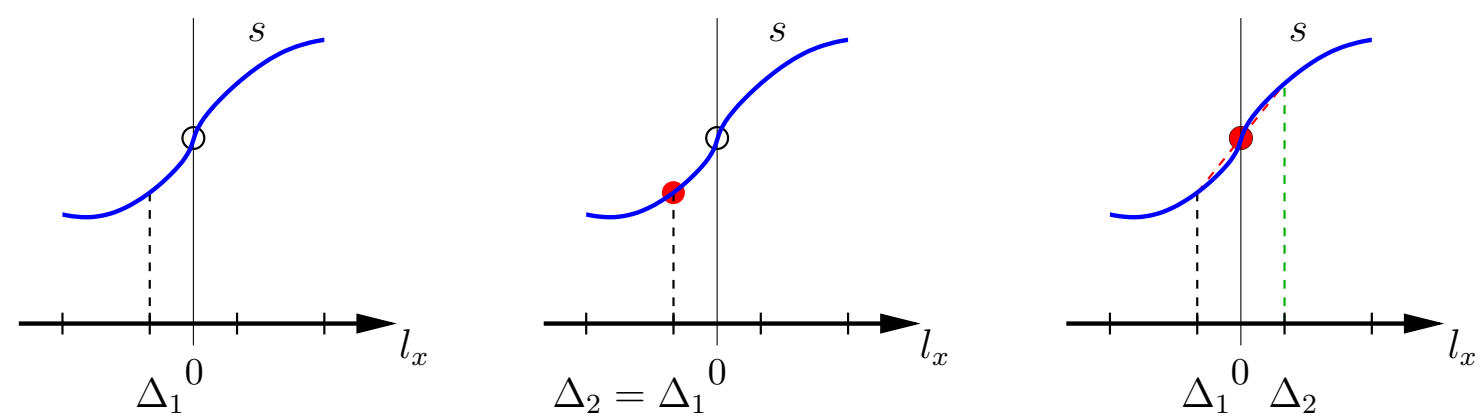

Figure 3.2: Interpolation and displacement error. Due to an inaccurate displacement, only the signal value at spatial location $\Delta_{1}$ is available (left). Averaging two hypotheses with identical displacement errors does not improve the approximation (middle). When we pick the signal value at spatial location $\Delta_{2}=-\Delta_{1}$ and average the two signal values, we will get closer to the signal value at spatial location $l_{x}=0$ (right).

Let us consider the one-dimensional example in Fig. 3.2 where the intensity signal $s$ is a continuous function of the spatial location $l_{x}$. A signal value that we want to use for prediction is given at spatial location $l_{x}=0$. Due to an inaccurate displacement, only the signal value at spatial location $l_{x}=\Delta_{1}$ is available. We assume that the intensity signal is smooth around $l_{x}=0$ and not spatially constant. When we pick the signal value at spatial location $l_{x}=\Delta_{2}=-\Delta_{1}$ and average the two signal values, we will get closer to the signal value at spatial location $l_{x}=0$. If we consider many displacement error values $\Delta_{1}$ with distribution $p_{\boldsymbol{\Delta}}$, we get for the random variables $\boldsymbol{\Delta}_{1}=-\boldsymbol{\Delta}_{2}$. This results in $\rho_{\Delta}=-1$.

Fig. 3.3 depicts the rate difference $\Delta R$ for multihypothesis motion-compensated prediction over the displacement inaccuracy $\beta$ for statistically independent displacement errors according to [Gir00]. The rate difference according to (2.42) represents the maximum bit-rate reduction (in bits/sample) possible by optimal encoding of the prediction error e, compared to optimum intra-frame encoding of the signal s for Gaussian wide-sense stationary signals for the same mean square reconstruction error. A negative rate difference $\Delta R$ corresponds to a reduced bit-rate compared to optimum intra-frame coding. The 


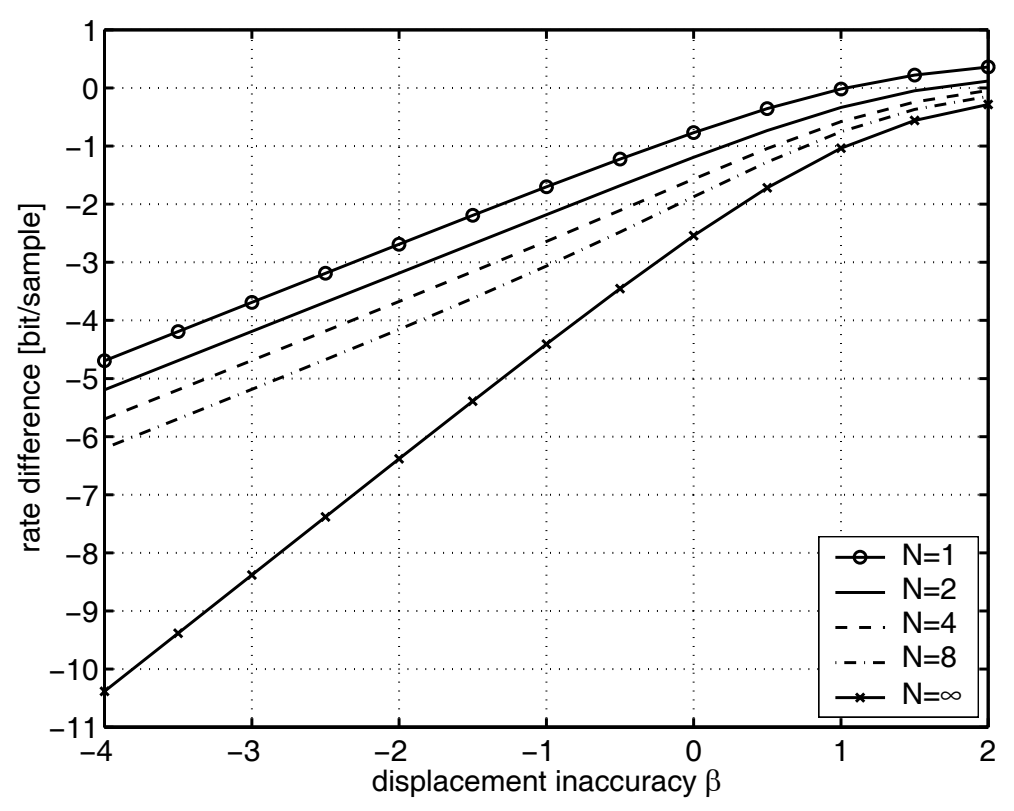

Figure 3.3: Rate difference for superimposed MCP over the displacement inaccuracy $\beta$ for statistically independent displacement errors. The hypotheses are averaged and no residual noise is assumed.

horizontal axis in Fig. 3.3 is calibrated by $\beta=\log _{2}\left(\sqrt{12} \sigma_{\Delta}\right)$, where $\beta=0$ for integer-pel accuracy, $\beta=-1$ for half-pel accuracy, $\beta=-2$ for quarter-pel accuracy, etc [Gir00]. The displacement error variance is given by $(2.34)$.

We observe in Fig. 3.3 that for the case $N=1$ the slope reaches 1 bit per sample per inaccuracy step. This can also be observed in (3.9) for $N=1$ when we apply a Taylor series expansion of first order for the function $P$.

$$
\frac{\Phi_{\mathbf{e e}}(\omega)}{\Phi_{\mathbf{s s}}(\omega)} \approx \sigma_{\Delta}^{2} \omega^{T} \omega \quad \text { for } \quad \sigma_{\Delta}^{2} \rightarrow 0, N=1
$$

Inserting this result in (2.42) supports the observation in Fig. 3.3

$$
\Delta R \approx \beta+\text { const. for } \sigma_{\Delta}^{2} \rightarrow 0, N=1 .
$$

We observe also in Fig. 3.3 that doubling the number of hypotheses decreases the bitrate up to 0.5 bits per sample and the slope reaches up to 1 bit per sample per inaccuracy step. The case $N \rightarrow \infty$ achieves a slope up to 2 bits per sample per inaccuracy step. This can also be observed in (3.9) for $N \rightarrow \infty$ when we apply a Taylor series expansion of second order for the function $P$

$$
\frac{\Phi_{\mathrm{ee}}(\omega)}{\Phi_{\mathrm{ss}}(\omega)} \approx \frac{1}{4} \sigma_{\Delta}^{4}\left(\omega^{T} \omega\right)^{2} \quad \text { for } \quad \sigma_{\boldsymbol{\Delta}}^{2} \rightarrow 0, N \rightarrow \infty, \rho_{\Delta}=0 .
$$

Inserting this result in (2.42) supports the observation in Fig. 3.3

$$
\Delta R \approx 2 \beta+\text { const. for } \quad \sigma_{\Delta}^{2} \rightarrow 0, N \rightarrow \infty, \rho_{\Delta}=0 .
$$




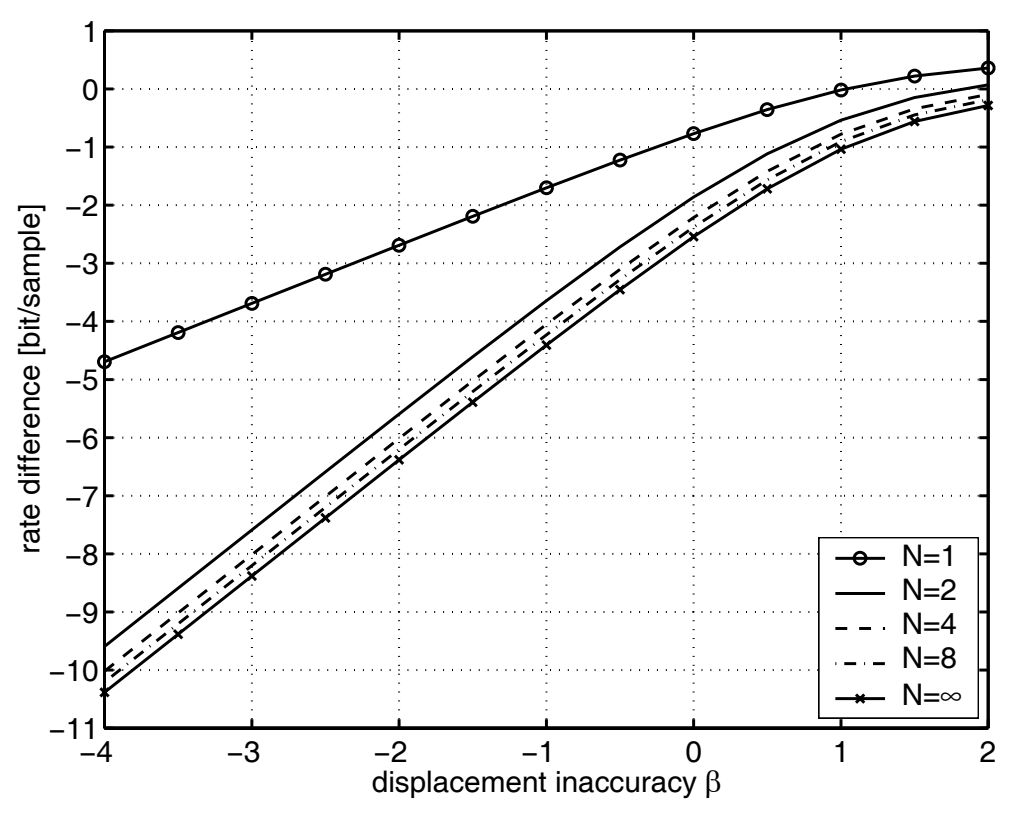

Figure 3.4: Rate difference for superimposed MCP over the displacement inaccuracy $\beta$ for optimized displacement error correlation. The hypotheses are averaged and no residual noise is assumed.

Fig. 3.4 depicts the rate difference for superimposed motion-compensated prediction over the displacement inaccuracy $\beta$ for optimized displacement error correlation according to (3.16). We observe for accurate motion compensation that the slope of the rate difference of 2 bits per sample per inaccuracy step is already reached for $N=2$. For increasing number of hypotheses the rate difference converges to the case $N \rightarrow \infty$ at constant slope. This can also be observed in (3.9) when the displacement error correlation coefficient is set to $\rho_{\boldsymbol{\Delta}}=\frac{1}{1-N}$, and a Taylor series expansion of second order for the function $P(\omega)$ is applied.

$$
\frac{\Phi_{\mathbf{e e}}(\omega)}{\Phi_{\mathbf{s s}}(\omega)} \approx \frac{1}{4} \sigma_{\Delta}^{4}\left(\omega^{T} \omega\right)^{2} \frac{N+1}{N-1} \quad \text { for } \quad \sigma_{\Delta}^{2} \rightarrow 0, N=2,3, \ldots
$$

Inserting this result in 2.42 supports the observation in Fig. 3.4 that for $N=2,3, \ldots$ the slope reaches up to 2 bits per sample per inaccuracy step.

$$
\Delta R \approx 2 \beta+\frac{1}{2} \log _{2}\left(\frac{N+1}{N-1}\right)+\text { const. for } \quad \sigma_{\Delta}^{2} \rightarrow 0, N=2,3, \ldots
$$

For very accurate motion compensation, doubling the number of hypotheses results in a rate difference of

$$
\Delta R_{2}:=\Delta R(2 N)-\Delta R(N) \approx \frac{1}{2} \log _{2}\left(\frac{2 N^{2}-N-1}{2 N^{2}+N-1}\right) \quad \text { for } \quad \sigma_{\Delta}^{2} \rightarrow 0, N=2,3, \ldots
$$

For a very large number of hypotheses, the rate difference for doubling the number of hypotheses $\Delta R_{2}$ converges to zero. Consequently, the prediction gain by optimum noiseless multihypotheses prediction with averaging filter is limited and the rate difference converges to the case $N \rightarrow \infty$ at constant slope. 
We obtain for the band-limited frame signal the following result: the gain of superimposed motion-compensated prediction with jointly optimal motion estimation over motion-compensated prediction increases by improving the accuracy of motion compensation for each hypothesis. The theoretical results suggest that a practical video coding algorithm should utilize two jointly estimated hypotheses. Experimental results also suggest that the gain by superimposed prediction is limited and that two jointly estimated hypotheses provide a major portion of this achievable gain.

\subsubsection{Gradient of the Prediction Error Variance}

We observe in Fig. 3.1 that the prediction error variance $\sigma_{\mathbf{e}}^{2}$ increases monotonically for increasing displacement error correlation coefficient $\rho_{\Delta}$. In the following, we investigate this in more detail and show that this dependency is independent of a particular frame signal model, i.e., a particular frame autocorrelation function.

Again, let $\mathbf{s}(l)$ and $\mathbf{c}_{\mu}(l)$ be generated by a jointly wide-sense stationary, two-dimensional random process. The vector $l=(x, y)^{T}$ denotes the location in $\mathcal{R}^{2}$. Let $\mathbf{c}_{\mu}$ be the $\mu$-th of $N$ motion-compensated signals available for prediction, and $\mathbf{s}$ be the current frame to be predicted. The limited accuracy of motion compensation is captured by associating a vector-valued displacement error $\boldsymbol{\Delta}_{\mu}$ with the $\mu$-th hypothesis $\mathbf{c}_{\mu}$. For simplicity, we assume that all hypotheses are shifted versions of the current frame signal $\mathbf{s}$. The shift is determined by the vector-valued displacement error $\boldsymbol{\Delta}_{\mu}$ of the $\mu$-th hypotheses such that $\mathbf{c}_{\mu}(l)=\mathbf{s}\left(l-\boldsymbol{\Delta}_{\mu}\right)$.

The superimposed motion-compensated predictor forms a prediction signal by averaging $N$ hypotheses $\mathbf{c}_{\mu}(l)$ in order to predict the current frame signal $\mathbf{s}(l)$. The prediction error at location $l$ is the difference between the current frame signal and $N$ averaged hypotheses

$$
\mathbf{e}(l)=\mathbf{s}(l)-\frac{1}{N} \sum_{\mu=1}^{N} \mathbf{s}\left(l-\Delta_{\mu}\right) .
$$

As we assume wide-sense stationary signals, the prediction error variance $\sigma_{\mathbf{e}}^{2}=E\left\{\mathbf{e}^{2}(l)\right\}$ is independent of the location $l$

$$
\sigma_{\mathbf{e}}^{2}=\sigma_{\mathbf{s}}^{2}-\frac{2}{N} \sum_{\mu=1}^{N} E\left\{\phi_{\mathbf{s s}}\left(\boldsymbol{\Delta}_{\mu}\right)\right\}+\frac{1}{N^{2}} \sum_{\mu=1}^{N} \sum_{\nu=1}^{N} E\left\{\phi_{\mathbf{s s}}\left(\boldsymbol{\Delta}_{\mu}-\boldsymbol{\Delta}_{\nu}\right)\right\}
$$

but is dependent on the scalar space-continuous autocorrelation function

$$
\phi_{\mathbf{s} \mathbf{s}}(l)=E\left\{\mathbf{s}\left(l_{0}+l\right) \mathbf{s}^{*}\left(l_{0}\right)\right\} .
$$

The autocorrelation function of a wide-sense stationary random process does not depend on the absolute location $l_{0}$ but only on the relative two-dimensional shift $l$.

For the $\mu$-th displacement error $\boldsymbol{\Delta}_{\mu}$, a 2-D stationary normal distribution with variance $\sigma_{\Delta}^{2}$ and zero mean is assumed where the $x$ - and $y$-components are statistically independent. The displacement error variance is the same for all $N$ hypotheses. Further, the pairs 
$\left(\boldsymbol{\Delta}_{\mu}, \boldsymbol{\Delta}_{\nu}\right)$ are assumed to be jointly Gaussian random variables. The correlation coefficient $\rho_{\boldsymbol{\Delta}}$ between two displacement error components $\boldsymbol{\Delta}_{\mathbf{x} \mu}$ and $\boldsymbol{\Delta}_{\mathbf{x} \nu}$ is the same for all pairs of hypotheses. With these assumptions, the covariance matrix of a displacement error component is given by (3.5). Since the covariance matrix is nonnegative definite, the correlation coefficient $\rho_{\boldsymbol{\Delta}}$ has the limited range according to (3.6).

For the expected values in $(3.25)$, we define a function $g\left(\omega_{0}, \sigma_{\Delta}^{2}\right)$ that is only dependent on the spatial correlation of the frame signal $\rho_{\mathbf{s}}=\exp \left(-\omega_{0}\right)$ and the displacement error variance $\sigma_{\boldsymbol{\Delta}}^{2}$. Further, we exploit the fact that the difference of two jointly Gaussian random variables is also Gaussian.

$$
\begin{aligned}
E\left\{\phi_{\mathbf{s s}}\left(\boldsymbol{\Delta}_{\mu}\right)\right\} & =\sigma_{s}^{2} g\left(\omega_{0}, \sigma_{\boldsymbol{\Delta}}^{2}\right) \text { for } \mu=1,2, \ldots, N \\
E\left\{\phi_{\mathbf{s s}}\left(\boldsymbol{\Delta}_{\mu}-\boldsymbol{\Delta}_{\nu}\right)\right\} & =\sigma_{s}^{2} g\left(\omega_{0}, 2 \sigma_{\boldsymbol{\Delta}}^{2}\left(1-\rho_{\boldsymbol{\Delta}}\right)\right) \text { for } \mu \neq \nu
\end{aligned}
$$

With (3.27) and (3.28), the prediction error variance in (3.25) can be normalized to the frame signal variance $\sigma_{\mathbf{s}}^{2}$. Note that for $\mu=\nu$ the expected value $E\left\{\phi_{\mathbf{s s}}\left(\boldsymbol{\Delta}_{\mu}-\boldsymbol{\Delta}_{\nu}\right)\right\}$ is equal to the variance of the frame signal $\sigma_{\mathbf{s}}^{2}$.

$$
\frac{\sigma_{\mathbf{e}}^{2}}{\sigma_{\mathbf{s}}^{2}}=\frac{N+1}{N}-2 g\left(\omega_{0}, \sigma_{\boldsymbol{\Delta}}^{2}\right)+\frac{N-1}{N} g\left(\omega_{0}, 2 \sigma_{\boldsymbol{\Delta}}^{2}\left(1-\rho_{\boldsymbol{\Delta}}\right)\right) \quad \text { for } \quad \frac{1}{1-N} \leq \rho_{\boldsymbol{\Delta}} \leq 1
$$

With the function $g$ in (3.27), we can show that for $N>1$ the prediction error variance $\sigma_{\mathbf{e}}^{2}$ increases monotonically with the displacement error correlation coefficient $\rho_{\boldsymbol{\Delta}}$. We know for the single hypothesis predictor that the prediction error variance increases monotonically with the displacement error variance $\sigma_{\boldsymbol{\Delta}}^{2}$. For $N=1$, (3.29) implies

$$
\frac{\partial \sigma_{\mathbf{e}}^{2}}{\partial \sigma_{\boldsymbol{\Delta}}^{2}}=-2 \sigma_{\mathbf{s}}^{2} \frac{\partial g\left(\omega_{0}, \sigma_{\boldsymbol{\Delta}}^{2}\right)}{\partial \sigma_{\boldsymbol{\Delta}}^{2}}>0
$$

that is, the partial derivative of $g$ with respect to $\sigma_{\Delta}^{2}$ is negative. Next, we calculate the partial derivative of the prediction error variance with respect to the displacement error correlation coefficient $\rho_{\boldsymbol{\Delta}}$ for $N>1$ and observe that this partial derivative is positive.

$$
\frac{\partial \sigma_{\mathbf{e}}^{2}}{\partial \rho_{\boldsymbol{\Delta}}}=-2 \sigma_{\boldsymbol{\Delta}}^{2} \sigma_{\mathbf{s}}^{2} \frac{N-1}{N} \frac{\partial g\left(\omega_{0}, 2 \sigma_{\boldsymbol{\Delta}}^{2}\left(1-\rho_{\boldsymbol{\Delta}}\right)\right)}{\partial 2 \sigma_{\boldsymbol{\Delta}}^{2}\left(1-\rho_{\boldsymbol{\Delta}}\right)}>0
$$

Consequently, the prediction error variance $\sigma_{\mathbf{e}}^{2}$ increases monotonically with the displacement error correlation coefficient $\rho_{\Delta}$ independent of a particular underlying frame signal model.

\subsection{Hypotheses with Additive Noise}

To consider signal components that cannot be modeled by motion compensation, statistically independent noise $\mathbf{n}_{\mu}$ is added to each motion-compensated signal. Further, we assume that the current frame $\mathbf{s}$ originates from a noise-free model video signal $\mathbf{v}$ 
but is also characterized by statistically independent additive Gaussian noise $\mathbf{n}_{0}$ [Gir00] We characterize the residual noise $\mathbf{n}_{\mu}$ for each hypothesis by the power spectal density $\Phi_{\mathbf{n}_{\mu} \mathbf{n}_{\mu}}(\omega)$ and the residual noise $\mathbf{n}_{0}$ in the current frame by $\Phi_{\mathbf{n}_{0} \mathbf{n}_{0}}(\omega)$. For convenience, we normalize the noise power spectra with respect to the power spectral density of the model video signal $\Phi_{\mathbf{v v}}(\omega)$.

$$
\alpha_{\mu}(\omega)=\frac{\Phi_{\mathbf{n}_{\mu} \mathbf{n}_{\mu}}(\omega)}{\Phi_{\mathbf{v v}}(\omega)} \quad \forall \mu=0,1,2, \ldots, N
$$

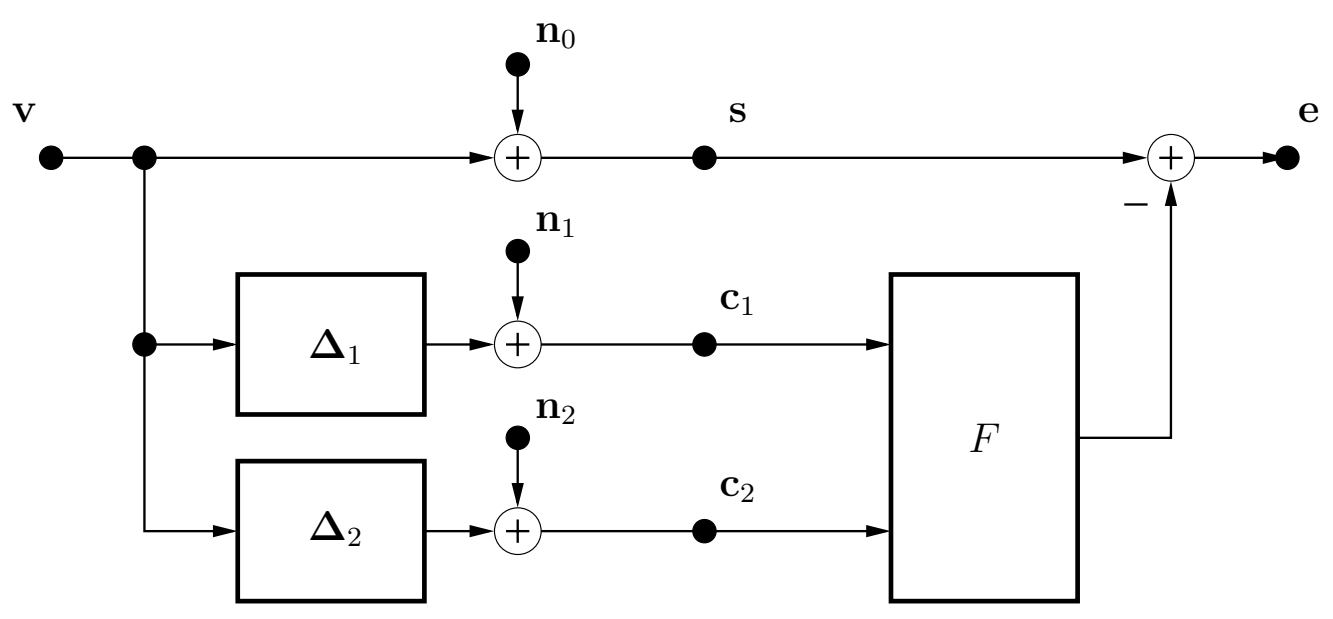

Figure 3.5: Two-hypothesis motion-compensated prediction with linear filter.

Fig. 3.5 depicts the model for motion-compensated signals with statistically independent additive noise and linear filter. All hypotheses are jointly filtered to determine the final prediction signal. The linear filter is described by the vector valued transfer function $F(\omega)$. In particular, $F(\omega)$ is a row vector with $N$ scalar transfer functions. The power spectrum of the prediction error with the linear filter is

$$
\Phi_{\mathbf{e e}}(\omega)=\Phi_{\mathbf{s s}}(\omega)-\Phi_{\mathbf{s c}}(\omega) F^{H}(\omega)-F(\omega) \Phi_{\mathbf{c s}}(\omega)+F(\omega) \Phi_{\mathbf{c c}}(\omega) F^{H}(\omega) .
$$

In the following, we investigate the performance of motion compensation with complementary hypotheses for both the averaging filter and a Wiener filter.

\subsubsection{Averaging Filter}

The averaging filter weights each hypothesis equally with the constant factor $\frac{1}{N}$. We use the notation of the column vector 1 denoting that all entries are equal to one.

$$
F(\omega)=\frac{1}{N} \mathbf{1}^{T}
$$

We evaluate the performance of motion compensation with complementary hypotheses for the averaging filter in (3.34) by calculating the rate difference in (2.42). In order to do 
this, we normalize the power spectral density of the prediction error and substitute the power spectra in (3.33) with (3.3), (3.4), (3.7) and (3.8). Further, we assume individual power spectral densities for the residual noise.

$$
\frac{\Phi_{\mathbf{e e}}(\omega)}{\Phi_{\mathrm{ss}}(\omega)}=1+\frac{N+\sum_{\mu=1}^{N} \alpha_{\mu}(\omega)}{N^{2}\left(1+\alpha_{0}(\omega)\right)}-\frac{2 P\left(\omega, \sigma_{\Delta}^{2}\right)}{1+\alpha_{0}(\omega)}+\frac{N-1}{N} \cdot \frac{P\left(\omega, 2 \sigma_{\Delta}^{2}\left(1-\rho_{\boldsymbol{\Delta}}\right)\right)}{1+\alpha_{0}(\omega)}
$$

Experimental results for the predictor design in [Fli97a, FWG98] show that all $N$ hypotheses contribute equally well. Based on this observation, we assume that the noise power spectral densities are identical for all $N$ hypotheses. To simplify the model, we assume also that they are identical to the noise power spectrum of the current frame, i.e., $\alpha_{\mu}(\omega)=\alpha_{0}(\omega)$ for $\mu=1,2, \ldots, N$. With these assumptions, the normalized prediction error power spectrum for the superimposed predictor with averaging filter reads

$$
\frac{\Phi_{\mathbf{e e}}(\omega)}{\Phi_{\mathrm{ss}}(\omega)}=\frac{N+1}{N}-\frac{2 P\left(\omega, \sigma_{\boldsymbol{\Delta}}^{2}\right)}{1+\alpha_{0}(\omega)}+\frac{N-1}{N} \cdot \frac{P\left(\omega, 2 \sigma_{\boldsymbol{\Delta}}^{2}\left(1-\rho_{\boldsymbol{\Delta}}\right)\right)}{1+\alpha_{0}(\omega)} .
$$

If there is no noise, i.e., $\alpha_{0}(\omega)=0$, we obtain the result in (3.9).

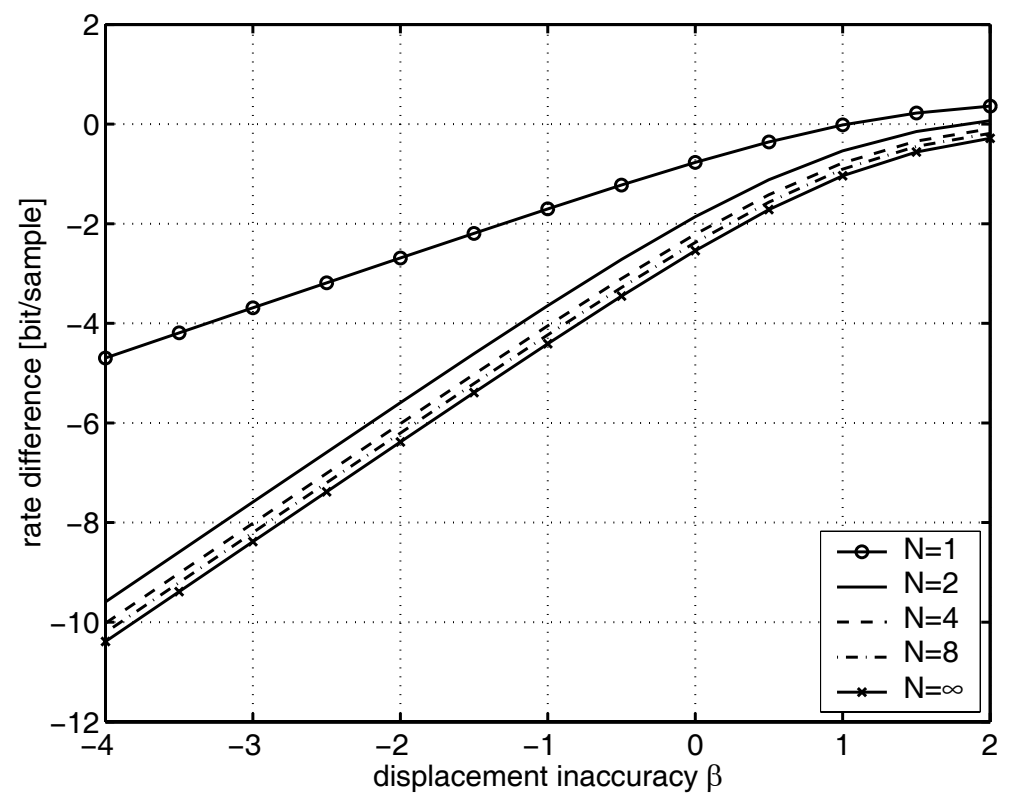

Figure 3.6: Rate difference for motion compensation with complementary hypotheses and averaging filter over the displacement inaccuracy $\beta$. Residual noise level RNL $=-100 \mathrm{~dB}$.

Figs. 3.6 and 3.7 depict the rate difference for motion compensation with complementary hypotheses over the displacement inaccuracy $\beta$ at a residual noise level of $-100 \mathrm{~dB}$ and $-30 \mathrm{~dB}$, respectively. The residual noise level is defined by $\mathrm{RNL}=10 \log _{10}\left(\sigma_{\mathbf{n}}^{2}\right)$ where $\sigma_{\mathbf{n}}^{2}$ is the residual noise variance. As suggested in [Gir00], we assume a constant power spectral density for the residual noise. For the plotted range of the motion inaccuracy, a residual noise level of $\mathrm{RNL}=-100 \mathrm{~dB}$ indicates that the residual noise is negligible and the performance is similar to the noiseless case as shown in Fig. 3.4. At a residual noise level 


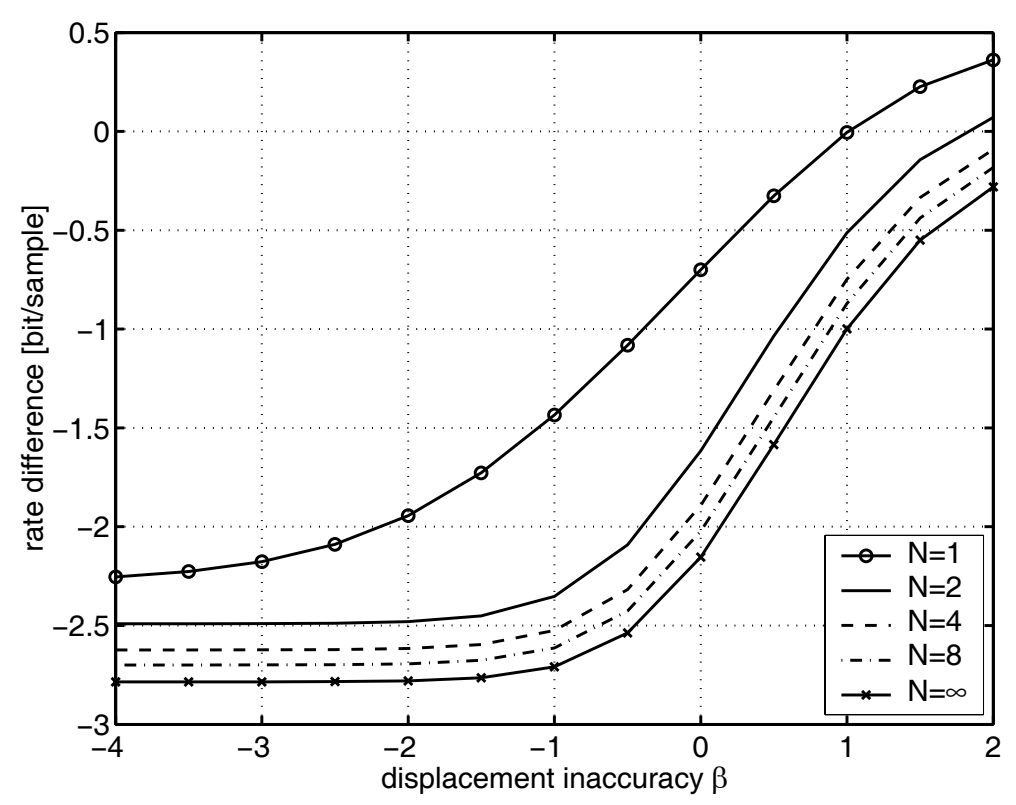

Figure 3.7: Rate difference for motion compensation with complementary hypotheses and averaging filter over the displacement inaccuracy $\beta$. Residual noise level RNL $=-30 \mathrm{~dB}$.

of $\mathrm{RNL}=-30 \mathrm{~dB}$, the rate difference saturates beyond $1 / 8$-th pel accuracy $(\beta=-3)$. This is more practical. We observe for motion compensation with complementary hypotheses that the rate difference saturates even at quarter-pel accuracy. Consequently, we can achieve similar prediction performance at lower compensation accuracy when utilizing motion compensation with complementary hypotheses. Regardless of the accuracy of superimposed motion compensation, the rate difference improves for an increasing number of hypotheses due to the noise suppression by the averaging filter.

\subsubsection{Wiener Filter}

The optimum Wiener filter minimizes the power spectral density of the prediction error. This is a well-known result [Pap91] and we adopt the expression for multihypothesis motion-compensation from [Gir00]. The vector-valued optimum filter $F_{o}(\omega)$ is the product of the Hermitian conjugate of the cross spectrum, $\Phi_{\mathbf{c s}}^{H}(\omega)$, and the inverse power spectral density matrix of the hypotheses $\Phi_{\mathbf{c c}}^{-1}(\omega)$.

$$
F_{o}(\omega)=\Phi_{\mathbf{c s}}^{H}(\omega) \Phi_{\mathbf{c c}}^{-1}(\omega)
$$

This expression minimizes the power spectral density of the prediction error and its minimum is given by

$$
\Phi_{\mathbf{e e}}(\omega)=\Phi_{\mathbf{s s}}(\omega)-\Phi_{\mathbf{c s}}^{H}(\omega) \Phi_{\mathbf{c c}}^{-1}(\omega) \Phi_{\mathbf{c s}}(\omega) .
$$

To evaluate the performance of the predictor with optimum Wiener filter, we have to specify the vector-valued cross spectrum $\Phi_{\mathbf{c s}}(\omega)$ and the power spectral density matrix of the hypotheses $\Phi_{\mathbf{c c}}(\omega)$. In the following, we analyze the influence of motion compensation with complementary hypotheses on both the Wiener filter and its prediction performance. 
For motion compensation with complementary hypotheses and (3.7), the vector of the cross spectra is simply

$$
\Phi_{\mathbf{c s}}(\omega)=1 P\left(\omega, \sigma_{\Delta}^{2}\right) \Phi_{\mathbf{v v}}(\omega) .
$$

The cross spectra do not include the power spectral densities of the residual noise as we assume that the individual noise signals are mutually statistically independent. The same argument holds for the non-diagonal entries in the matrix $\Phi_{\mathbf{c c}}$ which are determined by the characteristic function of the displacement error PDF with the displacement error correlation coefficient $\rho_{\boldsymbol{\Delta}}$ according to (3.8). The diagonal entries in the matrix $\Phi_{\mathbf{c c}}$ are characterized by the power spectral densities of the hypotheses which include the power spectral densities of the residual noise. We write the matrix $\Phi_{\mathbf{c c}}$ as the sum of the matrix $\mathbf{1 1}^{T}$ and the diagonal matrix $\operatorname{diag}(\cdot)$ as this representation is useful for the following discussion. $\mathbf{1 1}^{T}$ is the $N \times N$ matrix with all entries equal to one. With that, the power spectral density matrix for $N$ hypotheses becomes

$$
\Phi_{\mathbf{c c}}(\omega)=\left[11^{T}+\operatorname{diag}\left(\frac{1+\alpha_{i}(\omega)}{P_{\rho}(\omega)}-1\right)\right] P_{\rho}(\omega) \Phi_{\mathbf{v v}}(\omega)
$$

where $P_{\rho}(\omega)=P\left(\omega, 2 \sigma_{\boldsymbol{\Delta}}^{2}\left(1-\rho_{\boldsymbol{\Delta}}\right)\right)$ abbreviates the characteristic function of the displacement error PDF with the displacement error correlation coefficient $\rho_{\boldsymbol{\Delta}} \cdot \alpha_{i}(\omega)$ represents the normalized power spectral density of the residual noise in the $i$-th hypothesis.

The Wiener solution requires the inverse of the power spectral density matrix $\Phi_{\mathbf{c c}}(\omega)$. An analytical expression is derived in Appendix A.3 and the optimum Wiener filter according to (3.37) yields

$$
F_{o}(\omega)=\frac{1}{\mathbf{1}^{T} b(\omega)-1} \cdot \frac{P\left(\omega, \sigma_{\Delta}^{2}\right)}{P_{\rho}(\omega)} b^{T}(\omega)
$$

with the vector

$$
b^{T}(\omega)=\left(\frac{-P_{\rho}(\omega)}{1+\alpha_{1}(\omega)-P_{\rho}(\omega)}, \frac{-P_{\rho}(\omega)}{1+\alpha_{2}(\omega)-P_{\rho}(\omega)}, \ldots, \frac{-P_{\rho}(\omega)}{1+\alpha_{N}(\omega)-P_{\rho}(\omega)}\right) .
$$

Please note that only the normalized noise spectrum $\alpha_{\mu}(\omega)$ differs in the components of $b(\omega)$ and that the contribution from inaccurate motion compensation is the same in each component. The expression for the power spectral density of the prediction error in (3.38) incorporates explicitly the solution for the Wiener filter in (3.37) such that $\Phi_{\mathbf{e e}}=\Phi_{\mathbf{s s}}-F_{o} \Phi_{\mathbf{c s}}$. After normalization with $\Phi_{\mathbf{s s}}(\omega)=\left(1+\alpha_{0}(\omega)\right) \Phi_{\mathbf{v v}}(\omega)$, the power spectral density of the prediction error reads

$$
\frac{\Phi_{\mathbf{e e}}(\omega)}{\Phi_{\mathbf{s}}(\omega)}=1-\frac{1}{1+\alpha_{0}(\omega)} \cdot \frac{\mathbf{1}^{T} b(\omega)}{\mathbf{1}^{T} b(\omega)-1} \cdot \frac{P^{2}\left(\omega, \sigma_{\boldsymbol{\Delta}}^{2}\right)}{P_{\rho}(\omega)} .
$$

As a reminder, $\alpha_{0}(\omega)$ denotes the normalized power spectral density of the residual noise in the current frame. 
If the noise energy in all the hypotheses and the current frame is the same, that is, $\alpha_{\mu}(\omega)=\alpha(\omega)$ for $\mu=0,1, \ldots, N$, we obtain for the optimum Wiener filter

$$
F_{o}(\omega)=\frac{P\left(\omega, \sigma_{\Delta}^{2}\right)}{1+\alpha(\omega)+(N-1) P_{\rho}(\omega)} \mathbf{1}^{T},
$$

and for the normalized power spectral density of the prediction error

$$
\frac{\Phi_{\mathbf{e e}}(\omega)}{\Phi_{\mathbf{s}}(\omega)}=1-\frac{1}{1+\alpha(\omega)} \cdot \frac{N P^{2}\left(\omega, \sigma_{\Delta}^{2}\right)}{1+\alpha(\omega)+(N-1) P_{\rho}(\omega)} .
$$

Based on this result, we investigate the influence of motion compensation with complementary hypotheses on both the Wiener filter and its prediction performance.

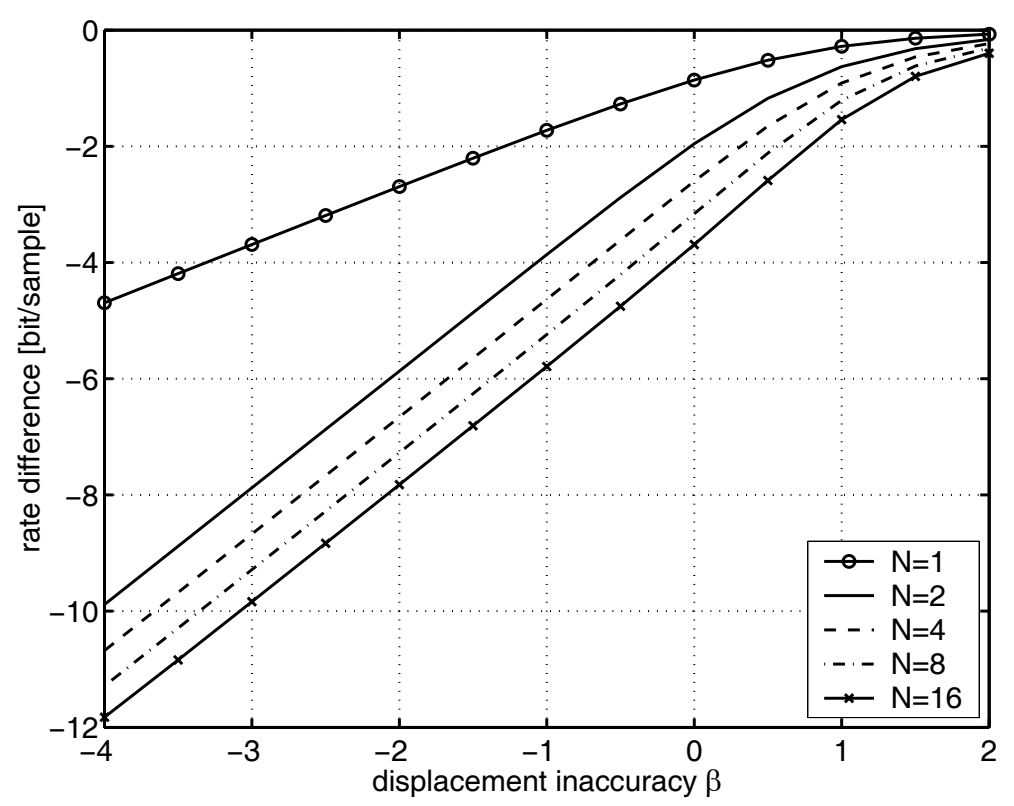

Figure 3.8: Rate difference for motion compensation with complementary hypotheses and Wiener filter over the displacement inaccuracy $\beta$. Residual noise level RNL $=-100 \mathrm{~dB}$.

Fig. 3.8 depicts the rate difference for motion compensation with complementary hypotheses and Wiener filter over the displacement inaccuracy $\beta$ and negligible residual noise. For $N>1$, the graphs show that doubling the number of complementary hypotheses decreases the bit-rate at least by 0.5 bits per sample and the slope reaches up to 2 bits per sample per inaccuracy step. This can also be observed in (3.45) when the residual noise is neglected, i.e. $\alpha(\omega) \rightarrow 0$, the displacement error correlation coefficient is set to $\rho_{\boldsymbol{\Delta}}=\frac{1}{1-N}$, and a Taylor series expansion of second order is applied for the function $P\left(\omega, \sigma_{\Delta}^{2}\right)$.

$$
\frac{\Phi_{\mathrm{ee}}(\omega)}{\Phi_{\mathrm{ss}}(\omega)} \approx \frac{1}{2} \sigma_{\boldsymbol{\Delta}}^{4}\left(\omega^{T} \omega\right)^{2} \frac{1}{N-1} \quad \text { for } \quad \sigma_{\boldsymbol{\Delta}}^{2} \rightarrow 0
$$

Inserting this result into 2.42 supports the observation in Fig. 3.8 that the slope reaches up to 2 bits per sample per inaccuracy step

$$
\Delta R \approx 2 \beta+\frac{1}{2} \log _{2}\left(\frac{1}{N-1}\right)+\text { const. for } \quad \sigma_{\Delta}^{2} \rightarrow 0 .
$$


For very accurate motion compensation, doubling the number of hypotheses results in a rate difference of

$$
\Delta R_{2}:=\Delta R(2 N)-\Delta R(N) \approx \frac{1}{2} \log _{2}\left(\frac{N-1}{2 N-1}\right) \quad \text { for } \quad \sigma_{\Delta}^{2} \rightarrow 0 .
$$

For a very large number of hypotheses, the rate difference for doubling the number of hypotheses $\Delta R_{2}$ converges to -0.5 bits per sample. Consequently, the prediction error variance of the optimal noiseless superimposed predictor with Wiener filter converges to zero for an infinite number of hypotheses.

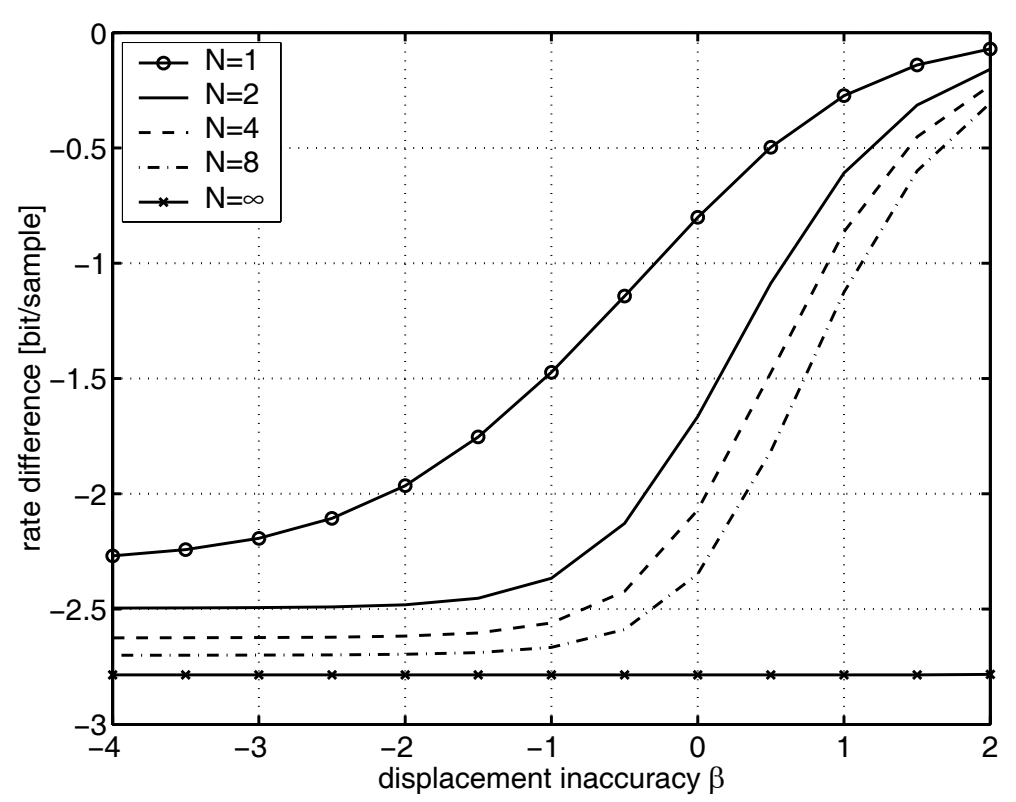

Figure 3.9: Rate difference for motion compensation with complementary hypotheses and Wiener filter over the displacement inaccuracy $\beta$. Residual noise level RNL $=-30 \mathrm{~dB}$.

Fig. 3.9 shows the rate difference for motion compensation with complementary hypotheses and Wiener filter over the displacement inaccuracy $\beta$ at a residual noise level of $-30 \mathrm{~dB}$. Similar to the averaging filter for motion compensation with complementary hypotheses in Fig. 3.7, the rate difference saturates even at for quarter-pel accuracy. And for very accurate superimposed motion compensation, the rate difference improves for an increasing number of hypotheses due to the noise suppression by the Wiener filter but saturates for $N \rightarrow \infty$. In contrast to the averaging filter, the Wiener filter with a very large number of hypotheses is able to eliminate the influence of motion inaccuracy. This can be observed in (3.45) for $N \rightarrow \infty$. In this case, the normalized power spectral density of the prediction error yields

$$
\frac{\Phi_{\mathbf{e e}}(\omega)}{\Phi_{\mathrm{ss}}(\omega)}=1-\frac{1}{1+\alpha(\omega)} \quad \text { for } \quad N \rightarrow \infty
$$

Fig. 3.10 depicts the rate difference for superimposed motion-compensated prediction over the displacement inaccuracy $\beta$ for both optimized displacement error correlation and 


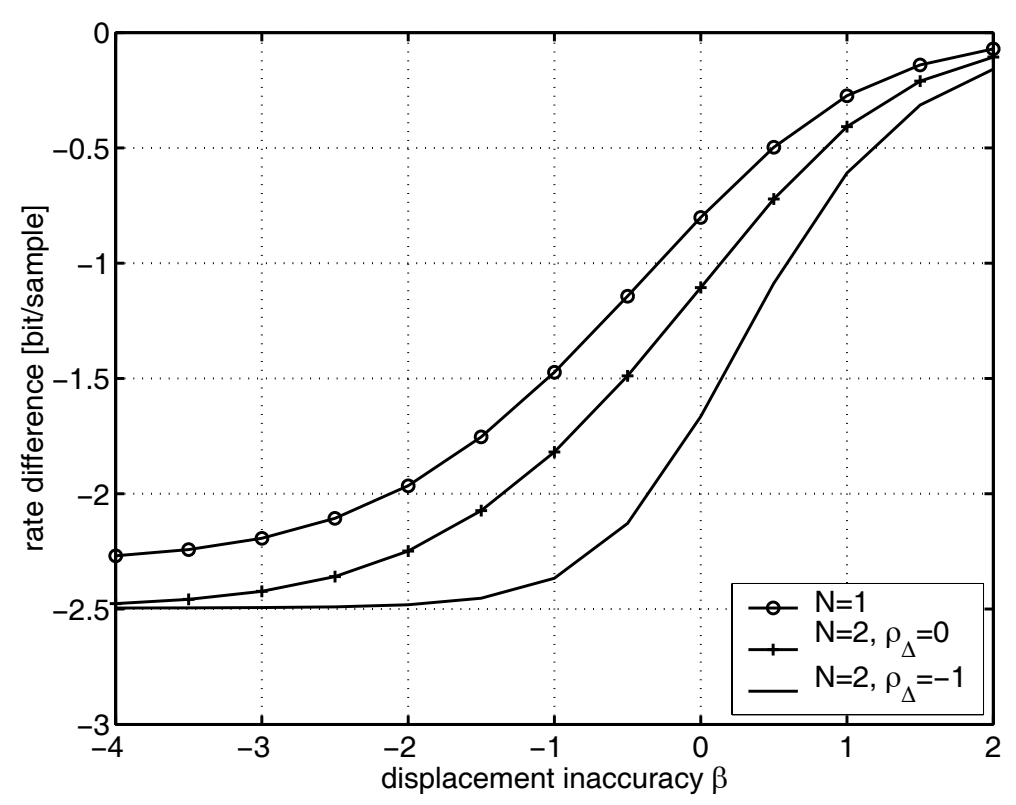

Figure 3.10: Rate difference for superimposed motion-compensated prediction with Wiener filter over the displacement inaccuracy $\beta$ for both optimized displacement error correlation and statistically independent displacement error. Residual noise level RNL $=-30 \mathrm{~dB}$. In all cases, the optimum filter is applied.

statistically independent displacement error. The residual noise level is chosen to be $30 \mathrm{~dB}$. For half-pel accurate motion compensation and 2 hypotheses, we gain about 0.6 bits/sample in rate difference for motion compensation with complementary hypotheses over statistically independent displacement error. This corresponds to a prediction gain of about $3.6 \mathrm{~dB}$.

So far, we investigated the prediction performance achieved by the optimum Wiener filter for motion compensation with complementary hypotheses. In the following, we discuss the transfer function of the optimum Wiener filter according to (3.44) for motion compensation with $N=2$ complementary hypotheses and compare it to that of the optimum Wiener filter for motion compensation with $N=1$ hypothesis.

Figs. 3.11 and 3.12 depict the transfer function of one component of the optimum Wiener filter according to (3.44) for $N=1$ and $N=2$, respectively. In both cases, we neglect the residual noise and use integer-pel accurate motion compensation. The transfer function of the filter for the superimposed predictor is flatter and, hence, shows significantly less spectral selectivity when compared to the case of the single hypothesis predictor. In addition, the transfer function for motion compensation with complementary hypotheses seems to suppress low frequency components. We will investigate this further by means of cross sections of the transfer functions.

Figs. 3.13 and 3.14 show cross sections at $\omega_{y}=0$ of the transfer functions of the optimum Wiener filter according to (3.44) for $\beta=0$ and $\beta=1$, respectively. The onehypothesis filter $N=1$ is compared to the two-hypothesis filter $N=2$ with optimized displacement error correlation coefficient $\rho_{\boldsymbol{\Delta}}=-1$ and uncorrelated displacement error 


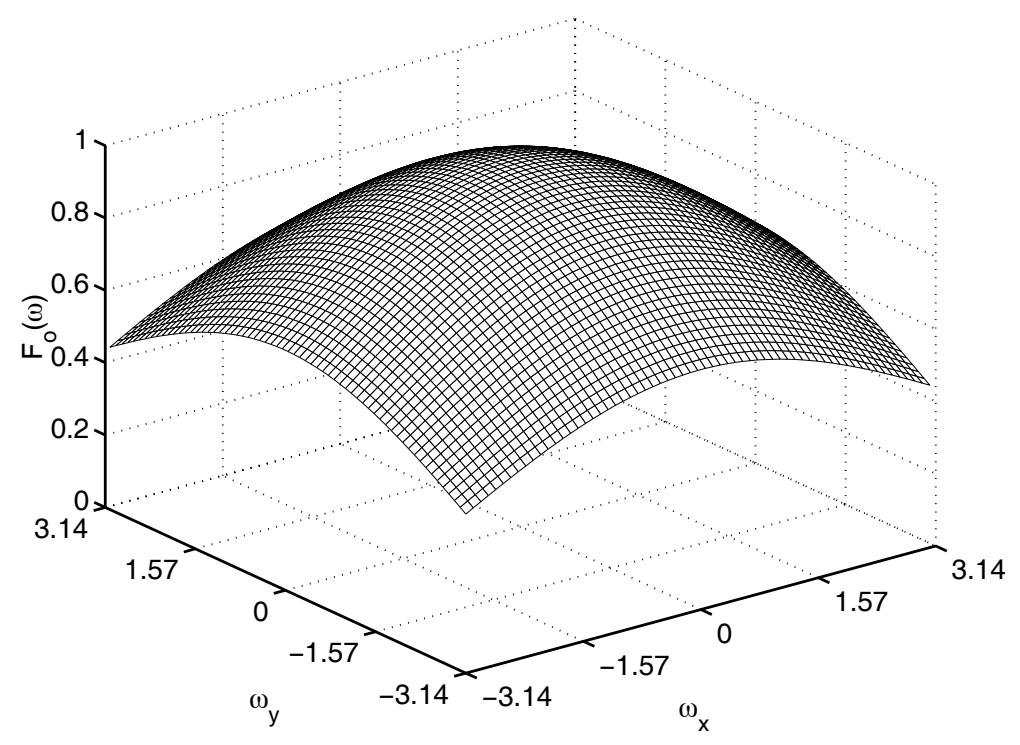

Figure 3.11: Transfer function of the Wiener filter for $N=1, \beta=0$, and no residual noise.

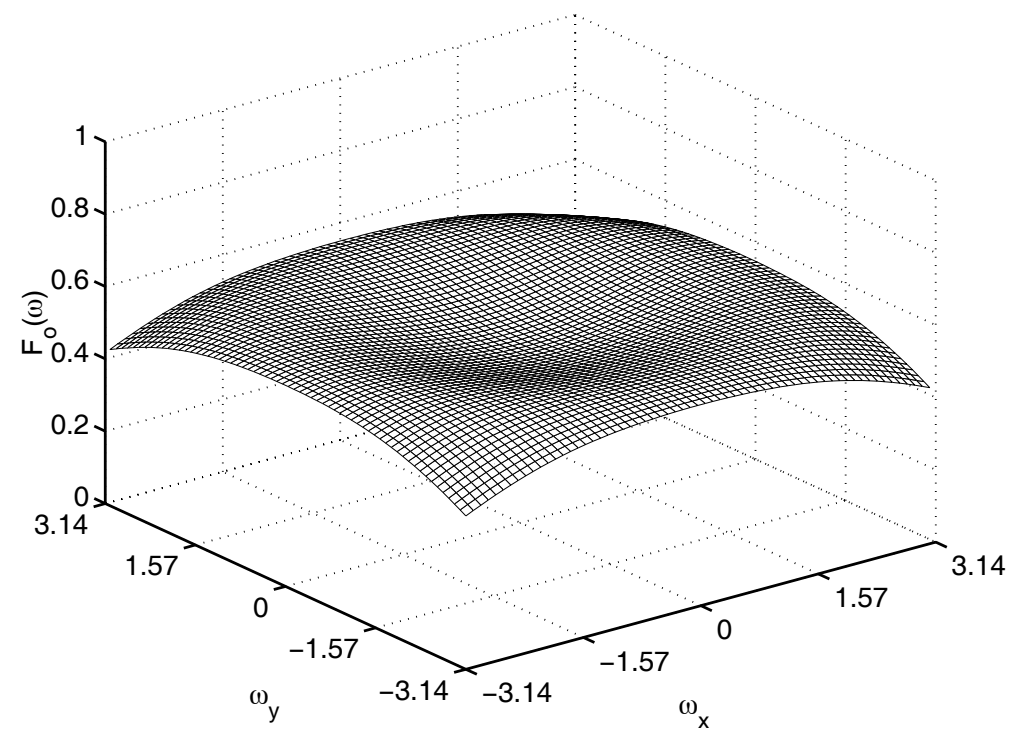

Figure 3.12: Transfer function of a component of the Wiener filter for $N=2, \beta=0$, and no residual noise.

$\rho_{\Delta}=0$. The spectral selectivity decreases for more accurate motion compensation. This allows us to accurately compensate high frequency components. Interestingly, the filter for motion compensation with complementary hypotheses amplifies high frequency components when compared to the two-hypothesis filter with uncorrelated displacement error. In other words, the optimum Wiener filter for motion compensation with complementary hypotheses promotes high frequency components that are otherwise suppressed by the two-hypothesis filter with uncorrelated displacement error. These effects grow larger for less accurate motion compensation. 


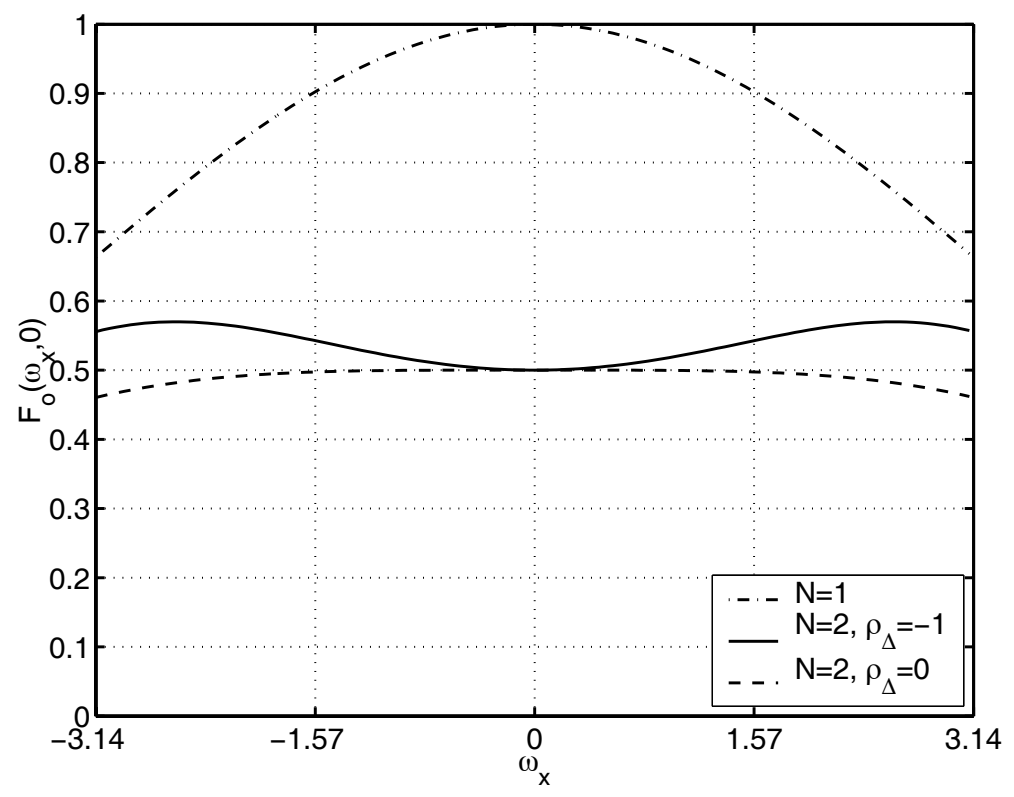

Figure 3.13: Cross section at $\omega_{y}=0$ of the transfer function of the Wiener filter for $\beta=0$ and no residual noise. The one-hypothesis filter $N=1$ is compared to the two-hypothesis filter $N=2$ with optimized displacement error correlation coefficient $\rho_{\boldsymbol{\Delta}}=-1$ and uncorrelated displacement error $\rho_{\boldsymbol{\Delta}}=0$.

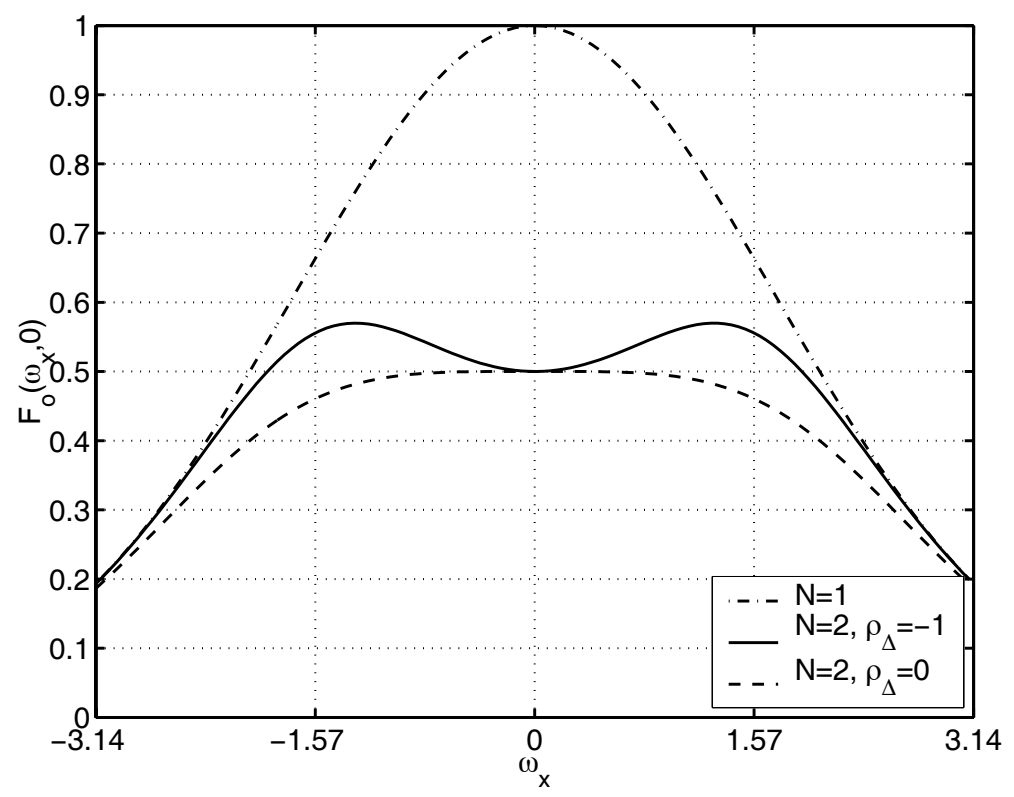

Figure 3.14: Cross section at $\omega_{y}=0$ of the transfer function of the Wiener filter for $\beta=1$ and no residual noise. The one-hypothesis filter $N=1$ is compared to the two-hypothesis filter $N=2$ with optimized displacement error correlation coefficient $\rho_{\boldsymbol{\Delta}}=-1$ and uncorrelated displacement error $\rho_{\boldsymbol{\Delta}}=0$. 
As previously mentioned, very accurate motion compensation flattens the characteristic function of the displacement error PDF, that is, $P\left(\omega, \sigma^{2}\right)=1$ for $\sigma_{\boldsymbol{\Delta}}^{2} \rightarrow 0$. In this case, the optimum Wiener filter in (3.44) depends mainly on the power spectral density of the residual noise according to

$$
F_{o}(\omega)=\frac{1}{N+\alpha(\omega)} \mathbf{1}^{T}
$$

If we neglect the residual noise in (3.50), i.e., $\alpha(\omega) \rightarrow 0$, we obtain the averaging filter according to (3.34). In other words, considering only signal components that capture the motion in the model, the averaging filter is, in the limit, the optimum filter for very accurate motion compensation. Note, that the complementary hypotheses are not identical, even for very accurate motion compensation.

\subsection{Forward-Adaptive Hypothesis Switching}

Superimposed motion-compensated prediction combines more than one motion-compensated signal, or hypothesis, to predict the current frame signal. In particular, we do not specify how the multiple hypotheses are selected. Now, we assume that they are determined by forward-adaptive hypothesis switching [FG01b]. Hypothesis switching selects one motion-compensated signal from a set of $M$ reference frames. The parameter which indicates a particular frame in the set of $M$ reference frames is transmitted as sideinformation to the decoder. This models multiframe motion-compensated prediction as discussed in Section 2.2.4. In the following, we discuss this concept of forward-adaptive hypothesis switching for superimposed motion-compensated prediction and its performance with complementary hypotheses.

Assume that we linearly combine $N$ hypotheses. Each hypothesis that is used for the combination is selected from a set of motion-compensated signals of size $M$. We study the influence of the hypothesis set size $M$ on both the accuracy of motion compensation of forward-adaptive hypothesis switching and the efficiency of superimposed motion estimation. In both cases, we examine the noise-free limiting case. That is, we neglect signal components that are not predictable by motion compensation. Selecting one hypothesis from a set of motion-compensated signals of size $M$, that is, switching among $M$ hypotheses, reduces the displacement error variance by factor $M$, if we assume statistically independent displacement errors. Integrating forward-adaptive hypothesis switching into superimposed motion-compensated prediction, that is, allowing a combination of switched hypotheses, increases the gain of superimposed motion-compensated prediction over the single hypothesis case for growing hypothesis set size $M$.

Experimental results in Section 4.3.3 and 5.3.2 confirm that block-based multiframe motion compensation enhances the efficiency of superimposed prediction. The experimental setup is such that we combine both block-based predictors and superimpose $N$ hypotheses where each hypothesis is obtained by switching among $M$ motion-compensated blocks. 


\subsubsection{Signal Model for Hypothesis Switching}

A signal model for hypothesis switching is depicted in Fig. 3.15 for two hypotheses. The current frame signal $\mathbf{s}[l]$ at discrete location $l=(x, y)$ is predicted by selecting between $M$ hypotheses $\mathbf{c}_{\mu}[l]$ with $\mu=1, \ldots, M$. The resulting prediction error is denoted by $\mathbf{e}[l]$.

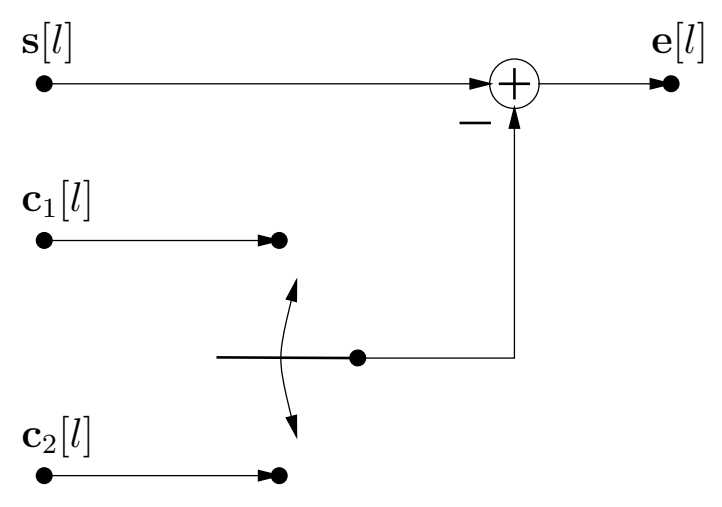

Figure 3.15: Forward-adaptive hypothesis switching for motion-compensated prediction.

To capture the limited accuracy of motion compensation, we associate a vector valued displacement error $\boldsymbol{\Delta}_{\mu}$ with the $\mu$-th hypothesis $\mathbf{c}_{\mu}$. The displacement error reflects the inaccuracy of the displacement vector used for motion compensation. We assume a 2 -D stationary normal distribution with variance $\sigma_{\Delta}^{2}$ and zero mean where $x$ - and $y$ components are statistically independent. The displacement error variance is the same for all $M$ hypotheses. This is reasonable because all hypotheses are compensated with the same accuracy. Further, the pairs $\left(\boldsymbol{\Delta}_{\mu}, \boldsymbol{\Delta}_{\nu}\right)$ are assumed to be statistically independent Gaussian random variables and that the displacement error for all hypotheses are spatially constant.

For simplicity, we assume that all hypotheses $\mathbf{c}_{\mu}$ are shifted versions of the current frame signal $\mathbf{s}$. The shift is determined by the displacement error $\boldsymbol{\Delta}_{\mu}$ of the $\mu$-th hypothesis. For that, the ideal reconstruction of the space-discrete signal $\mathbf{s}[l]$ is shifted by the continuous valued displacement error and re-sampled on the original orthogonal grid.

This model neglects "noisy" signal components and assumes that motion accuracy is basically the decision criterion for switching. As previously discussed in Section 3.2.3, prediction error variance decreases by reducing the displacement error variance of hypotheses that are used for prediction. But a smaller displacement error variance can only be achieved by increasing the probability of individual displacement error that are "close" to the origin. In other words, we select from the set of $M$ motion-compensated signals the one with the smallest displacement error.

\subsubsection{Minimizing the Radial Displacement Error}

Hypothesis switching improves the accuracy of motion compensation by selecting among $M$ hypotheses the one with the smallest displacement error. Now, let us assume that 
the components of the displacement error for each hypothesis are i.i.d. Gaussian. The Euclidean distance to the zero displacement error vector defines the radial displacement error for each hypothesis.

$$
\Delta_{\mathbf{r} \mu}=\sqrt{\Delta_{\mathbf{x} \mu}^{2}+\Delta_{\mathbf{y}_{\mu}^{2}}^{2}}
$$

We assume that the hypothesis with minimum radial displacement error

$$
\Delta_{\mathbf{r}}^{M}=\min _{\mu}\left(\Delta_{\mathbf{r} 1}, \ldots, \Delta_{\mathbf{r} \mu}, \ldots, \Delta_{\mathbf{r} M}\right)
$$

is used to predict the signal.

In the following, we describe hypothesis switching by means of the reliability function [Pap91] of the minimum radial displacement error. The reliability function $R_{\boldsymbol{\Delta}_{\mathbf{r}}{ }^{M}}(r)$ is closely related to the distribution function and is defined as the probability of the event that $\Delta_{\mathbf{r}}{ }^{M}$ is larger than $r$.

$$
R_{\boldsymbol{\Delta}_{\mathbf{r}}{ }^{M}}(r)=\operatorname{Pr}\left\{\boldsymbol{\Delta}_{\mathbf{r}}{ }^{M}>r\right\}
$$

The reliability function of the minimum radial displacement error can be expressed in terms of the reliability function of the set of $M$ hypotheses. The probability of the event that the minimum radial displacement error is larger than $r$ is equal to the probability of the event that all radial displacement errors are larger than $r$.

$$
\begin{aligned}
R_{\boldsymbol{\Delta}_{\mathbf{r}} M}(r) & =\operatorname{Pr}\left\{\min _{\mu}\left(\boldsymbol{\Delta}_{\mathbf{r} 1}, \ldots, \boldsymbol{\Delta}_{\mathbf{r} \mu}, \ldots, \boldsymbol{\Delta}_{\mathbf{r} M}\right)>r\right\} \\
& =\operatorname{Pr}\left\{\boldsymbol{\Delta}_{\mathbf{r} 1}>r, \ldots, \boldsymbol{\Delta}_{\mathbf{r} M}>r\right\} \\
& =R_{\boldsymbol{\Delta}_{\mathbf{r} 1 \ldots \Delta_{\mathbf{r} M}}}(r, \ldots, r)
\end{aligned}
$$

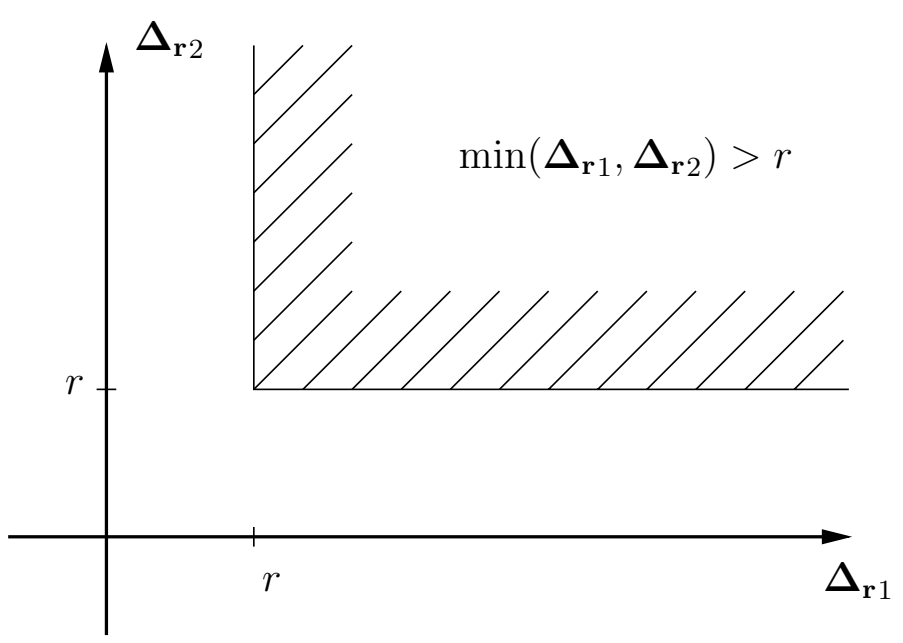

Figure 3.16: The area in which the minimum of $\boldsymbol{\Delta}_{\mathrm{r} 1}$ and $\boldsymbol{\Delta}_{\mathrm{r} 2}$ is larger than $r$.

Fig. 3.16 depicts an example for switching two hypotheses. It marks the area in which the minimum of two radial displacement errors is larger than $r$. Consequently, the probability of the event that the minimum radial displacement error is larger than $r$ is equal to the probability of the event that both radial displacement errors are larger than $r$. 
Each displacement error is drawn from a 2-D normal distribution with zero mean and variance $\sigma_{\boldsymbol{\Delta}_{\mu}}^{2}$. The displacement errors of the $M$ hypotheses are assumed to be statistically independent. The $x$ - and $y$-components of the displacement errors are arranged to vectors $\boldsymbol{\Delta}_{\mathbf{x}}$ and $\boldsymbol{\Delta}_{\mathbf{y}}$, respectively.

$$
\begin{gathered}
p_{\boldsymbol{\Delta}_{\mathbf{x}} \boldsymbol{\Delta}_{\mathbf{y}}}\left(\Delta_{x}, \Delta_{y}\right)=\frac{1}{(2 \pi)^{M} \operatorname{det}\left(C_{\boldsymbol{\Delta}_{\mathbf{x}} \boldsymbol{\Delta}_{\mathbf{x}}}\right)} e^{-\frac{1}{2}\left[\Delta_{x}^{T} C_{\boldsymbol{\Delta}_{\mathbf{x}} \boldsymbol{\Delta}_{\mathbf{x}}}^{-1} \Delta_{x}+\Delta_{y}^{T} C_{\boldsymbol{\Delta}_{\mathbf{x}} \boldsymbol{\Delta}_{\mathbf{x}}}^{-1} \Delta_{y}\right]} \\
C_{\boldsymbol{\Delta}_{\mathbf{x}} \boldsymbol{\Delta}_{\mathbf{x}}}=C_{\boldsymbol{\Delta}_{\mathbf{y}} \boldsymbol{\Delta}_{\mathbf{y}}}=\left(\begin{array}{cccc}
\sigma_{\boldsymbol{\Delta}_{\mathbf{x} 1}}^{2} & 0 & \cdots & 0 \\
0 & \sigma_{\boldsymbol{\Delta}_{\mathbf{x} 2}}^{2} & \cdots & 0 \\
\vdots & \vdots & \ddots & \vdots \\
0 & 0 & \cdots & \sigma_{\boldsymbol{\Delta}_{\mathbf{x} M}}^{2}
\end{array}\right)
\end{gathered}
$$

The criterion for switching is the radial displacement error. To obtain a closed-form expression of the radial displacement error PDF, it is assumed that the variances in $x$ and $y$-direction are identical. This is reasonable if the accuracy of motion compensation is identical for both dimensions. With this assumption, we can easily determine the probability density function of $\boldsymbol{\Delta}_{\mathbf{r}}$ [Pap91]:

$$
p_{\boldsymbol{\Delta}_{\mathbf{r}}}\left(\Delta_{r}\right)=\left(\prod_{\mu=1}^{M} \Delta_{r \mu}\right) \frac{1}{\operatorname{det}\left(C_{\boldsymbol{\Delta}_{\mathbf{x}} \boldsymbol{\Delta}_{\mathbf{x}}}\right)} e^{-\frac{1}{2} \Delta_{r}^{T} C_{\boldsymbol{\Delta}_{\mathbf{x}} \boldsymbol{\Delta}_{\mathbf{x}}}^{-1} \Delta_{r}}
$$

An $M$-dimensional Rayleigh PDF is obtained describing $M$ independent radial displacement errors.

In order to minimize the radial displacement error, the $M$-dimensional reliability function of the displacement error has to be determined.

$$
\begin{aligned}
R_{\Delta_{\mathbf{r} 1} \ldots \Delta_{\mathbf{r} M}}\left(\Delta_{r 1} \ldots \Delta_{r M}\right) & =\int_{\Delta_{r 1}}^{\infty} \ldots \int_{\Delta_{r M}}^{\infty} p_{\Delta_{\mathbf{r}}}(u) d u \\
& =e^{-\frac{1}{2} \Delta_{r}^{T} C_{\Delta_{\mathbf{x}} \Delta_{\mathbf{x}}}^{-1} \Delta_{r}}
\end{aligned}
$$

The reliability function of the minimum radial displacement error $R_{\boldsymbol{\Delta}_{\mathbf{r}}{ }^{M}}(r)$ is obtained by evaluating the $M$-dimensional reliability function at the same value $r$ for all dimensions: $R_{\boldsymbol{\Delta}_{\mathbf{r}}{ }^{M}}(r)=R_{\boldsymbol{\Delta}_{\mathbf{r} 1 \ldots \boldsymbol{\Delta}_{\mathbf{r} M}}}(\mathbf{1} r)$. $\mathbf{1}$ denotes the vector with all components equal to one. The minimum radial displacement error is also Rayleigh distributed. Note that a one-dimensional PDF is given by the negative derivative of the reliability function.

$$
\begin{aligned}
R_{\mathbf{\Delta}_{\mathbf{r}} M}(r) & =e^{-\frac{1}{2} \frac{r^{2}}{\tau^{2}}} \\
p_{\boldsymbol{\Delta}_{\mathbf{r}} M}(r) & =\frac{r}{\tau^{2}} e^{-\frac{1}{2} \frac{r^{2}}{\tau^{2}}} \\
\text { with } \tau^{2} & =\frac{1}{\mathbf{1}^{T} C_{\boldsymbol{\Delta}_{\mathbf{x}} \boldsymbol{\Delta}_{\mathbf{x}}}^{-1}}
\end{aligned}
$$

The variance of the minimum radial displacement error is of interest. The covariance matrix of the Rayleigh PDF in (3.57) is $C_{\boldsymbol{\Delta}_{\mathbf{r}} \boldsymbol{\Delta}_{\mathbf{r}}}=\left(2-\frac{\pi}{2}\right) C_{\boldsymbol{\Delta}_{\mathbf{x}} \boldsymbol{\Delta}_{\mathbf{x}}}$ and the variance of the 
switched radial displacement error is given by $\sigma_{\boldsymbol{\Delta}_{\mathbf{r}}{ }^{M}}^{2}=\left(2-\frac{\pi}{2}\right) \tau^{2}$ [LG94]. In order to omit the constant factor, the variance of the minimum radial displacement error is stated as a function of the covariance matrix $C_{\boldsymbol{\Delta}_{\mathrm{r}} \boldsymbol{\Delta}_{\mathrm{r}}}$.

$$
\sigma_{\boldsymbol{\Delta}_{\mathbf{r}} M}^{2}=\frac{1}{\mathbf{1}^{T} C_{\boldsymbol{\Delta}_{\mathbf{r}} \mathbf{\Delta}_{\mathbf{r}}}^{-1} \mathbf{1}}
$$

For example, the variances of the radial displacement errors might be identical for all $M$ hypotheses. (3.62) implies that switching of independent Rayleigh distributed radial displacement errors reduces the variance by factor $M$.

$$
\sigma_{\Delta_{\mathrm{r}} M}^{2}=\frac{\sigma_{\Delta_{\mathrm{r}}}^{2}}{M}
$$

\subsubsection{Equivalent Predictor}

Section 3.4.2 shows that both the individual radial displacement errors and the minimum radial displacement error are Rayleigh distributed. This suggests to define an equivalent motion-compensated predictor for switched prediction that uses just one hypothesis but with a much smaller the displacement error variance. The equivalent distribution of the displacement error is assumed to be separable and normal with zero mean and variance

$$
\sigma_{\boldsymbol{\Delta}_{\mathbf{x}}{ }^{M}}^{2}=\frac{1}{\mathbf{1}^{T} C_{\boldsymbol{\Delta}_{\mathbf{x}} \boldsymbol{\Delta}_{\mathbf{x}}}^{-1} \mathbf{1}}
$$

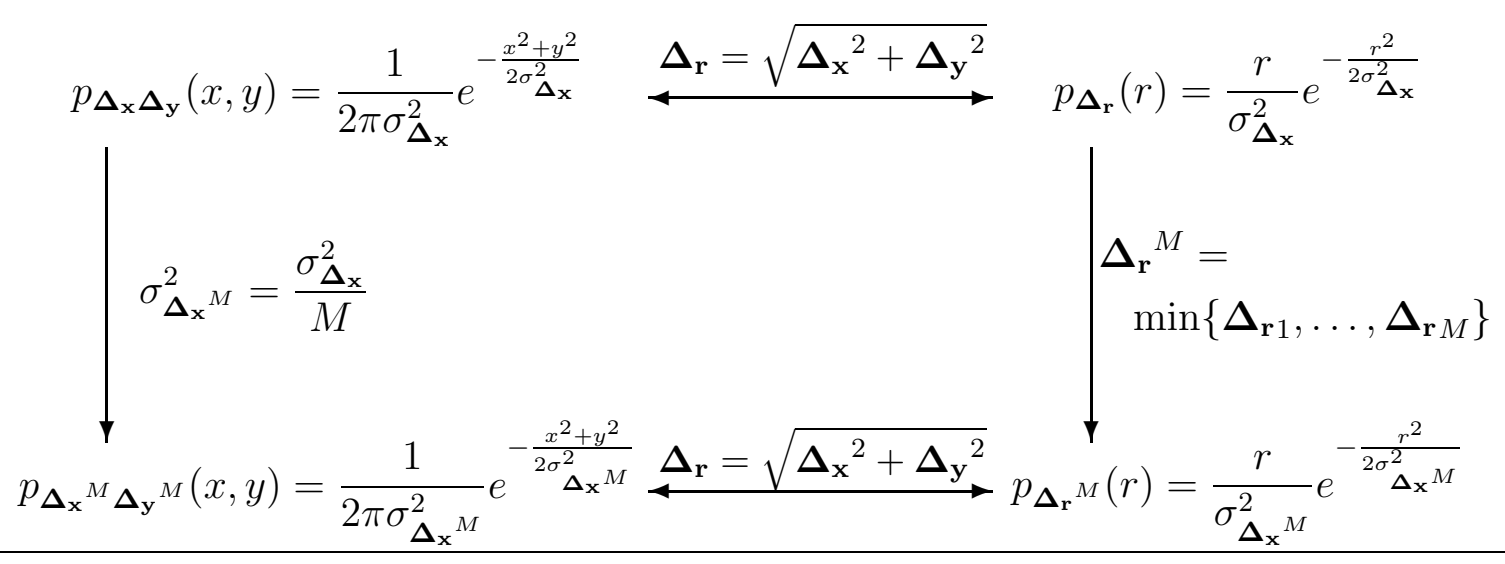

Figure 3.17: The probability density functions of Cartesian and radial displacement errors for switching $M$ independent and identically distributed displacement error.

Figure 3.17 gives an example for the equivalent predictor when switching $M$ independent and identically distributed displacement error signals. The 2-D and Gaussian distributed displacement error of each hypothesis is transformed into the Rayleigh distributed radial displacement error. Minimizing $M$ independent radial displacement error signals yields a radial displacement error which is also Rayleigh distributed. The inverse 
transform into the 2-D Cartesian coordinate system determines the 2-D normal distributed displacement error of the equivalent predictor. Its displacement error variance is reduced by factor $M$ in comparison to the variance of individual hypotheses.

The equivalent predictor suggests a model for switched prediction with just one hypothesis but reduced displacement error variance. This reduced variance determines the accuracy of motion-compensated prediction with forward-adaptive hypothesis switching. That is, switching improves the accuracy of motion-compensated prediction by reducing the displacement error variance.

\subsubsection{Motion Compensation with Complementary Hypotheses and Forward-Adaptive Hypothesis Switching}

We combine forward-adaptive hypothesis switching with complementary hypotheses motion-compensated prediction such that we superimpose $N$ complementary hypotheses where each hypothesis is obtained by switching among $M$ motion-compensated signals.

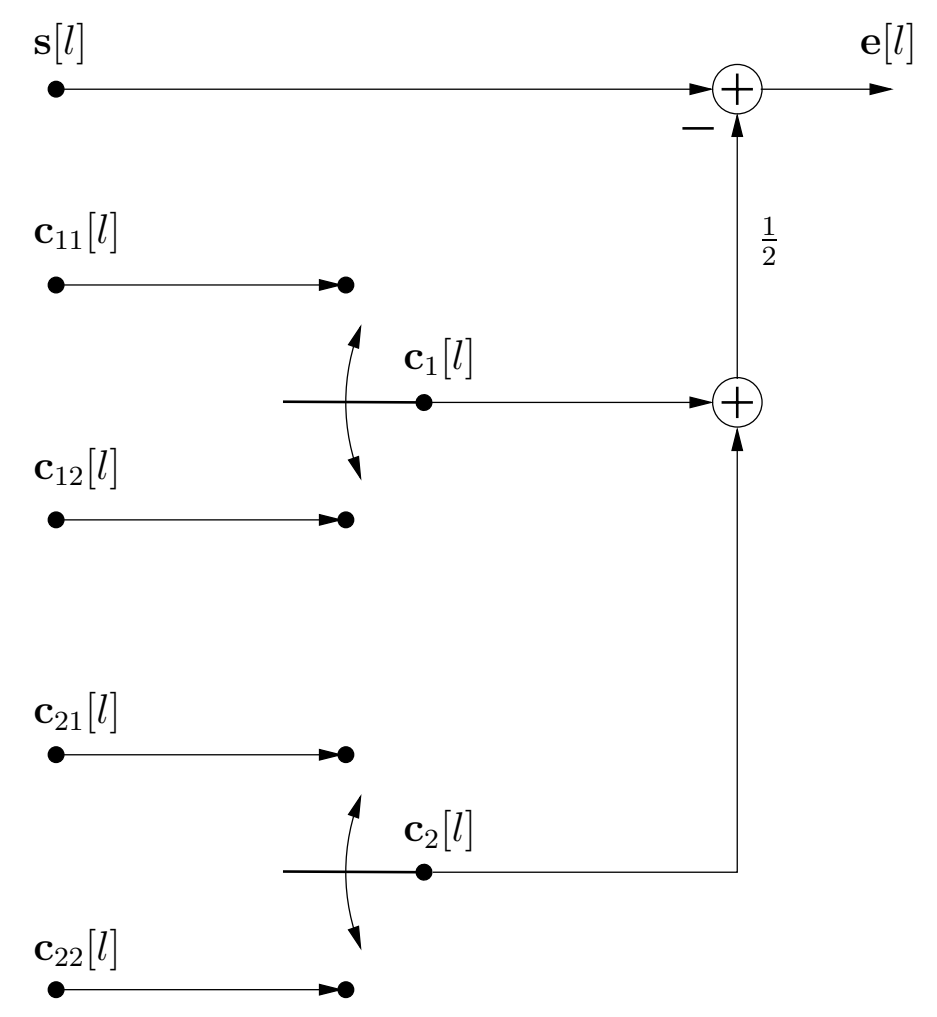

Figure 3.18: Motion-compensated prediction with $N=2$ complementary hypotheses and forward-adaptive hypothesis switching $M=2$.

Fig. 3.18 depicts a prediction scheme where the complementary hypotheses $\mathbf{c}_{1}$ and $\mathbf{c}_{2}$ are determined by switching for each hypothesis among $M=2$ motion-compensated signals. In practice, $N=2$ complementary hypotheses are selected from $M=2$ refer- 
ence frames. Further, as the complementary hypotheses are jointly estimated, the frame selection is also performed jointly.

Let us consider the example that we switch among $M$ motion-compensated signals and utilize complementary hypotheses motion compensation with $N=2$ hypotheses $\mathbf{c}_{1}$ and $\mathbf{c}_{2}$. Then, complementary hypotheses motion compensation uses two sets of motioncompensated signals of size $M$. The hypothesis $\mathbf{c}_{1}$ is selected from the set $\left\{\mathbf{c}_{11}, \ldots, \mathbf{c}_{1 M}\right\}$, and the complementary hypothesis $\mathbf{c}_{2}$ from the complementary set $\left\{\mathbf{c}_{21}, \ldots, \mathbf{c}_{2 M}\right\}$. Choosing one motion-compensated signal from each set provides two hypotheses whose displacement error correlation coefficient is -1 . But choosing two motion-compensated signals from the same set provides two signals whose displacement error correlation coefficient is 0 . For this example, we assume that these hypotheses exist and that an ideal superimposed motion estimator is able to determine the desired signals.

According to the previous section, choosing among $M$ motion-compensated signals can reduce the displacement error variance by up to a factor of $M$. Motion compensation with complementary hypotheses utilizes for each hypothesis forward-adaptive switching. Consequently, the displacement error variance of superimposed hypotheses $\sigma_{\Delta}^{2}$ in (3.9) is smaller by a factor of $M$

$$
\frac{\Phi_{\mathbf{e e}}(\omega)}{\Phi_{\mathbf{s s}}(\omega)}=\frac{N+1}{N}-2 P\left(\omega, \frac{\sigma_{\Delta}^{2}}{M}\right)+\frac{N-1}{N} P\left(\omega, 2 \frac{\sigma_{\Delta}^{2}}{M}\left(1-\rho_{\boldsymbol{\Delta}}\right)\right) .
$$

Note, that (3.65) represents a performance bound given the previous assumptions.

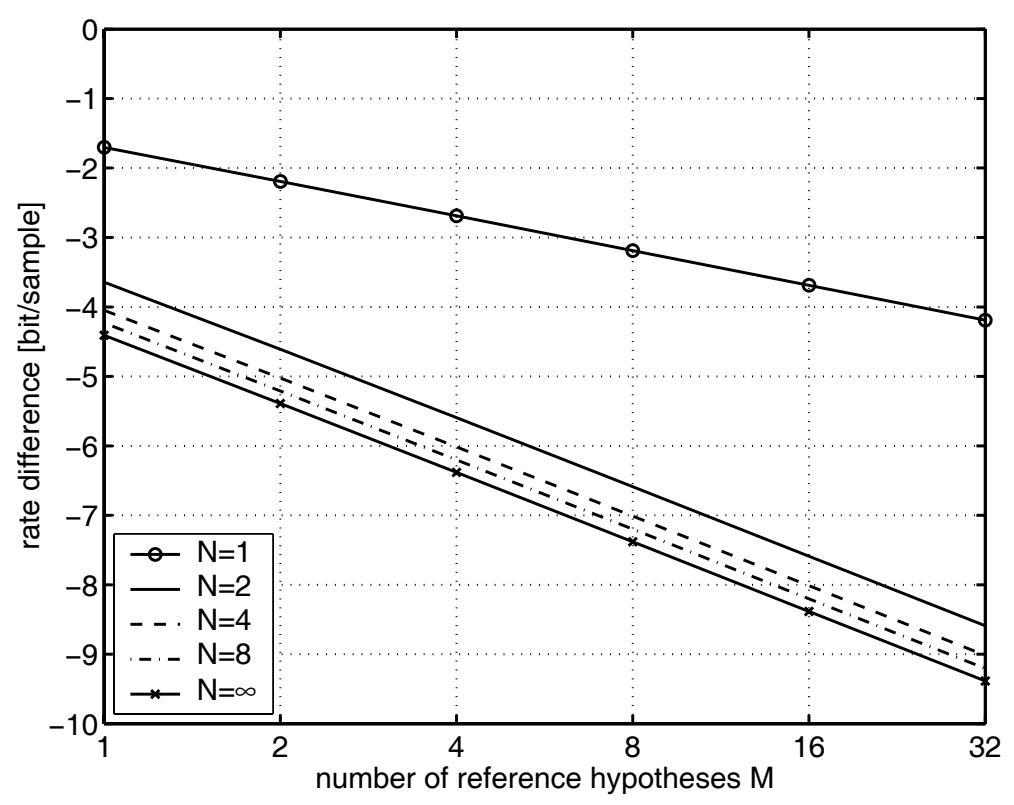

Figure 3.19: Rate difference over the number of motion-compensated signals $M$ for motion compensation with complementary hypotheses and forward-adaptive hypothesis switching. The switched hypotheses are just averaged and no residual noise is assumed. The results are for half-pel accurate motion compensation, i.e., $\beta=-1$. 
Fig. 3.19 depicts the rate difference over the size of the motion-compensated signal set $M$ according to (3.65). The performance bound of motion compensation with complementary hypotheses and forward-adaptive hypothesis switching for $N=2,4,8$, and $\infty$ linearly combined hypotheses is compared to motion-compensated prediction with forward-adaptive hypothesis switching $(N=1)$. Half-pel accurate motion compensation $(\beta=-1)$ is assumed. We observe that doubling the number of reference hypotheses decreases the bit-rate for motion-compensated prediction by 0.5 bits per sample and for motion-compensated prediction with complementary hypotheses by 1 bit per sample. Due to the different slopes, the bit-rate savings by complementary hypotheses motion compensation over single hypothesis motion compensation increase with the number of available motion-compensated signals $M$. This theoretical finding supports the experimental results in Section 4.3.3 and 5.3.2.

The experimental results show also a saturation of the gain by forward-adaptive hypothesis switching. Choosing among $M$ motion-compensated signals can reduce the displacement error variance by up to a factor of $M$. This lower bound is obtained when switching among $M$ motion-compensated signals with statistically independent displacement error. Correlated displacement error degrade the performance of hypothesis switching and cause a saturation of the bit-rate savings for an increasing number of motioncompensated signals $M$.

\subsection{Pictures with Varying Number of Hypotheses}

In practice, video sequences are usually coded with two different picture types: P- and B-pictures. P-pictures use block-based (multiframe) motion-compensated prediction with temporally prior reference pictures whereas B-pictures utilize block-based bidirectional prediction with one (multiple) temporally subsequent and one (multiple) temporally prior reference picture(s). Picture type sequences with a varying number of inserted B-pictures like $\mathrm{P}, \mathrm{PB}, \mathrm{PBB}$, and $\mathrm{PBBB}$ are widely used. Bidirectional prediction in B-pictures is a special case of superimposed motion-compensated prediction with $N=2$ hypotheses. If we neglect OBMC, motion-compensated prediction in P-pictures is predominantly single hypothesis prediction.

In the following, we investigate the impact of motion compensation with complementary hypotheses on the sequence coding performance based on the previously discussed high-rate approximation for sequential encoding. At high rates, the residual encoder guarantees infinitesimal small reconstruction distortion and any picture coding order that exploits all statistical dependencies provides the same coding efficiency. B-pictures with complementary hypothesis motion compensation provide improved prediction performance over P-pictures and their relative occurrence improves sequence coding performance. Because of the high-rate approximation, we neglect the rate of the side-information.

The average rate difference for predictive sequence coding compared to optimum intraframe sequence coding yields

$$
\Delta R=\frac{n_{P}}{n_{P}+n_{B}} \Delta R_{P}+\frac{n_{B}}{n_{P}+n_{B}} \Delta R_{B}
$$


where $n_{P}$ and $n_{B}$ are the number of $\mathrm{P}$ - and B-pictures in the sequence. $\Delta R_{P}$ denotes the rate difference of single hypothesis prediction and $\Delta R_{B}$ that of motion compensation with complementary hypotheses. The relative number of $\mathrm{P}$ - and $\mathrm{B}$-pictures in a sequence determines the contribution of a particular picture type to the average rate difference.

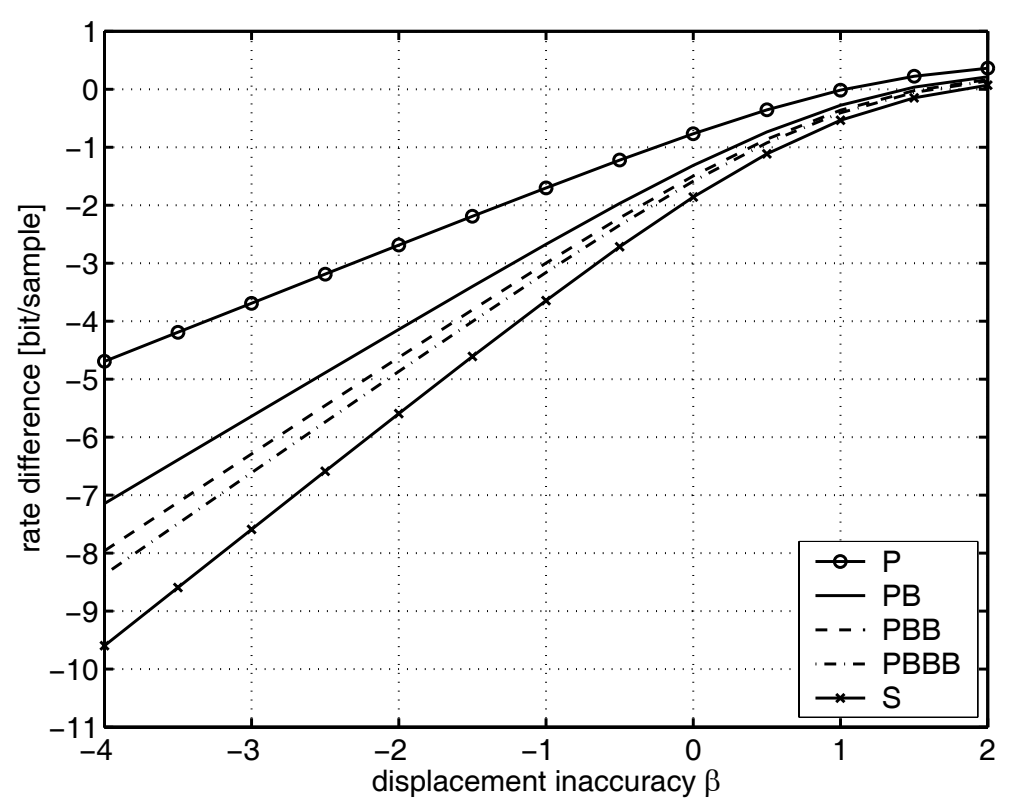

Figure 3.20: Performance bounds for five different picture type sequences. The rate difference is depicted over the displacement inaccuracy. B-pictures average two complementary hypotheses. No residual noise is assumed.

Fig. 3.20 depicts the rate difference for predictive sequence coding compared to optimum intra-frame sequence coding over the motion accuracy $\beta$. No residual noise is assumed. The rate difference is given for five different picture type sequences: P, PB, PBB, and $\mathrm{PBBB}$ with $0,6,8$, and 9 B-pictures in a group of 12 pictures where the B-pictures utilize motion-compensated prediction with two complementary hypotheses. S denotes a sequence of superimposed pictures which also use motion-compensated prediction with two complementary hypotheses. As the slope of the rate difference is not equal for Pand B-pictures, the linear combination in (3.66) affects the slope of the average rate difference. In the limit, the picture type sequences $\mathrm{PB}, \mathrm{PBB}$, and $\mathrm{PBBB}$ modify the slope of the average rate difference to $3 / 2,5 / 3$, and $7 / 4$ bits per sample per inaccuracy step, respectively.

Fig. 3.21 depicts similar to Fig. 3.20 the rate difference for predictive sequence coding compared to optimum intra-frame sequence coding over the motion accuracy $\beta$ but with a residual noise of $\mathrm{RNL}=-30 \mathrm{~dB}$. In the presence of residual noise, the advantage due to the slope difference is mainly prevalent in the range between quarter-pel and integer-pel accuracy. Beyond quarter-pel accuracy, the residual noise dominates and the average rate difference starts to saturate. 


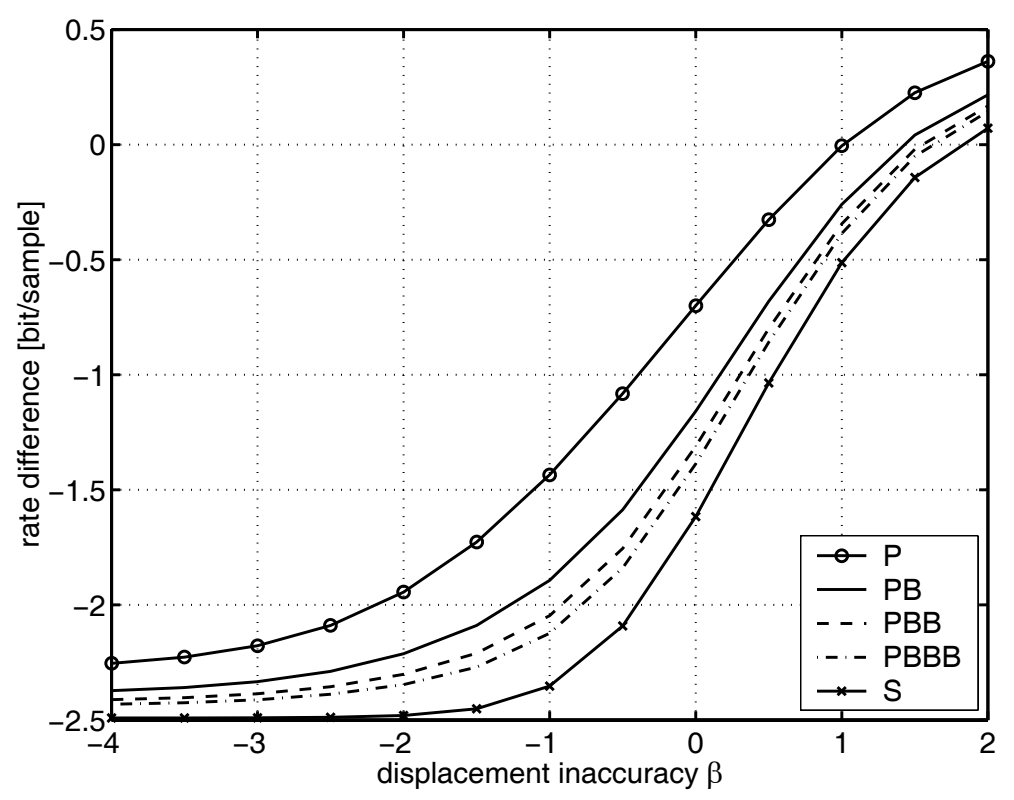

Figure 3.21: Performance bounds for five different picture type sequences. The rate difference is depicted over the displacement inaccuracy. B-pictures average two complementary hypotheses. The residual noise is $-30 \mathrm{~dB}$.

\subsection{Conclusions}

This chapter extends the theory of multihypothesis motion-compensated prediction by introducing the concept of motion-compensation with complementary hypotheses. We allow for the displacement errors of $N$ hypotheses to be correlated. The assumption that the $N$ displacement errors are jointly distributed imposes an constraint on the displacement error correlation coefficient as any covariance matrix is nonnegative definite. We analyze the dependency between the displacement error correlation coefficient and the performance of superimposed motion compensation. A ideal superimposed motion estimator minimizes the prediction error and, consequently, the displacement error correlation coefficient. The optimal ideal superimposed motion estimator is the estimator that determines $N$ complementary hypotheses with maximally negatively correlated displacement error. This is a result which is independent of the underlying frame signal model.

Assuming band-limited and noise-free frame signals, the motion compensator with complementary hypotheses achieves a rate difference of up to 2 bits per sample per inaccuracy step whereas a single hypothesis motion compensator is limited to 1 bit per sample per inaccuracy step. When averaging more than two hypotheses, the bit-rate savings are limited even if the number of hypotheses grows very large. When utilizing the optimum Wiener filter, the bit-rate savings are not limited and doubling the number of hypotheses improves the rate difference by 0.5 bits per sample for a very large number of hypotheses.

In the presence of residual noise, the bit-rate savings saturate for increasing motion accuracy. The optimum Wiener filter with an infinite number of hypotheses permits bitrate savings that are independent of the motion accuracy and limited only by the residual 
noise. In addition, the Wiener filter for motion compensation with complementary hypotheses amplifies high frequency components and shows band-pass characteristics. The optimum Wiener filter for single hypothesis motion compensation shows only low-pass characteristics.

This chapter also combines forward-adaptive hypothesis switching with complementary hypothesis motion compensation. Choosing among $M$ motion-compensated signals with statistically independent displacement error reduces the displacement error variance by up to a factor of $M$. We utilize motion compensation with complementary hypotheses such that each hypothesis is determined by switching among $M$ reference hypotheses. An analysis of the noise-free case shows that doubling the number of reference hypotheses decreases the bit-rate of motion-compensated prediction by 0.5 bits per sample and that of motion compensation with complementary hypotheses by 1 bit per sample. Due to the different slopes, the bit-rate savings by complementary hypotheses motion compensation over single hypothesis motion compensation increase with the number of available reference signals $M$.

Finally, this chapter discusses sequence coding with different picture types characterized by a varying number of hypotheses. As the slope of the rate difference is not equal for single hypothesis prediction (P-pictures) and superimposed prediction with complementary hypotheses (B-pictures), particular picture type sequences influence the slope of the overall average rate difference. The analysis suggests that the number of pictures with complementary hypotheses motion compensation should be increased such that the overall average rate difference benefits from complementary hypotheses. 


\section{ITU-T Rec. H.263 and Superimposed Prediction}

\subsection{Introduction}

This chapter discusses a practical implementation of superimposed motion-compensated prediction according to Section 2.2.5, based on the ITU-T Recommendation H.263 [ITU98a, ITU00]. The general concept of superimposed prediction with multiple reference frames is not part of this standard. We investigate the efficient number of hypotheses, the combination with variable block size prediction, and the influence of multiframe motion compensation. Further, we relate the experimental results to the insights from Chapter 3.

ITU-T Recommendation H.263 utilizes a hybrid video coding concept with blockbased motion-compensated prediction and DCT-based transform coding of the prediction error. P-frame coding of H.263 employs INTRA and INTER coding modes. Superimposed motion-compensated prediction for P-frame coding is enabled by new coding modes that are derived from H.263 INTER coding modes. Annex U of ITU-T Rec. H.263 allows multiframe motion-compensated prediction but does not provide capabilities for superimposed prediction. A combination of H.263 Annex U with B-frames leads to the concept of superimposed multiframe prediction. In this chapter, we do not use H.263 B-frames as we discuss interpolative prediction for in-order encoding of sequences. H.263 B-frames can only be used for out-of-order encoding of sequences. Further, the presented concept of superimposed multiframe prediction is much more general than the B-frames in H.263. ITU-T Rec. H.263 also provides OBMC capability. As discussed previously, OBMC uses more than one motion vector for predicting the same pixel but those motion vectors are also used by neighboring blocks. In this chapter, a block predicted by superimposed motion compensation has its individual set of motion vectors. We do not overlap shifted blocks that might be obtained by utilizing spatially neighboring motion vectors. The INTER4V coding mode of H.263 utilizes VBS prediction with either OBMC or an in-loop deblocking filter. Superimposed prediction already filters the motion-compensated signals and an extension of H.263 OBMC or in-loop deblocking filter is not implemented.

The outline of this chapter is as follows: Section 4.2 explains the video codec with superimposed motion-compensated prediction. We outline syntax extensions for H.263 as well as coder control issues for mode decision and motion estimation. Section 4.3 discusses several experiments. We investigate the efficient number of hypotheses, combine superimposed prediction with blocks of variable size, and study the influence of multiframe motion compensation. 


\subsection{Video Coding with Superimposed Motion}

ITU-T Recommendation H.263 standardizes a block-based hybrid video codec. Such a codec utilizes motion-compensated prediction to generate a prediction signal from previous reconstructed frames in order to reduce the bit-rate of the residual encoder. For blockbased MCP, one motion vector and one picture reference parameter which address the reference block in a previous reconstructed frame are assigned to each block in the current frame.

The superposition video codec [FWG00b] additionally reduces the bit-rate of the residual encoder by improving the prediction signal. The improvement is achieved by combining linearly more than one motion-compensated prediction signal. For block-based superimposed MCP, more than one motion vector and picture reference parameter, which address a reference block in previous reconstructed frames, is assigned to each block in the current frame. These multiple reference blocks are linearly combined to form the block-based superimposed prediction signal.

The coding efficiency is improved at the expense of increased computational complexity for motion estimation at the encoder. But this disadvantage can be tackled by efficient estimation strategies like successive elimination as discussed in Section 2.3.4. At the decoder, a minor complexity increase is caused by the selection and combination of multiple prediction signals. Please note that not all macroblocks utilize superimposed MCP.

\subsubsection{Syntax Extensions}

The syntax of H.263 is extended such that superimposed motion compensation is possible. On the macroblock level, two new modes, INTER2H and INTER4H, are added which allow two or four hypotheses per macroblock, respectively. These modes are similar to the INTER mode of H.263. The INTER2H mode additionally includes an extra motion vector and frame reference parameter for the second hypothesis. The INTER4H mode incorporates three extra motion vectors and frame reference parameters. For variable block size prediction, the INTER4V mode of H.263 is extended by a superposition block pattern. This pattern indicates for each $8 \times 8$ block the number of motion vectors and frame reference parameters. This mode is called INTER4VMH. The superposition block pattern has the advantage that the number of hypotheses can be indicated individually for each $8 \times 8$ block. This allows the important case that just one $8 \times 8$ block can be coded with more than one motion vector and frame reference parameter. The INTER4VMH mode includes the INTER4V mode when the superposition block pattern indicates just one hypothesis for all $8 \times 8$ blocks.

\subsubsection{Coder Control}

The coder control for the superposition video codec utilizes rate-distortion optimization by Lagrangian methods. For that, the average Lagrangian cost of a macroblock, given the 
previously encoded macroblocks, is minimized. The average cost $J=D+\lambda R$ consists of the average distortion $D$ and the weighted average bit-rate $R$. The weight, also called Lagrange multiplier $\lambda$, is related to the macroblock quantization parameter $Q P$ by the relationship

$$
\lambda=0.85 Q P^{2}
$$

as discussed in Section 2.3.3. This generic optimization method provides the encoding strategy for the superposition encoder: Minimizing the instantaneous Lagrangian costs for each macroblock minimizes the average Lagrangian costs, given the previous encoded macroblocks.

H.263 allows several encoding modes for each macroblock. The one with the lowest Lagrangian costs is selected for the encoding. This strategy is also called rate-constrained mode decision $\left[\mathrm{WLM}^{+}\right.$96], [SW98].

The new superposition modes include both superimposed prediction and prediction error encoding. The Lagrangian costs of the new superposition modes have to be evaluated for rate-constrained mode decision. The distortion of the reconstructed macroblock is determined by the summed squared error. The macroblock bit-rate includes also the rate of all motion vectors and picture reference parameters. This allows the best trade-off between superimposed MCP rate and prediction error rate [Gir94].

As already mentioned, superimposed MCP improves the prediction signal by spending more bits for the side-information associated with the motion-compensating predictor. But the encoding of the prediction error and its associated bit-rate also determines the quality of the reconstructed block. A joint optimization of superimposed motion estimation and prediction error encoding is far too demanding. But superimposed motion estimation independent of prediction error encoding is an efficient and practical solution. This solution is efficient if rate-constrained superimposed motion estimation, as explained before, is applied.

For example, the encoding strategies for the INTER and INTER2H modes are as follows: Testing the INTER mode, the encoder performs successively rate-constrained motion estimation for integer-pel positions and rate-constrained half-pel refinement. Rateconstrained motion estimation incorporates the prediction error of the video signal as well as the bit-rate for the motion vector and picture reference parameter. Testing the INTER2H mode, the encoder performs rate-constrained superimposed motion estimation. Rate-constrained superimposed motion estimation incorporates the superimposed prediction error of the video signal as well as the bit-rate for two motion vectors and picture reference parameters. Rate-constrained superimposed motion estimation is performed by the HSA in Fig. 2.6 which utilizes in each iteration step rate-constrained motion estimation to determine a conditional rate-constrained motion estimate. Given the obtained motion vectors and picture reference parameters for the INTER and INTER2H modes, the resulting prediction errors are encoded to evaluate the mode costs. The encoding strategy for the INTER4H mode is similar. For the INTER4VMH mode, the number of hypotheses for each $8 \times 8$ block is determined after encoding its residual error. 


\subsection{Experimental Results}

The superposition codec is based on the ITU-T Rec. H.263 with unrestricted motion vector mode, four motion vectors per macroblock, and enhanced reference picture selection in sliding window buffering mode. In contrast to H.263, the superposition codec uses a joint entropy code for horizontal and vertical motion vector data as well as an entropy code for the picture reference parameter. The efficiency of the reference codec is comparable to those of the H.263 test model TMN-10 [ITU98b]. The test sequences are coded at QCIF resolution and $10 \mathrm{fps}$. Each sequence has a length of ten seconds. For comparison purposes, the PSNR values of the luminance component are measured and plotted over the total bit-rate for the quantization parameter $4,5,7,10,15$, and 25 . The data of the first intra-frame coded picture, which is identical in all cases, is excluded from the results.

\subsubsection{Multiple Hypotheses for Constant Block Size}

We investigate the coding efficiency of superimposed prediction with two and four hypotheses for constant block size. Figs. 4.1 and 4.2 depict the average luminance PSNR from reconstructed frames over the overall bit-rate for the sequences Foreman and Mobile $\&$ Calendar, respectively. The performance of the codec with baseline prediction (BL), superimposed prediction with two hypotheses (BL+INTER2H), and four hypotheses $(\mathrm{BL}+\mathrm{INTER} 2 \mathrm{H}+\mathrm{INTER} 4 \mathrm{H})$ is shown. In each case, $M=10$ reference pictures are utilized for prediction. The baseline performance for single frame prediction $(M=1)$ is added for reference.

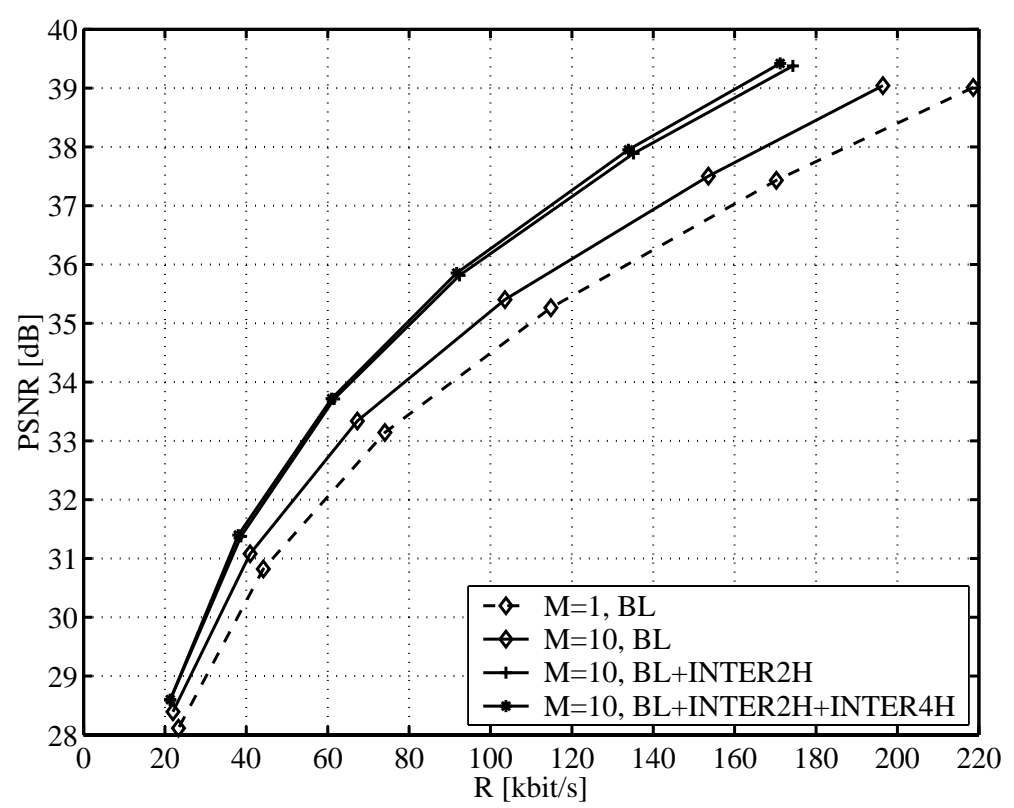

Figure 4.1: Average luminance PSNR over total rate for the sequence Foreman depicting the performance of the superposition coding scheme for constant block size. $M=10$ reference pictures are utilized for prediction. 


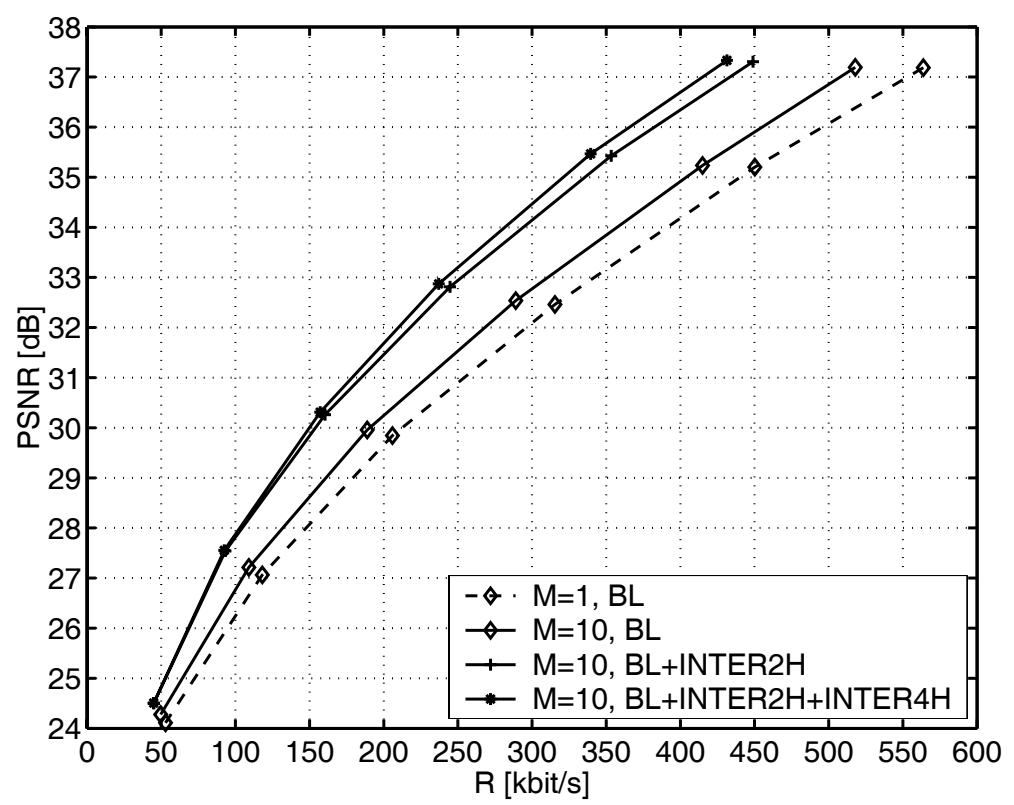

Figure 4.2: Average luminance PSNR over total rate for the sequence Mobile \& Calendar depicting the performance of the superposition coding scheme for constant block size. $M=10$ reference pictures are utilized for prediction.

Superimposed prediction is enabled by allowing the INTER2H mode on the macroblock level. A gain of up to $1 \mathrm{~dB}$ for the sequence Foreman and $1.4 \mathrm{~dB}$ for the sequence Mobile $\&$ Calendar is achieved by the INTER2H mode. Superimposed prediction with up to four hypotheses is implemented such that each predictor type (depending on the number of superimposed signals) constitutes a coding mode. A rate-distortion efficient codec should utilize four hypotheses only when their coding gain is justified by the associated bit-rate. In the case that four hypotheses are not efficient, the codec should be able to select two hypotheses and choose the INTER2H mode. The additional INTER4H mode gains just up to $0.1 \mathrm{~dB}$ for the sequence Foreman and $0.3 \mathrm{~dB}$ for the sequence Mobile $\&$ Calendar. This results support the finding in Section 3.2.2 that two hypotheses provide the largest relative gain. Considering both this insight and the computational complexity for estimating four hypotheses, we will restrict the superposition coding scheme to two hypotheses.

Finally, we consider hypotheses that are not optimized with the hypothesis selection algorithm in Fig. 2.6. With only previous reference frames, not optimized hypotheses are as good as or worse (due to the bit-rate of additional motion vectors) than single hypothesis prediction. In that case, the mode selection prefers the single hypothesis mode and the rate-distortion performance is identical to that with the label " $M=10, \mathrm{BL}$ ".

\subsubsection{Multiple Hypotheses for Variable Block Size}

In this subsection, we investigate the influence of variable block size (VBS) prediction on superimposed prediction for $M=10$ reference pictures. VBS prediction in H.263 
is enabled by the INTER4V mode which utilizes four motion vectors per macroblock. Both VBS prediction and superimposed prediction use more than one motion vector per macroblock which is transmitted to the decoder as side-information. But both concepts provide gains for different scenarios. This can be verified by applying superimposed prediction to blocks of size $16 \times 16$ (INTER2H) as well as $8 \times 8$ (INTER4VMH). As we permit a maximum of two hypotheses per block, one bit is sufficient to signal whether one or two prediction signals are used.

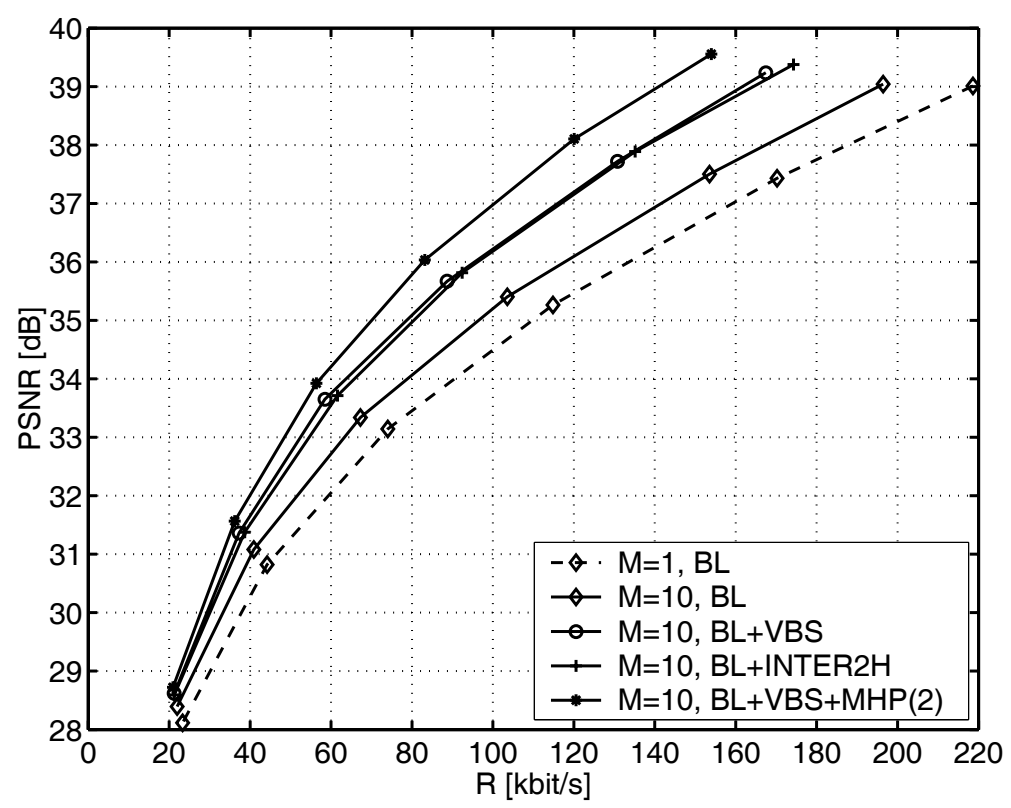

Figure 4.3: Average luminance PSNR over total rate for the sequence Foreman. Superimposed and variable block size prediction can be successfully combined for compression. $M=10$ reference pictures are utilized for prediction.

Figs. 4.3 and 4.4 depict the average luminance PSNR from reconstructed frames over the overall bit-rate for the sequences Foreman and Mobile $\&$ Calendar. The performance of the codec with baseline prediction (BL), VBS prediction (BL+VBS), superimposed prediction with two hypotheses (BL+INTER2H), and superimposed prediction with variable block size $(\mathrm{BL}+\mathrm{VBS}+\mathrm{MHP}(2))$ is shown. In each case, $M=10$ reference pictures are utilized for prediction. The baseline performance for single frame prediction $(M=1)$ is added for reference.

The combination of superimposed and variable block size prediction yields superior compression efficiency. For example, to achieve a reconstruction quality of $35 \mathrm{~dB}$ in PSNR, the sequence Mobile $\&$ Calendar is coded in baseline mode with $403 \mathrm{kbit} / \mathrm{s}$ for $M=10$ (See Fig. 4.4). Correspondingly, superimposed prediction with $M=10$ reduces the bitrate to $334 \mathrm{kbit} / \mathrm{s}$. Superimposed prediction on macroblocks decreases the bit-rate by $17 \%$. Performing superimposed prediction additionally on $8 \times 8$ blocks, the bit-rate is $290 \mathrm{kbit} / \mathrm{s}$ in contrast to $358 \mathrm{kbit} / \mathrm{s}$ for the codec with VBS. Superimposed prediction decreases the bit-rate by $19 \%$ relative to our codec with VBS prediction. Similar observations can be 


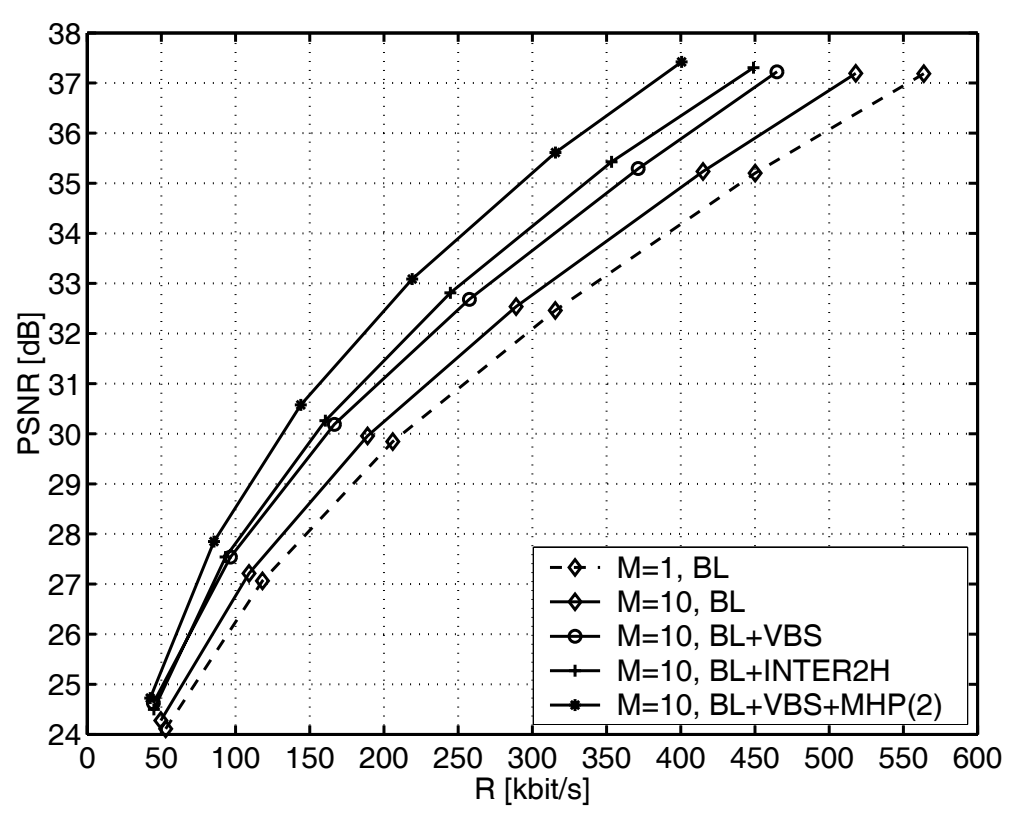

Figure 4.4: Average luminance PSNR over total rate for the sequence Mobile 85 Calendar. Superimposed and variable block size prediction can be successfully combined for compression. $M=10$ reference pictures are utilized for prediction.

made for the sequence Foreman at $120 \mathrm{kbit} / \mathrm{s}$. Superimposed prediction on macroblocks gains about $1 \mathrm{~dB}$ over baseline prediction for $M=10$ (See Fig. 4.3). Performing superimposed prediction additionally on $8 \times 8$ blocks, the gain is about $0.9 \mathrm{~dB}$ compared to the codec with VBS and $M=10$ reference pictures.

Please note that the coding efficiency for the sequences Foreman (Fig. 4.3) and Mobile 8 Calendar (Fig. 4.4) is comparable for VBS prediction (BL+VBS) and superimposed prediction with two hypotheses (BL+INTER2H) over the range of bit-rates considered. Superimposed prediction utilizes just two motion vectors and picture reference parameters compared to four for the INTER4V mode.

For variable block size prediction, four hypotheses provide also no significant improvement over two hypotheses. For example, the superposition codec with VBS and four hypotheses achieves just up to $0.3 \mathrm{~dB}$ gain over the codec with two hypotheses for the sequence Mobile \& Calendar.

In summary, superimposed prediction works efficiently for both $16 \times 16$ and $8 \times 8$ blocks. The savings due to superimposed prediction are observed in the baseline mode as well as in the VBS prediction mode. Hence, the hypothesis selection algorithm in Fig. 2.6 is able to find two prediction signals on $M=10$ reference frames which are combined more efficiently than just one prediction signal from these reference frames.

\subsubsection{Multiple Hypotheses and Multiple Reference Pictures}

The results presented so far are obtained for superimposed motion-compensated prediction with $M=10$ reference pictures in sliding window buffering mode. In this section, the 
influence of multiple reference frames on the superposition codec is investigated [FWG00a]. It is demonstrated that two hypotheses chosen only from the prior decoded frame, i.e. $M=1$ also improve coding efficiency. Additionally, the use of multiple reference frames enhances the efficiency of the superposition codec.

Figs. 4.5 and 4.6 show the bit-rate savings at $35 \mathrm{~dB}$ PSNR of the decoded luminance signal over the number of reference frames $M$ for the sequences Foreman and Mobile \& Calendar, respectively. We compute PSNR vs. bit-rate curves by varying the quantization parameter and interpolate intermediate points by a cubic spline. The performance of the codec with variable block size prediction (VBS) is compared to the superposition codec with two hypotheses (VBS+MHP(2)). Results are depicted for $M=1,2,5,10$, and 20 .

Figs. 4.7 and 4.8 show the same experiment as Figs. 4.5 and 4.6 but depict the absolute bit-rate over the number of reference pictures $M$ for the sequences Foreman and Mobile $\&$ Calendar, respectively. The relative bit-rate savings with two hypotheses are given.

The superposition codec with $M=1$ reference frame has to choose both prediction signals from the previous decoded frame. The superposition codec with VBS saves $7 \%$ for the sequence Foreman and $9 \%$ for the sequence Mobile $\&$ Calendar when compared to the VBS codec with one reference frame. For $M>1$, more than one reference frame is allowed for each prediction signal. The reference frames for both hypotheses are selected by the rate-constrained superimposed motion estimation algorithm. The picture reference parameter allows also the special case that both hypotheses are chosen from the same reference frame. The rate constraint is responsible for the trade-off between prediction quality and bit-rate. For $M=20$ reference frames, the superposition codec with VBS saves $25 \%$ for the sequence Foreman and $31 \%$ for the sequence Mobile $\&$ Calendar when compared to the VBS codec with one reference frame. For the same number of reference frames, the VBS codec saves about $15 \%$ for both sequences. The superposition codec with VBS benefits when being combined with multiframe prediction so that the savings are more than additive. The bit-rate savings saturate for 20 reference frames for both sequences.

In Section 3.4.4 we model multiframe motion compensation by forward-adaptive hypothesis switching. When being combined with complementary hypotheses motion compensation, we observe that the bit-rate savings by complementary hypotheses motion compensation over single hypothesis motion compensation increases with the number of motion-compensated signals $M$. For that, $N=2$ complementary hypotheses are sufficient. This theoretical result is consistent with the previous experimental results.

Figs. 4.9 and 4.10 depict the average luminance PSNR over the total bit-rate for the sequences Foreman and Mobile $\mathscr{G}$ Calendar. The superposition codec with variable block size $(\mathrm{VBS}+\mathrm{MHP}(2))$ is compared to the variable block size codec (VBS) for $M=1$ and $M=20$ reference frames. We can observe in these figures that superimposed prediction in combination with multiframe motion compensation achieves coding gains up to $1.8 \mathrm{~dB}$ for Foreman and $2.8 \mathrm{~dB}$ for Mobile $\mathscr{G}$ Calendar. It is also observed that the use of multiple reference frames enhances the efficiency of superimposed motion-compensated prediction for video compression. 


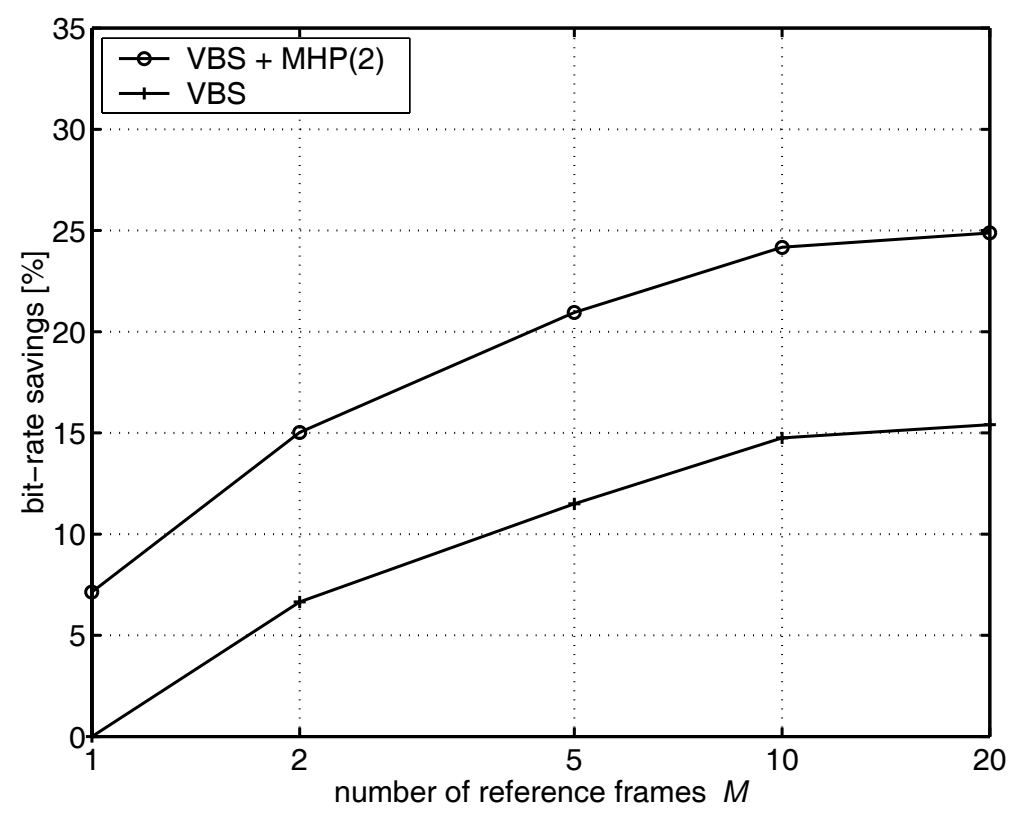

Figure 4.5: Bit-rate savings at $35 \mathrm{~dB}$ PSNR over the number of reference pictures $M$ for the sequence Foreman. For variable block sizes, the performance of the superposition codec is compared to the reference codec.

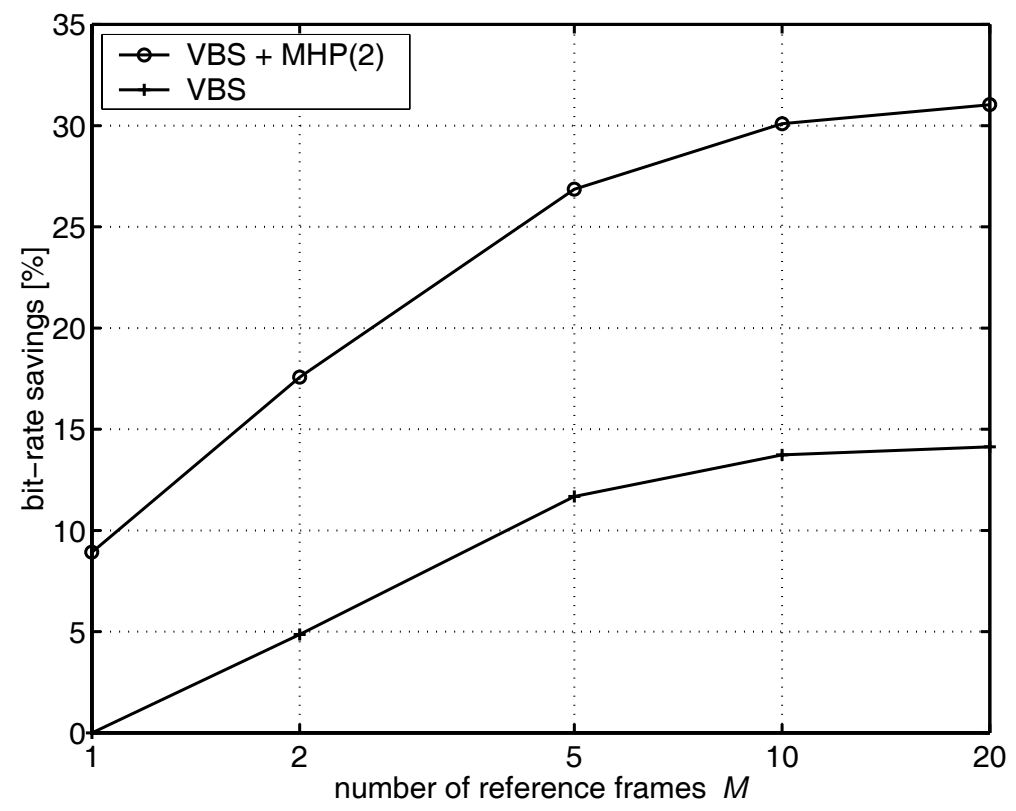

Figure 4.6: Bit-rate savings at $35 \mathrm{~dB}$ PSNR over the number of reference pictures $M$ for the sequence Mobile $\&$ Calendar. For variable block sizes, the performance of the superposition codec is compared to the reference codec. 


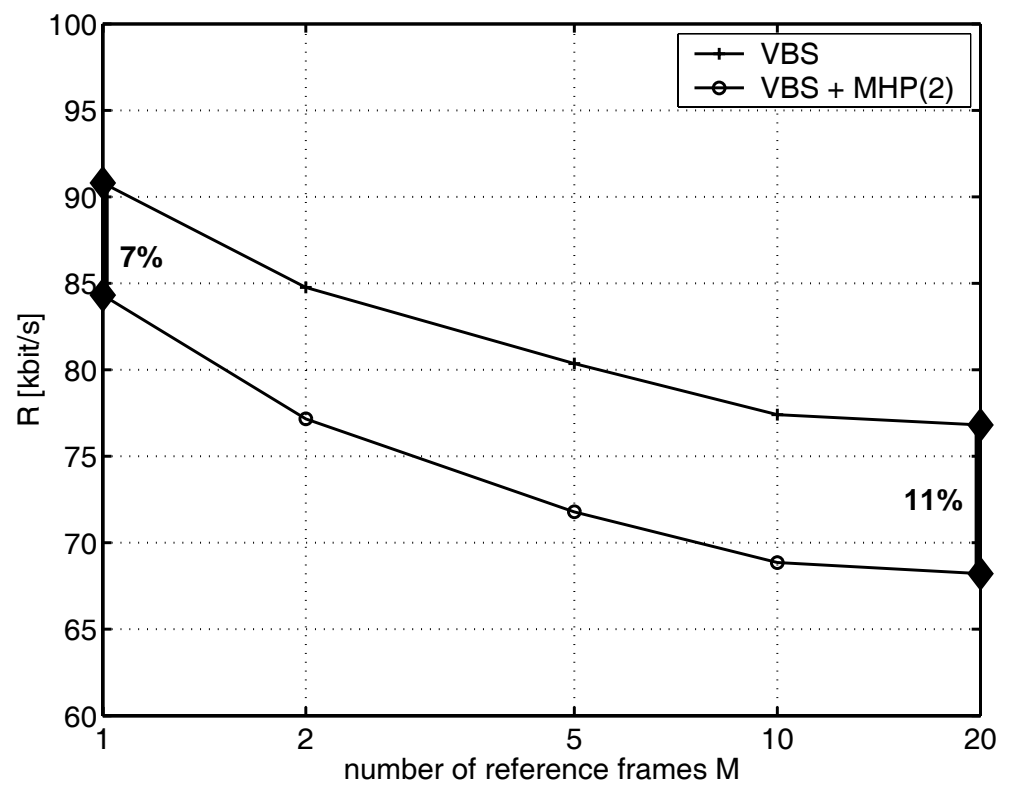

Figure 4.7: Absolute bit-rate at $35 \mathrm{~dB}$ PSNR over the number of reference pictures $M$ for the sequence Foreman. For variable block sizes, the performance of the superposition codec is compared to the reference codec.

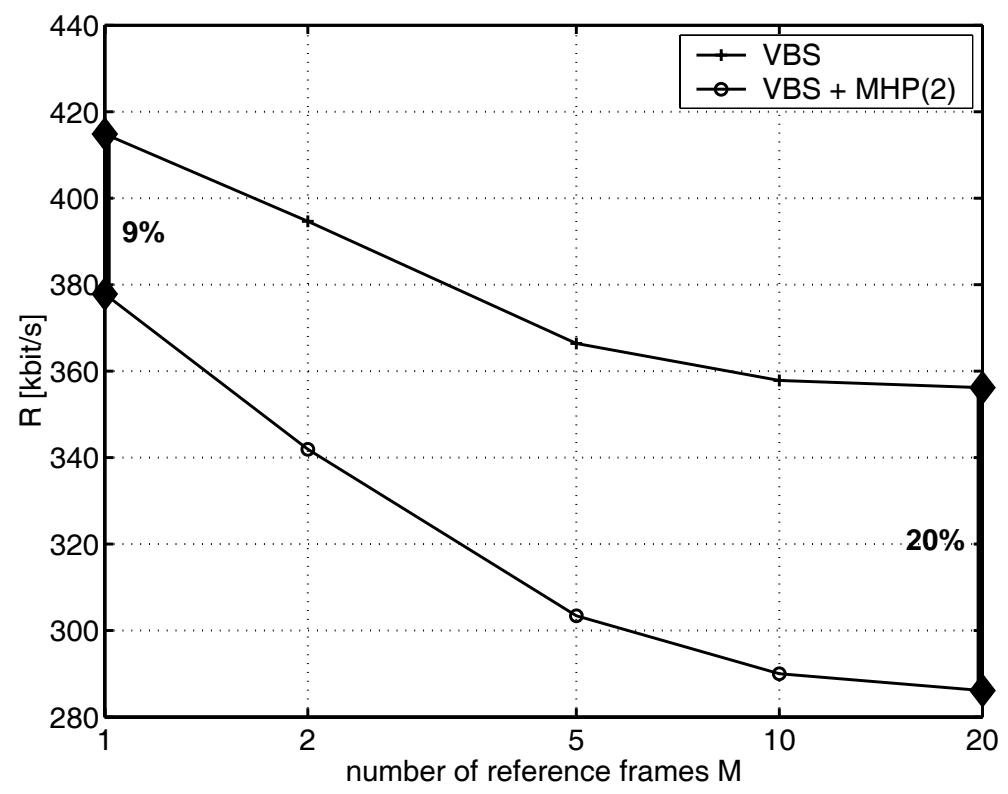

Figure 4.8: Absolute bit-rate at $35 \mathrm{~dB}$ PSNR over the number of reference pictures $M$ for the sequence Mobile \& Calendar. For variable block sizes, the performance of the superposition codec is compared to the reference codec. 


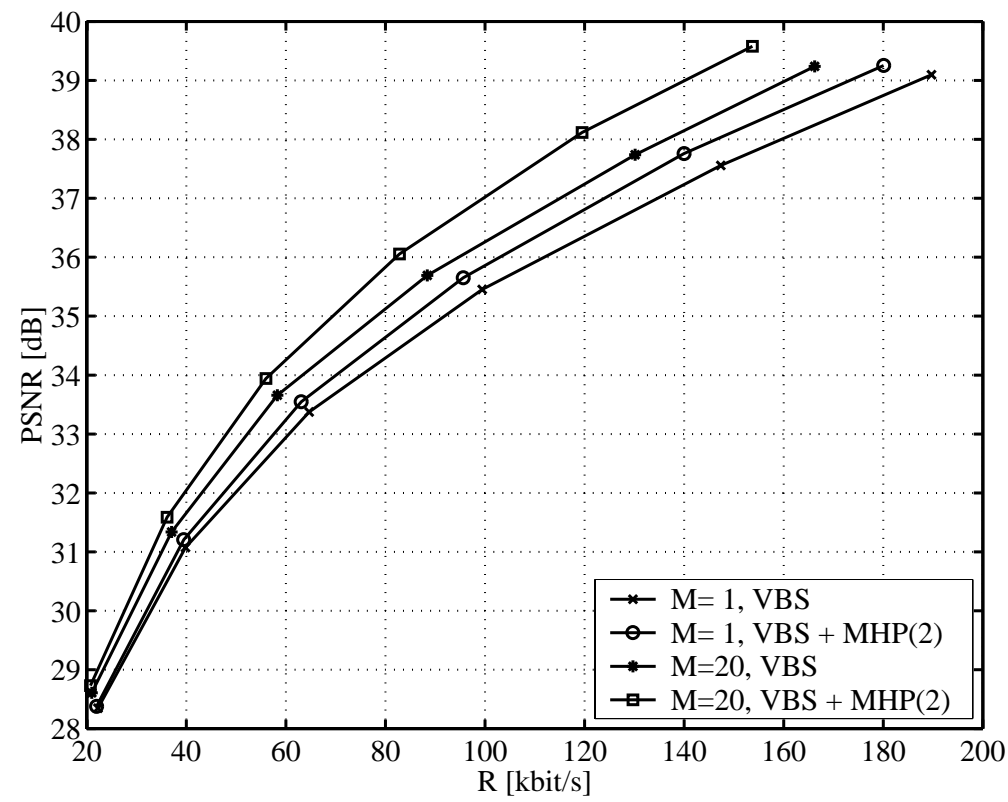

Figure 4.9: Average luminance PSNR over total rate for the sequence Foreman. The performance of the superposition codec with variable block size is depicted for $M=1$ and $M=20$ reference frames.

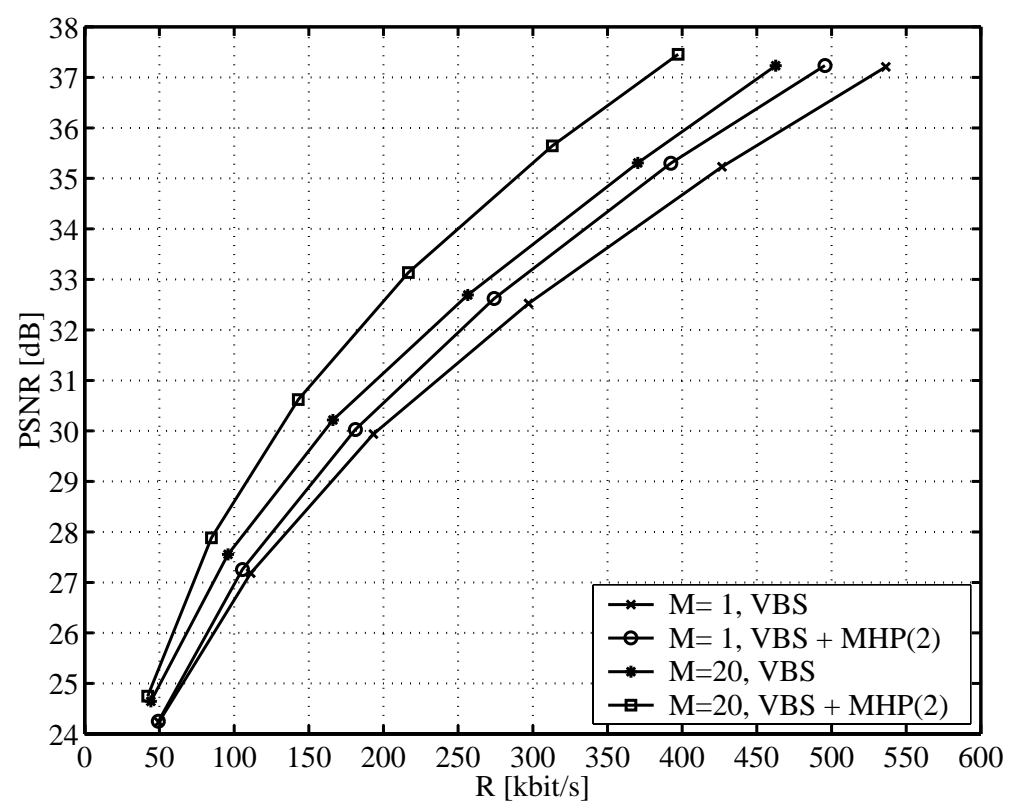

Figure 4.10: Average luminance PSNR over total rate for the sequence Mobile 8 Calendar. The performance of the superposition codec with variable block size is depicted for $M=1$ and $M=20$ reference frames. 


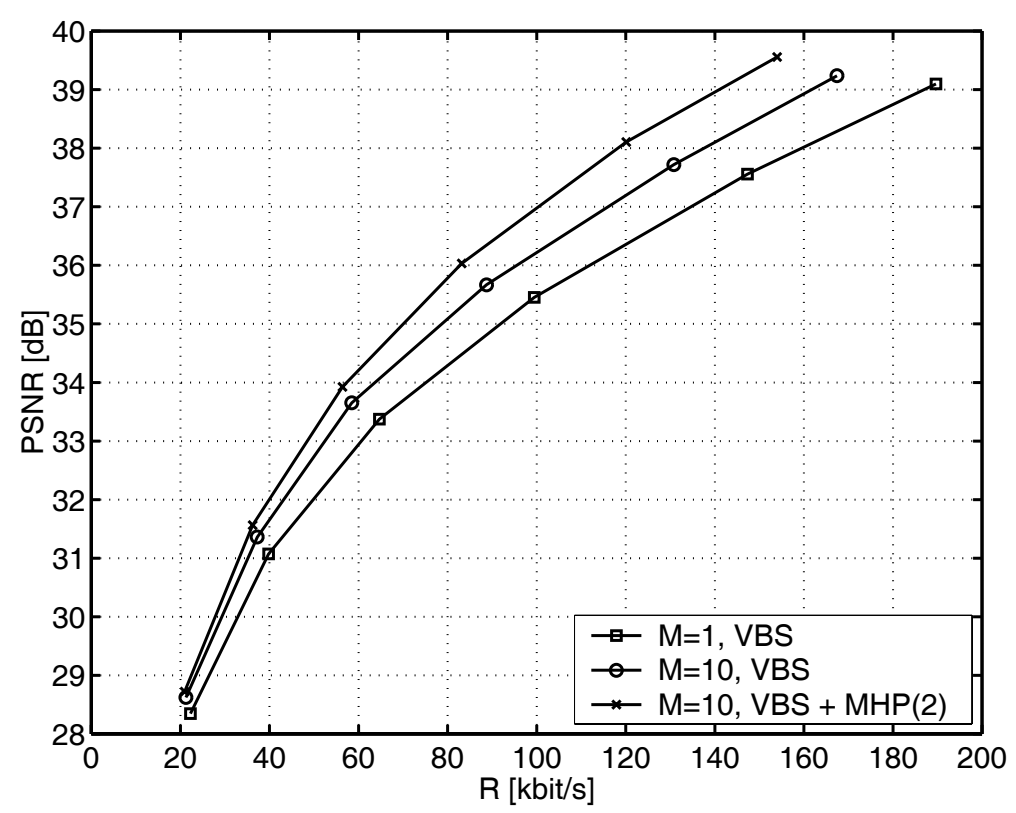

Figure 4.11: Average luminance PSNR over total rate for the sequence Foreman. The performance of the superposition codec with variable block size and multiframe motion compensation is compared to the reference codec.

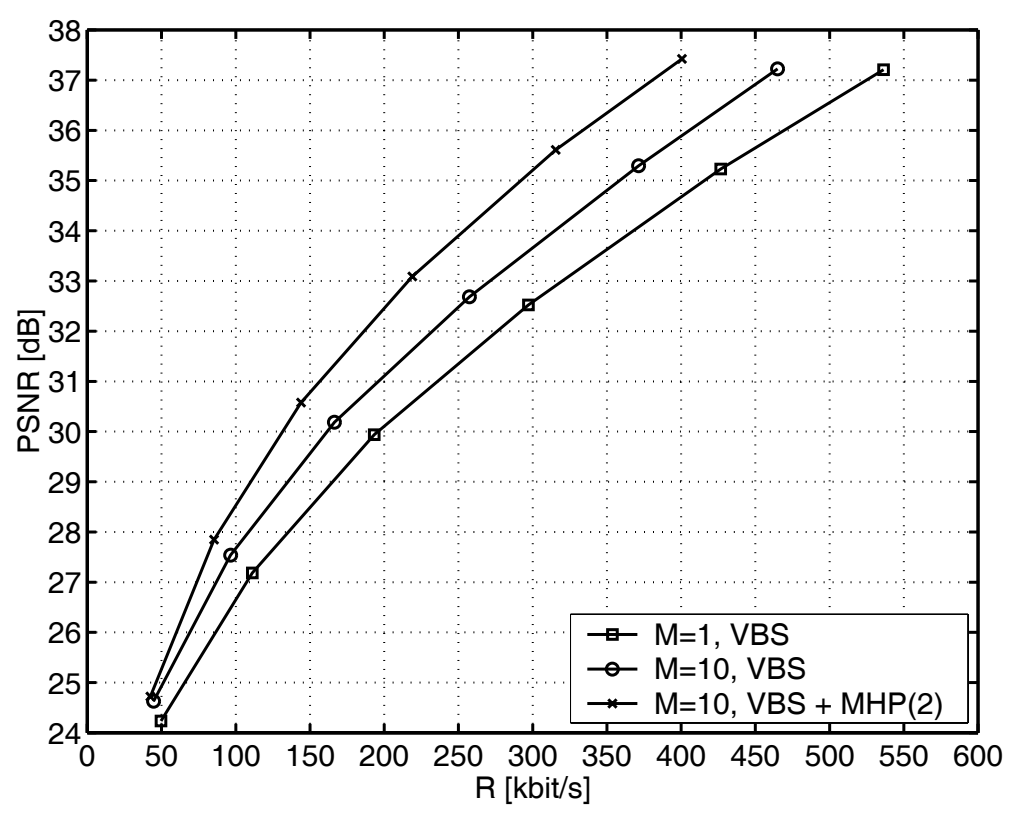

Figure 4.12: Average luminance PSNR over total rate for the sequence Mobile $\&$ Calendar. The performance of the superposition codec with variable block size and multiframe motion compensation is compared to the reference codec.

Finally, Figs. 4.5 and 4.6 suggest that a frame memory of $M=10$ provides a good trade-off between encoder complexity and compression efficiency for our superposition codec. Therefore, Figs. 4.11, 4.12, 4.13, and 4.14 compare the superposition codec with 


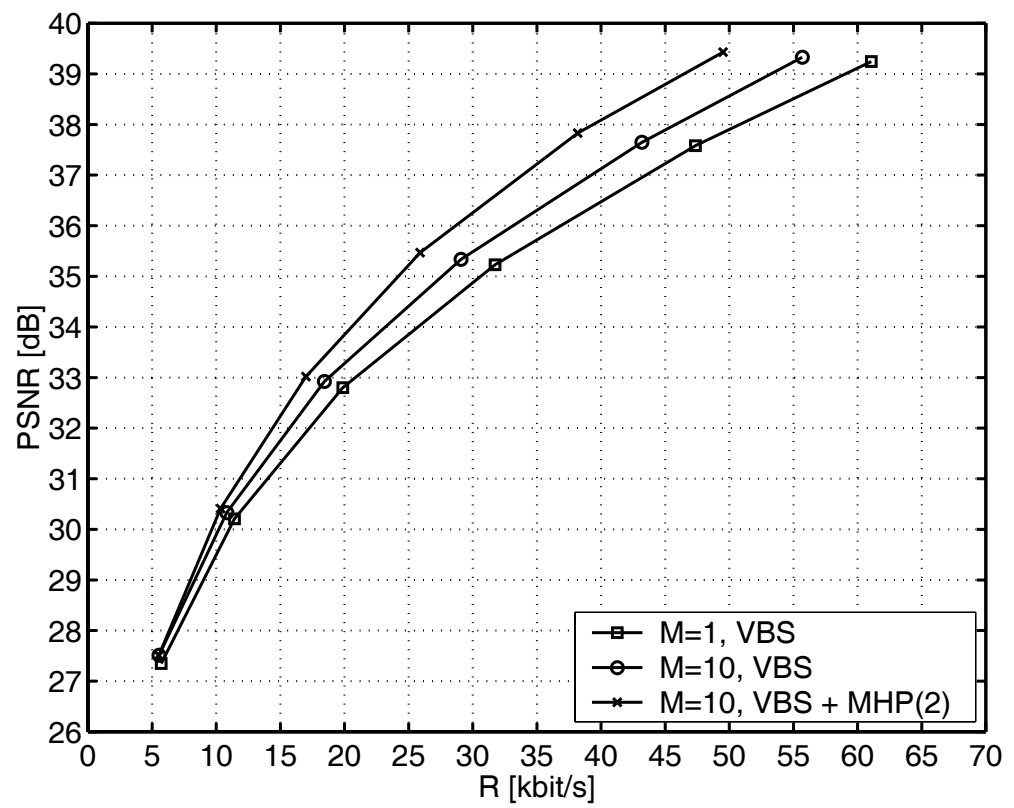

Figure 4.13: Average luminance PSNR over total rate for the sequence Sean. The performance of the superposition codec with variable block size and multiframe motion compensation is compared to the reference codec.

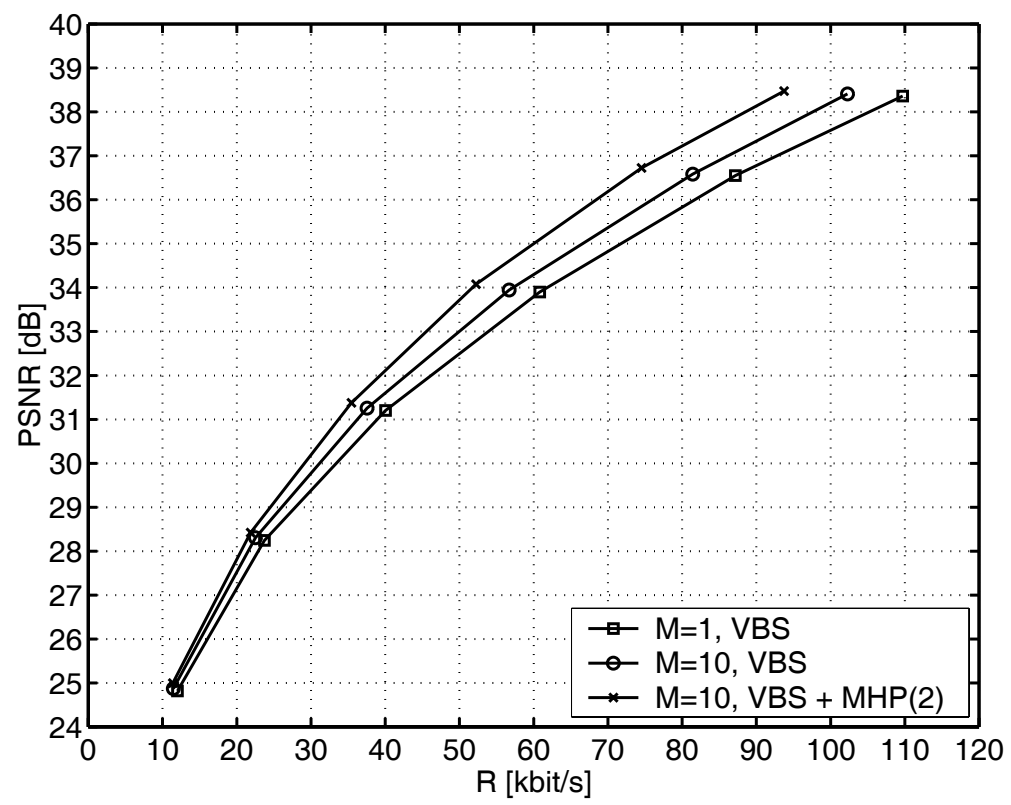

Figure 4.14: Average luminance PSNR over total rate for the sequence Weather. The performance of the superposition codec with variable block size and multiframe motion compensation is compared to the reference codec.

variable block size and frame memory $M=10$ to the reference codec with frame memory $M=1$ and $M=10$ for the sequences Foreman, Mobile \& Calendar, Sean, and Weather, respectively. For each sequence the average luminance PSNR is depicted over the total 
bit-rate. The superposition codec with multiframe motion compensation achieves coding gains up to $1.8 \mathrm{~dB}$ for Foreman, $2.7 \mathrm{~dB}$ for Mobile \& Calendar, $1.6 \mathrm{~dB}$ for Sean, and 1.5 $\mathrm{dB}$ for Weather compared to the reference codec with frame memory $M=1$. The gain by multiframe prediction and superimposed prediction is comparable for the presented sequences.

\subsection{Conclusions}

In our experiments, we observe that variable block size and superimposed prediction can be combined successfully. Superimposed prediction works efficiently for both $16 \times 16$ and $8 \times 8$ blocks. Multiframe motion compensation enhances the efficiency of superimposed prediction. The superposition gain and the multiframe gain do not only add up; superimposed prediction benefits from hypotheses that can be chosen from different reference frames. Superimposed motion-compensated prediction with two hypotheses and ten reference frames achieves coding gains up to $2.7 \mathrm{~dB}$, or equivalently, bit-rate savings up to $30 \%$ for the sequence Mobile $\&$ Calendar when compared to the reference codec with one reference frame. Therefore, superimposed prediction with multiframe and variable block size motion compensation is very efficient and practical for video compression. 


\section{ITU-T Rec. H.264 and Generalized B-Pictures}

\section{$5.1 \quad$ Introduction}

This chapter discusses B-pictures in the context of the draft H.264 video compression standard. B-pictures are pictures in a motion video sequence that are encoded using both past and future pictures as references. The prediction is obtained by a linear combination of forward and backward prediction signals usually obtained with motion compensation. However, such a superposition is not necessarily limited to forward and backward prediction signals as investigated in Chapter 4. For example, a linear combination of two forward prediction signals can also be efficient in terms of compression efficiency. The prediction method which linearly combines motion-compensated signals regardless of the reference picture selection will be referred to as superimposed motion-compensated prediction. The concept of reference picture selection [WZG99], also called multiple reference picture prediction, is utilized to allow prediction from both temporal directions. In this particular case, a bidirectional picture reference parameter addresses both past and future reference pictures [Han00]. This generalization in terms of picture reference selection and linearly combined prediction signals is reflected in the term generalized B-pictures and is part of the emerging H.264 video compression standard [ITU01]. It is desirable that an arbitrary pair of reference pictures can be signaled to the decoder [FG01a, FG01d]. This includes the classical combination of forward and backward prediction signals but also allows forward/forward as well as backward/backward pairs. When combining the two most previous pictures, a functionality similar to the dual-prime mode in MPEG-2 [ISO96, PMGF96] is achieved, where top and bottom fields are averaged to form the final prediction.

B-pictures in H.264 have been improved in several ways compared to B-pictures in MPEG-2 [ISO96] and H.263 [ITU00]. The block size for motion compensation can range from $16 \times 16$ to $4 \times 4$ pixels and the direct mode with weighted blending allows not only a scaling of the motion vectors but also a weighting of the prediction signal. The ongoing H.264 development will also provide improved H.263 Annex U functionality. H.263 Annex U, Enhanced Reference Picture Selection, already allows multiple reference pictures for forward prediction and two-picture backward prediction in B-pictures. When choosing between the most recent and the subsequent reference picture, the multiple reference picture selection capability is very limited. Utilizing multiple prior and subsequent reference pictures improves the compression efficiency of H.263 B-pictures.

The H.264 test model software TML-9 uses only inter pictures as reference pictures 
to predict the B-pictures. Beyond the test model software TML-9, and different from past standards, the multiple reference picture framework in $\mathrm{H} .264$ also allows previously decoded B-pictures to be used as reference to improve prediction efficiency [KKS01]. Bpictures can be utilized to establish an enhancement layer in a layered representation and allow temporal scalability [YLHH00]. That is, decoding of a sequence at more than one frame rate is achievable. In addition to this functionality, B-pictures generally improve the overall compression efficiency as compared to that of inter pictures only [Lil99]. On the other hand, they increase the time delay due to multiple future reference pictures. But this disadvantage is not critical in applications like Internet streaming and multimedia storage for entertainment purposes.

The outline of this chapter is as follows: Section 5.2 introduces B-picture prediction modes. After an overview, direct and superposition mode are discussed in more detail and a rate-distortion performance comparison of three mode classes is provided. Section 5.3 elaborates on superimposed prediction. The difference between bidirectional and superposition mode is outlined and quantified in experimental results. In addition, the efficiency of two combined forward prediction signals is also investigated. Finally, both entropy coding schemes of H.264 are investigated with respect to the superposition mode. Encoder issues are detailed in Section 5.4, which covers rate-constrained mode decision, motion estimation, and superimposed motion estimation. In addition, the improvement of the overall rate-distortion performance with B-pictures is discussed.

\subsection{Prediction Modes for B-Pictures}

\subsubsection{Overview}

The macroblock modes for B-pictures allow intra and inter coding. The intra-mode macroblocks specified for inter pictures are also available for B-pictures. The inter-mode macroblocks are especially tailored to B-picture use. As for inter pictures, they utilize seven block size types as depicted in Fig. 5.1 to generate the motion-compensated macroblock prediction signal. In addition, the usage of the reference picture set available for predicting the current B-picture is suited to its temporally non-causal nature.

In contrast to the previously mentioned inter-mode macroblocks which signal motion vector data according to its block size as side-information, the direct-mode macroblock does not require such side-information but derives reference frame, block size, and motion vector data from the subsequent inter picture. This mode linearly combines two prediction signals. One prediction signal is derived from the subsequent inter picture, the other from a previous picture.

A linear combination of two motion-compensated prediction signals with explicit side-information is accomplished by the superposition-mode. Existing standards with Bpictures utilize the bidirectional mode, which only allows the combination of a previous and subsequent prediction signal. The superposition mode generalizes this concept and supports not only the already mentioned forward/backward prediction pair, but also forward/forward and backward/backward pairs. 


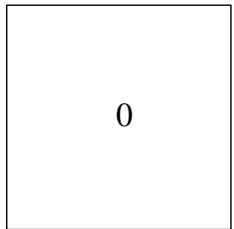

$16 \times 16$

\begin{tabular}{|l|l|l|l|}
\hline 0 & 1 & 2 & 3 \\
\hline 4 & 5 & 6 & 7 \\
\hline
\end{tabular}

$8 \times 4$

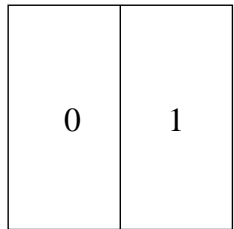

$16 \times 8$

\begin{tabular}{|l|l|}
\hline 0 & 1 \\
\hline 2 & 3 \\
\hline 4 & 5 \\
\hline 6 & 7 \\
\hline
\end{tabular}

$4 \times 8$

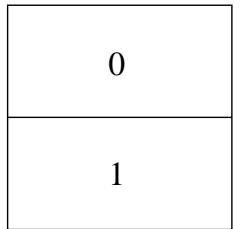

$8 \times 16$

\begin{tabular}{|l|l|}
\hline 0 & 1 \\
\hline 2 & 3 \\
\hline
\end{tabular}

$8 \times 8$

Figure 5.1: Block size types for the motion-compensated macroblock prediction signal.

\subsubsection{Direct Mode}

The direct mode uses bidirectional prediction and allows residual coding of the prediction error. The forward and backward motion vectors of this mode are derived from the motion vectors used in the corresponding macroblocks of the subsequent reference picture. The same number of motion vectors are used. To calculate prediction blocks, the forward and backward motion vectors are used to obtain appropriate blocks from reference pictures and then these blocks are linearly combined. Using multiple reference picture prediction, the forward reference picture for the direct mode is the same as the one used for the corresponding macroblock in the subsequent inter picture. The forward and backward motion vectors for direct mode macroblocks are calculated as

$$
\begin{aligned}
M V_{F} & =\frac{T R_{B}}{T R_{D}} M V \\
M V_{B} & =\frac{T R_{B}-T R_{D}}{T R_{D}} M V
\end{aligned}
$$

where $M V_{F}$ is the forward motion vector, $M V_{B}$ is the backward motion vector, and $M V$ represents the motion vectors in the corresponding macroblock in the subsequent inter picture. $T R_{D}$ is the temporal distance between the previous and the next inter picture, and $T R_{B}$ is the distance between the current picture and the previous inter picture. It should be noted that when multiple reference picture prediction is used, the reference picture for the motion vector predictions is treated as though it were the most recent previous decoded picture. Thus, instead of using the temporal reference of the exact reference picture to compute the temporal distances $T R_{D}$ and $T R_{B}$, the temporal reference of the most recent previous reference picture is used to compute the temporal distances.

The direct mode in H.264 is improved by weighted blending of the prediction signal [Lil00]. Video content like music videos and movie trailers make frequent use of fading 
transitions from scene to scene. It is very popular in movie trailers to fade each scene to black, and then from black to the next scene. Without weighted blending of the prediction signal, both normal fades and "fades to-black" are hard to encode well without visible compression artifacts. For example, when encoding with a PBBB pattern, the B-pictures in position 1 and 3 suffer from quality degradation relative to the B-pictures in position 2 and the surrounding inter and intra pictures. The weighted blending technique considers how the direct mode motion vectors are derived from scaling the motion vector for the subsequent inter picture, based on the distance between the B-picture and the surrounding pictures, and also weighs the calculation of the prediction block based on this distance, instead of the averaging with equal weights that has been used in all existing standards with B-pictures. The weighted blending technique calculates the prediction block $c$ for direct mode coded macroblocks according to

$$
c=\frac{c_{p}\left(T R_{D}-T R_{B}\right)+c_{s} T R_{B}}{T R_{D}},
$$

where $c_{p}$ is the prediction block from a previous reference picture, and $c_{s}$ is the prediction block from the subsequent reference picture. Sequences without any fades will not suffer from loss of compression efficiency relative to the conventional way to calculate the prediction for direct mode.

\subsubsection{Superposition Mode}

The superposition mode superimposes two macroblock prediction signals with their individual sets of motion vectors. We refer to each prediction signal as a hypothesis. To calculate prediction blocks, the motion vectors of the two hypotheses are used to obtain appropriate blocks from reference pictures and then these blocks are averaged. Each hypothesis is specified by one of the seven block size types as depicted in Fig. 5.1. In addition, each hypothesis is also assigned one picture reference parameter. The motion vectors for each hypothesis are assigned on a block level and all of them refer to that specified reference picture. It is very likely that the hypotheses are chosen from different reference pictures but they can also originate from the same picture. Increasing the number of available reference pictures improves the performance of superimposed motioncompensated prediction as shown in Section 4.3.3 and theoretically discussed in Section 3.4.4. For B-pictures, more details are given in Section 5.3.

\subsubsection{Rate-Distortion Performance of Individual Modes}

The macroblock modes for B-pictures can be classified into four groups:

1. No extra side-information is transmitted for this particular macroblock. This corresponds to the direct mode.

2. Side-information for one macroblock prediction signal is transmitted. The inter modes with block structures according to Fig. 5.1 and bidirectional picture reference parameters belong to this group. 
3. Side-information for two macroblock prediction signals is transmitted to allow superimposed prediction.

4. The last group includes all intra modes, i.e., no inter-frame prediction is used.

In the following, the first three groups which utilize inter-frame prediction are investigated with respect to their rate-distortion performance. The fourth group with the intra modes is negligible. They are available in each experiment but their frequency is very small.

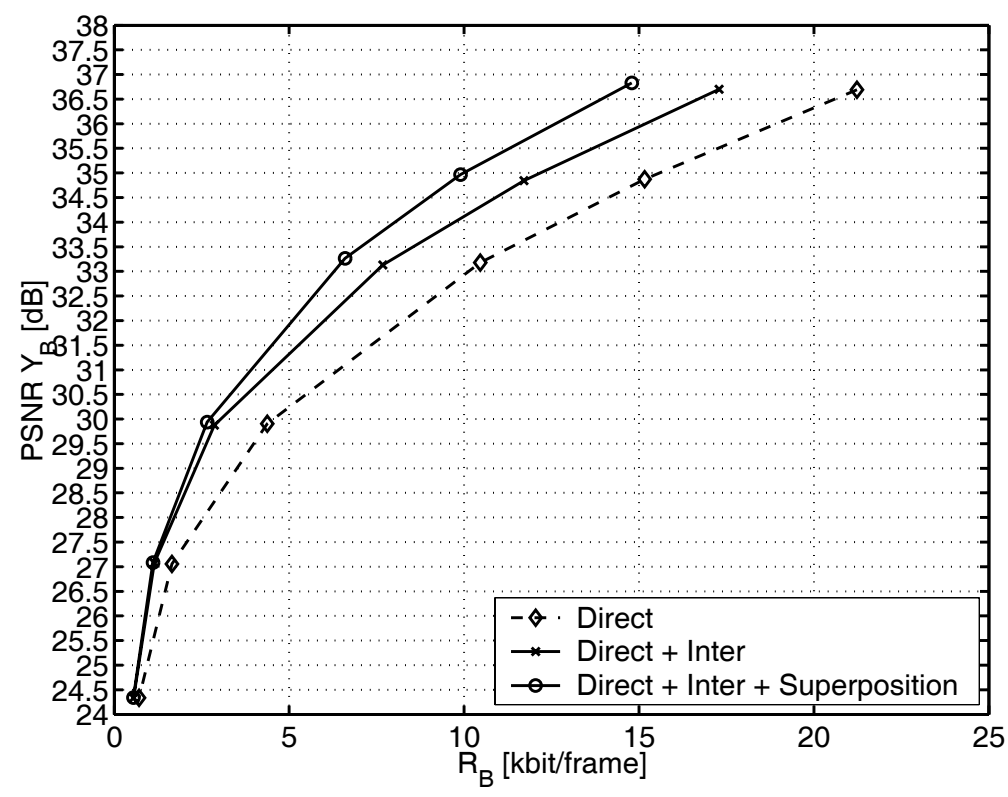

Figure 5.2: PSNR of the B-picture luminance signal vs. B-picture bit-rate for the QCIF sequence Mobile $\&$ Calendar with $30 \mathrm{fps}$. Two B-pictures are inserted after each inter picture. 5 past and 3 subsequent reference pictures are used. The compression efficiency of the B-picture coding modes direct, inter, and superposition are compared.

The rate-distortion performance of the groups direct, inter, and superposition are depicted in Fig. 5.2. The PSNR of the B-picture luminance signal is plotted over the Bpicture bit-rate for the QCIF sequence Mobile $\&$ Calendar. With the direct mode for the B-pictures, the rate-distortion performance at high bit-rates is dominated by the efficiency of the residual encoding. The inter modes improve the compression efficiency approximately by $1 \mathrm{~dB}$ in PSNR at moderate and high bit-rates. At very low bit-rates, the rate-penalty in effect disables the modes in the inter group due to extra side-information. Similar behavior can be observed for the superposition mode. Transmitting two prediction signals increases the side-information. Consequently, the superposition mode improves compression efficiency approximately by $1 \mathrm{~dB}$ in PSNR at high bit-rates.

Corresponding to the rate-distortion performance of the three groups, Fig. 5.3 depicts the relative occurrence of the macroblock modes in B-pictures vs. quantization parameter $Q P_{P}$ for the QCIF sequence Mobile \& Calendar. At $Q P_{P}=28$ (low bit-rate), the direct mode is dominant with approximately $90 \%$ relative occurrence, whereas the superposition 


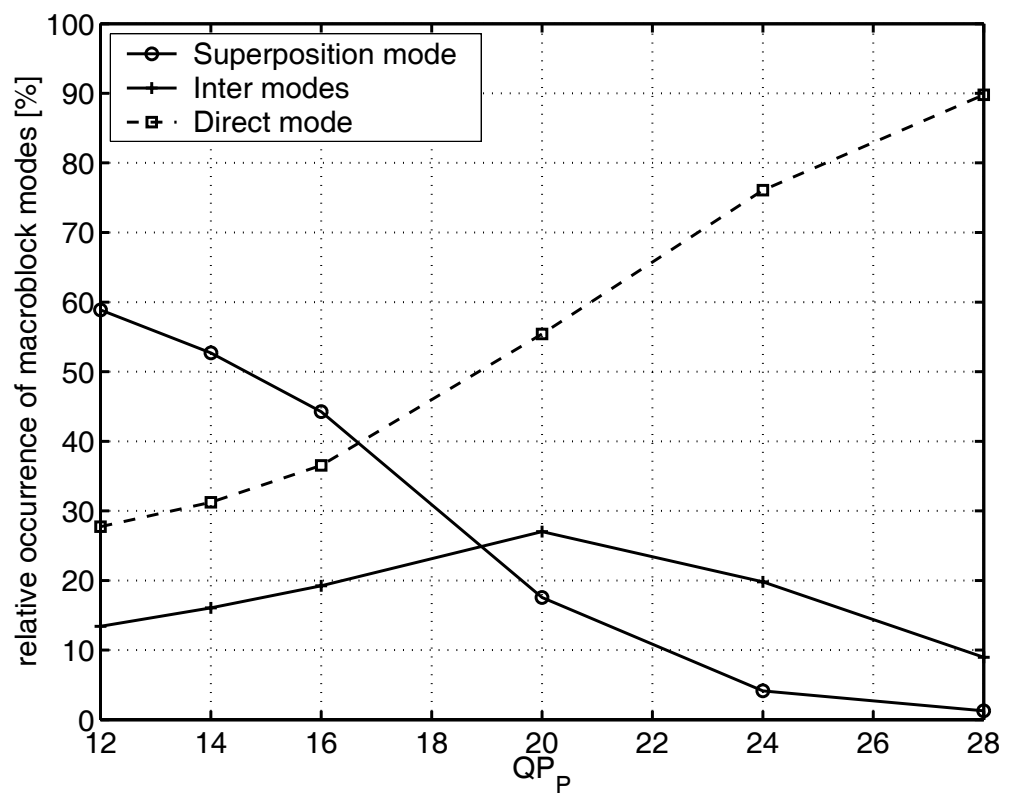

Figure 5.3: Relative occurrence of the macroblock modes in B-pictures vs. quantization parameter for the QCIF sequence Mobile \& Calendar with $30 \mathrm{fps}$. Two B-pictures are inserted after each inter picture. 5 past and 3 subsequent reference pictures are used. The relative frequency of the B-picture macroblock modes direct, inter, and superposition are compared.

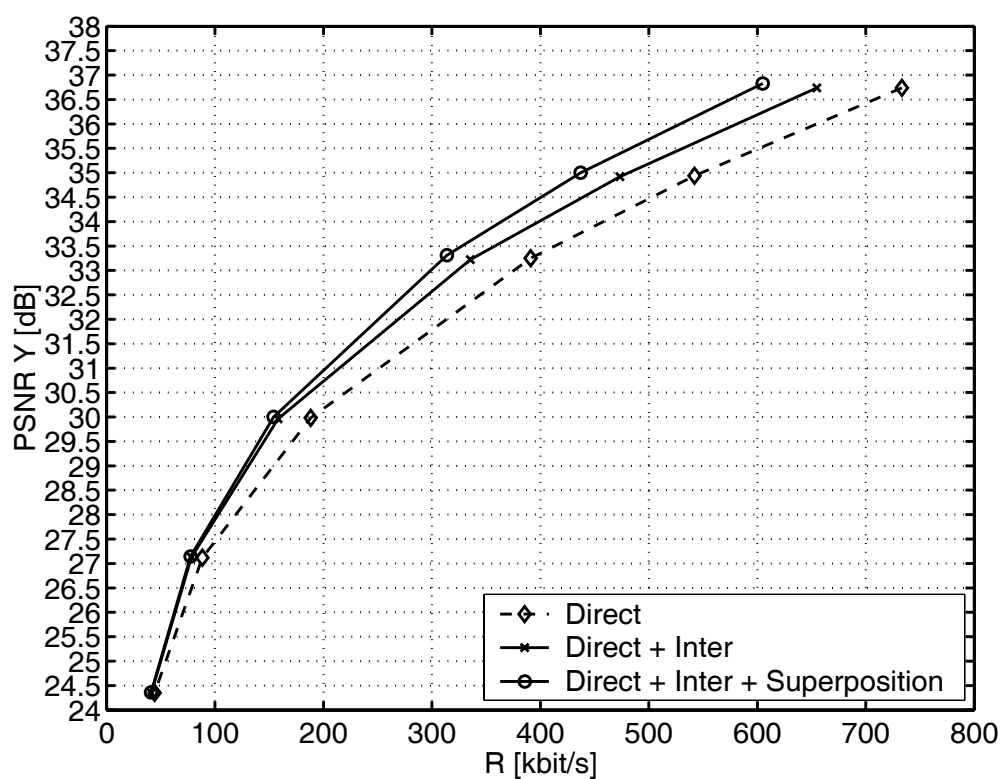

Figure 5.4: PSNR of the luminance signal vs. overall bit-rate for the QCIF sequence Mobile E Calendar with $30 \mathrm{fps}$. Two B-pictures are inserted after each inter picture. 5 past and 3 subsequent reference pictures are used. The compression efficiency of the B-picture coding modes direct, inter, and superposition are compared.

and inter modes are seldom selected due to the rate-constraint. At $Q P_{P}=12$ (high bitrate), the relative occurrence of the direct mode decreases to $30 \%$, whereas the relative 
frequency of the superposition mode increases to $60 \%$. About $10 \%$ of the macroblocks utilize an inter mode.

The influence of the B-picture coding modes direct, inter, and superposition on the overall compression efficiency is depicted in Fig. 5.4 for the QCIF sequence Mobile \& Calendar. The base layer (the sequence of inter pictures) is identical in all three cases and only the B-picture coding modes are selected from the specified classes. For this sequence, the inter modes in the B-pictures improve the overall efficiency approximately by $0.5 \mathrm{~dB}$. The superposition mode adds an additional $0.5 \mathrm{~dB}$ for higher bit-rates.

\subsection{Superimposed Prediction}

\subsubsection{Bidirectional vs. Superposition Mode}

In the following, we will outline the difference between the bidirectional macroblock mode, which is specified in the H.264 test model TML-9 [ITU01], and the superposition mode proposed in [FG01d] and discussed in the previous section. A bidirectional prediction type only allows a linear combination of a forward/backward prediction pair; see Fig. 5.5. The draft TML-9 utilizes multiple reference pictures for forward prediction but allows only backward prediction from the most subsequent reference picture. For bidirectional prediction, independently estimated forward and backward prediction signals are practical but the efficiency can be improved by joint estimation. For superimposed prediction in general, a joint estimation of two hypotheses is necessary as discussed in Chapter 3. An independent estimate might even deteriorate the performance. The test model software TML-9 does not allow a joint estimation of forward and backward prediction signals.

$\mathrm{P}$

$\mathrm{B}$

$\mathrm{P}$

$\mathrm{B}$

$\mathrm{P}$

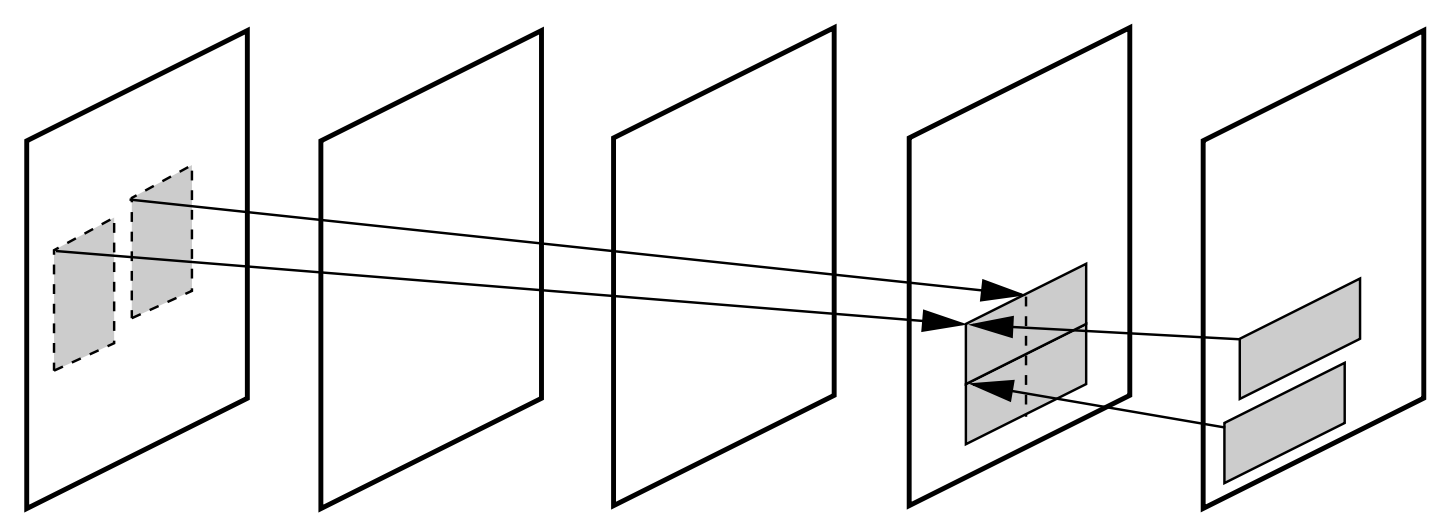

Figure 5.5: A bidirectional prediction mode allows a linear combination of one past and one subsequent macroblock prediction signal. The inter pictures are denoted by $\mathrm{P}$.

The superposition mode removes the restriction of the bidirectional mode to allow only linear combinations of forward and backward pairs [FG02a, GF02]. The additional combinations (forward, forward) and (backward, backward) are obtained by extending an 
unidirectional picture reference syntax element to a bidirectional picture reference syntax element; see Fig. 5.6. With this bidirectional picture reference element, a generic prediction signal, which we call hypothesis, can be formed with the syntax fields for reference frame, block size, and motion vector data.

$\mathrm{P}$

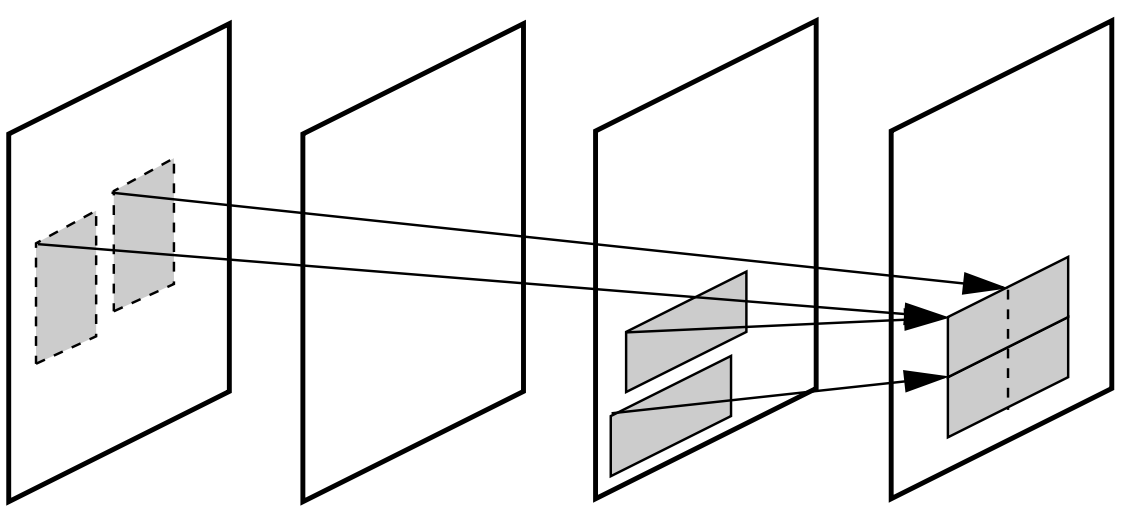

$\mathrm{P}$

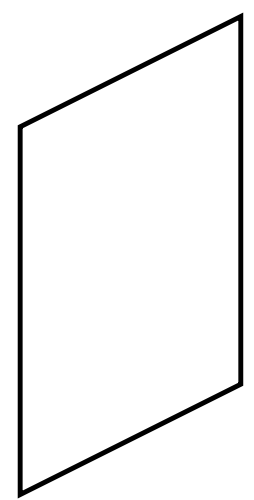

Figure 5.6: The superposition mode allows a linear combination of two past macroblock prediction signals (as depicted), two future macroblock signals, or one past and one future macroblock signal. The inter pictures are denoted by $\mathrm{P}$.

The superposition mode includes the bidirectional prediction mode when the first hypothesis originates from a past reference picture and the second from a future reference picture. The bidirectional mode limits the set of possible reference picture pairs. Not surprisingly a larger set of reference picture pairs improves the coding efficiency of Bpictures.

The following results are based on the H.264 test model TML-9 [ITU01]. For our experiments, the CIF sequences Mobile \& Calendar and Flowergarden are coded at $30 \mathrm{fps}$. We investigate the rate-distortion performance of the superposition mode in comparison with the bidirectional mode when two B-pictures are inserted.

Figs. 5.7 and 5.8 depict the average luminance PSNR from reconstructed B-pictures over the overall bit-rate produced by B-pictures with bidirectional prediction mode and the superposition mode for the sequences Mobile $\&$ Calendar and Flowergarden. The number of reference pictures is chosen to be 1 and 3 future reference pictures with a constant number of 5 past pictures. It can be observed that increasing the total number of reference pictures from $5+1$ to $5+3$ slightly improves compression efficiency. Moreover, the superposition mode outperforms the bidirectional mode and its compression efficiency improves for increasing bit-rate. In the case of the bidirectional mode, jointly estimated forward and backward prediction signals outperform independently estimated signal pairs.

\subsubsection{Two Combined Forward Prediction Signals}

Generalized B-pictures combine both the superposition of prediction signals and the reference picture selection from past and future pictures. In the following, we investigate 


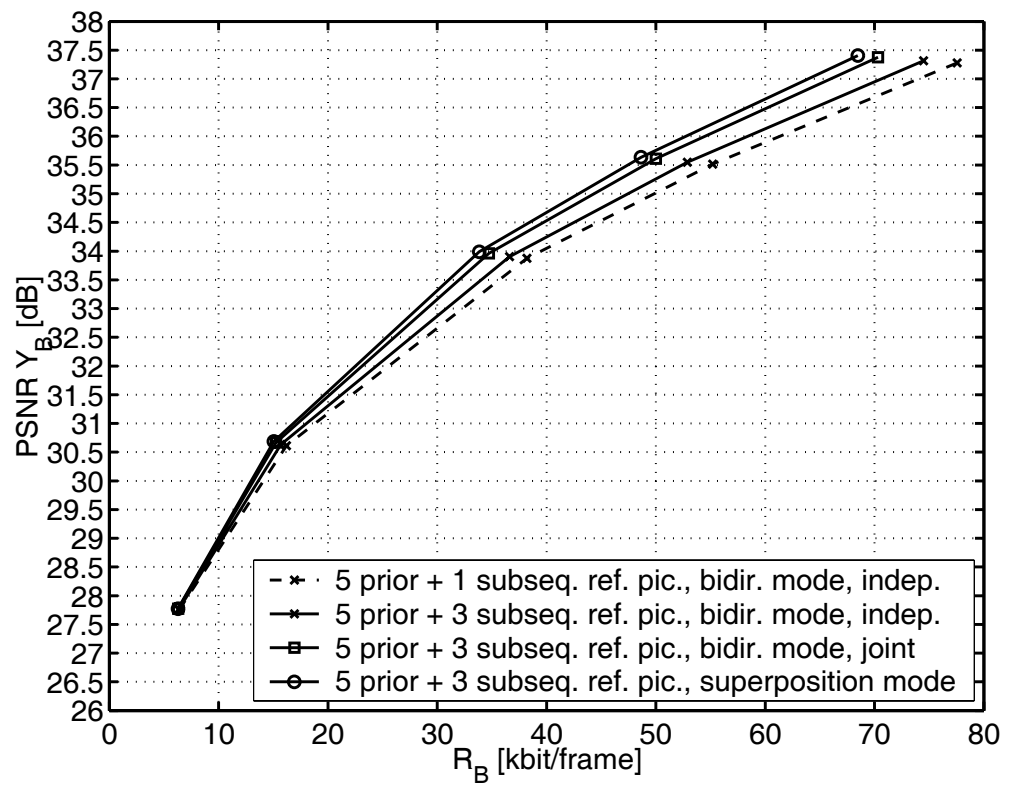

Figure 5.7: PSNR of the B-picture luminance signal vs. B-picture bit-rate for the CIF sequence Mobile $\&$ Calendar with 30 fps. Two B-pictures are inserted after each inter picture. $Q P_{B}=Q P_{P}$. The superposition mode is compared to the bidirectional mode.

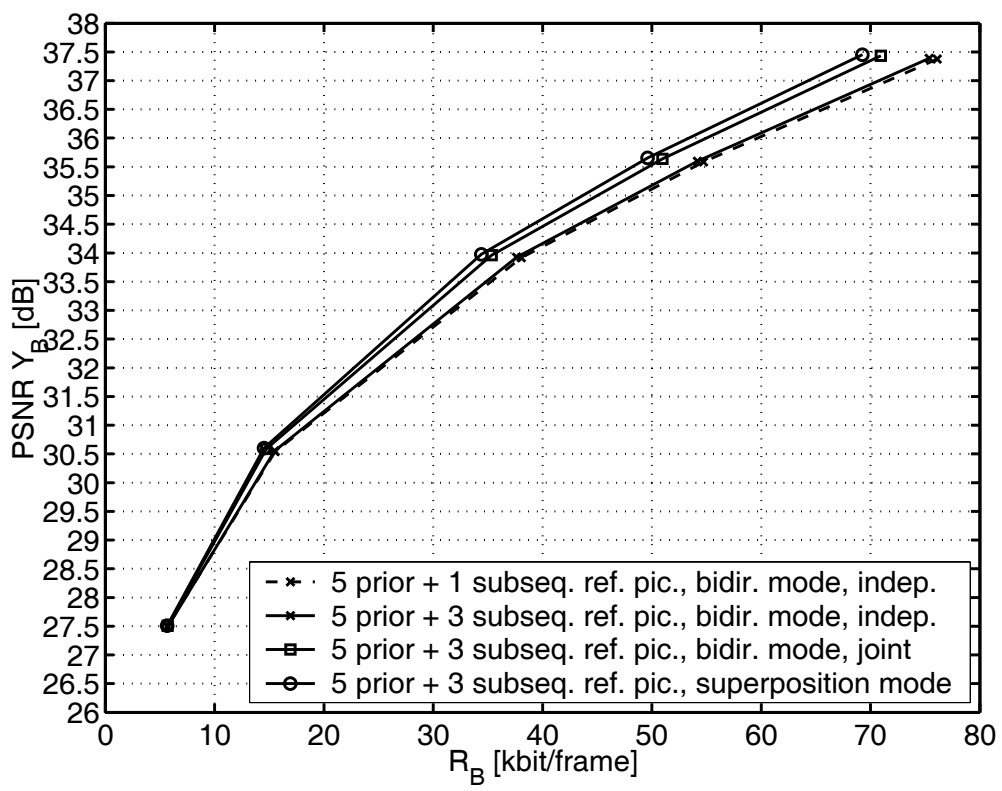

Figure 5.8: PSNR of the B-picture luminance signal vs. B-picture bit-rate for the CIF sequence Flowergarden with $30 \mathrm{fps}$. Two B-pictures are inserted after each inter picture. $Q P_{B}=Q P_{P}$. The superposition mode is compared to the bidirectional mode.

generalized B-pictures with forward-only prediction and utilize them like inter pictures in Chapter 4 for comparison purposes [FG01a, FWG01]. That is, only a unidirectional reference picture parameter which addresses past pictures is permitted. As there is no fu- 
ture reference picture, the direct mode is replaced by the skip mode as specified for inter pictures. The generalized B-pictures with forward-only prediction cause no extra coding delay as they utilize only past pictures for prediction and are also used for reference to predict future pictures.

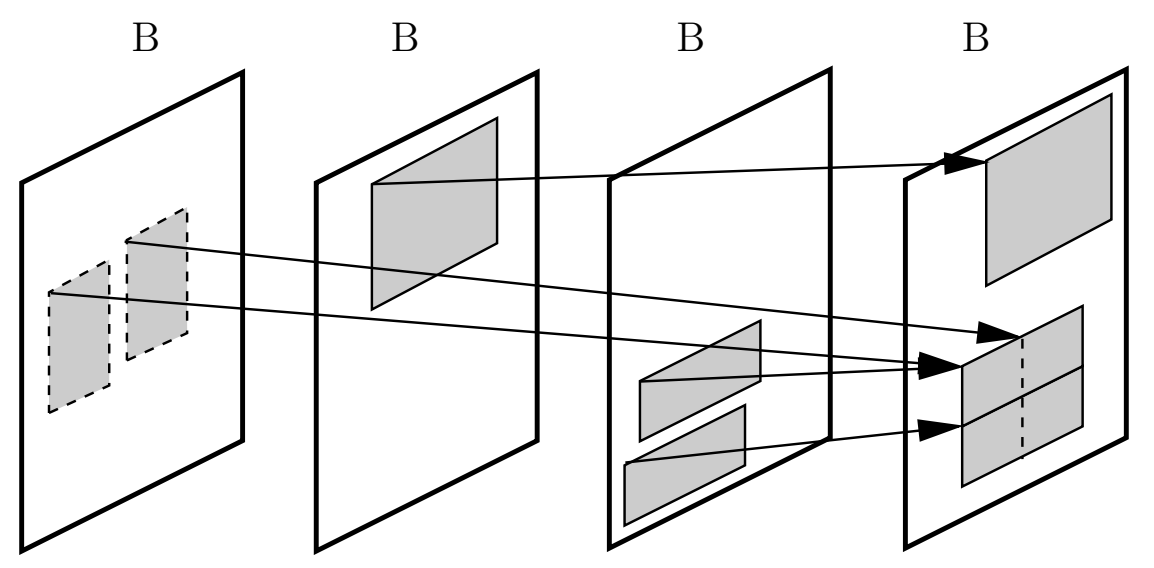

Figure 5.9: Generalized B-pictures with forward-only prediction utilize multiple reference picture prediction and superimposed motion-compensated prediction. The superposition mode uses two hypotheses chosen from past reference pictures.

Fig. 5.9 shows generalized B-pictures with forward-only prediction. They allow multiple reference picture prediction and linearly combined motion-compensated prediction signals with individual block size types. Both hypotheses are just averaged to form the current macroblock. As depicted in Fig. 5.1, the H.264 test model [ITU01] allows seven different block sizes which will be the seven hypotheses types in the superposition mode. The draft H.264 standard allows for inter modes only one picture reference parameter per macroblock and assumes that all sub-blocks can be found on that specified reference picture. This is different from the H.263 standard, where multiple reference picture prediction utilizes picture reference parameters for both macroblocks and $8 \times 8$ blocks [WZG99].

We investigate the rate-distortion performance of generalized B-pictures with forwardonly prediction and compare them to H.264 inter pictures for various numbers of reference pictures. Figs. 5.10 and 5.11 show the bit-rate values at $35 \mathrm{~dB}$ PSNR of the luminance signal over the number of reference pictures $M$ for the CIF sequences Mobile $\&$ Calendar and Tempete, respectively, coded at $30 \mathrm{fps}$. We compute PSNR vs. bit-rate curves by varying the quantization parameter and interpolate intermediate points by a cubic spline. The performance of H.264 inter pictures (IPPP...) and the generalized B-pictures with forward-only prediction (IBBB...) is shown.

The generalized B-pictures with forward-only prediction and $M=1$ reference picture has to choose both hypotheses from the previous picture. For $M>1$, we allow more than one reference picture for each hypothesis. The reference pictures for both hypotheses are selected by the rate-constrained superimposed motion estimation algorithm described in Section 5.4.3. The picture reference parameter allows also the special case that both hypotheses are chosen from the same reference picture. The rate constraint is responsible 


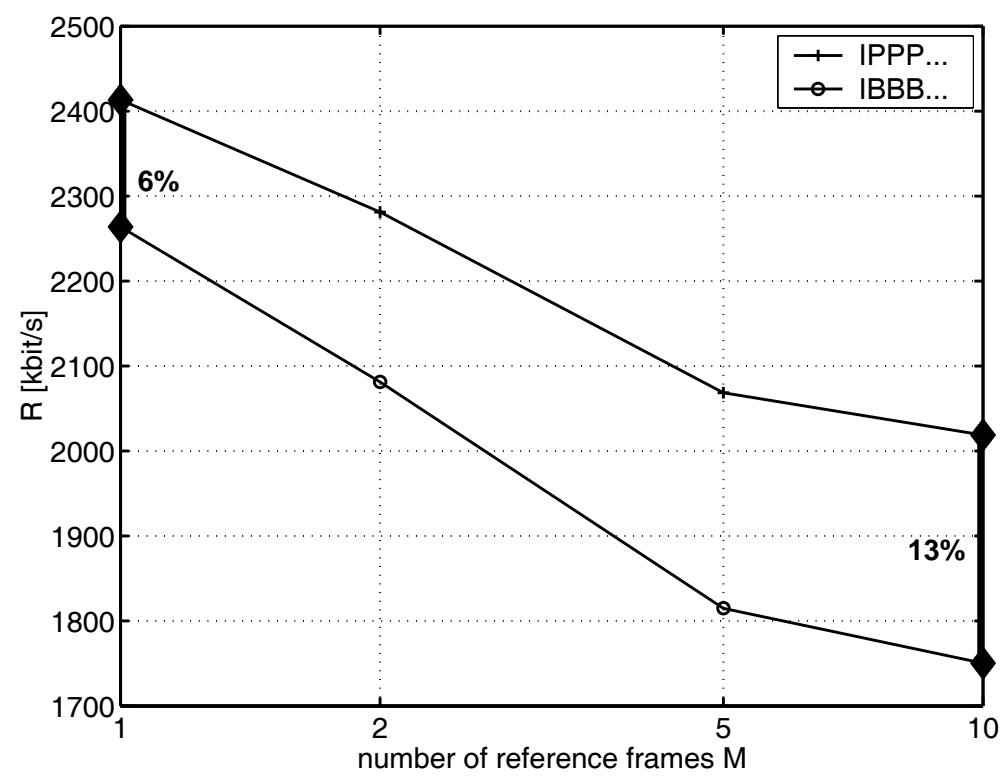

Figure 5.10: Average bit-rate at $35 \mathrm{~dB}$ PSNR vs. number of reference pictures for the CIF sequence Mobile \& Calendar with 30 fps. Generalized B-pictures with forward-only prediction are compared to inter pictures.

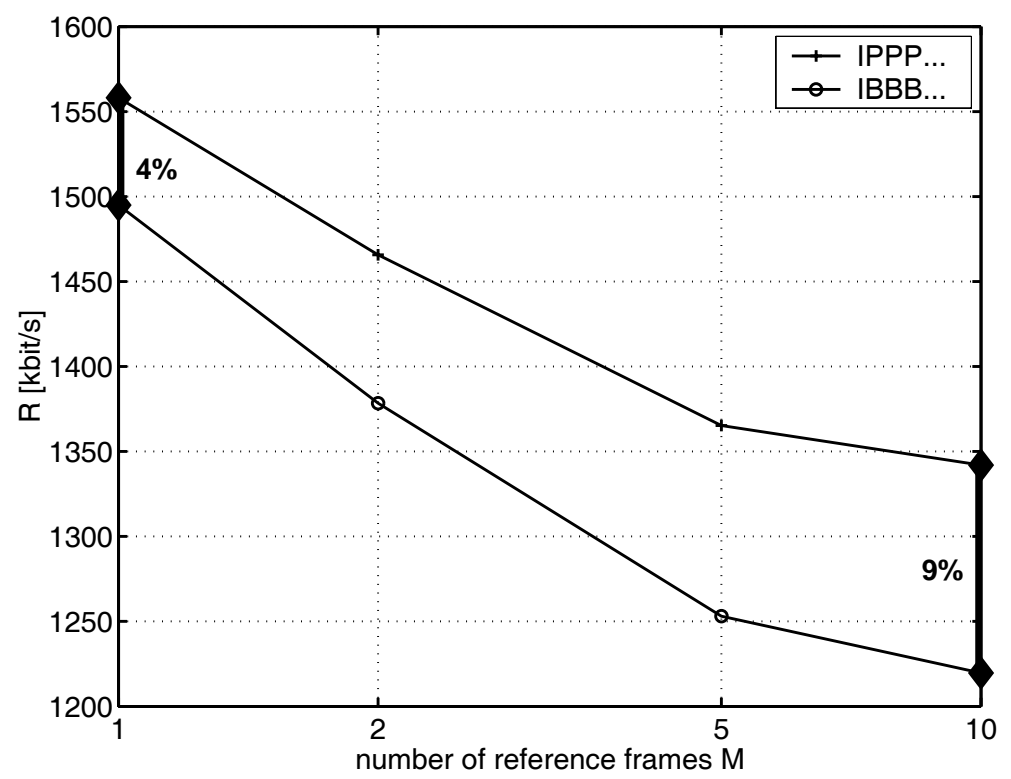

Figure 5.11: Average bit-rate at $35 \mathrm{~dB}$ PSNR vs. number of reference pictures for the CIF sequence Tempete with 30 fps. Generalized B-pictures with forward-only prediction are compared to inter pictures.

for the trade-off between prediction quality and bit-rate. Using the generalized B-pictures with forward-only prediction and $M=10$ reference pictures reduces the bit-rate from 2019 to $1750 \mathrm{kbit} / \mathrm{s}$ when coding the sequence Mobile $\&$ Calendar. This corresponds to $13 \%$ bit-rate savings. The gain by the generalized B-pictures with forward-only prediction and 
just one reference picture is limited to $6 \%$. The gain by the generalized B-pictures over the inter pictures improves for a increasing number of reference pictures as already discussed in Section 4.3.3 for H.263. This observation is independent of the implemented superimposed prediction scheme and is supported by the theoretical investigation in Section 3.4.4.

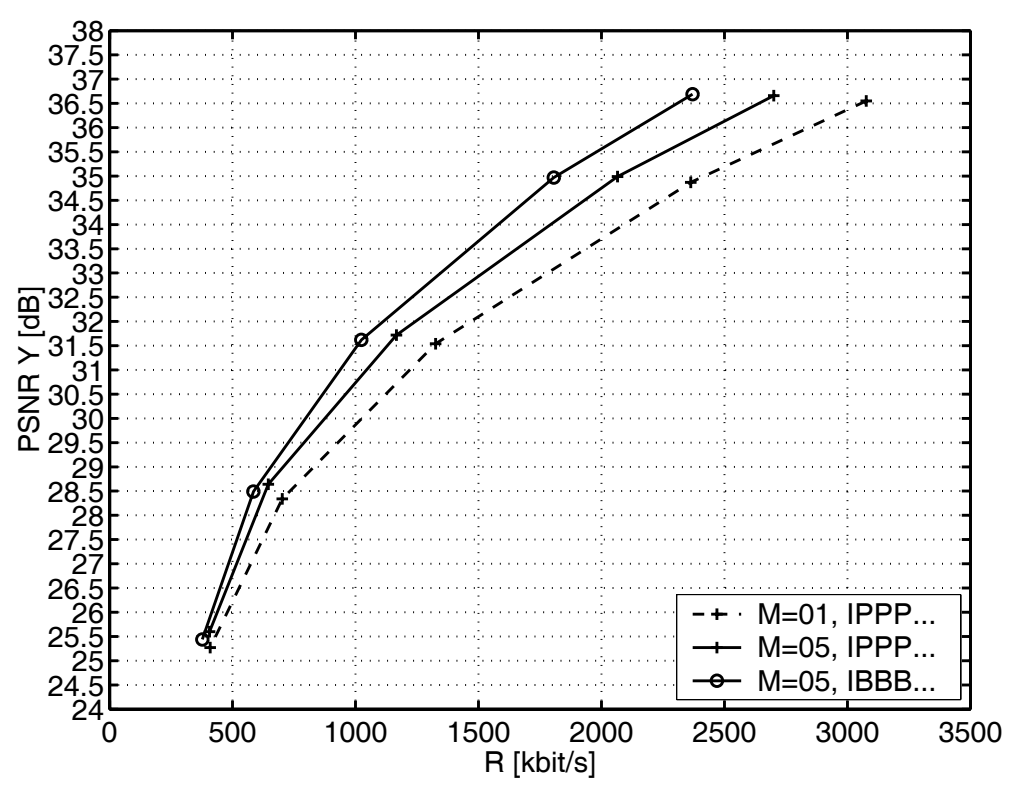

Figure 5.12: PSNR of the luminance signal vs. overall bit-rate for the CIF sequence Mobile 8 Calendar with 30 fps. Generalized B-pictures with forward-only prediction are compared to inter pictures.

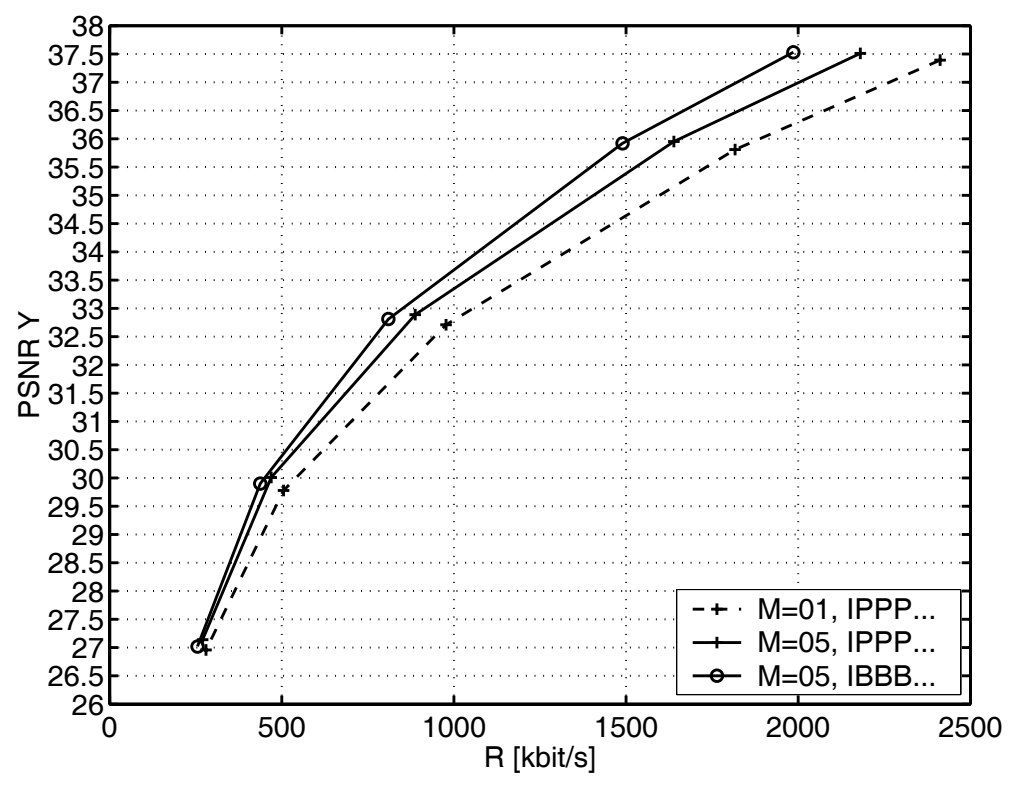

Figure 5.13: PSNR of the luminance signal vs. overall bit-rate for the CIF sequence Tempete with 30 fps. Generalized B-pictures with forward-only prediction are compared to inter pictures. 
Figs. 5.12 and 5.13 depict the average luminance PSNR from reconstructed pictures over the overall bit-rate produced by H.264 inter pictures (IPPP...) and the generalized B-pictures with forward prediction only (IBBB...) for the sequences Mobile \& Calendar and Tempete, respectively. The number of reference pictures is chosen to be $M=1$ and 5 . It can be observed that the gain by generalized B-pictures improves for increasing bit-rate.

\subsubsection{Entropy Coding}

Entropy coding for H.264 B-pictures can be carried out in one of two different ways: universal variable length coding (UVLC) or context-based adaptive binary arithmetic coding (CABAC) [MBW01, MBHW01, SO01]. The UVLC scheme uses only one variable length code to map all syntax elements to binary representations whereas CABAC utilizes context modeling and adaptive arithmetic codes to exploit conditional probabilities and nonstationary symbol statistics [MBHW01]. The simplicity of the UVLC scheme is striking as it demonstrates good compression efficiency at very low computational costs. CABAC with higher computational complexity provides additional bit-rate savings mainly for low and high bit-rates.

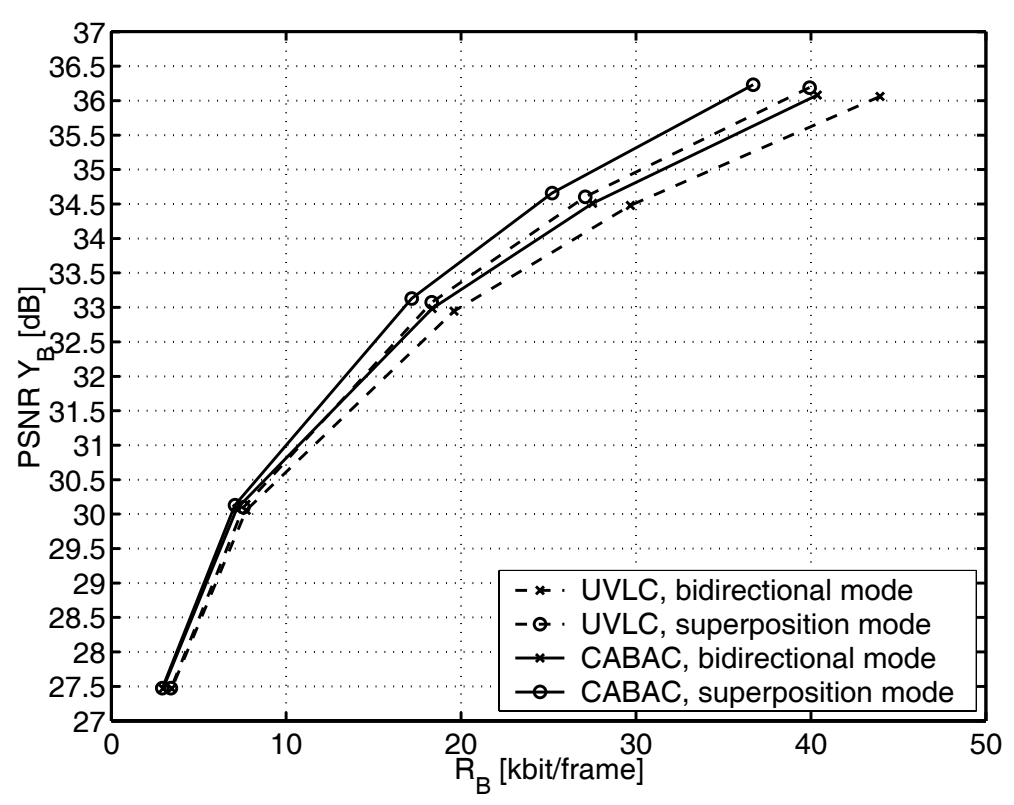

Figure 5.14: PSNR of the B-picture luminance signal vs. B-picture bit-rate for the CIF sequence Mobile $\mathscr{E}$ Calendar with 30 fps. Two B-pictures are inserted after each inter picture. 5 past and 3 future inter pictures are used for predicting each B-picture. $Q P_{B}=Q P_{P}+2$ and $\lambda_{B}=4 w\left(Q P_{P}\right)$. The superposition mode and the bidirectional mode with independent estimation are compared for both entropy coding schemes.

Figs. 5.14 and 5.15 depict the B-picture compression efficiency for the CIF sequences Mobile $\&$ Calendar and Flowergarden, respectively. For motion-compensated prediction, 5 past and 3 future inter pictures are used in all cases. The superposition mode and the bidirectional mode with independent estimation of prediction signals are compared 


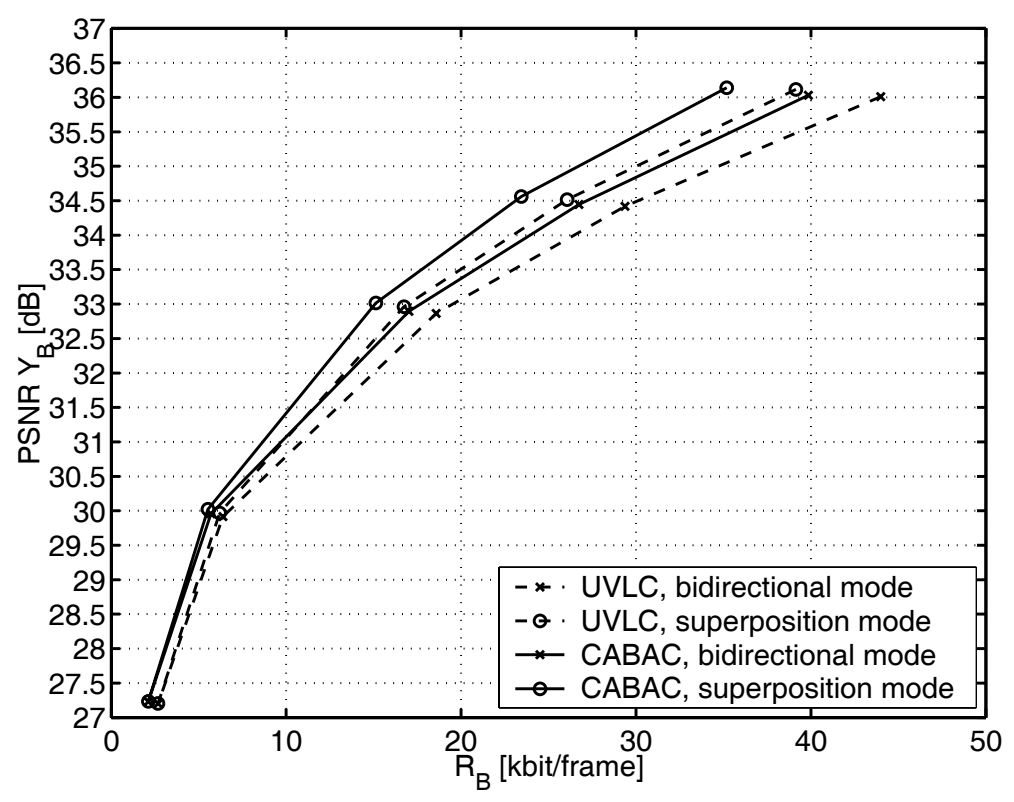

Figure 5.15: PSNR of the B-picture luminance signal vs. B-picture bit-rate for the CIF sequence Flowergarden with 30 fps. Two B-pictures are inserted after each inter picture. 5 past and 3 future inter pictures are used for predicting each B-picture. $Q P_{B}=Q P_{P}+2$ and $\lambda_{B}=4 w\left(Q P_{P}\right)$. The superposition mode and the bidirectional mode with independent estimation are compared for both entropy coding schemes.

for both entropy coding schemes. The PSNR gains by the superposition mode and the CABAC scheme are comparable for the investigated sequences at high bit-rates. When enabling the superposition mode together with CABAC, additive gains can be observed. The superposition mode improves the efficiency of motion-compensated prediction and CABAC optimizes the entropy coding of the utilized syntax elements.

The syntax elements used by the superposition mode can be coded with both the UVLC and the CABAC scheme. When using CABAC for the superposition mode, the context model for the syntax element motion vector data is adapted to superimposed motion. The context model for the motion vector of the first hypothesis captures the motion activity of the spatial neighbors, i.e. the left and top neighboring blocks. The context model for the motion vector of the second hypothesis captures the motion activity of the first hypothesis. The context models for the remaining syntax elements are not altered. The experimental results show that generalizing the bidirectional mode to the superposition mode improves B-picture compression efficiency for both schemes.

\subsection{Encoder Issues}

\subsubsection{Rate Constrained Mode Decision}

The test model TML-9 distinguishes between a low- and high-complexity encoder. For a low-complexity encoder, computationally inexpensive rules for mode decision are recom- 
mended [JP01]. For a high-complexity encoder, the macroblock mode decision is ruled by minimizing the Lagrangian function

$$
J_{1}(\text { Mode } \mid Q P, \lambda)=S S D(\text { Mode } \mid Q P)+\lambda R(\text { Mode } \mid Q P),
$$

where $Q P$ is the macroblock quantizer parameter, and $\lambda$ the Lagrange multiplier for mode decision. Mode indicates the selection from the set of potential coding modes. SSD is the sum of the squared differences between the original block and its reconstruction. It also takes into account the distortion in the chrominance components. $R$ is the number of bits associated with choosing Mode and $Q P$, including the bits for macroblock header, motion information, and all integer transform blocks. The Lagrangian multiplier for $\lambda$ is related to the macroblock quantizer parameter $Q P$ by

$$
\lambda:=w(Q P)=5 \frac{Q P+5}{34-Q P} \exp \left(\frac{Q P}{10}\right) .
$$

Detailed discussions of this relationship can be found in [SW98] and [WG01a]. Experimental results in Section 5.4.4 confirm that this relation should be adapted for B-pictures as specified in the test model TML-9,

$$
\lambda_{B}=4 w\left(Q P_{B}\right)
$$

such that the overall rate-distortion efficiency for the sequence is improved.

Mode decision selects the best mode among all B-picture macroblock modes. In order to reduce encoder complexity, mode decision assumes for all inter modes pre-computed motion vectors which are determined independently by rate-constrained motion estimation.

\subsubsection{Rate Constrained Motion Estimation}

Motion estimation is also performed in a rate-constrained framework. The encoder minimizes the Lagrangian cost function

$$
J_{2}\left(m, r \mid \lambda_{\mathrm{SAD}}, p\right)=S A D(m, r)+\lambda_{\mathrm{SAD}} R(m-p, r)
$$

with the motion vector $m$, the predicted motion vector $p$, the reference frame parameter $r$, and the Lagrange multiplier $\lambda_{\mathrm{SAD}}$ for the $S A D$ distortion measure. The rate term $R$ represents the motion information and the number of bits associated with choosing the reference picture $r$. The rate is estimated by table-lookup using the universal variable length code (UVLC) table, even if the arithmetic entropy coding method is used. For integer-pixel search, $S A D$ is the summed absolute difference between the original luminance signal and the motion-compensated luminance signal. In the sub-pixel refinement search, the Hadamard transform of the difference between the original luminance signal and the motion-compensated luminance signal is calculated and $S A D$ is the sum of the absolute transform coefficients. The Hadamard transform in the sub-pixel search reflects 
the performance of the integer transform on the residual signal such that the expected reconstruction quality rather than the motion-compensated prediction quality is taken into account for the refinement. This favors sub-pixel positions with residuals that are highly correlated for a given summed distortion. The Lagrangian multiplier $\lambda_{\mathrm{SAD}}$ for the $S A D$ distortion measure is related to the Lagrangian multiplier for the $S S D$ measure (5.5) by

$$
\lambda_{\mathrm{SAD}}=\sqrt{\lambda}
$$

Further details as well as block size issues for motion estimation are discussed in [SW98] and [WG01a].

\subsubsection{Rate Constrained Superimposed Motion Estimation}

For the superposition mode, the encoder utilizes rate-constrained superimposed motion estimation. The cost function incorporates the superimposed prediction error of the video signal as well as the bit-rate for two picture reference parameters, two hypotheses types, and the associated motion vectors. Rate-constrained superimposed motion estimation is performed by the hypothesis selection algorithm in Fig. 2.6. This iterative algorithm performs conditional rate-constrained motion estimation and is a computationally feasible solution to the joint estimation problem [WG94] which has to be solved for finding an efficient pair of hypotheses.

The iterative algorithm is initialized with the data of the best macroblock type for multiple reference prediction (initial hypothesis). For two hypotheses, the algorithm continues with:

1. One hypothesis is fixed and conditional rate-constrained motion estimation is applied to the complementary hypothesis such that the superposition costs are minimized.

2. The complementary hypothesis is fixed and the first hypothesis is optimized.

The two steps (= one iteration) are repeated until convergence. For the current hypothesis, conditional rate-constrained motion estimation determines the conditional optimal picture reference parameter, hypothesis type, and associated motion vectors. For the conditional optimal motion vectors, an integer-pel accurate estimate is refined to sub-pel accuracy.

Fig. 5.16 shows the average number of iterations for superimposed motion estimation with 5 reference pictures over the quantization parameter. On average, it takes about 2 iterations to achieve a Lagrangian cost smaller than $0.5 \%$ relative to the cost in the previous iteration. The algorithm converges faster for higher quantization parameter values.

Given the best single hypothesis for motion-compensated prediction (best inter mode) and the best hypothesis pair for superimposed prediction, the resulting prediction errors are transform coded to compute the Lagrangian costs for the mode decision.

Superimposed motion-compensated prediction improves the prediction signal by allocating more bits to the side-information associated with the motion-compensating predictor. But the encoding of the prediction error and its associated bit-rate also determines 


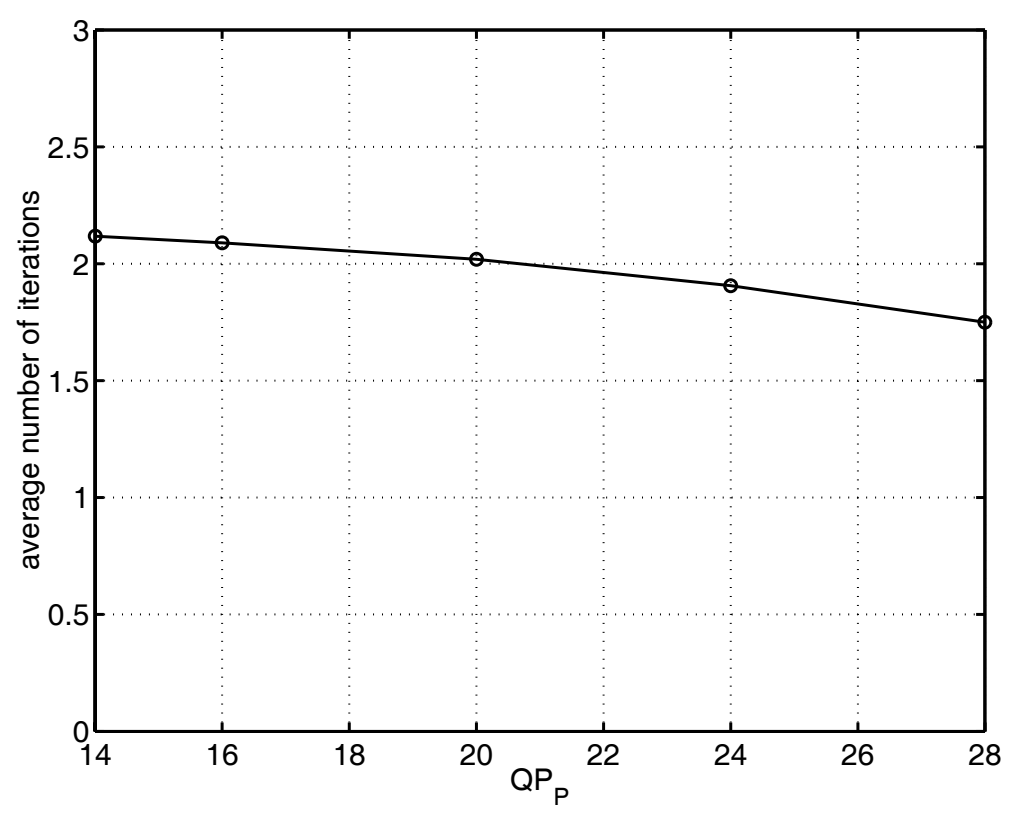

Figure 5.16: Average number of iterations for superimposed motion estimation vs. quantization parameter for the CIF sequence Mobile \& Calendar with $30 \mathrm{fps}$ and $M=5$ reference pictures.

the quality of the reconstructed macroblock. A joint optimization of superimposed motion estimation and prediction error coding is far too demanding. But superimposed motion estimation independent of prediction error encoding is an efficient and practical solution if rate-constrained superimposed motion estimation is applied.

It turns out that the superposition mode is not necessarily the best one for each macroblock. Therefore, the rate-distortion optimal mode selection is a very important tool to decide whether a macroblock should be predicted with one or two hypotheses.

Fig. 5.17 shows the relative occurrence of the superposition mode in generalized Bpictures over the quantization parameter for the CIF sequence Mobile \& Calendar. 5 past and 3 future reference pictures are used. Results for both entropy coding schemes are plotted. For high bit-rates (small quantization parameters), the superposition mode exceeds a relative occurrence of $50 \%$ among all B-picture coding modes. For low bitrates (large quantization parameters), the superposition mode is selected infrequently and, consequently, the improvement in coding efficiency is very small. In addition, the relative occurrence is slightly larger for the CABAC entropy coding scheme since the more efficient CABAC scheme somewhat relieves the rate constraint imposed on the side-information.

\subsubsection{Improving Overall Rate-Distortion Performance}

When B-pictures are considered to be an enhancement layer in a scalable representation, they are predicted from reference pictures that are provided by the base layer. Consequently, the quality of the base layer influences the rate-distortion trade-off for B-pictures in the enhancement layer. Experimental results show that the relationship between quantization and Lagrange parameter for mode decision $\lambda=w(Q P)$ should be adapted [SW01]. 


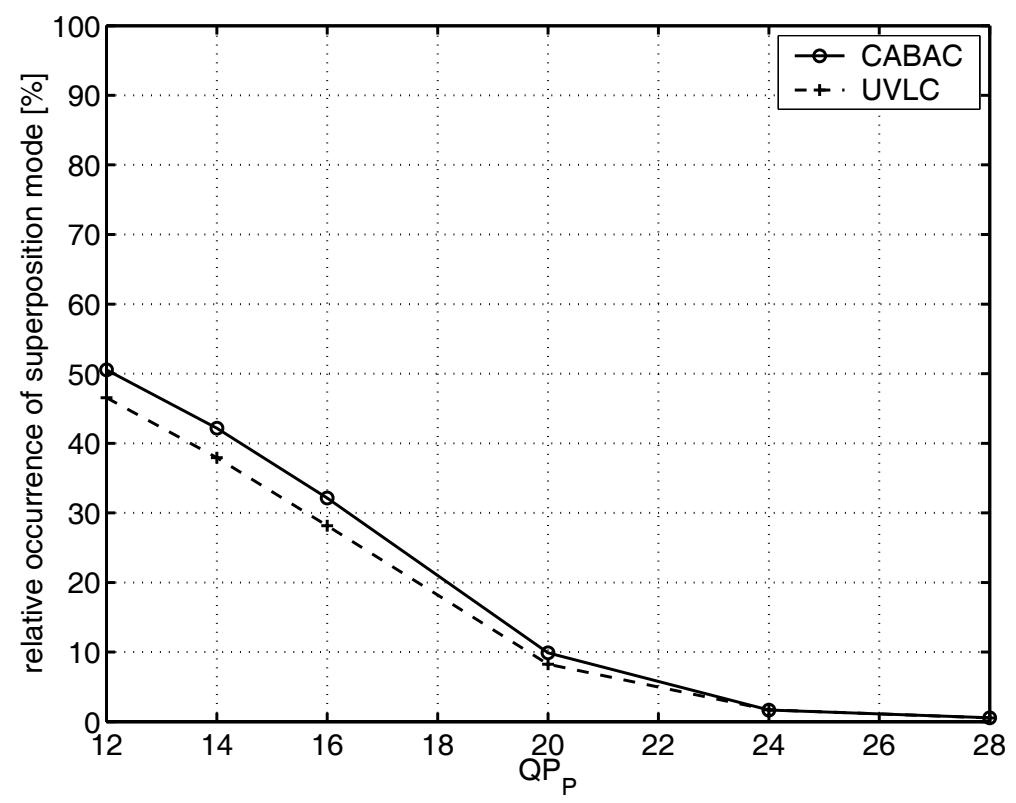

Figure 5.17: Relative occurrence of the superposition mode in B-pictures vs. quantization parameter for the CIF sequence Mobile $\&$ Calendar with 30 fps. 5 past and 3 future reference pictures are used. $Q P_{B}=Q P_{P}+2$.

The following experimental results are obtained with the test model software TML-9, i.e., with bidirectional prediction and independent estimation of forward and backward prediction parameters.

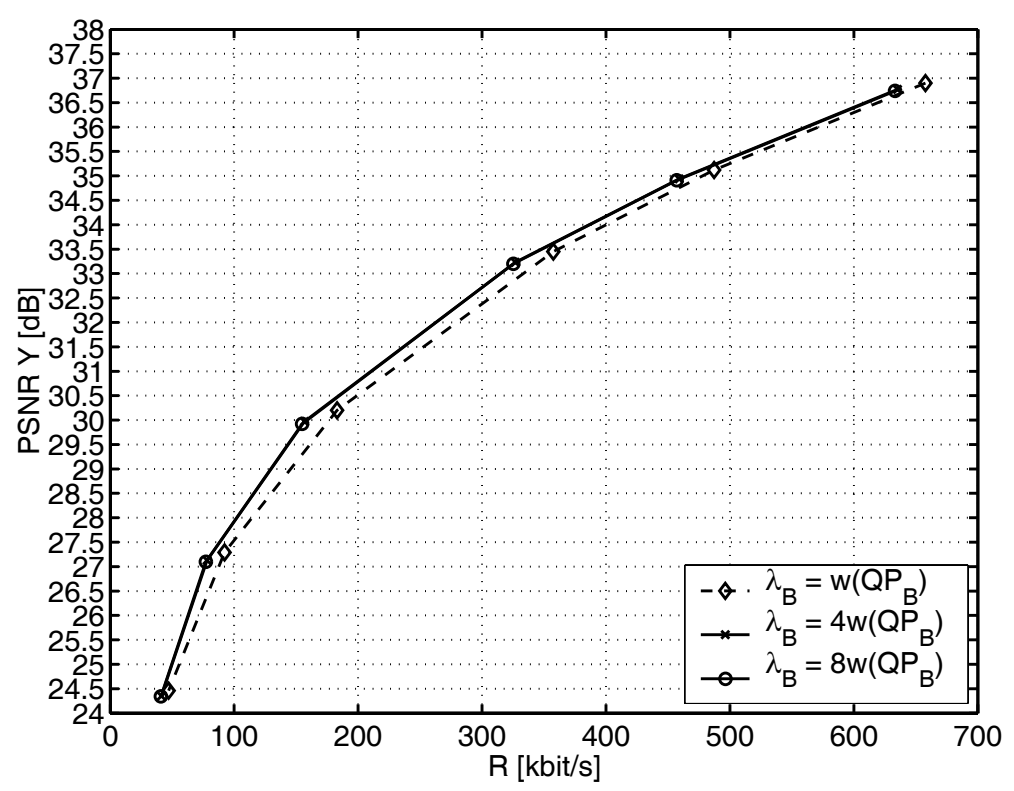

Figure 5.18: PSNR of the luminance signal vs. overall bit-rate for the QCIF sequence Mobile $\mathcal{E}$ Calendar with $30 \mathrm{fps}$. Two B-pictures are inserted and the influence of the $\lambda_{B}-Q P_{B}$ relationship on the overall compression efficiency is investigated. 
Fig. 5.18 shows the PSNR of the luminance signal vs. overall bit-rate for the QCIF sequence Mobile $\&$ Calendar with 30 fps. Three different $\lambda-Q P$ dependencies are depicted. The worst compression efficiency is obtained with $\lambda_{B}=w\left(Q P_{B}\right)$. The cases $\lambda_{B}=4 w\left(Q P_{B}\right)$ and $\lambda_{B}=8 w\left(Q P_{B}\right)$ demonstrate superior efficiency for low bit-rates. The scaling of the dependency alters the bit-rate penalty for all B-picture coding modes such that the overall compression efficiency is improved. The factor 4 is suggested in the current test model description.

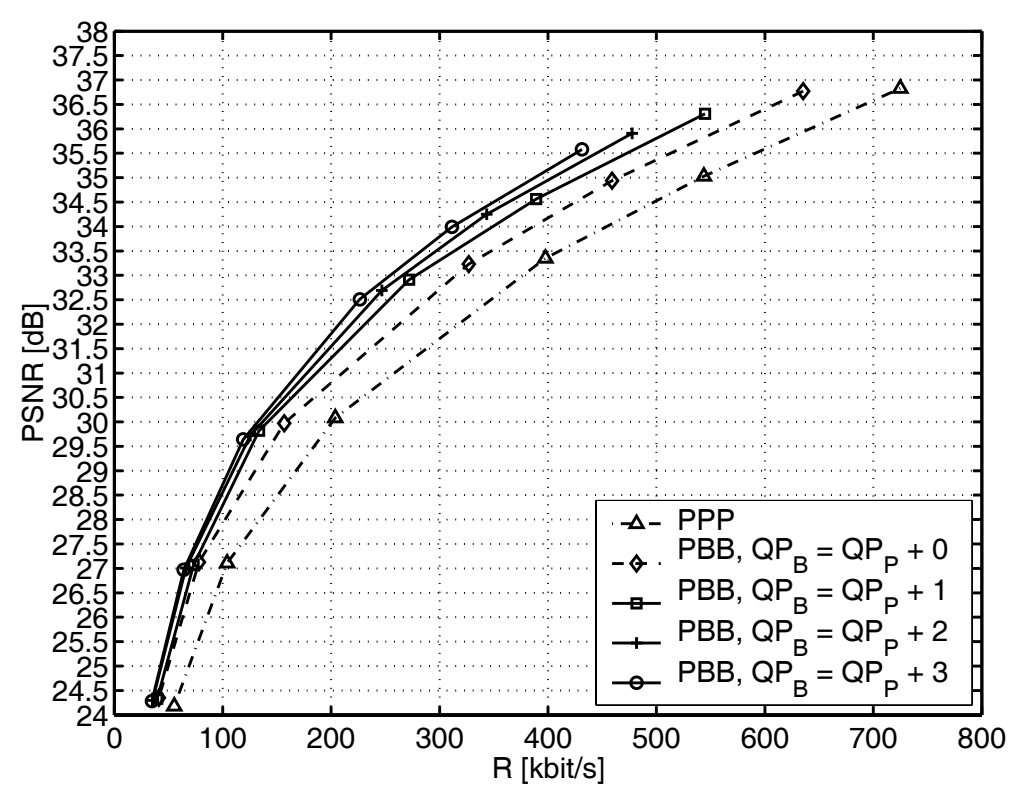

Figure 5.19: PSNR of the luminance signal vs. overall bit-rate for the QCIF sequence Mobile $\mathbb{E}$ Calendar with $30 \mathrm{fps}$. Two B-pictures are inserted and the influence of the B-picture quantization parameter $Q P_{B}$ on the overall compression efficiency is investigated for $\lambda_{B}=4 w\left(Q P_{B}\right)$.

Further experiments show that not only the relationship between quantization and Lagrange parameter for mode decision has to be adapted for B-pictures but also the PSNR of the enhancement layer should be lowered in comparison to the base layer to improve overall compression efficiency [SW01]. Fig. 5.19 depicts also the PSNR of the luminance signal vs. overall bit-rate for the QCIF sequence Mobile $\&$ Calendar with $30 \mathrm{fps}$. The plot compares the compression efficiency of various layered bit-streams with two inserted Bpictures. The quantization parameters of inter and B-pictures differ by a constant offset. For comparison, the efficiency of the single layer bit-stream is provided. Increasing the quantization parameter for B-pictures, that is, lowering their relative PSNR, improves the overall compression efficiency of the sequence.

Fig. 5.20 shows the PSNR of the luminance signal for individual pictures of the sequence Mobile $\&$ Calendar encoded with $Q P_{P}=14$. The PSNR of the B-pictures encoded with an increment of 2 is significantly lower compared to the case with identical quantization parameter in both layers. The compression efficiency of the sequence increases by lowering the relative PSNR of the enhancement layer. For the investigated sequence, the average PSNR efficiency increases by almost $1 \mathrm{~dB}$ (see Fig. 5.19), whereas the PSNR 


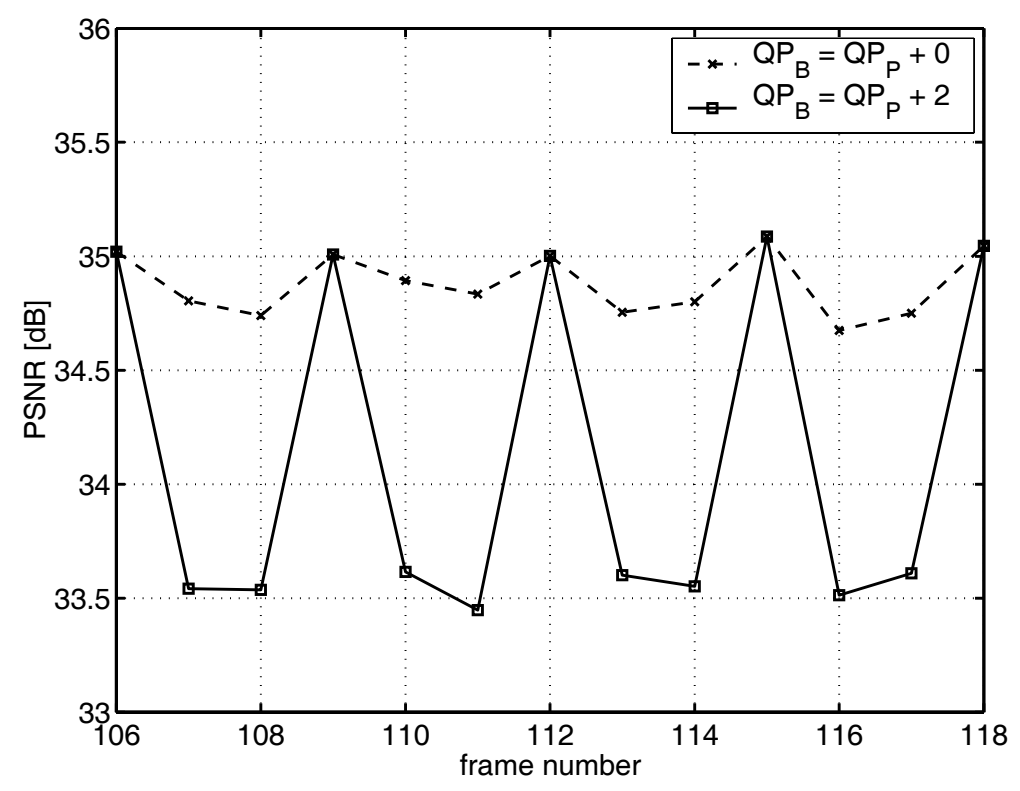

Figure 5.20: PSNR of the luminance signal for individual pictures. Two B-pictures are inserted. The B-picture quantization parameter $Q P_{B}$ is incremented by 2 and the B-picture Lagrange parameter $\lambda_{B}=4 w\left(Q P_{B}\right) \cdot Q P_{P}=14$.

of individual B-pictures drops by more than $1 \mathrm{~dB}$. In this case, higher average PSNR with temporal fluctuations is compared to lower average PSNR with less fluctuations for a given bit-rate.

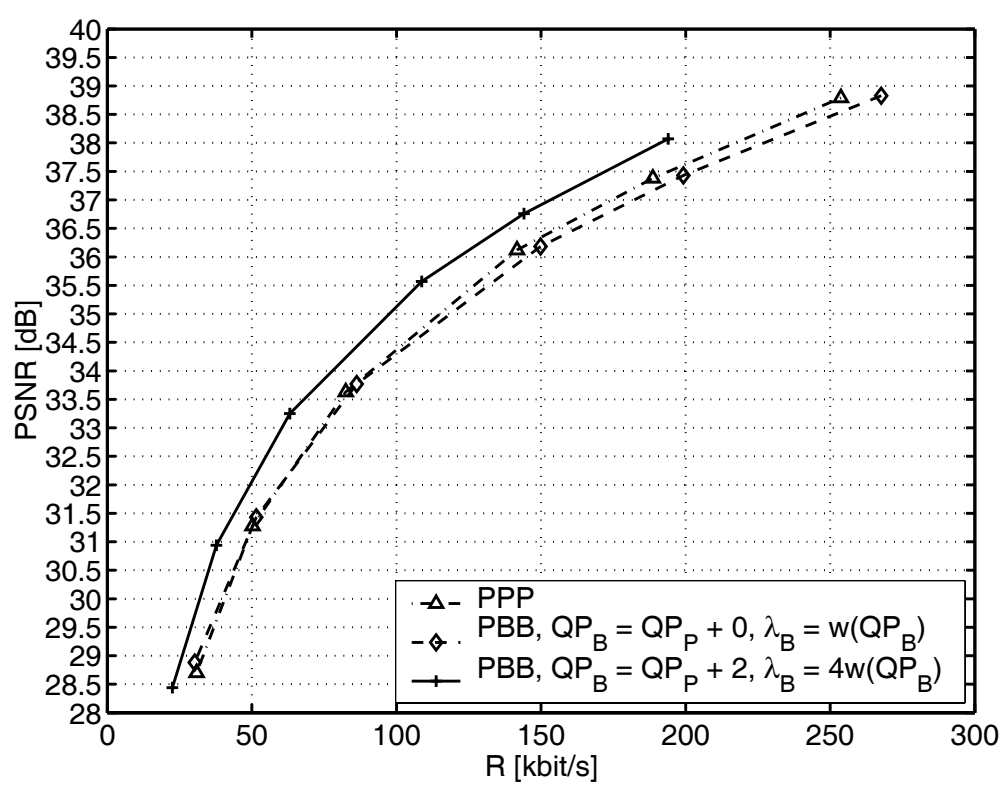

Figure 5.21: PSNR of the luminance signal vs. overall bit-rate for the QCIF sequence Foreman with $30 \mathrm{fps}$. When replacing two inter pictures by B-pictures, the values $Q P_{B}$ and $\lambda_{B}$ have to be adapted for best compression efficiency. Keeping the inter picture values may lower the efficiency. 
Fig. 5.21 shows the PSNR of the luminance signal vs. overall bit-rate for the QCIF sequence Foreman with 30 fps. The depicted results demonstrate that without adapting the quantization parameter and the $\lambda-Q P$ dependency for B-pictures, we observe a degradation in compression efficiency if two inter pictures are replaced by B-pictures, whereas the adaptation improves the PSNR by about $0.5 \mathrm{~dB}$ for a given bit-rate.

\subsection{Conclusions}

This chapter discusses B-pictures in the context of the draft H.264 video compression standard. We focus on reference picture selection and linearly combined motion-compensated prediction signals. We show that bidirectional prediction only partially exploits the efficiency of combined prediction signals whereas superimposed prediction allows a more general form of B-pictures. The general concept of linearly combined prediction signals chosen from an arbitrary set of reference pictures further improves the H.264 test model TML-9 which is used in this chapter.

We outline H.264 macroblock prediction modes for B-pictures, classify them into four groups and compare their efficiency in terms of rate-distortion performance. When investigating superimposed prediction, we show that bidirectional prediction is a special case of this concept. Superimposed prediction allows also two combined forward prediction signals. Experimental results show that this case is also advantageous in terms of compression efficiency. The draft H.264 video compression standard offers improved entropy coding by context-based adaptive binary arithmetic coding. Simulations show that the gains by superimposed prediction and arithmetic coding are additive. B-pictures establish an enhancement layer and are predicted from reference pictures that are provided by the base layer. The quality of the base layer influences the rate-distortion trade-off for B-pictures. We demonstrate how the quality of the B-pictures should be reduced in order to improve the overall rate-distortion performance of the scalable representation.

Conceptually, we differentiate between picture reference selection and linearly combined prediction signals. This distinction is reflected in the term Generalized B-Pictures. The feature of reference picture selection has been improved significantly when compared to existing video compression standards. But the draft H.264 video compression standard has been extended recently to support the features of linearly combined prediction signals that are described in this chapter.

Towards a generalized picture type, a desirable definition would make a distinction whether only past reference pictures or also future reference pictures are used for prediction. If both past and future reference pictures are available, this generalized picture type would utilize the direct and superposition mode, whereas if only past reference frames are available, this generalized picture type would replace the direct mode by the copy mode. 


\section{Motion Compensation for Groups of Pictures}

\subsection{Introduction}

So far, we discussed video coding schemes that utilize inter-frame methods with motioncompensated prediction (MCP) for efficient compression. Such compression schemes require sequential processing of video signals which makes it difficult to achieve efficient embedded representations of video sequences. This chapter investigates the efficiency of motion-compensated 3D transform coding, a compression scheme that employs a motioncompensated transform for groups of pictures. We investigate this coding scheme experimentally and theoretically. The practical coding scheme employs in temporal direction a motion-compensated subband decomposition for each group of pictures. We also compare the experimental results to that of a predictive video codec with motion compensation and comparable complexity. The theoretical investigation models this motioncompensated subband coding scheme for a group of $K$ pictures with a signal model for $K$ motion-compensated pictures that are decorrelated by a linear transform. We utilize the Karhunen-Loeve Transform to obtain theoretical performance bounds at high bit-rates and compare to both optimum intra-frame coding of individual motion-compensated pictures and motion-compensated predictive coding.

The outline of this chapter is as follows: Section 6.2 discusses the motion-compensated subband coding scheme with a motion-compensated lifted Haar wavelet and a motioncompensated lifted 5/3 wavelet. Experimental results and comparisons for several test sequences are presented. The theoretical signal model is developed in Section 6.3. The motion-compensated wavelet kernels of the practical coding schemes are generalized to obtain theoretical performance bounds.

\subsection{Coding Scheme}

Applying a linear transform in temporal direction of a video sequence is not very efficient if significant motion is prevalent. However, a combination of a linear transform and motion compensation seems promising for efficient compression. For wavelet transforms, the so called Lifting Scheme [Swe98] can be used to construct the kernels. A two-channel decomposition can be achieved with a sequence of prediction and update steps that form a ladder structure. The advantage is that this lifting structure is able to map integers to integers without requiring invertible lifting steps. Further, motion compensation can 
be incorporated into the prediction and update steps as proposed in [ST01]. The fact that the lifting structure is invertible without requiring invertible lifting steps makes this approach feasible. We cannot count on motion compensation to be invertible in general. If it is invertible, this motion-compensated wavelet transform based on lifting permits a linear transform along the motion trajectories in a video sequence.

In the following, we investigate coding schemes that process video sequences in groups of $K$ pictures (GOP). First, we decompose each GOP in temporal direction. The dyadic decomposition utilizes a motion-compensated wavelet which will be discussed later in more detail. The temporal transform provides $K$ output pictures that are intra-frame encoded. In order to allow a comparison to a basic predictive coder with motion compensation, we utilize for the intra-frame coder a $8 \times 8$ DCT with run-length coding. If we employ a Haar wavelet and set the motion vectors to zero, the dyadic decomposition will be an orthonormal transform. Therefore, we select the same quantizer step-size for all $K$ intra-frame encoder. The motion information that is required for the motion-compensated wavelet transform is estimated in each decomposition level depending on the results of the lower level. Further, we employ half-pel accurate motion compensation with bi-linear interpolation.

\subsubsection{Motion-Compensated Lifted Haar Wavelet}

First, we discuss the lifting scheme with motion compensation for the Haar wavelet [ST01].

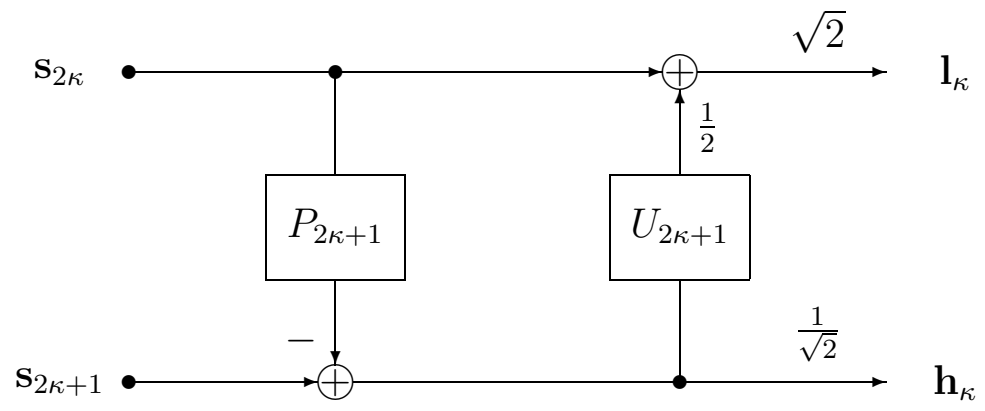

Figure 6.1: Haar transform with motion-compensated lifting steps. Both steps, prediction $P$ and update $U$, utilize block-based motion compensation.

Fig. 6.1 depicts a Haar transform with motion-compensated lifting steps. The even frames of the video sequence $\mathbf{s}_{2 \kappa}$ are used to predict the odd frames $\mathbf{s}_{2 \kappa+1}$ (prediction step $P)$. The prediction step is followed by an update step $U$. If the motion field between the even and odd frames is invertible, the corresponding motion vectors in the update and prediction steps sum to zero. We use a block-size of $16 \times 16$ and half-pel accurate motion compensation in the prediction step and select the motion vectors such that they minimize the squared error in the high-band $\mathbf{h}_{\kappa}$. In general, this block-motion field is not invertible but we still utilize the negative motion vectors for the update step as an approximation. Additional scaling factors in low- and high-band are necessary to normalize the transform. 


\subsubsection{Motion-Compensated Lifted 5/3 Wavelet}

The Haar wavelet is a short filter and provides limited coding gain. We expect better coding efficiency with longer wavelet kernels. In the following, we discuss the lifted 5/3 wavelet kernel with motion compensation [ST01].

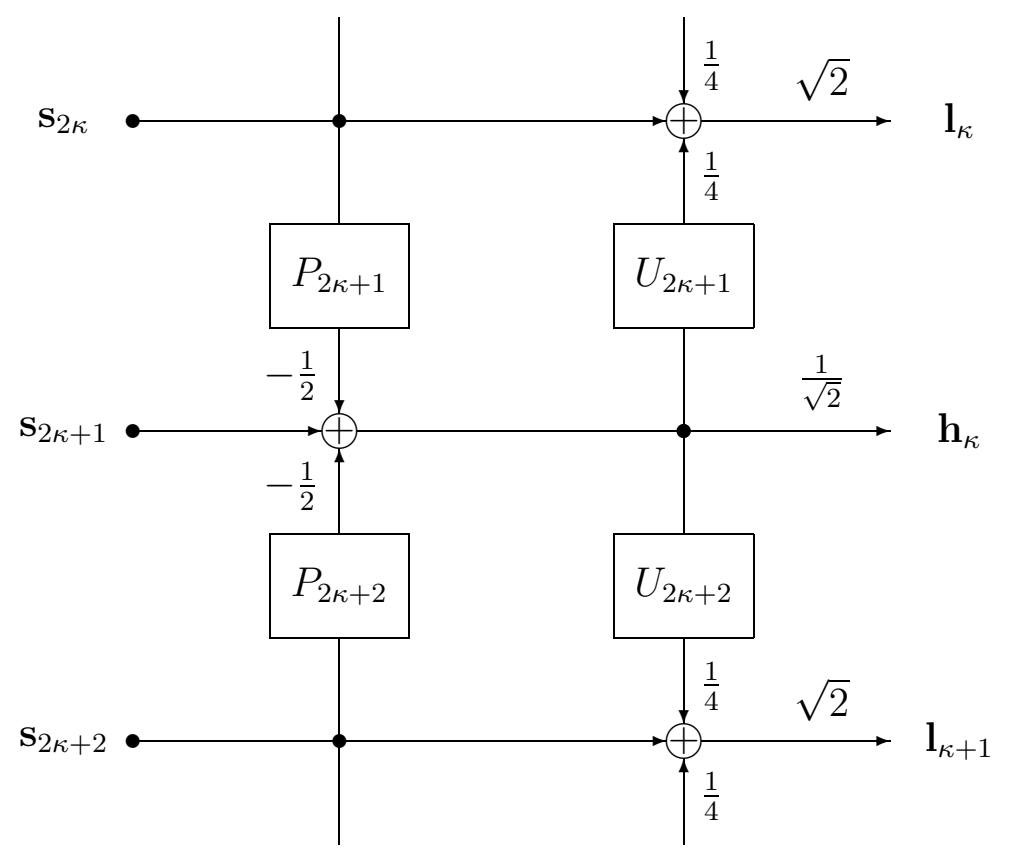

Figure 6.2: Lifted 5/3 wavelet with motion compensation.

Fig. 6.2 depicts the $5 / 3$ transform with motion-compensated lifting steps. Similar to the Haar transform, the update steps $U_{2 \kappa+1}$ use the negative motion vectors of the corresponding prediction steps. But for this transform, the odd frames are predicted by a linear combination of two displaced neighboring even frames. Again, we use a block-size of $16 \times 16$ and half-pel accurate motion compensation in the prediction steps and choose the motion vectors for $P_{2 \kappa+1}$ and $P_{2 \kappa+2}$ such that they minimize the squared error in the high-band $\mathbf{h}_{\kappa}$. The corresponding update steps $U_{2 \kappa+2}$ use also the negative motion vectors of the corresponding prediction steps.

\subsubsection{Experimental Results}

For the experiments, we subdivide the QCIF sequences Mother $\&$ Daughter, Container Ship, Salesman, Mobile \& Calendar, Foreman, News, and Car Phone, each with 288 frames at $30 \mathrm{fps}$, into groups of $K$ pictures. We decompose the GOPs independently and in the case of the $5 / 3$ wavelet, we refer back to the first picture in the GOP when the GOP terminates. We will justify later, why we choose this cyclic extension to handle the GOP boundaries.

Figs. 6.3, 6.4, 6.5, 6.6, 6.7, 6.8, and 6.9 show luminance PSNR over the total bit-rate for the test sequences encoded for groups of $K=2,8,16$, and 32 pictures with the Haar kernel and for groups of $K=32$ with the $5 / 3$ kernel. 


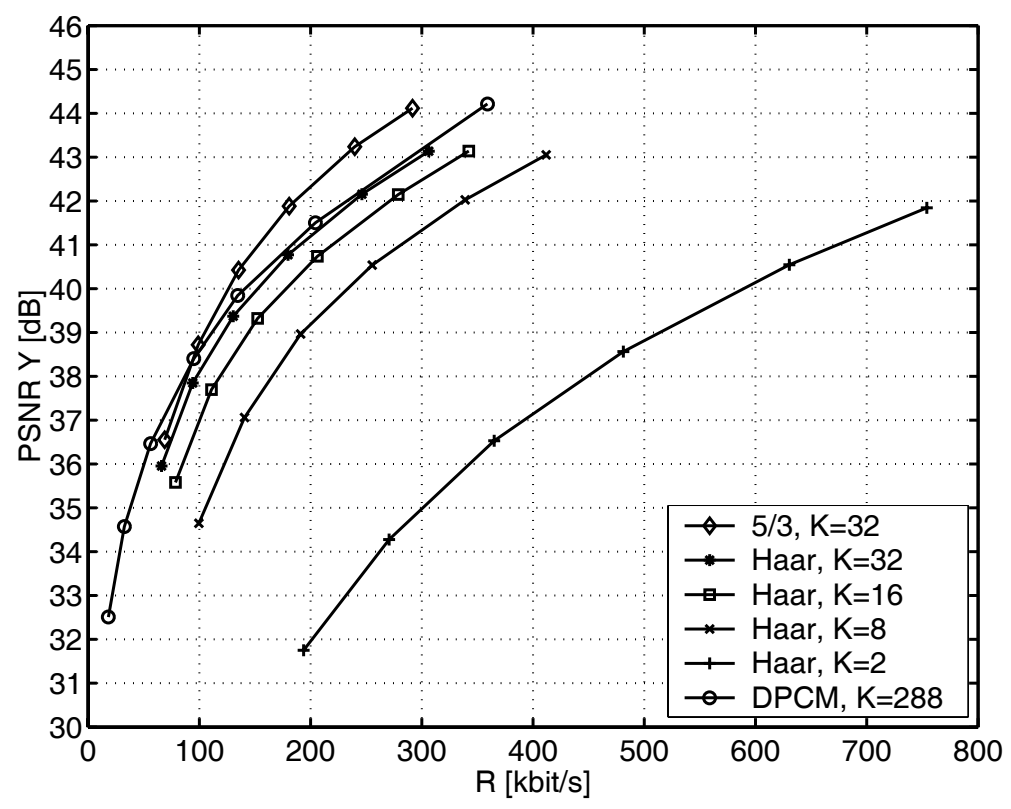

Figure 6.3: Luminance PSNR vs. total bit-rate for the QCIF sequence Mother $\&$ Daughter at 30 fps. A dyadic decomposition is used to encode groups of $K=2,8,16$, and 32 pictures with the Haar kernel, and $K=32$ with the $5 / 3$ kernel. Results for a basic predictive video codec with 287 inter-frames are given for reference.

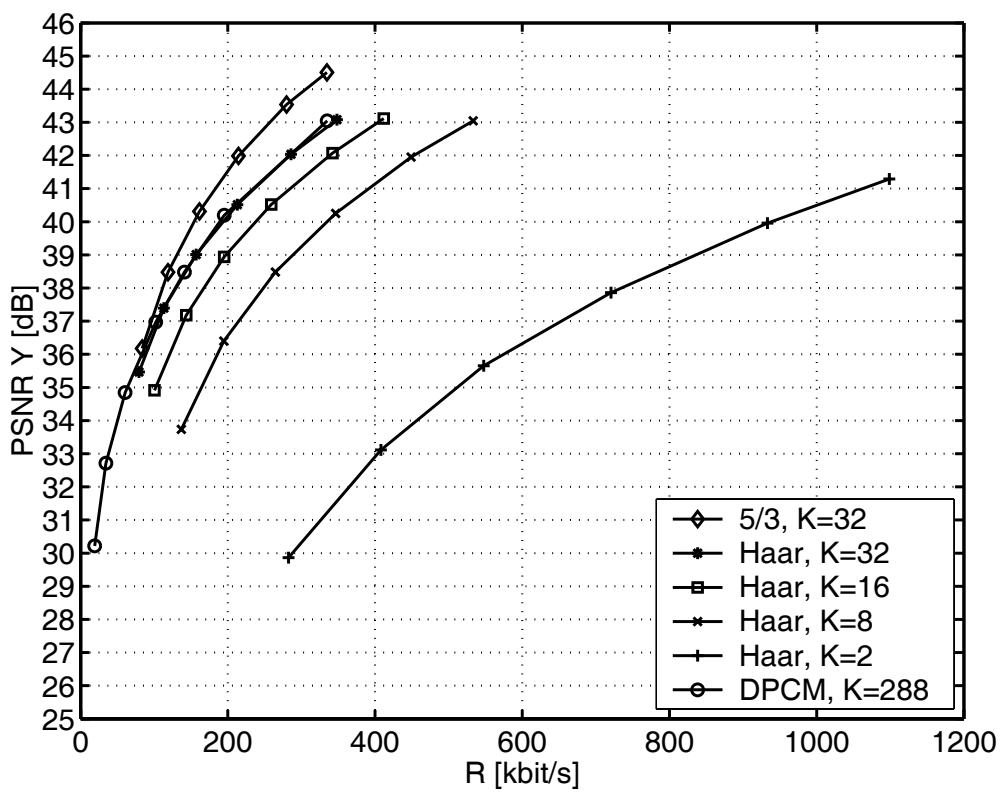

Figure 6.4: Luminance PSNR vs. total bit-rate for the QCIF sequence Container Ship at 30 fps. A dyadic decomposition is used to encode groups of $K=2,8,16$, and 32 pictures with the Haar kernel, and $K=32$ with the $5 / 3$ kernel. Results for a basic predictive video codec with 287 inter-frames are given for reference. 


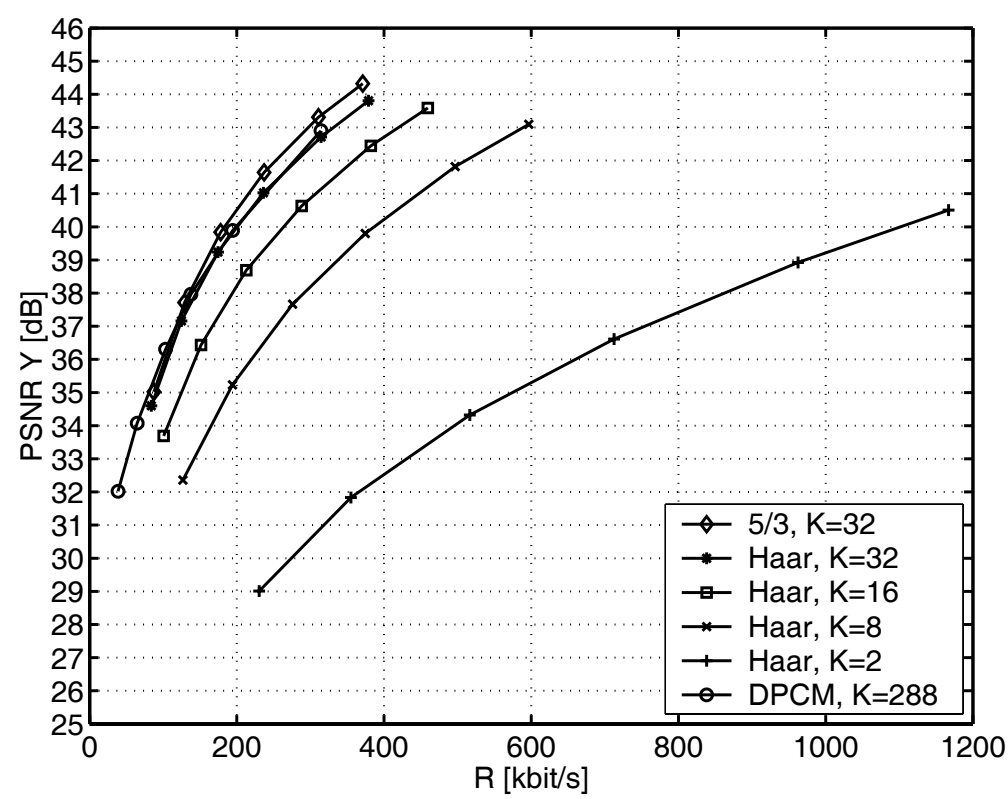

Figure 6.5: Luminance PSNR vs. total bit-rate for the QCIF sequence Salesman at 30 fps. A dyadic decomposition is used to encode groups of $K=2,8,16$, and 32 pictures with the Haar kernel, and $K=32$ with the $5 / 3$ kernel. Results for a basic predictive video codec with 287 inter-frames are given for reference.

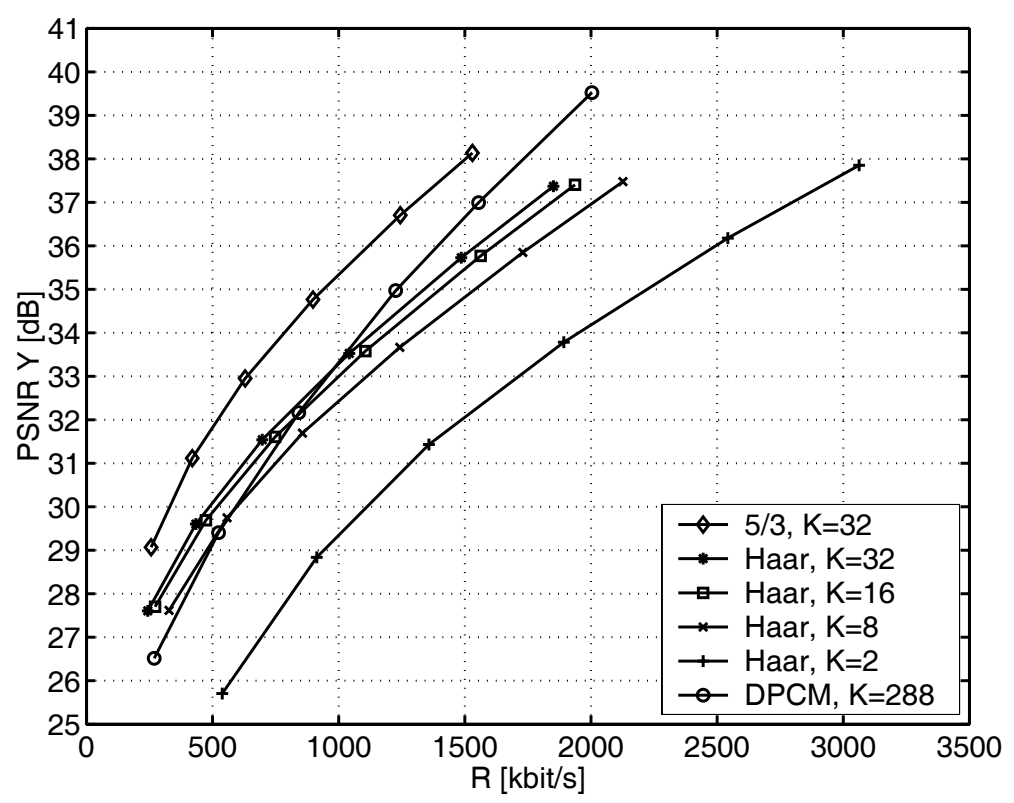

Figure 6.6: Luminance PSNR vs. total bit-rate for the QCIF sequence Mobile \& Calendar at 30 fps. A dyadic decomposition is used to encode groups of $K=2,8,16$, and 32 pictures with the Haar kernel, and $K=32$ with the $5 / 3$ kernel. Results for a basic predictive video codec with 287 inter-frames are given for reference. 


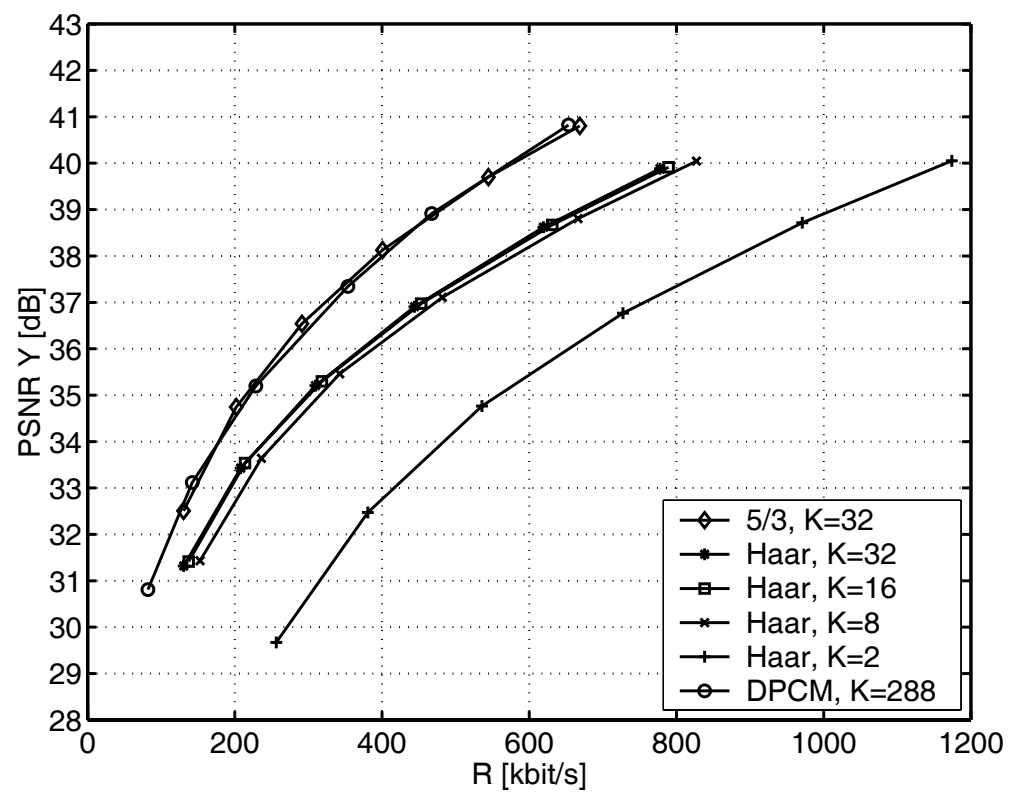

Figure 6.7: Luminance PSNR vs. total bit-rate for the QCIF sequence Foreman at 30 fps. A dyadic decomposition is used to encode groups of $K=2,8,16$, and 32 pictures with the Haar kernel, and $K=32$ with the $5 / 3$ kernel. Results for a basic predictive video codec with 287 inter-frames are given for reference.

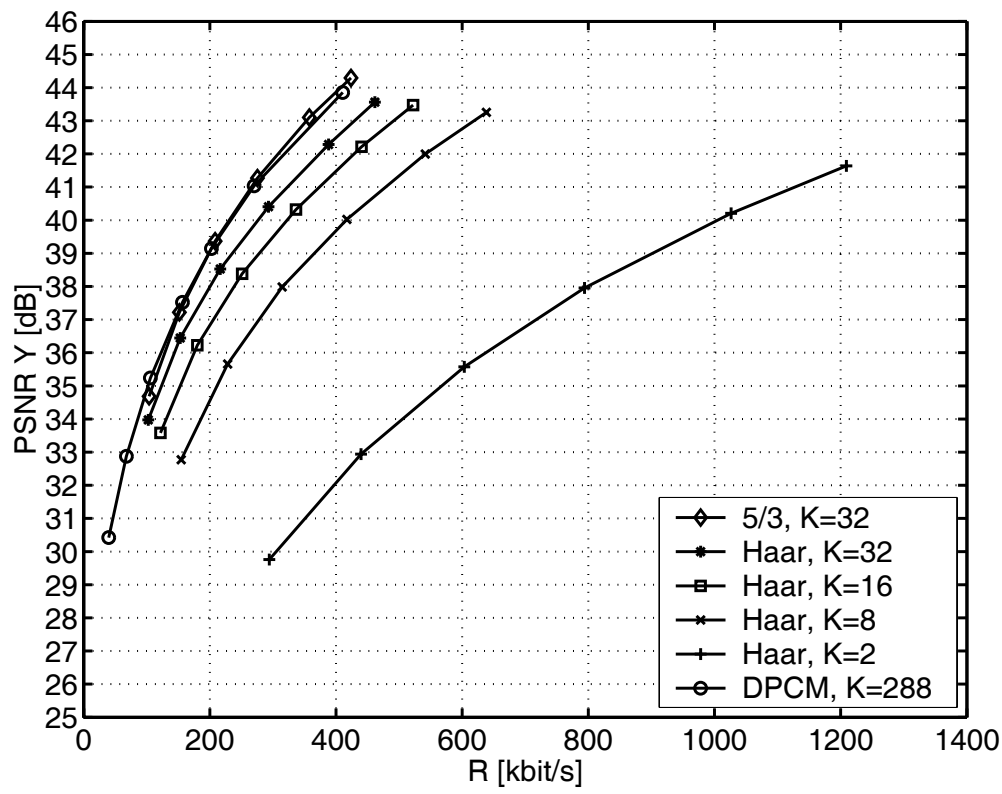

Figure 6.8: Luminance PSNR vs. total bit-rate for the QCIF sequence News at 30 fps. A dyadic decomposition is used to encode groups of $K=2,8,16$, and 32 pictures with the Haar kernel, and $K=32$ with the $5 / 3$ kernel. Results for a basic predictive video codec with 287 inter-frames are given for reference. 


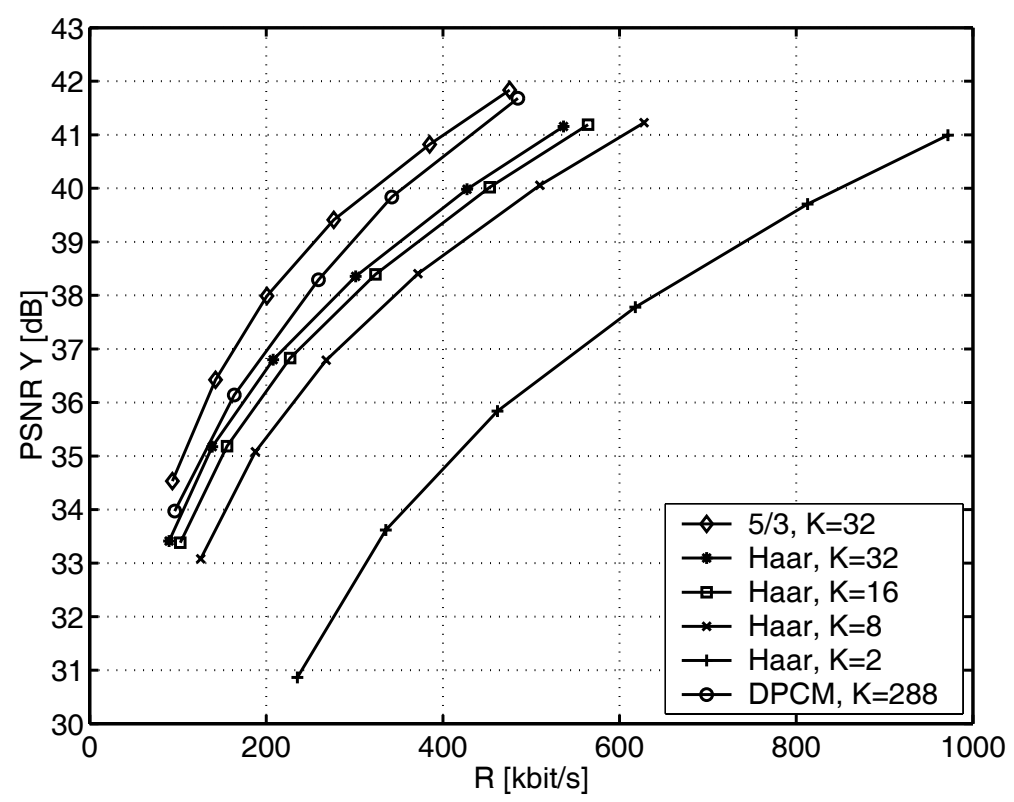

Figure 6.9: Luminance PSNR vs. total bit-rate for the QCIF sequence Car Phone at $30 \mathrm{fps}$. A dyadic decomposition is used to encode groups of $K=2,8,16$, and 32 pictures with the Haar kernel, and $K=32$ with the $5 / 3$ kernel. Results for a basic predictive video codec with 287 inter-frames are given for reference.
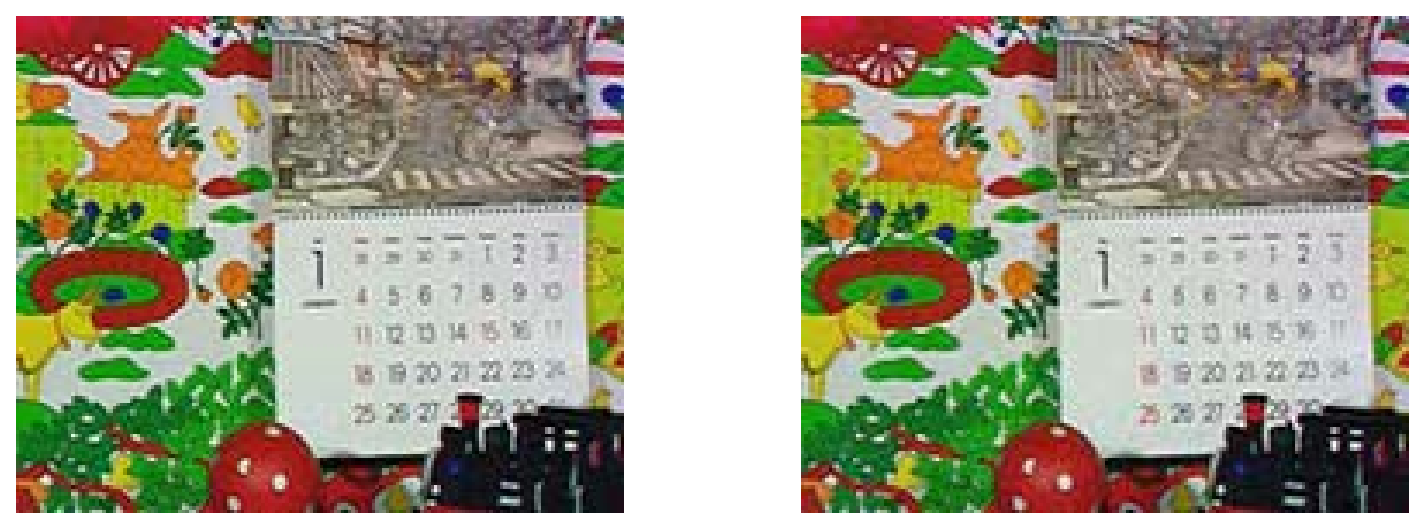

Figure 6.10: Frame 28 of the sequence Mobile $\&$ Calendar encoded with the motion-compensated $5 / 3$ wavelet coder (left) and the motion-compensated predictive coder (right). The left frame has a PSNR of $35.1 \mathrm{~dB}$, the right frame $32.2 \mathrm{~dB}$. The wavelet coder achieves an average PSNR of $34.8 \mathrm{~dB}$ at $890 \mathrm{kbit} / \mathrm{s}$, the predictive coder an average PSNR of $32.2 \mathrm{~dB}$ at $840 \mathrm{kbit} / \mathrm{s}$.

We observe that the bit-rate savings with the Haar kernel diminish very quickly as the GOP size approaches 32 pictures. Note also that the $5 / 3$ decomposition with a GOP size of 32 outperforms the Haar decomposition with a GOP size of 32 . For the sequences Mother $\&$ Daughter, Container Ship, and Salesman, the Haar wavelet coding scheme with $K=32$ performs similar to a comparable basic predictive video codec (intra- and interframes, $16 \times 16$ block-motion compensation, half-pel accuracy, previous reference picture only, and $8 \times 8$ DCT) with a very large GOP size. Please note that for Mobile $\&$ Calendar 
at lower bit-rates the Haar wavelet coding scheme outperforms the predictive video codec. The 5/3 decomposition with a GOP size of 32 outperforms not only the corresponding Haar decomposition but also the basic predictive video coding scheme with a GOP size of $K=288$. See also Fig. 6.10.

For the sequences Foreman, News, and Car Phone, the 5/3 wavelet coding scheme performs comparable or slightly better than the predictive video codec. These sequences contain inhomogeneous motion and we suspect that the use of negative motion vectors in the update step permits only an insufficient approximation.

Further, we investigate the behavior of the coding scheme for the cases that the frames are degraded by additive noise. For that, we generate the sequence Noisy Foreman by repeating 32 times the first frame of the sequence Foreman and adding statistically independent white Gaussian noise of variance 25. As we investigate the residual noise only, this sequence contains no motion. Predictive coding with motion compensation is not capable of predicting the additive noise in the current frame. In fact, prediction doubles the noise variance in the residual signal and we expect that predictive coding performs inferior.

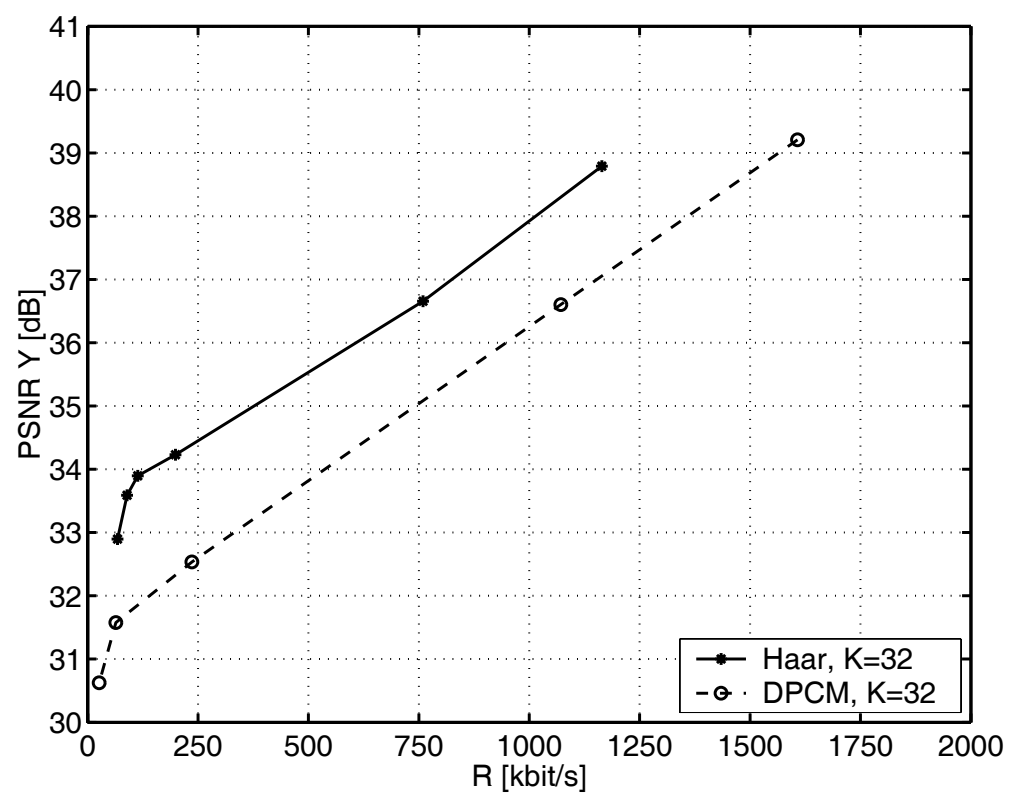

Figure 6.11: Luminance PSNR vs. total bit-rate for the QCIF sequence Noisy Foreman at 30 fps. A dyadic decomposition is used to encode groups of $K=32$ pictures with the motioncompensated Haar wavelet. Results for a basic predictive video codec with 31 inter-frames are given for reference.

Fig. 6.11 shows luminance PSNR over the total bit-rate for the sequence Noisy Foreman. The coding scheme with the Haar wavelet kernel and a dyadic decomposition of 32 pictures is compared to the predictive coding scheme. We observe that the wavelet coding scheme outperforms the predictive coding scheme by approximately $2 \mathrm{~dB}$. The predictive coding scheme is inferior as the statistically independent noise in the current frame cannot be predicted from the previous frame. 
Finally, we discuss briefly our GOP boundary handling for the $5 / 3$ wavelet. As we encode the GOPs in the sequence independently, we have to solve the boundary problem for the $5 / 3$ wavelet. For the discussion, we consider cyclic and symmetric extensions at the GOP boundary. Note that a GOP begins with the even picture $\mathbf{s}_{0}$ and terminates always with an odd picture. When the GOP terminates, the cyclic extension refers back to the first picture $\mathbf{s}_{0}$, and the symmetric extension uses the last even picture in the GOP twice as a reference. In the case of the cyclic extension, the terminal update step modifies the first picture $\mathbf{s}_{0}$, and in the case of the symmetric extension, the last even picture in the GOP is updated twice. We implemented both extensions for an experimental comparison of the rate-distortion performance.

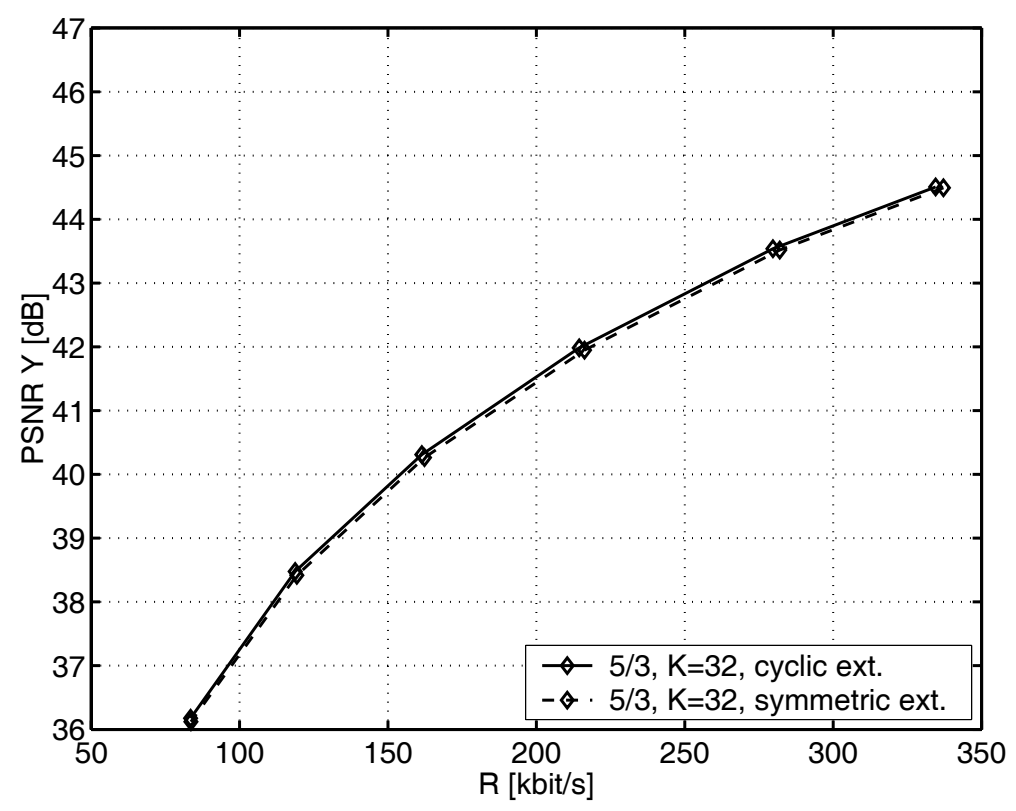

Figure 6.12: Luminance PSNR vs. total bit-rate for the QCIF sequence Container Ship at 30 fps. A dyadic decomposition is used to encode groups of $K=32$ pictures with the motioncompensated $5 / 3$ wavelet. The compression efficiency of cyclic and symmetric extension is depicted.

Fig. 6.12 shows luminance PSNR over the total bit-rate for the sequence Container Ship. We observe that both extensions show similar rate-distortion performance. We expect better efficiency for the cyclic extension as it is a multi-frame approach. But it seems that the large temporal distance is disadvantageous. As we observe a small advantage in terms of compression efficiency, also for other sequences like Foreman, we use the cyclic extension for our experiments. 


\subsection{A Mathematical Model of Motion-Compensated Subband Coding}

The experimental results show that the temporal subband coding scheme can provide superior compression efficiency when compared to the predictive coding scheme. In the following, we outline a mathematical model to study motion-compensated subband coding in more detail. With that, we derive performance bounds for motion-compensated threedimensional transform coding and compare to bounds known for motion-compensated predictive coding.

Let $\mathbf{s}_{k}=\left\{\mathbf{s}_{k}[l], l \in \Pi\right\}$ be scalar random fields over a two-dimensional orthogonal grid $\Pi$ with horizontal and vertical spacing of 1 . The vector $l=(x, y)^{T}$ denotes a particular location in the lattice $\Pi$. We interpret $\mathbf{s}_{k}$ as the $k$-th of $K$ pictures to be encoded. Further, the signal $\mathbf{s}_{k}[l]$ is thought of as samples of a space-continuous, spatially band-limited signal and we obtain a displaced version of it as follows: We shift the ideal reconstruction of the band-limited signal by the continuous-valued displacement vector $d$ and re-sample it on the original grid. With this signal model, a spatially constant displacement operation is invertible.

\subsubsection{Motion-Compensated Lifted Haar Wavelet}

With the above signal model, we revisit the motion-compensated lifted Haar wavelet in Fig. 6.1 and remove the displacement operators in the lifting steps such that we can isolate a lifted Haar wavelet without displacement operators.
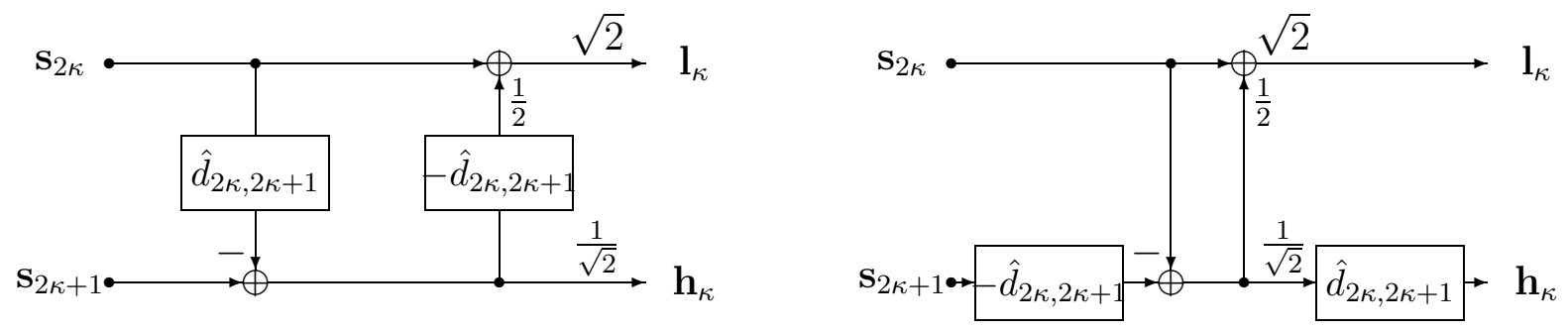

Figure 6.13: Haar transform with lifting steps that shift the signal (left). As the shift operation is invertible, an equivalent system without shifts in the lifting steps is possible (right).

Fig. 6.13 shows the equivalent Haar wavelet where the displacement operators are preand post-processing operators with respect to the original Haar transform. The schemes in Fig. 6.13 are equivalent, if the displacement operators are linear and invertible.

We continue and perform the dyadic decomposition of a GOP with the equivalent Haar wavelet. For that, the displacements of the equivalent Haar blocks have to be added. We assume that the estimated displacements between pairs of frames are additive such that, e.g., $\hat{d}_{02}+\hat{d}_{23}=\hat{d}_{03}$. As the true displacements are also additive, e.g. $d_{02}+d_{23}=d_{03}$, and differ from the estimated displacement by the displacement error, i.e. $d_{i j}=\hat{d}_{i j}+\Delta_{i j}$, we conclude that the displacement errors are also additive, e.g. $\Delta_{02}+\Delta_{23}=\Delta_{03}$, [FG03]. 


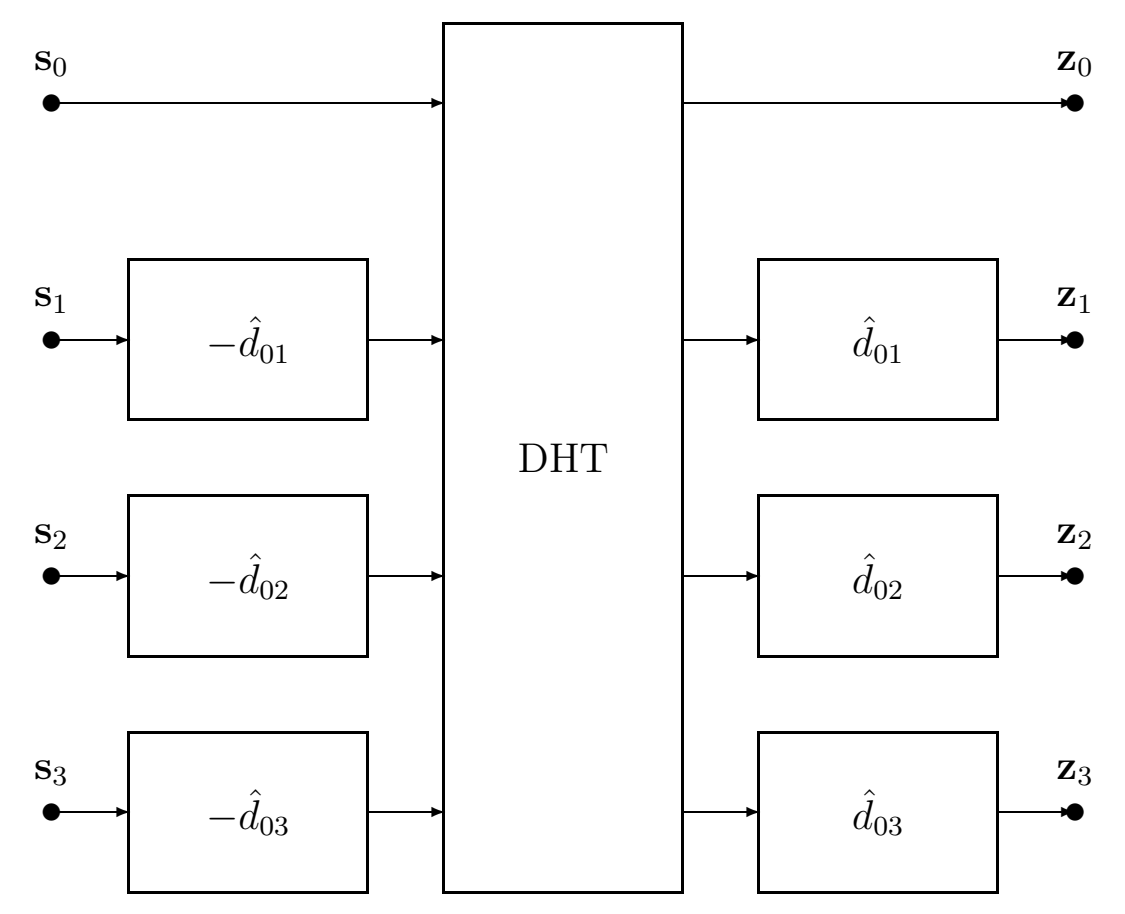

Figure 6.14: Dyadic Haar Transform (DHT) without shifts in the lifting steps for $K=4$ pictures.

Fig. 6.14 depicts a dyadic decomposition for $K=4$ pictures based on the equivalent Haar wavelet in Fig. 6.13. The dyadic Haar transform without displacements in the lifting steps is labeled by DHT. The displacements $\hat{d}_{0 k}$ are pre- and post-processing operators with respect to the original dyadic Haar decomposition DHT.

\subsubsection{Motion-Compensated Lifted 5/3 Wavelet}

We also apply the invertible displacement operator to the motion-compensated lifted $5 / 3$ wavelet in Fig. 6.2 and obtain the equivalent 5/3 wavelet in Fig. 6.15.

Due to the structure of the $5 / 3$ wavelet, we have displacements between the frames $2 \kappa$ $\& 2 \kappa+1,2 \kappa+2 \& 2 \kappa+1$, and $2 \kappa \& 2 \kappa+2$ (in the next decomposition level). Again, we assume that the estimated displacements are additive such that, e.g., $\hat{d}_{01}-\hat{d}_{21}=\hat{d}_{02}$. With this assumption, the displacement operators between the levels cancel out and several decomposition levels are possible without displacements between the levels.

The equivalent dyadic 5/3 transform has the same pre- and post-processing displacement operators as the equivalent dyadic Haar transform in Fig. 6.14 but the DHT is replaced by the original dyadic 5/3 decomposition as depicted in Fig. 6.16.

\subsubsection{Signal Model}

Now, we assume that the pictures $\mathbf{s}_{k}$ are shifted versions of a "clean" video signal $\mathbf{v}$ with the true displacements $d_{0 k}$ and distorted by independent additive white Gaussian 

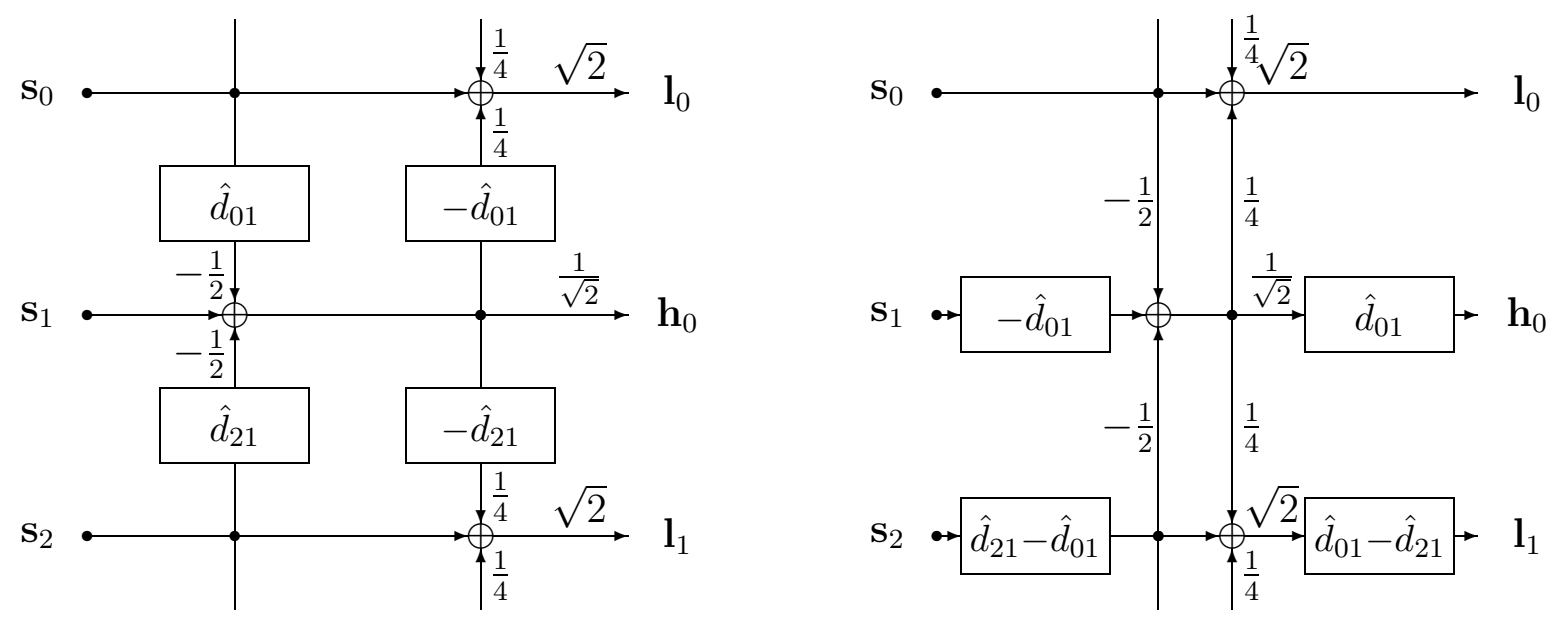

Figure 6.15: $5 / 3$ wavelet with lifting steps that shift the signal (left). As the shift operation is invertible, an equivalent system without shifts in the lifting steps is possible (right).

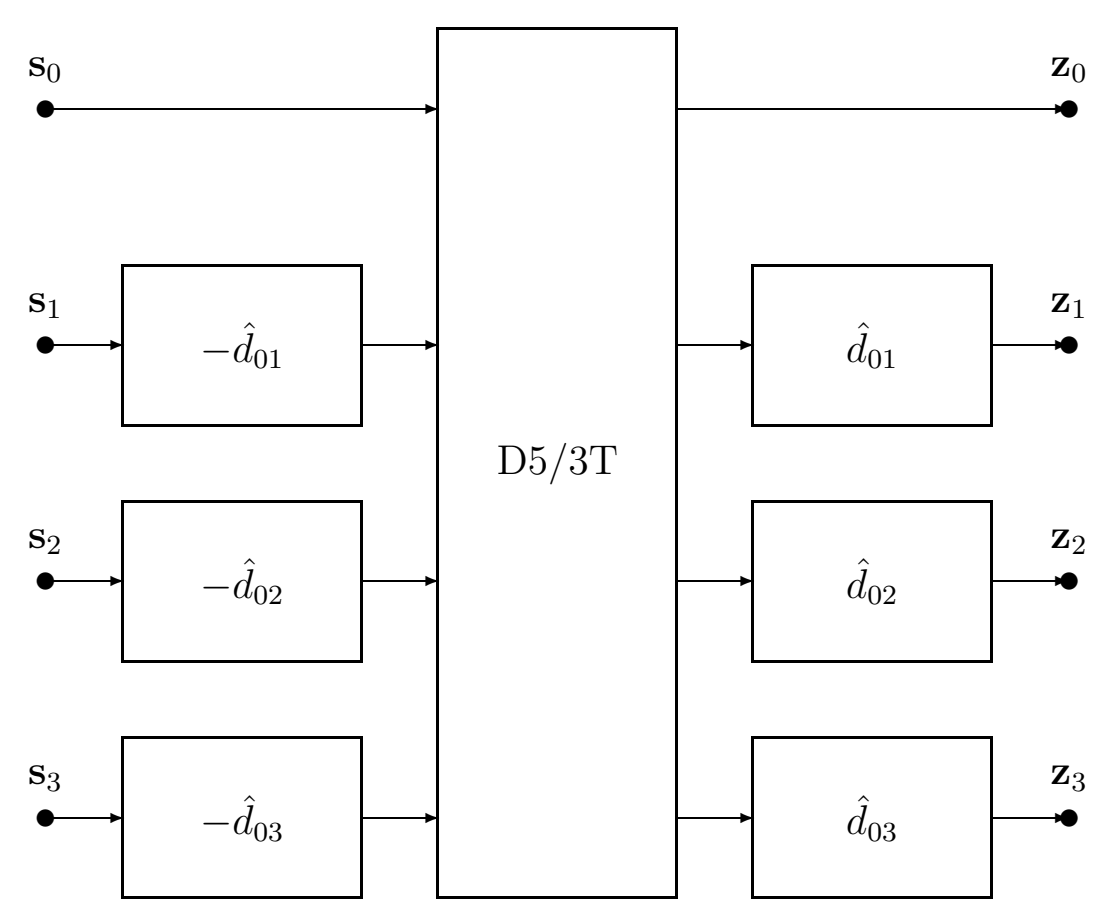

Figure 6.16: Dyadic 5/3 Transform (D5/3T) without shifts in the lifting steps for $K=4$ pictures.

noise $\mathbf{n}_{k}$. Combining this signal model with the equivalent dyadic decomposition, we can eliminate the absolute displacements and restrict ourselves to the displacement error $\Delta_{0 k}$ in the $k$-th picture. In the following, we do not consider particular displacement errors $\Delta_{0 k}$. We rather specify statistical properties and consider them as random variables $\boldsymbol{\Delta}_{k}$, statistically independent from the "clean" signal $\mathbf{v}$ and the noise $\mathbf{n}_{k}$. The noise signals $\mathbf{n}_{\mu}$ and $\mathbf{n}_{\nu}$ are also mutually statistically independent. 


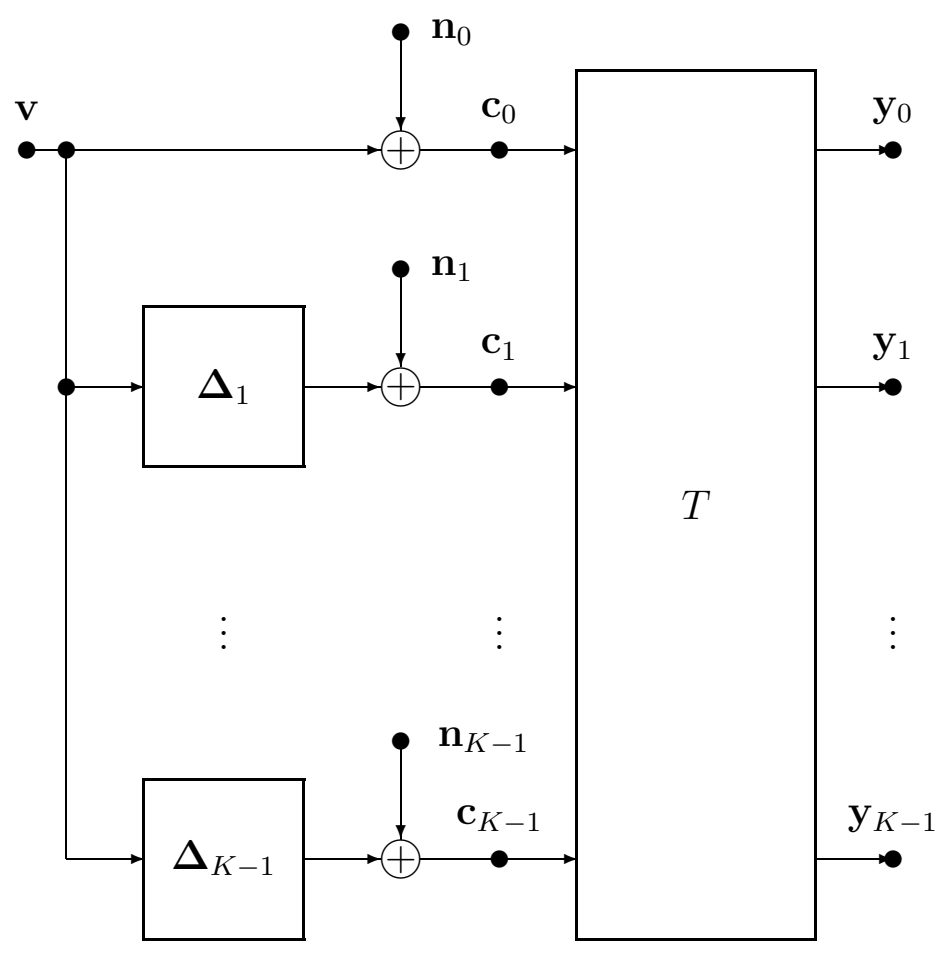

Figure 6.17: Motion compensation for a group of $K$ pictures.

Fig. 6.17 depicts the generalized model with the displacement-free and linear transform $T$ for a group of $K$ pictures. The motion-compensated pictures $\mathbf{c}_{1}, \ldots, \mathbf{c}_{K-1}$ are aligned with respect to the first picture $\mathbf{c}_{0}$. According to Fig. 6.14, the signals $\mathbf{z}_{k}$ are independently intra-frame encoded. As the absolute displacements have no influence on the performance of the intra-frame encoder, we omit them and consider only the direct output signals $\mathbf{y}_{k}$ of $T$.

Now, assume that the random fields $\mathbf{v}$ and $\mathbf{c}_{k}$ are jointly wide-sense stationary with the real-valued scalar two-dimensional power spectral densities $\Phi_{\mathbf{v v}}(\omega)$ and $\Phi_{\mathbf{c}_{\mu} \mathbf{c}_{\nu}}(\omega)$. The power spectral densities $\Phi_{\mathbf{c}_{\mu} \mathbf{c}_{\nu}}(\omega)$ are elements in the power spectral density matrix of the motion-compensated pictures $\Phi_{\mathbf{c c}}$. The power spectral density matrix of the decorrelated signal $\Phi_{\mathbf{y y}}$ is given by $\Phi_{\mathbf{c c}}$ and the transform $T$,

$$
\Phi_{\mathbf{y y}}(\omega)=T(\omega) \Phi_{\mathbf{c c}}(\omega) T^{H}(\omega),
$$

where $T^{H}$ denotes the Hermitian conjugate of $T$ and $\omega=\left(\omega_{x}, \omega_{y}\right)^{T}$ the vector-valued frequency.

We adopt the expressions for the cross spectral densities $\Phi_{\mathbf{c}_{\mu} \mathbf{c}_{\nu}}$ from [Gir00]

$$
\Phi_{\mathbf{c}_{\mu} \mathbf{c}_{\nu}}(\omega)=E\left\{e^{-j \omega^{T}\left(\boldsymbol{\Delta}_{\mu}-\boldsymbol{\Delta}_{\nu}\right)}\right\} \Phi_{\mathbf{v v}}(\omega)+\Phi_{\mathbf{n}_{\mu} \mathbf{n}_{\nu}}(\omega)
$$

and assume a normalized power spectrum $\Phi_{\mathbf{v v}}$ with $\sigma_{\mathbf{v}}^{2}=1$ that corresponds to an exponentially decaying isotropic autocorrelation function with a correlation coefficient between horizontally and vertically adjacent pixels of $\rho_{\mathbf{v}}=0.93$. 
For the $k$-th displacement error $\boldsymbol{\Delta}_{k}$, a 2-D normal distribution with variance $\sigma_{\Delta}^{2}$ and zero mean is assumed where the $x$ - and $y$-components are statistically independent. The expected value in (6.2) depends on the variance of the displacement error with respect to the reference picture $\mathbf{c}_{0}$ (absolute displacement accuracy) and the variance of the difference displacement error between pairs of non-reference pictures (relative displacement accuracy). We assume that each picture in a GOP can be the reference picture $\mathbf{c}_{0}$. That is, there is no preference among the pictures in a GOP and the variances of the absolute displacement error are the same for all $K-1$ motion-compensated pictures. Based on the dyadic decomposition with motion-compensated lifted wavelets and the assumption that there is no preference among the pictures in a GOP, we assume that absolute and relative displacement accuracy are identical. The differences of absolute displacement errors are related to the relative displacement errors as we assume in Sections 6.3.1 and 6.3.2 additive estimated displacements.

$$
\Delta_{0 j}-\Delta_{0 i}=\Delta_{i j}
$$

With that, we obtain for the variances of the absolute and relative displacement error components:

$$
\begin{aligned}
E\left\{\left(\boldsymbol{\Delta}_{0 j}-\boldsymbol{\Delta}_{0 i}\right)^{2}\right\} & =E\left\{\boldsymbol{\Delta}_{i j}^{2}\right\} \\
2 \sigma_{\boldsymbol{\Delta}}^{2}\left(1-\rho_{\boldsymbol{\Delta}}\right) & =\sigma_{\boldsymbol{\Delta}}^{2}
\end{aligned}
$$

This is only possible with correlated displacement errors such that $\rho_{\boldsymbol{\Delta}}=0.5$ [FG02b]. Finally, we abbreviate the expected value in $(6.2)$ with $P\left(\omega, \sigma_{\Delta}^{2}\right)$ which is the characteristic function of the continuous 2-D Gaussian displacement error.

$$
E\left\{e^{-j \omega^{T} \Delta_{k}}\right\}:=P\left(\omega, \sigma_{\Delta}^{2}\right)=e^{-\frac{1}{2} \omega^{T} \omega \sigma_{\Delta}^{2}}
$$

With that, we obtain for the power spectral density matrix of the motion-compensated pictures

$$
\frac{\Phi_{\mathbf{c c}}(\omega)}{\Phi_{\mathbf{v v}}(\omega)}=\left(\begin{array}{cccc}
1+\alpha(\omega) & P(\omega) & \cdots & P(\omega) \\
P(\omega) & 1+\alpha(\omega) & \cdots & P(\omega) \\
\vdots & \vdots & \ddots & \vdots \\
P(\omega) & P(\omega) & \cdots & 1+\alpha(\omega)
\end{array}\right)
$$

$\alpha=\alpha(\omega)$ is the normalized spectral density of the noise $\Phi_{\mathbf{n}_{k} \mathbf{n}_{k}}(\omega)$ with respect to the spectral density of the "clean" video signal.

$$
\alpha(\omega)=\frac{\Phi_{\mathbf{n}_{k} \mathbf{n}_{k}}(\omega)}{\Phi_{\mathbf{v v}}(\omega)} \quad \text { for } \quad k=0,1, \ldots, K-1
$$

$T$ represents the dyadic Haar transform or the dyadic 5/3 transform. In terms of decorrelation and coding gain, the $5 / 3$ wavelet performs better than the Haar wavelet as shown in Figs. 6.3 - 6.9. In the following, we are interested in theoretical performance bounds and choose the Karhunen-Loeve Transform (KLT). The normalized eigenvalues 
of the power spectral density matrix $\Phi_{\mathbf{c c}}$ are $\lambda_{1}(\omega)=1+\alpha(\omega)+(K-1) P(\omega)$ and $\lambda_{2,3, \ldots, K}(\omega)=1+\alpha(\omega)-P(\omega)$. The power spectral density matrix of the transformed signals $\Phi_{\mathbf{y y}}$ is diagonal.

$$
\frac{\Phi_{\mathbf{y y}}(\omega)}{\Phi_{\mathbf{v v}}(\omega)}=\left(\begin{array}{cccc}
1+\alpha(\omega)+(K-1) P(\omega) & 0 & \cdots & 0 \\
0 & 1+\alpha(\omega)-P(\omega) & \cdots & 0 \\
\vdots & \vdots & \ddots & \vdots \\
0 & 0 & \cdots & 1+\alpha(\omega)-P(\omega)
\end{array}\right)
$$

The first eigenvector just adds all components and scales with $1 / \sqrt{K}$. For the remaining eigenvectors, any orthonormal basis can be used that is orthogonal to the first eigenvector. That is, the KLT for our signal model is not dependent on $\omega$. Note that for this simple signal model, the Haar transform is also a KLT.

\subsubsection{Transform Coding Gain}

The rate difference [Gir00] is used to measure the improved compression efficiency for each picture $k$.

$$
\Delta R_{k}=\frac{1}{4 \pi^{2}} \int_{-\pi}^{\pi} \int_{-\pi}^{\pi} \frac{1}{2} \log _{2}\left(\frac{\Phi_{\mathbf{y}_{k} \mathbf{y}_{k}}(\omega)}{\Phi_{\mathbf{c}_{k} \mathbf{c}_{k}}(\omega)}\right) d \omega
$$

It represents the maximum bit-rate reduction (in bit per sample) possible by optimum encoding of the transformed signal $\mathbf{y}_{k}$, compared to optimum intra-frame encoding of the signal $\mathbf{c}_{k}$ for Gaussian wide-sense stationary signals for the same mean square reconstruction error. A negative $\Delta R_{k}$ corresponds to a reduced bit-rate compared to optimum intra-frame coding. The overall rate difference $\Delta R$ is the average over all pictures and is used to evaluate the efficiency of motion-compensated transform coding. Assuming the KLT, we obtain for the overall rate difference

$$
\Delta R=\frac{1}{4 \pi^{2}} \int_{-\pi}^{\pi} \int_{-\pi}^{\pi} \frac{K-1}{2 K} \log _{2}\left(1-\frac{P\left(\omega, \sigma_{\Delta}^{2}\right)}{1+\alpha(\omega)}\right)+\frac{1}{2 K} \log _{2}\left(1+(K-1) \frac{P\left(\omega, \sigma_{\Delta}^{2}\right)}{1+\alpha(\omega)}\right) d \omega .
$$

The case of a very large number of motion-compensated pictures is of special interest for the comparison to predictive video coding with motion compensation.

$$
\Delta R_{K \rightarrow \infty}=\frac{1}{4 \pi^{2}} \int_{-\pi}^{\pi} \int_{-\pi}^{\pi} \frac{1}{2} \log _{2}\left(1-\frac{P\left(\omega, \sigma_{\Delta}^{2}\right)}{1+\alpha(\omega)}\right) d \omega
$$

Note that the performance of predictive coding with motion compensation and optimum Wiener filter achieves a rate difference of

$$
\Delta R_{\mathrm{MCP}}=\frac{1}{4 \pi^{2}} \int_{-\pi}^{\pi} \int_{-\pi}^{\pi} \frac{1}{2} \log _{2}\left(1-\frac{P^{2}\left(\omega, \sigma_{\Delta}^{2}\right)}{[1+\alpha(\omega)]^{2}}\right) d \omega
$$




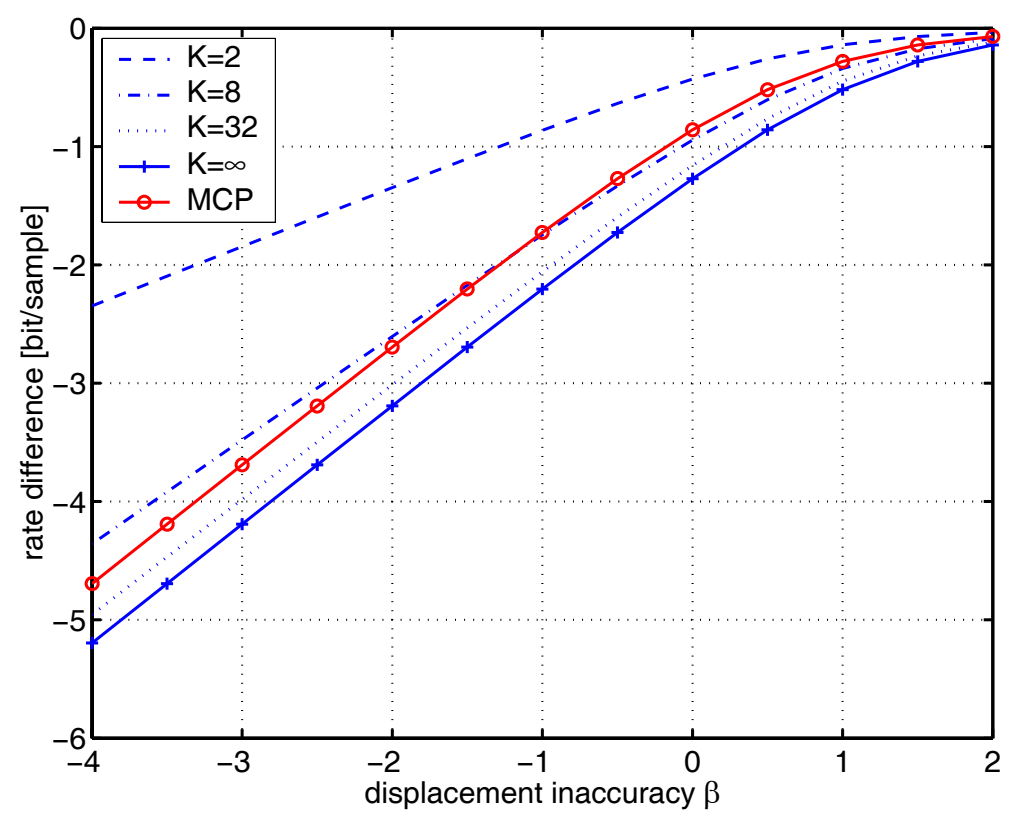

Figure 6.18: Rate difference for motion-compensated transform coding with groups of $K$ pictures over the displacement inaccuracy $\beta$. The performance of predictive coding with motion compensation and Wiener filter is labeled by MCP. The residual noise level is $-100 \mathrm{~dB}$.

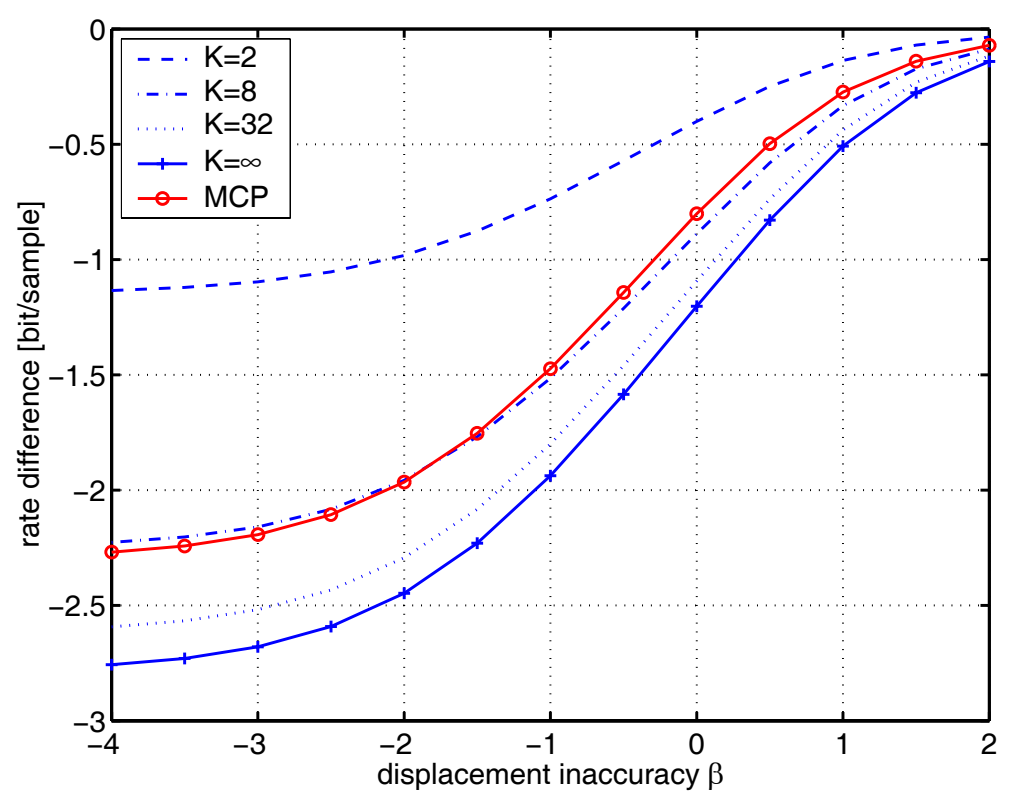

Figure 6.19: Rate difference for motion-compensated transform coding with groups of $K$ pictures over the displacement inaccuracy $\beta$. The performance of predictive coding with motion compensation and Wiener filter is labeled by MCP. The residual noise level is $-30 \mathrm{~dB}$.

We obtain this result from [Gir00], Eqn. 21 with $N=1$ and $\alpha_{0}=\alpha_{1}=\alpha$.

Figs. 6.18 and 6.19 depict the rate difference according to (6.11) and (6.13) over the displacement inaccuracy $\beta=\log _{2}\left(\sqrt{12} \sigma_{\boldsymbol{\Delta}}\right)$ for a residual noise level RNL $=10 \log _{10}\left(\sigma_{\mathbf{n}}^{2}\right)$ 
of $-100 \mathrm{~dB}$ and $-30 \mathrm{~dB}$, respectively. Note that the variance of the "clean" video signal $\mathbf{v}$ is normalized to $\sigma_{\mathbf{v}}^{2}=1$. We observe that the rate difference starts to saturate for $K=32$. This observation is consistent with the experimental results in the previous section. For a very large group of pictures and negligible residual noise, the slope of the rate difference is limited by 1 bit per sample per inaccuracy step, similar to that of predictive coding with motion compensation. Further, transform coding with motion compensation outperforms predictive coding with motion compensation by at most 0.5 bits per sample. For example, if we encode frames with statistically independent additive noise, predictive coding with motion compensation is not capable of predicting the additive noise in the current frame. In this case, prediction actually doubles the noise variance in the residual signal and predictive coding performs suboptimally. The advantage of the motion-compensated lifted $5 / 3$ wavelet over motion-compensated predictive coding is also observed in the experimental results.

\subsection{Conclusions}

This chapter discusses motion compensation for groups of $K$ pictures. We investigate experimentally and theoretically motion-compensated lifted wavelet transforms. The experiments show that the $5 / 3$ wavelet kernel outperforms both the Haar kernel and, in many cases, the reference scheme utilizing motion-compensated predictive coding. The motion-compensated lifted wavelet kernels re-use the motion vectors in the prediction step for the update step by assuming an invertible block-motion field. This assumption seems to be inadequate for sequences with inhomogeneous motion as their rate-distortion performance is weaker than expected.

The theoretical discussion is based on a signal model for $K$ motion-compensated pictures that are decorrelated by a linear transform. The dyadic decomposition of $K$ pictures with motion-compensated lifted wavelets is replaced by an equivalent coding scheme with $K$ motion-compensated pictures and a dyadic wavelet decomposition without motion compensation. That is, we remove the displacement operators in the lifting steps and generate a set of motion-compensated pictures with an additional constraint on the displacement errors. We generalize the model and employ the Karhunen-Loeve Transform to obtain theoretical performance bounds at high bit-rates for motion-compensated 3D transform coding.

The analysis of this model gives the following insights: The coding gain for a group of $K$ pictures is limited and saturates with increasing $K$. For a very large group of pictures and negligible residual noise, the slope of the rate difference is limited by 1 bit per sample per inaccuracy step. The slope of the rate difference for predictive coding with motion compensation is also limited by 1 bit per sample per inaccuracy step but this coding scheme outperforms predictive coding with motion compensation by up to 0.5 bits per sample. This is also true for very accurate motion compensation when the residual noise dominates the coding gain. 


\section{Summary}

This work discusses video coding with superimposed motion-compensated signals. We build on the theory of multihypothesis motion-compensated prediction for video coding and introduce the concept of motion compensation with complementary hypotheses. Multihypothesis motion compensation linearly combines more than one motion-compensated signal to form the superimposed motion-compensated signal. Motion-compensated signals that are used for the superposition are referred to as hypotheses. Further, a displacement error that captures the inaccuracy of motion compensation is associated with each hypothesis. As the accuracy of motion compensation is equal for all hypotheses, the displacement errors are identically distributed.

This work proposes that the multiple displacement errors are jointly distributed and, in particular, correlated. Investigations show that there is no preference among $N$ hypotheses and we conclude that all non-equal pairs of displacement errors are characterized by one correlation coefficient. As the covariance matrix of the jointly distributed displacement errors is nonnegative definite, we can determine the valid range of the displacement error correlation coefficient dependent on the number of superimposed hypotheses.

We investigate the efficiency of superimposed motion compensation as a function of the displacement error correlation coefficient. We observe that decreasing the displacement error correlation coefficient improves the efficiency of superimposed motion compensation. We conclude that motion compensation with complementary hypotheses results in maximally negatively correlated displacement error.

Motion compensation with complementary hypotheses implies two major results for the efficiency of superimposed motion-compensated prediction: First, the slope of the rate difference reaches up to 2 bits per sample per motion inaccuracy step whereas for single hypothesis motion-compensated prediction this slope is limited to 1 bit per sample per motion inaccuracy step. Here, we measure the rate difference with respect to optimum intra-frame encoding and use a high-rate approximation. Second, this slope of 2 bits per sample per inaccuracy step is already achieved for $N=2$ complementary hypotheses. If we just average the hypotheses, the performance converges at constant slope to that of a very large number of hypotheses. That is, the largest portion of the achievable gain is already accomplished with $N=2$ complementary hypotheses. If we employ the optimum Wiener filter, the coding performance improves for doubling the number of complementary hypotheses by at least 0.5 bits per sample at constant slope.

In this work, we investigate motion compensation with complementary hypotheses by integrating superimposed motion-compensated prediction into the ITU-T Rec. H.263. We linearly combine up to 4 motion-compensated blocks chosen from up to 20 previous 
reference frames to improve the performance of inter-predicted pictures. To determine the best N-hypothesis for each predicted block, we utilize an iterative algorithm that improves successively conditional optimal hypotheses. Our experiments show that superimposed prediction works efficiently for both $16 \times 16$ and $8 \times 8$ blocks. Multiple reference frames enhance the efficiency of superimposed prediction. The superposition gain and the multiframe gain do not only add up; superimposed prediction benefits from hypotheses which can be chosen from several reference frames. Superimposed prediction with two hypotheses and ten reference frames achieves coding gains up to $2.7 \mathrm{~dB}$, or equivalently, bit-rate savings up to $30 \%$ for the sequence Mobile $\&$ Calendar when compared to the one-hypothesis reference codec with one reference frame.

We explore theoretically why superimposed prediction benefits from multiframe motion compensation. We model multiframe motion compensation by forward-adaptive hypothesis switching and show that switching among $M$ hypotheses with statistically independent displacement error reduces the displacement error variance by up to a factor of $M$. For motion-compensated prediction, we obtain the following performance bounds: Doubling the number of reference pictures for single hypothesis prediction reduces the bit-rate of the residual encoder by at most 0.5 bits per sample. Whereas doubling the number of reference pictures for prediction with complementary hypotheses reduces the bit-rate of the residual encoder by at most 1 bit per sample.

This work also investigates motion compensation with complementary hypotheses for B-pictures in the emerging ITU-T Rec. H.264. We focus on reference picture selection and linearly combined motion-compensated prediction signals. We show that bidirectional prediction exploits partially the efficiency of combined prediction signals. Superimposed prediction chooses hypotheses from an arbitrary set of reference pictures and, by this, outperforms bidirectional prediction. That is, superimposed motion-compensated prediction with multiple reference frames allows a more general form of B-pictures. In addition to the generalization of the bidirectional mode, we allow that previously decoded B-pictures can be reference pictures for other B-pictures. Again, we observe that multiframe motion compensation enhances the efficiency of superimposed prediction for hybrid video coding.

Finally, we discuss superimposed motion-compensated signals for motion-compensated 3D subband coding of video. We investigate experimentally and theoretically motioncompensated lifted wavelet transforms for the temporal subband decomposition. The experiments show that the $5 / 3$ wavelet kernel outperforms both the Haar kernel and, in many cases, the reference scheme utilizing motion-compensated predictive coding. Based on the motion-compensated lifting scheme, we develop an analytical model describing motion compensation for groups of $K$ pictures.

The theoretical discussion is based on a signal model for $K$ motion-compensated pictures that are decorrelated by a linear transform. The dyadic decomposition of $K$ pictures with motion-compensated lifted wavelets is replaced by an equivalent coding scheme with $K$ motion-compensated pictures and a dyadic wavelet decomposition without motion compensation. That is, we remove the displacement operators in the lifting steps and generate a set of motion-compensated pictures with an additional constraint on the displacement errors. We generalize the model and employ the Karhunen-Loeve Transform to obtain 
theoretical performance bounds at high bit-rates for motion-compensated 3D transform coding.

The analysis of this model gives the following insights: The coding gain for a group of $K$ pictures is limited and starts to saturate for $K=32$ pictures. For a very large group of pictures and negligible residual noise, the slope of the rate difference is limited by 1 bit per sample per inaccuracy step. The slope of the rate difference for motion-compensated prediction is also limited by 1 bit per sample per inaccuracy step but this coding scheme outperforms motion-compensated prediction by at most 0.5 bits per sample. This is also true for very accurate motion compensation when the residual noise dominates the coding gain.

In summary, we characterize the relation between motion-compensated signals and, depending on this relation, investigate their efficiency for video compression.

\section{Zusammenfassung ${ }^{1}$}

Diese Arbeit erörtert Videocodierung mit überlagerten bewegungskompensierten Signalen. Es wird die Theorie der bewegungskompensierten Multihypothesen-Prädiktion für die Videocodierung erweitert und das Konzept der Prädiktion mit komplementären bewegungskompensierten Signalen eingeführt. Bewegungskompensierte MultihypothesenPrädiktion überlagert linear mehr als ein bewegungskompensiertes Signal um das Prädiktionssignal zu bestimmen. Bewegungskompensierte Signale, die für die Überlagerung herangezogen werden, nennt man auch Hypothesen. Für das Modell wird jede Hypothese mit einem Versatzfehler assoziiert, der die Ungenauigkeit der Bewegungskompensation erfasst. Es wird angenommen, dass individuelle Versatzfehler identische Wahrscheinlichkeitsverteilungen aufweisen, da keine der Hypothesen sich auszeichnet.

Es wird vorgeschlagen, dass die Versatzfehler korreliert und durch eine Verbundwahrscheinlichkeit gekennzeichnet sind. Da keine der $N$ Hypothesen sich auszeichnet, sind alle Paare mit nicht-identischen Versatzfehlern durch einen gemeinsamen Korrelationskoeffizient charakterisiert. Die Kovarianzmatrix der verbundverteilten Versatzfehler ist nicht-negativ definit und bestimmt den zulässigen Bereich des Versatzfehler-Korrelationskoeffizienten in Abhängigkeit der Anzahl der zu überlagernden Hypothesen.

Es wird die Effizienz der bewegungskompensierten Multihypothesen-Prädiktion in Abhängigkeit des Versatzfehler-Korrelationskoeffizienten untersucht. Es zeigt sich, dass eine Verringerung dieses Koeffizienten die Effizienz der bewegungskompensierten Multihypothesen-Prädiktion verbessert. Prädiktion mit komplementären bewegungskompensierten Signalen ist durch maximal negativ-korrelierte Versatzfehler gekennzeichnet.

Prädiktion mit komplementären Hypothesen hat für die Effizienz der bewegungskompensierten Multihypothesen-Prädiktion zwei wichtige Ergebnisse zur Folge: Erstens, die Steigung der Ratendifferenz erreicht bis zu 2 bits pro Abtastwert und Bewegungsungenauigkeitsschritt. Diese Steigung ist bei der bewegungskompensierten Prädiktion mit einer

\footnotetext{
${ }^{1}$ This translation of the summary is a requirement according to the "Prüfungsordnung für die Technischen Fakultät der Friedrich-Alexander-Universität Erlangen-Nürnberg".
} 
Hypothese auf 1 bit pro Abtastwert und Bewegungsungenauigkeitsschritt beschränkt. Die Ratendifferenz wird hier bezüglich der optimalen Intra-Bild Codierung unter Verwendung einer Hochraten-Approximation gemessen. Zweitens, die Steigung von 2 bits pro Abtastwert und Ungenauigkeitsschritt wird bereits für $N=2$ komplementäre Hypothesen erreicht. Werden mehr als zwei komplementäre Hypothesen gemittelt, dann konvergiert die Ratendifferenz bei konstanter Steigung zu einem Wert, der mit der Mittelung sehr vieler Hypothesen erreichen werden kann. Aus diesem Grund wird bereits mit $N=2$ komplementären Hypothesen der größte Anteil des möglichen Gewinns erzielt. Werden jedoch mehr als zwei komplementäre Hypothesen mit dem optimalen Wiener Filter überlagert, dann verbessert sich die Ratendifferenz bei gleicher Steigung um mindestens 0.5 bits pro Abtastwert bei Verdoppelung der Hypothesenzahl.

Die vorliegenden Arbeit untersucht die Prädiktion mit komplementären Hypothesen indem die ITU-T Rec. H.263 um überlagerte bewegungskompensierte Prädiktion erweitert wird. Zur Verbesserung der Inter-Bilder werden bis zu 4 bewegungskompensierte Blöcke überlagert, die von bis zu 20 vorangehenden Referenzbildern ausgewählt werden. Ein iterativer Algorithmus, der sukzessiv bedingt-optimale Hypothesen verbessert, wird verwendet um für jeden zu prädizierenden Block die beste $N$-Hypothese zu bestimmen. Die Experimente zeigen, dass überlagerte Prädiktion für sowohl $16 \times 16$ als auch $8 \times 8$ Blöcke von Vorteil ist. Mehrere Referenzbilder verbessern die Codiereffizienz der überlagerten Prädiktion. Die Gewinne durch überlagerte Prädiktion und mehrere Referenzbilder sind nicht nur additiv; überlagerte Prädiktion profitiert von Hypothesen, die von verschiedenen Referenzbildern ausgewählt werden können. Im Vergleich zu einem Codec mit bewegungskompensierter Prädiktion und einem Referenzbild erzielt überlagerte Prädiktion mit zwei Hypothesen und zehn Referenzbildern Qualitätsverbesserungen bis zu $2.7 \mathrm{~dB}$, oder äquivalent, Bitratenersparnisse bis zu $30 \%$ für die Bildsequenz Mobile $\&$ Calendar.

Die Beobachtung, dass überlagerte Prädiktion von der Bewegungskompensation mit mehreren Referenzbildern profitiert, wird eingehender untersucht. Dabei wird die Bewegungskompensation mit mehreren Referenzbildern durch die vorwärts-adaptive Auswahl von bewegungskompensierten Signalen modelliert. Es kann gezeigt werden, dass die Auswahl unter $M$ Hypothesen mit statistisch unabhängigen Versatzfehlern die Versatzfehlervarianz höchstens um den Faktor $M$ reduziert. Folgende Ergebnisse werden für die Prädiktion mit komplementären Hypothesen erzielt: Eine Verdoppelung der Anzahl der Referenzbilder bei der bewegungskompensierten Prädiktion reduziert die Bitrate des Differenzbild-Encoders höchstens um 0.5 bits pro Abtastwert. Aber eine Verdoppelung der Anzahl der Referenzbilder bei der Prädiktion mit komplementären Hypothesen reduziert diese Bitrate bis zu 1 bit pro Abtastwert.

Diese Arbeit untersucht auch bei B-Bildern die Prädiktion mit komplementären Hypothesen und verbessert diese für die neue ITU-T Rec. H.264. Dazu wird die Referenzbildauswahl für linear überlagerte bewegungskompensierte Signale diskutiert. Es wird gezeigt, dass die bi-direktionale Prädiktion die potentielle Effizienz der überlagertern Prädiktion nur teilweise nutzt. Überlagerte Prädiktion wählt aus einer beliebigen Referenzbildmenge die effizientesten Hypothesen aus und ist dadurch bi-direktionaler Prädiktion überlegen. Dies bedeutet, dass überlagerte Prädiktion mit mehreren Referenzbildern eine allgemeine- 
re Form von B-Bildern zulässt. Desweiteren können vorher decodierte B-Bilder Referenzbilder für andere verallgemeinerte B-Bilder sein. Die Ergebnisse für B-Bilder zeigen, dass die Bewegungskompensation mit mehreren Referenzbildern die Effizienz der überlagerten Prädiktion verbessert.

Schließlich werden linear überlagerte bewegungskompensierte Signale für die bewegungskompensierte 3D Teilbandcodierung von Videosignalen diskutiert. Für die zeitliche Teilbandcodierung werden experimentell und theoretisch bewegungskompensierte Wavelet-Transformationen untersucht. Die Experimente zeigen, dass das 5/3 Wavelet dem Haar Wavelet, und in vielen Fällen, dem Referenzverfahren mit bewegungskompensierter prädiktiver Codierung überlegen ist. Basierend auf den untersuchten Wavelet-Verfahren wird ein analytisches Modell zur Beschreibung der Bewegungskompensation für Gruppen von $K$ Bildern entwickelt.

Die theoretische Diskussion basiert auf einem Signalmodell für $K$ bewegungskompensierte Bilder, die durch eine lineare Transformation dekorreliert werden. Dazu werden dyadische Zerlegungen von $K$ Bildern mit bewegungskompensierten Wavelets durch äquivalente Codierverfahren mit $K$ bewegungskompensierten Bildern und dyadischen Wavelet-Zerlegungen ohne Bewegungskompensation ersetzt. Dabei werden die Versatzoperatoren in den Hebestufen der Wavelets entfernt und eine Gruppe von bewegungskompensierten Bildern erzeugt, die durch eine zusätzliche Bedingung für die Versatzfehler charakterisiert werden. Das Modell wird durch Anwendung der Karhunen-Loeve Transformation verallgemeinert und erlaubt eine Diskussion theoretischer Schranken für die bewegungskompensierte 3D Transformationscodierung bei hohen Bitraten.

Die Analyse dieses Modells führt zu folgenden Einsichten: Der Codiergewinn für eine Gruppe von $K$ Bildern ist beschränkt und neigt ab $K=32$ Bildern zur Sättigung. Für eine sehr große Anzahl von Bildern und vernachlässigbarem Rauschen ist die Steigung der Ratendifferenz auf 1 bit pro Abtastwert und Ungenauigkeitsschritt beschränkt. Die Steigung der Ratendifferenz für bewegungskompensierte Prädiktion ist auch auf 1 bit pro Abtastwert und Ungenauigkeitsschritt beschränkt, jedoch übertrifft dieses Codierverfahren die bewegungskompensierte Prädiktion um höchstens 0.5 bits pro Abtastwert. Dies trifft auch für sehr genaue Bewegungskompensation zu, wenn der Codiergewinn durch das additive Rauschen bestimmmt wird.

Zusammenfassend lässt sich festhalten, dass die Beziehung zwischen bewegungskompensierten Signalen charakterisiert, und in Abhängigkeit dieser Beziehung, ihre Effizienz für die Videocompression untersucht wird. 


\section{A Mathematical Results}

\section{A.1 Singularities of the Displacement Error Covari- ance Matrix}

The prediction scheme shows no preferences among the individual hypotheses. This is reflected in the symmetry of the displacement error covariance matrix. The variance of the displacement error for each hypothesis is $\sigma_{\boldsymbol{\Delta}}^{2}$ and the correlation coefficient between any hypothesis pair is $\rho_{\Delta}$. With that, the covariance matrix of the displacement error vector reads

$$
C=\sigma_{\Delta}^{2}\left(\begin{array}{cccc}
1 & \rho_{\Delta} & \cdots & \rho_{\Delta} \\
\rho_{\Delta} & 1 & \cdots & \rho_{\Delta} \\
\vdots & \vdots & \ddots & \vdots \\
\rho_{\Delta} & \rho_{\Delta} & \cdots & 1
\end{array}\right)
$$

In order to determine the singularities of the matrix, we decompose the covariance matrix into the identity matrix $I$ and the matrix $11^{T}$ with each element equal to one.

$$
C=\sigma_{\Delta}^{2} \rho_{\Delta} \mathbf{1 1 ^ { T }}-\sigma_{\boldsymbol{\Delta}}^{2}\left(\rho_{\boldsymbol{\Delta}}-1\right) I
$$

The covariance matrix is singular if its determinant is zero

$$
\operatorname{det}\left(11^{T}-\frac{\rho_{\boldsymbol{\Delta}}-1}{\rho_{\boldsymbol{\Delta}}} I\right)=0 .
$$

The eigenvalues of the matrix $\mathbf{1 1}^{T}$ are $\lambda=\{N, 0\}$ which can also be obtained by solving $\operatorname{det}\left(\mathbf{1 1}^{T}-\lambda I\right)=0$. We obtain two singularities for the covariance matrix:

$$
\rho_{\Delta}=\frac{1}{1-N} \quad \text { and } \quad \rho_{\Delta}=1
$$

\section{A.2 A Class of Matrices and their Eigenvalues}

We consider the following class of normalized $N \times N$ matrices with the parameter $a \in \mathcal{R}$ :

$$
C=\left(\begin{array}{cccc}
1 & a & \cdots & a \\
a & 1 & \cdots & a \\
\vdots & \vdots & \ddots & \vdots \\
a & a & \cdots & 1
\end{array}\right)
$$


In order to determine the eigenvalues of $C$, we decompose the matrix into the identity matrix $I$ and the matrix $\mathbf{1 1 ^ { T }}$ with each element equal to one.

$$
C=a 11^{T}+(1-a) I
$$

The eigenvalues $\lambda_{i}$ of $C$ solve $\operatorname{det}\left(C-\lambda_{i} I\right)=0$ and with (A.6), we can write for $a \neq 0$

$$
\operatorname{det}\left(1 \mathbf{1}^{T}-\frac{\lambda_{i}-1+a}{a} I\right)=0 .
$$

The eigenvalues of the matrix $\mathbf{1 1 ^ { T }}$ are $\lambda_{1}=N, 1$-fold, and $\lambda_{2}=0,(N-1)$-fold. Both can be obtained by solving $\operatorname{det}\left(\mathbf{1 1}^{T}-\lambda_{i} I\right)=0$. With $a \neq 0$, we have the following eigenvalues for $C$ :

$$
\begin{array}{lll}
\lambda_{1}=1+(N-1) a & & 1 \text {-fold } \\
\lambda_{2}=1-a & & (N-1) \text {-fold }
\end{array}
$$

If $a=0$, the $N$-fold eigenvalue of $C$ is 1 .

\section{A.3 Inverse of the Power Spectral Density Matrix}

The optimum Wiener filter requires the inverse of the hypothesis power spectral density matrix $\Phi_{\mathbf{c c}}(\omega)$. After normalization, the matrix $H_{1}$ of the form

$$
H_{1}=\left(\begin{array}{cccc}
c & 1 & \cdots & 1 \\
1 & c & \cdots & 1 \\
\vdots & \vdots & \ddots & \vdots \\
1 & 1 & \cdots & c
\end{array}\right)
$$

is inverted. In the following, we prefer a representation of $H_{1}$ with the vector $\mathbf{1}=$ $(1,1, \ldots, 1)^{T}$ and the identity matrix $I$

$$
H_{1}=11^{T}+(c-1) I
$$

Due to the symmetry of $H_{1}$, its inverse shows the same structure

$$
H_{1}^{-1}=k\left[11^{T}+(d-1) I\right],
$$

where $k$ and $d$ are scalar constants. As the inverse of the non-singular matrix $(c \neq 1$, $c \neq 1-N)$ is unique, we obtain

$$
\begin{aligned}
k & =\frac{1}{(c-1)(1-c-N)}, \\
d & =2-c-N
\end{aligned}
$$

and for the inverse

$$
H_{1}^{-1}=\frac{\mathbf{1 1}^{T}+(1-c-N) I}{(c-1)(1-c-N)} .
$$


In the case that the noise variance for each hypothesis is not identical, a normalized matrix

$$
H_{2}=\left(\begin{array}{cccc}
c_{1} & a_{1} a_{2} & \cdots & a_{1} a_{N} \\
a_{2} a_{1} & c_{2} & \cdots & a_{2} a_{N} \\
\vdots & \vdots & \ddots & \vdots \\
a_{N} a_{1} & a_{N} a_{2} & \cdots & c_{N}
\end{array}\right)
$$

is inverted. A representation of $H_{2}$ with the vector $a=\left(a_{1}, a_{2}, \ldots, a_{N}\right)^{T}$ and the diagonal matrix $\operatorname{diag}(\cdot)$ reads

$$
H_{2}=a a^{T}+\operatorname{diag}\left(c_{i}-a_{i}^{2}\right)
$$

The inverse shows the same symmetry as $H_{2}$ :

$$
H_{2}^{-1}=k\left[b b^{T}+\operatorname{diag}\left(d_{i}-b_{i}^{2}\right)\right],
$$

where $k$ is a scalar constant and $b$ and $d$ are vectors of size $N$. As the inverse of a non-singular matrix is unique, the parameters of $H_{2}^{-1}$ can be calculated for $a_{i}^{2} \neq c_{i}$, $i=1,2, \ldots, N$, and $k \neq 0$ with

$$
\begin{aligned}
k & =\frac{1}{\sum_{j=1}^{N} \frac{a_{j}^{2}}{a_{j}^{2}-c_{j}}-1}, \\
b_{i} & =\frac{a_{i}}{a_{i}^{2}-c_{i}}, \\
d_{i} & =\frac{1}{a_{i}^{2}-c_{i}}\left[\frac{a_{i}^{2}}{a_{i}^{2}-c_{i}}-\frac{1}{k}\right] .
\end{aligned}
$$

A simplified expression for the inverse reads

$$
H_{2}^{-1}=\frac{b b^{T}}{\sum_{j=1}^{N} \frac{a_{j}^{2}}{a_{j}^{2}-c_{j}}-1}-\operatorname{diag}\left(\frac{1}{a_{i}^{2}-c_{i}}\right) \quad \text { with } \quad b_{i}=\frac{a_{i}}{a_{i}^{2}-c_{i}} .
$$

This solution for $H_{2}^{-1}$ is only valid for $a_{i}^{2} \neq c_{i}$ and $k \neq 0$.

The special case $a=\mathbf{1}$ is also of interest for the hypothesis power spectrum matrix. $\mathrm{H}_{3}$ reads

$$
H_{3}=\left(\begin{array}{cccc}
c_{1} & 1 & \cdots & 1 \\
1 & c_{2} & \cdots & 1 \\
\vdots & \vdots & \ddots & \vdots \\
1 & 1 & \cdots & c_{N}
\end{array}\right)
$$

and its inverse can be calculated by

$$
H_{3}^{-1}=\frac{b b^{T}}{\sum_{j=1}^{N} \frac{1}{1-c_{j}}-1}-\operatorname{diag}\left(\frac{1}{1-c_{i}}\right) \quad \text { with } \quad b_{i}=\frac{1}{1-c_{i}}
$$

This solution holds only for $c_{i} \neq 1, i=1,2, \ldots, N$, and $\sum_{j=1}^{N} \frac{1}{1-c_{j}} \neq 1$. 


\section{A.4 Power Spectral Density of a Frame}

The 2D continuous-space Fourier transform $\mathcal{F}\{\cdot\}$ of the 2D continuous signal $v(x, y)$ in Cartesian coordinates with $x, y \in \mathcal{R}$ is defined by

$$
V\left(\omega_{x}, \omega_{y}\right)=\int_{\mathcal{R}^{2}} v(x, y) e^{-j\left(\omega_{x} x+\omega_{y} y\right)} d x d y .
$$

The 2D continuous-space Fourier transform of the 2D continuous signal $\stackrel{\circ}{v}(r, \theta)$ in cylindrical coordinates is given by

$$
\stackrel{\circ}{V}\left(\omega_{r}, \omega_{\theta}\right)=\int_{0}^{2 \pi} \int_{0}^{\infty} \stackrel{\circ}{v}(r, \theta) e^{-j r \omega_{r} \cos \left(\theta-\omega_{\theta}\right)} r d r d \theta
$$

where

$$
\begin{array}{ll}
x=r \cos \theta, & \omega_{x}=\omega_{r} \cos \omega_{\theta}, \\
y=r \sin \theta, & \omega_{y}=\omega_{r} \sin \omega_{\theta} .
\end{array}
$$

The Fourier transform of the isotropic, exponentially decaying, space-continuous function

$$
\stackrel{\circ}{v}(r, \theta)=e^{-\omega_{0} r} \quad \forall \theta
$$

with $\omega_{0}>0$ is also isotropic.

$$
\begin{aligned}
\stackrel{\circ}{V}\left(\omega_{r}, \omega_{\theta}\right) & =\int_{0}^{2 \pi} \int_{0}^{\infty} e^{-\left[\omega_{0}+j \omega_{r} \cos (\theta)\right] r} r d r d \theta \\
& =\int_{0}^{2 \pi} \frac{1}{\left[\omega_{0}+j \omega_{r} \cos (\theta)\right]^{2}} d \theta \\
& =\frac{1}{\omega_{0}^{2}} \int_{0}^{2 \pi} \frac{1}{[1+j \Omega \cos (\theta)]^{2}} d \theta \quad \text { with } \Omega=\frac{\omega_{r}}{\omega_{0}} \\
& =\frac{2 j}{\omega_{0}^{2}} \int_{1+j \Omega}^{1-j \Omega} \frac{1}{x^{2} \sqrt{x^{2}-2 x+1+\Omega^{2}}} d x \quad \text { with } \quad x=1+j \Omega \cos (\theta) \\
& =\frac{2 \pi}{\omega_{0}^{2}}\left(1+\Omega^{2}\right)^{-\frac{3}{2}} \\
& =\frac{2 \pi}{\omega_{0}^{2}}\left(1+\frac{\omega_{r}^{2}}{\omega_{0}^{2}}\right)^{-\frac{3}{2}}
\end{aligned}
$$

The integral in (A.32) is solved in [BS91b, BS91a]. In Cartesian coordinates, the Fourier transform of the function $v(x, y)=e^{-\omega_{0} \sqrt{x^{2}+y^{2}}}$ reads

$$
V\left(\omega_{x}, \omega_{y}\right)=\frac{2 \pi}{\omega_{0}^{2}}\left(1+\frac{\omega_{x}^{2}+\omega_{y}^{2}}{\omega_{0}^{2}}\right)^{-\frac{3}{2}} .
$$




\section{Glossary}

\section{Acronyms}

BL Baseline

CABAC Context-Based Adaptive Binary Arithmetic Coding

CIF Common Intermediate Format

DCT Discrete Cosine Transform

DHT Dyadic Haar Transform

D5/3T Dyadic 5/3 Transform

ECVQ Entropy Constrained Vector Quantization

GOP Group of Pictures

HP Half-Pel

INTER4V Inter-prediction mode with four $8 \times 8$ blocks

INTER2H Inter-prediction mode with two hypotheses

INTER4H Inter-prediction mode with four hypotheses

INTER4VMH Inter-prediction mode with four $8 \times 8$ superimposed blocks

IP Integer-Pel

ITU International Telecommunication Union

ITU-T ITU Telecommunication Standardization Sector

KLT Karhunen-Loeve Transform

MCP Motion-Compensated Prediction

MHP Superimposed Prediction

MPEG Moving Picture Experts Group

OBMC Overlapped Block Motion Compensation

PDF Probability Density Function

PSNR Peak Signal to Noise Ratio

QCIF Quarter Common Intermediate Format 
RNL Residual Noise Level

TML-9 H.26L Test Model Software Version 9

UVLC Universal Variable Length Coding

VBS Variable Block Size

VCEG Video Coding Experts Group

VQ Vector Quantization

\section{Probability Theory}

$\begin{array}{ll}\mathcal{R} & \text { Set of real numbers } \\ \Pi & \text { Two-dimensional orthogonal unit grid } \\ \mathbf{a} & \text { Random variable, process, or field } \\ \operatorname{Pr}\{\mathbf{a} \leq a\} & \text { Probability of the event }\{\mathbf{a} \leq a\} \\ R_{\mathbf{a}}(a) & \text { Reliability function of } \mathbf{a} \\ p_{\mathbf{a}}(a) & \text { PDF of } \mathbf{a} \\ \sigma_{\mathbf{a}}^{2} & \text { Variance of } \mathbf{a} \\ \rho_{\mathbf{a}} & \text { Correlation coefficient associated with } \mathbf{a} \\ \phi_{\mathbf{a b}}(\cdot) & \text { Correlation function between } \mathbf{a} \text { and } \mathbf{b} \\ \Phi_{\mathbf{a b}}(\cdot) & \text { Cross spectral density of } \mathbf{a} \text { and } \mathbf{b} \\ E\{\cdot\} & \text { Expectation operator }\end{array}$

\section{Matrix Algebra}

1 Column vector with all entries equal to one

$\|\cdot\|_{2}^{2} \quad$ Square norm of a vector

$|\cdot| \quad$ Length of a code word vector

$\operatorname{det}(\cdot) \quad$ Determinant of a matrix

$\operatorname{diag}(\cdot) \quad$ Diagonal matrix

I Identity matrix

* Complex conjugate operator

${ }^{H} \quad$ Hermitian conjugate operator

.$^{T} \quad$ Transpose operator 


\section{Transforms}
$\mathcal{F}_{*}\{\cdot\}$
2D band-limited discrete-space Fourier transform
$\mathcal{F}\{\cdot\}$
2D continuous-space Fourier transform 


\section{Bibliography}

[ATT90] T. Akiyama, T. Takahashi, and K. Takahashi. Adaptive three-dimensional transform coding for moving pictures. In Proceedings of the Picture Coding Symposium, pages 8.2-1-8.2-2, Cambridge, MA, March 1990.

[BBFPP01] V. Bottreau, M. Benetiere, B. Felts, and B. Pesquet-Popescu. A fully scalable 3D subband video codec. In Proceedings of the IEEE International Conference on Image Processing, pages 1017-1020, Thessaloniki, Greece, October 2001.

[Ber71] T. Berger. Rate Distortion Theory: A Mathematical Basis for Data Compression. Prentice-Hall, Englewood Cliffs, NJ, 1971.

[Bes86] J. Besag. On the statistical analysis of dirty pictures. J. Roy. Statist. Soc. $B, 48(3): 259-302,1986$.

[BG96] M. Budagavi and J.D. Gibson. Multiframe block motion compensated video coding for wireless channels. In Thirtieth Asilomar Conference on Signals, Systems and Computers, volume 2, pages 953-957, November 1996.

[BG97] M. Budagavi and J.D. Gibson. Error propagation in motion compensated video over wireless channels. In Proceedings of the IEEE International Conference on Image Processing, pages 89-92, Santa Barbara, CA, October 1997.

[BG98] M. Budagavi and J.D. Gibson. Random lag selection in multiframe motion compensation. In Proceedings of the International Symposium on Information Theory, page 410, Cambridge, MA, August 1998.

[BG01] M. Budagavi and J.D. Gibson. Multiframe video coding for improved performance over wireless channels. IEEE Transactions on Image Processing, 10(2):252-265, February 2001.

[BLB92] F. Bosveld, R.L. Lagendijk, and J. Biemond. Hierarchical video coding using a spatio-temporal subband decomposition. In Proceedings of the IEEE International Conference on Acoustics, Speech and Signal Processing, volume 3, pages 221-224, San Francisco, CA, March 1992. 
[BS91a] I.N. Bronstein and K.A Semendjajew. Ergänzende Kapitel zum Taschenbuch der Mathematik. Verlag Harri Deutsch, Frankfurt/Main, 1991.

[BS91b] I.N. Bronstein and K.A. Semendjajew. Taschenbuch der Mathematik. Verlag Harri Deutsch, Frankfurt/Main, 1991.

[Cas96] R. Castagno. A method for motion adaptive frame rate up-conversion. IEEE Transactions on Circuits and Systems for Video Technology, 6(5):436-446, October 1996.

[CAW96] M.C. Chen and Jr. A.N. Willson. Rate-distortion optimal motion estimation algorithm for video coding. In Proceedings of the IEEE International Conference on Acoustics, Speech and Signal Processing, pages 2096-2099, Atlanta, GA, May 1996.

[CAW98] M.C. Chen and Jr. A.N. Willson. Rate-distortion optimal motion estimation algorithms for motion-compensated transform video coding. IEEE Transactions on Circuits and Systems for Video Technology, 8(2):147-158, April 1998.

[CBN98] R.L. Claypoole, R.G. Baraniuk, and R.D. Nowak. Adaptive wavelet transforms via lifting. In Proceedings of the IEEE International Conference on Acoustics, Speech and Signal Processing, pages 1513-1516, Seattle, Washington, May 1998.

[CC96] C.-H. Chou and C.-W. Chen. A perceptually optimized 3-D subband codec for video communication over wireless channels. IEEE Transactions on Circuits and Systems for Video Technology, 6(2):143-156, April 1996.

[CDSB97] R.L. Claypoole, G. Davis, W. Sweldens, and R.G. Baraniuk. Nonlinear wavelet transforms for image coding. In Proceedings of the 31st IEEE Asilomar Conference on Signals, Systems, and Computers, volume 1, pages 662667, Pacific Grove, CA, November 1997.

[CDSY98] R. Calderbank, I. Daubechies, W. Sweldens, and B.-L. Yeo. Wavelet transforms that map integers to integers. Appl. Comput. Harmon. Anal., 5(3):332369, July 1998.

[CEGK98] G. Côté, B. Erol, M. Gallant, and F. Kossentini. H.263+: Video coding at low bit rates. IEEE Transactions on Circuits and Systems for Video Technology, 8(7):849-866, November 1998.

[CKS96] W.C. Chung, F. Kossentini, and M.J.T. Smith. An efficient motion estimation technique based on a rate-distortion criterion. In Proceedings of the IEEE International Conference on Acoustics, Speech and Signal Processing, pages 1926-1929, Atlanta, GA, May 1996. 
[CLG89] P.A. Chou, T. Lookabaugh, and R.M. Gray. Entropy-constrained vector quantization. IEEE Transactions on Acoustics, Speech and Signal Processing, 37:31-42, January 1989.

[CRT90] C. Cafforio, F. Rocca, and S. Tubaro. Motion compensated image interpolation. IEEE Transactions on Communications, 38(2):215-222, February 1990.

[CT91] T.M. Cover and J.A. Thomas. Elements of Information Theory. John Wiley \& Sons, New York, 1991.

[CV96a] L. Cuvelier and L. Vandendorpe. Coding of interlaced or progressive video sources: A theoretical analysis. In Proceedings of the IEEE International Conference on Image Processing, pages 949-952, Lausanne, Switzerland, September 1996.

[CV96b] L. Cuvelier and L. Vandendorpe. Motion estimation and compensation for CIF/SIF video sequences. In Proceedings of the IEEE Digital Signal Processing Workshop, pages 21-24, Loen, Norway, September 1996.

[CW99] S.-J. Choi and J.W. Woods. Motion-compensated 3-d subband coding of video. IEEE Transactions on Image Processing, 8(2):155-167, February 1999.

[CW00] L.A. Da Silva Cruz and J.W. Woods. Adaptive motion vector vector quantization for video coding. In Proceedings of the IEEE International Conference on Image Processing, volume 2, pages 867-870, Vancouver, Canada, September 2000 .

[CW02] P. Chen and J.W. Woods. Video coding for digital cinema. In Proceedings of the IEEE International Conference on Image Processing, volume 1, pages 749-752, Rochester, NY, September 2002.

[DS84] E. Dubois and S. Sabri. Noise reduction in image sequences using motioncompensated temporal filtering. IEEE Transactions on Communications, COM-32(7):826-831, July 1984.

[DS98] I. Daubechies and W. Sweldens. Factoring wavelet transforms into lifting steps. J. Fourier Anal. Appl., 4:247-269, 1998.

[Eri85] S. Ericsson. Fixed and adaptive predictors for hybrid predictive/transform coding. IEEE Transactions on Communications, 33(12):1291-1302, December 1985 .

[Eve63] H. Everett III. Generalized lagrange multiplier method for solving problems of optimum allocation of resources. Operations Research, 11:399-417, 1963. 
[FAM97] T. Fukuhara, K. Asai, and T. Murakami. Very low bit-rate video coding with block partitioning and adaptive selection of two time-differential frame memories. IEEE Transactions on Circuits and Systems for Video Technology, 7(1):212-220, February 1997.

[FG01a] M. Flierl and B. Girod. Further investigation of multihypothesis motion pictures. Document VCEG-M40, ITU-T Video Coding Experts Group, April 2001. http:// standards. pictel.com/ ftp/ video-site/ 0104_Aus/ VCEGM40.doc.

[FG01b] M. Flierl and B. Girod. Multihypothesis motion-compensated prediction with forward-adaptive hypothesis switching. In Proceedings of the Picture Coding Symposium, pages 195-198, Seoul, Korea, April 2001.

[FG01c] M. Flierl and B. Girod. Multihypothesis motion estimation for video coding. In Proceedings of the Data Compression Conference, pages 341-350, Snowbird, Utah, March 2001.

[FG01d] M. Flierl and B. Girod. Multihypothesis predition for B frames. Document VCEG-N40, ITU-T Video Coding Experts Group, September 2001. http:// standards.pictel.com/ ftp/ video-site/ 0109_San/ VCEG-N40.doc.

[FG02a] M. Flierl and B. Girod. Generalized B pictures. In Proceedings of the Workshop on MPEG-4, San Jose, CA, June 2002.

[FG02b] M. Flierl and B. Girod. Video coding with motion compensation for groups of pictures. In Proceedings of the IEEE International Conference on Image Processing, volume 1, pages 69-72, Rochester, NY, September 2002.

[FG03] M. Flierl and B. Girod. Investigation of motion-compensated lifted wavelet transforms. In Proceedings of the Picture Coding Symposium, Saint-Malo, France, April 2003.

[Fli97a] M. Flierl. Bewegungskompensierte Multihypothesen-Langzeitprädiktion. Diploma thesis, Telecommunications Laboratory, University of ErlangenNuremberg, Erlangen, Germany, September 1997.

[Fli97b] M. Flierl. Untersuchung von Algorithmen zur Bestimmung von QuadtreeStrukturen bei der Videocodierung mit Blöcken variabler Größe. Project thesis, Telecommunications Laboratory, University of Erlangen-Nuremberg, Erlangen, Germany, March 1997.

[FPP00] B. Felts and B. Pesquet-Popescu. Efficient context modeling in scalable 3D wavelet-based video compression. In Proceedings of the IEEE International Conference on Image Processing, pages 1004-1007, Vancouver, Canada, September 2000. 
[FVC97] E. Francois, J.-F. Vial, and B. Chupeau. Coding algorithm with region-based motion compensation. IEEE Transactions on Circuits and Systems for Video Technology, 7(1):97-108, February 1997.

[FWG98] M. Flierl, T. Wiegand, and B. Girod. A locally optimal design algorithm for block-based multi-hypothesis motion-compensated prediction. In Proceedings of the Data Compression Conference, pages 239-248, Snowbird, Utah, April 1998.

[FWG00a] M. Flierl, T. Wiegand, and B. Girod. Rate-constrained multi-hypothesis motion-compensated prediction for video coding. In Proceedings of the IEEE International Conference on Image Processing, volume 3, pages 150-153, Vancouver, Canada, September 2000.

[FWG00b] M. Flierl, T. Wiegand, and B. Girod. A video codec incorporating blockbased multi-hypothesis motion-compensated prediction. In Proceedings of the SPIE Conference on Visual Communications and Image Processing, volume 4067, pages 238-249, Perth, Australia, June 2000.

[FWG01] M. Flierl, T. Wiegand, and B. Girod. Multihypothesis pictures for H.26L. In Proceedings of the IEEE International Conference on Image Processing, volume 3, pages 526-529, Thessaloniki, Greece, October 2001.

[FWG02] M. Flierl, T. Wiegand, and B. Girod. Rate-constrained multihypothesis prediction for motion compensated video compression. IEEE Transactions on Circuits and Systems for Video Technology, 12:957-969, November 2002.

[Ger90] A. Gersho. Optimal nonlinear interpolative vector quantization. IEEE Transactions on Communications, 38(9):1285-1287, September 1990.

[GF02] B. Girod and M. Flierl. Multi-frame motion-compensated video compression for the digital set-top box. In Proceedings of the IEEE International Conference on Image Processing, volume 2, pages 1-4, Rochester, NY, September 2002 .

[GG92] A. Gersho and R.M. Gray. Vector Quantization and Signal Compression. Kluwer Academic Press, 1992.

[Gir87] B. Girod. The efficiency of motion-compensating prediction for hybrid coding of video sequences. IEEE Journal on Selected Areas in Communications, SAC-5(7):1140-1154, August 1987.

[Gir93a] B. Girod. Motion-compensating prediction with fractional-pel accuracy. IEEE Transactions on Communications, 41(4):604-612, April 1993.

[Gir93b] B. Girod. Motion compensation: Visual aspects, accuracy, and fundamental limits. In M.I. Sezan and R.L. Lagendijk, editors, Motion Analysis And Image Sequence Processing. Kluwer Academic Publishers, Boston, 1993. 
[Gir94] B. Girod. Rate-constrained motion estimation. In Proceedings of the SPIE Conference on Visual Communications and Image Processing, pages 10261034, Chicago, September 1994.

[Gir98] B. Girod. Why b-pictures work: a theory of multi-hypothesis motioncompensated prediction. In Proceedings of the IEEE International Conference on Image Processing, pages 213-217, Chicago, IL, October 1998.

[Gir00] B. Girod. Efficiency analysis of multihypothesis motion-compensated prediction for video coding. IEEE Transactions on Image Processing, 9(2):173-183, February 2000.

[GM86] B. Girod and T. Micke. Efficiency of motion-compensating prediction in a generalized hybrid coding scheme. In Proceedings of the Picture Coding Symposium, pages 85-86, Tokyo, Japan, April 1986.

[GO96] O.G. Guleryuz and M.T. Orchard. Rate-distortion based temporal filtering for video compression. In Proceedings of the Data Compression Conference, pages 122-131, Snowbird, UT, March 1996.

[GO01] O.G. Guleryuz and M.T. Orchard. On the DPCM compression of Gaussian autoregressive sequenzes. IEEE Transactions on Information Theory, 47(3):945-956, March 2001.

[Gra84] R.M. Gray. Vector quantization. IEEE Acoustics, Speech, and Signal Processing Magazine, 1:4-29, April 1984.

[GV93] M. Gothe and J. Vaisey. Improving motion compensation using multiple temporal frames. In Proceedings of the IEEE Pacific Rim Conference on Communications, Computers, and Signal Processing, pages 157-160, Victoria, BC, May 1993.

[GWS $\left.{ }^{+} 98\right]$ B. Girod, T. Wiegand, E. Steinbach, M. Flierl, and X. Zhang. High-order motion compensation for low bit-rate video. In Proceedings of the European Signal Processing Conference, pages 253-256, Island of Rhodes, Greece, September 1998.

[Han00] M. Hannuksela. Prediction from temporally subsequent pictures. Document Q15-K38, ITU-T Video Coding Experts Group, August 2000. http:// standards.pictel.com/ ftp/ video-site/ 0008_Por/ q15k38.doc.

[Hep90] D. Hepper. Efficiency analysis and application of uncovered background prediction in a low bit rate image coder. IEEE Transactions on Communications, 38(9):1578-1584, September 1990.

[HP90] B.G. Haskell and A. Puri. Conditional motion compensated interpolation of digital motion video. United States Patent 4958 226, September 1990. Filed: September 27, 1989. 
[HW97] S.-C. Han and J.W. Woods. Spatiotemporal subband/wavelet coding of video with object-based motion information. In Proceedings of the IEEE International Conference on Image Processing, pages 629-632, Santa Barbara, CA, October 1997.

[HW98] S.-C. Han and J.W. Woods. Adaptive coding of moving objects for very low bit rates. IEEE Journal on Selected Areas in Communications, 16(1):56-70, January 1998.

[ISO96] ISO/IEC. 13818-2 Information Technology - Generic Coding of Moving Pictures and Associated Audio Information: Video (MPEG-2), 1996.

[ITU98a] ITU-T. Recommendation H.263, Version 2 (Video Coding for Low Bitrate Communication), 1998.

[ITU98b] ITU-T Video Coding Experts Group. Video Codec Test Model, Near Term, Version 10 (TMN-10), Draft 1, Q15-D65, April 1998. http:// standards.pictel.com/ ftp/ video-site/ 9804_Tam/ q15d65.doc.

[ITU00] ITU-T. Recommendation H.263++ (Video Coding for Low Bitrate Communication), 2000.

[ITU01] ITU-T Video Coding Experts Group. H.26L Test Model Long Term Number 9, TML-9, December 2001. http:// standards.pictel.com/ ftp/ video-site/ h26L/ tml9.doc.

[ITU02] ITU-T Video Coding Experts Group and ISO/IEC Moving Picture Experts Group. Working Draft Number 2, Revision 7, April 2002. ftp:// ftp.imtc-files.org/ c:/ inetpub/ ftpsites/ imtc/ jvt-experts/ draft_standard/ jwd2r7.zip.

[JJ81] J.R. Jain and A.K. Jain. Displacement measurement and its application in interframe image coding. IEEE Transactions on Communications, 29(12):1799-1808, December 1981.

[JN84] N.S. Jayant and P. Noll. Digital Coding of Waveforms. Prentice-Hall, Inc., Englewood Cliffs, NJ, 1984.

[JP01] B. Jeon and Y. Park. Mode decision for B pictures in TML-5. Document VCEG-L10, ITU-T Video Coding Experts Group, January 2001. http:// standards.pictel.com/ ftp/ video-site/ 0101_Eib/ VCEG-L10.doc.

[KCS99] F. Kossentini, W.C. Chung, and M.J.T. Smith. Rate-distortion-constrained subband video coding. IEEE Transactions on Image Processing, 8(2):145154, February 1999. 
[KK94] M.-K. Kim and J.-K. Kim. Efficient motion estimation algorithm for bidirectional prediction scheme. IEE Electronics Letters, 30(8):632-633, April 1994.

[KKS01] S. Kondo, S. Kadono, and M. Schlockermann. New prediction method to improve B-picture coding efficiency. Document VCEG-O26, ITU-T Video Coding Experts Group, December 2001. http:// standards.pictel.com/ ftp/ video-site/ 0112_Pat/ VCEG-O26.doc.

[KLSW97] F. Kossentini, Y.-W. Lee, M.J.T. Smith, and R.K. Ward. Predictive RD optimized motion estimation for very low bit-rate video coding. IEEE Journal on Selected Areas in Communications, 15(9):1752-1763, December 1997.

[Kna76] S.C. Knauer. Real-time video compression algorithm for Hadamard transform processing. IEEE Transactions on Electromagnetic Compatibility, EMC-18:28-36, February 1976.

[KO95] J. Katto and M. Ohta. An analytical framework for overlapped motion compensation. In Proceedings of the IEEE International Conference on Acoustics, Speech and Signal Processing, volume 5, pages 2189-2192, 1995.

[KP97] B.-J. Kim and W.A. Pearlman. An embedded wavelet video coder using three-dimensional set partitioning in hierarchical trees (SPIHT). In Proceedings of the Data Compression Conference, pages 251-260, Snowbird, UT, March 1997.

[Kro89] T. Kronander. Motion compensated 3-dimensional wave-form image coding. In Proceedings of the IEEE International Conference on Acoustics, Speech and Signal Processing, volume 3, pages 1921-1924, Glasgow, Scotland, May 1989.

[Kro90] T. Kronander. New results on 3-dimensional motion compensated subband coding. In Proceedings of the Picture Coding Symposium, pages 8.5-1, Cambridge, MA, March 1990.

[KSY95] J. Kovačević, R.J. Safranek, and E.M. Yeh. Adaptive bidirectional timerecursive interpolation for deinterlacing. In Proceedings of the Data Compression Conference, page 446, Snowbird, UT, March 1995.

[KSY97] J. Kovačević, R.J. Safranek, and E.M. Yeh. Deinterlacing by successive approximation. IEEE Transactions on Image Processing, 6(2):339-344, February 1997.

[KV88] G. Karlsson and M. Vetterli. Three dimensional sub-band coding of video. In Proceedings of the IEEE International Conference on Acoustics, Speech and Signal Processing, pages 1100-1103, New York, NY, April 1988. 
[KW97] J. Kim and J.W. Woods. Spatio-temporal adaptive 3-d kalman filter for video. IEEE Transactions on Image Processing, 6(3):414-424, March 1997.

[KW98] J. Kim and J.W. Woods. 3-d kalman filter for image motion estimation. IEEE Transactions on Image Processing, 7(1):42-52, January 1998.

[KWM99] R. Krishnamurthy, J.W. Woods, and P. Moulin. Frame interpolation and bidirectional prediction of video using compactly encoded optical-flow fields and label fields. IEEE Transactions on Circuits and Systems for Video Technology, 9(5):713-726, August 1999.

[KXP00] B.-J. Kim, Z. Xiong, and W.A. Pearlman. Low bit-rate scalable video coding with 3-D set partitioning in hierarchical trees (3-D SPIHT). IEEE Transactions on Circuits and Systems for Video Technology, 10(8):1374-1387, December 2000 .

[LD94] J. Lee and B.W. Dickinson. Joint optimization of frame type selection and bit allocation for MPEG video encoders. In Proceedings of the IEEE International Conference on Image Processing, volume 2, pages 962-966, Austin, TX, November 1994.

[Lee95] J. Lee. Optimal quadtree for variable block size motion estimation. In Proceedings of the IEEE International Conference on Image Processing, pages 480-483, Washington, DC, October 1995.

[LF95] H. Li and R. Forchheimer. A transformed block-based motion compensation technique. IEEE Transactions on Communications, 43(2/3/4):1673-1676, February 1995.

[LG94] A. Leon-Garcia. Probability and Random Processes for Electrical Engineering. Addison-Wesley Publishing Company, Inc., Reading, Massachusetts, 1994.

[LG96] X. Li and C. Gonzales. A locally quadratic model of the motion estimation error criterion function and its application to subpixel interpolations. IEEE Transactions on Circuits and Systems for Video Technology, 6(1):118-122, February 1996.

[Lig95] M. Lightstone. Efficient Variable Rate Image and Video Coding in an Entropy-Constrained Framework. PhD thesis, University of California Santa Barbara, August 1995.

[Lil99] K. Lillevold. B pictures in H.26L. Document Q15-I08, ITU-T Video Coding Experts Group, October 1999. http:// standards.pictel.com/ ftp/ video-site/ 9910_Red/ q15i08.doc. 
[Lil00] K. Lillevold. Improved direct mode for B pictures in TML. Document Q15-K44, ITU-T Video Coding Experts Group, August 2000. http:// standards.pictel.com/ ftp/ video-site/0008_Por/ q15k44.doc.

[LL91] F. Lavagetto and R. Leonardi. Block adaptive quantization of multiple frame motion field. In Proceedings of the SPIE Conference on Visual Communications and Image Processing, volume 1605, pages 534-543, Boston, MA, November 1991.

$\left[\mathrm{LLL}^{+} 01\right] \quad$ L. Luo, J. Li, S. Li, Z. Zhuang, and Y.-Q. Zhang. Motion-compensated lifting wavelet and its application in video coding. In Proceedings of the IEEE International Conference on Multimedia and Expo, pages 481-484, Tokyo, Japan, August 2001.

[LS95] W. Li and E. Salari. Successive elimination algorithm for motion estimation. IEEE Transactions on Image Processing, 4(1):105-107, January 1995.

[LT97] Y. Lin and S. Tai. Fast full-search block matching algorithm for motioncompensated video compression. IEEE Transactions on Communications, 45(5):527-531, May 1997.

[LW95] Y.Y. Lee and J.W. Woods. Motion vector quantization for video coding. IEEE Transactions on Image Processing, 4(3):378-382, March 1995.

[LWLZ02] L. Luo, Y. Wu, J. Li, and Y.-Q. Zhang. 3-D wavelet compression and progressive inverse wavelet synthesis rendering of concentric mosaic. IEEE Transactions on Image Processing, 11(7):802-816, July 2002.

[Lyn92] W.E. Lynch. Bidirectional motion estimation based on P frame motion vectors and area overlap. In Proceedings of the IEEE International Conference on Acoustics, Speech and Signal Processing, volume 3, pages 445-448, San Francisco, CA, March 1992.

[Mal89] S.G. Mallat. A theory for multiresolution signal decomposition: the wavelet representation. IEEE Transactions on Pattern Analysis and Machine Intelligence, 11(7):674-693, July 1989.

[MBHW01] D. Marpe, G. Blättermann, G. Heising, and T. Wiegand. Further results for CABAC entropy coding scheme. Document VCEG-M59, ITU-T Video Coding Experts Group, April 2001. http:// standards.pictel.com/ ftp/ videosite/ 0104_Aus/ VCEG-M59.doc.

[MBW01] D. Marpe, G. Blättermann, and T. Wiegand. Adaptive codes for H.26L. Document VCEG-L13, ITU-T Video Coding Experts Group, January 2001. http:// standards.pictel.com/ ftp/ video-site/ 0101_Eib/ VCEG-L13.doc. 
[MC99] D. Marpe and H.L. Cycon. Very low bit-rate video coding using waveletbased techniques. IEEE Transactions on Circuits and Systems for Video Technology, 9(1):85-94, February 1999.

[MF98] N. Merhav and M. Feder. Universal prediction. IEEE Transactions on Information Theory, 44(6):2124-2147, October 1998.

[MFRT94] R.M. Mersereau, S.H. Fatemi, C.H. Richardson, and K.K. Truong. Methods for low bit-rate video compression: Some issues and answers. In Proceedings of the SPIE Conference on Visual Communications and Image Processing, pages $2-13,1994$.

[Mic86] T. Micke. Vergleich eines prädiktiven und eines interpolativen bewegungskompensierenden Codierverfahrens für Fersehbildsignale. Diploma thesis, Institut für Theoretische Nachrichtentechnik und Informationsverarbeitung, Universität Hannover, Hannover, Germany, 1986.

[MK85] N. Mukawa and H. Kuroda. Uncovered background prediction in interframe coding. IEEE Transactions on Communications, COM-33(11):1227-1231, November 1985.

[MKW97] P. Moulin, R. Krishnamurthy, and J.W. Woods. Multiscale modeling and estimation of motion fields for video coding. IEEE Transactions on Image Processing, 6(12):1606-1620, December 1997.

[MPG85] H.G. Musmann, P. Pirsch, and H.J. Grallert. Advances in picture coding. Proceedings of the IEEE, 73(4):523-548, April 1985.

[MXWM01] G. Minami, Z. Xiong, A. Wang, and S. Mehrotra. 3-D wavelet coding of video with arbitrary regions of support. IEEE Transactions on Circuits and Systems for Video Technology, 11(9):1063-1068, September 2001.

[NBWL95] T. Naveen, F. Bosveld, J.W. Woods, and R.L. Lagendijk. Rate constrained multiresolution transmission of video. IEEE Transactions on Circuits and Systems for Video Technology, 5(3):193-206, June 1995.

[NH94] Y. Nakaya and H. Harashima. Motion compensation based on spatial transformations. IEEE Transactions on Circuits and Systems for Video Technology, 4(3):339-356, June 1994.

[NO82] Y. Ninomiya and Y. Ohtsuka. A motion-compensated interframe coding scheme for television pictures. IEEE Transactions on Communications, COM-30(1):201-211, January 1982.

[NO92] S. Nogaki and M. Ohta. An overlapped block motion compensation for high quality motion picture coding. In Proceedings of the IEEE International Symposium on Circuits and Systems, pages 184-187, May 1992. 
[NR83] A.N. Netravali and J.D. Robbins. Video signal interpolation using motion estimation. United States Patent 4383 272, May 1983. Filed: April 13, 1981.

[Ohm92] J.-R. Ohm. Temporal domain sub-band video coding with motion compensation. In Proceedings of the IEEE International Conference on Acoustics, Speech and Signal Processing, volume 3, pages 229-232, San Francisco, CA, March 1992.

[Ohm93] J.-R. Ohm. Advanced packet-video coding based on layered VQ and SBC techniques. IEEE Transactions on Circuits and Systems for Video Technology, 3(3):208-221, June 1993.

[Ohm94a] J.-R. Ohm. Motion-compensated 3-d subband coding with multiresolution representation of motion parameters. In Proceedings of the IEEE International Conference on Image Processing, volume 3, pages 250-254, Austin, TX, November 1994.

[Ohm94b] J.-R. Ohm. Three-dimensional subband coding with motion compensation. IEEE Transactions on Circuits and Systems for Video Technology, 3(5):559571, September 1994.

[Ohm02] J.-R. Ohm. Motion-compensated wavelet lifting filters with flexible adaptation. In Proceedings of the International Workshop on Digital Communications, pages 113-120, Capri, Italy, September 2002.

[OR97] J.-R. Ohm and K. Rümmler. Variable-raster multiresolution video processing with motion compensation techniques. In Proceedings of the IEEE International Conference on Image Processing, pages 759-762, Santa Barbara, CA, USA, October 1997.

[OS94] M.T. Orchard and G.J. Sullivan. Overlapped block motion compensation: An estimation-theoretic approach. IEEE Transactions on Image Processing, 3(5):693-699, September 1994.

[PA90] A. Puri and R. Aravind. On comparing motion-interpolating structures for video coding. In Proceedings of the SPIE Conference on Visual Communications and Image Processing, pages 1560-1571, Lausanne, Switzerland, October 1990.

[PA91] A. Puri and R. Aravind. Motion-compensated video coding with adaptive perceptual quantization. IEEE Transactions on Circuits and Systems for Video Technology, 1(4):351-361, December 1991.

[PAB01] C. Parisot, M. Antonini, and M. Barlaud. 3D scan based wavelet transform for video coding. In Proceedings of the IEEE Workshop on Multimedia Signal Processing, pages 403-408, Cannes, France, October 2001. 
[PAB02] C. Parisot, M. Antonini, and M. Barlaud. Motion-compensated scan based wavelet transform for video coding. In Proceedings of the International Workshop on Digital Communications, pages 121-127, Capri, Italy, September 2002 .

[PAH93] A. Puri, R. Aravind, and B. Haskell. Adaptive frame/field motion compensated video coding. Signal Processing: Image Communication, 5(1-2):39-58, February 1993.

[PAHL90] A. Puri, R. Aravind, B.G. Haskell, and R. Leonardi. Video coding with motion-compensated interpolation for CD-ROM applications. Signal Processing: Image Communication, 2(2):127-144, August 1990.

[Pap91] A. Papoulis. Probability, Random Variables, and Stochastic Processes. McGraw-Hill, New York, 1991.

[PJF95] C.I. Podilchuk, N.S. Jayant, and N. Farvardin. Three-dimensional subband coding of video. IEEE Transactions on Image Processing, 4(2):125-139, February 1995.

[PMGF96] W.B. Pennebaker, J.L. Mitchell, D. Le Gall, and C. Fogg. MPEG Video Compression Standard. Kluwer Academic Publishers, Boston, 1996.

[PPB01] B. Pesquet-Popescu and V. Bottreau. Three-dimensional lifting schemes for motion compensated video compression. In Proceedings of the IEEE International Conference on Acoustics, Speech and Signal Processing, volume 3, pages 1793-1796, Salt Lake City, UT, May 2001.

[PT94] K. Pang and T. Tan. Optimum loop filter in hybrid coders. IEEE Transactions on Circuits and Systems for Video Technology, 4(2):158-167, April 1994.

[Pur88] Atul Puri. Efficient Motion-Compensated Coding for Low Bit-Rate Video Applications. PhD thesis, The City University of New York, 1988.

[PYH94] A. Puri, L. Yan, and B.G. Haskell. Temporal resolution scalable video coding. In Proceedings of the IEEE International Conference on Image Processing, pages 947-951, Austin, TX, November 1994.

[RCN95] J. Ribas-Corbera and D.L. Neuhoff. Optimal bit allocations for lossless video coders: Motion vectors vs. difference frames. In Proceedings of the IEEE International Conference on Image Processing, pages 180-183, Washington, DC, October 1995.

[RCN97] J. Ribas-Corbera and D. L. Neuhoff. On the optimal block size for blockbased, motion-compensated video coders. In Proceedings of the SPIE Conference on Visual Communications and Image Processing, volume 2, pages 1132-1143, San Jose, CA, January 1997. 
[RCN01] J. Ribas-Corbera and D. L. Neuhoff. Optimizing motion-vector accuracy in block-based video coding. IEEE Transactions on Circuits and Systems for Video Technology, 11(4):497-511, April 2001.

[RCS92] J. Ribas-Corbera and J. Sklansky. Interpolation of cinematic sequences. In Proceedings of the IEEE Workshop on Applications of Computer Vision, pages 36-44, November 1992.

[RFG01] P. Ramanathan, M. Flierl, and B. Girod. Multi-hypothesis disparitycompensated light field compression. In Proceedings of the IEEE International Conference on Image Processing, volume 2, pages 101-104, Thessaloniki, Greece, October 2001.

[ROV94] K. Ramchandran, A. Ortega, and M. Vetterli. Bit allocation for dependent quantization with applications to multiresolution and MPEG video coders. IEEE Transactions on Image Processing, 3(5):533-545, September 1994.

[RV93] K. Ramchandran and M. Vetterli. Best wavelet packet bases in a ratedistortion sense. IEEE Transactions on Image Processing, 2(2):160-174, April 1993.

[SB91a] G.J. Sullivan and R.L. Baker. Motion compensation for video compression using control grid interpolation. In Proceedings of the IEEE International Conference on Acoustics, Speech and Signal Processing, pages 2713-2716, Toronto, Canada, May 1991.

[SB91b] G.J. Sullivan and R.L. Baker. Rate-distortion optimized motion compensation for video compression using fixed or variable size blocks. In Proceedings of the IEEE Global Telecommunications Conference, volume 3, pages 85-90, Phoenix, AZ, December 1991.

[SB94] G.J. Sullivan and R.L. Baker. Efficient quadtree coding of images and video. IEEE Transactions on Image Processing, 3:327-331, May 1994.

[SC89] M. Soryani and R.J. Clarke. Image segmentation and motion-adaptive frame interpolation for coding moving sequences. In Proceedings of the IEEE International Conference on Acoustics, Speech and Signal Processing, pages 1882-1885, Glasgow, Scotland, May 1989.

[SG86] M.J. Sabin and R.M. Gray. Global convergence and empirical consistency of the generalized lloyd algorithm. IEEE Transactions on Information Theory, 32(2):148-155, March 1986.

[SG88] Y. Shoham and A. Gersho. Efficient bit allocation for an arbitrary set of quantizers. IEEE Transactions on Acoustics, Speech and Signal Processing, 36(9):1445-1453, September 1988. 
[SG01] T. Shanableh and M. Ghanbari. The improtance of the bi-directionally predicted pictures in video streaming. IEEE Transactions on Circuits and Systems for Video Technology, 11(3):402-414, March 2001.

[Sha93] J.M. Shapiro. Embedded image coding using zerotrees of wavelet coefficients. IEEE Transactions on Signal Processing, 41(12):3445-3462, December 1993.

[SK96] G.M. Schuster and A.K. Katsaggelos. A video compression scheme with optimal bit allocation between displacement vector field and displaced frame difference. In Proceedings of the IEEE International Conference on Acoustics, Speech and Signal Processing, pages 1966-1969, Atlanta, GA, May 1996.

[SK97] G.M. Schuster and A.K. Katsaggelos. A video compression scheme with optimal bit allocation among segmentation, motion, and residual error. IEEE Transactions on Image Processing, 6(11):1487-1501, November 1997.

[SO94] G.J. Sullivan and M.T. Orchard. Methods of reduced-comlexity overlapped block motion compensation. In Proceedings of the IEEE International Conference on Image Processing, volume 2, pages 957-961, Austin, TX, November 1994.

[SO01] T. Stockhammer and T. Oelbaum. Coding results for CABAC entropy coding scheme. Document VCEG-M54, ITU-T Video Coding Experts Group, April 2001. http:// standards.pictel.com/ ftp/ video-site/ 0104_Aus/ VCEGM54.doc.

[SP96] A. Said and W.A. Pearlman. A new, fast, and efficient image codec based on set partitioning in hierarchical trees. IEEE Transactions on Circuits and Systems for Video Technology, 6(3):243-250, June 1996.

[ST01] A. Secker and D. Taubman. Motion-compensated highly scalable video compression using an adaptive 3D wavelet transform based on lifting. In Proceedings of the IEEE International Conference on Image Processing, volume 2, pages 1029-1032, Thessaloniki, Greece, October 2001.

[ST02] A. Secker and D. Taubman. Highly scalable video compression using a liftingbased 3D wavelet transform with deformable mesh motion compensation. In Proceedings of the IEEE International Conference on Image Processing, volume 3, pages 749-752, Rochester, NY, September 2002.

[Str90] P. Strobach. Tree-structured scene adaptive coder. IEEE Transactions on Communications, 38(4):477-486, April 1990.

[Su193] G.J. Sullivan. Multi-hypothesis motion compensation for low bit-rate video coding. In Proceedings of the IEEE International Conference on Acoustics, Speech and Signal Processing, volume 5, pages 437-440, Minneapolis, April 1993. 
[SW98] G.J. Sullivan and T. Wiegand. Rate-distortion optimization for video compression. IEEE Signal Processing Magazine, 15:74-90, November 1998.

[SW01] H. Schwarz and T. Wiegand. An improved H.26L coder using lagrangian coder control. Document VCEG-HHI, ITU-T Video Coding Experts Group, May 2001. http:// standards.pictel.com/ ftp/ video-site/ 0105_Por/ HHIRDOpt.doc.

[Swe95] W. Sweldens. The lifting scheme: A new philosophy in biorthogonal wavelet constructions. In Wavelet Applications in Signal and Image Processing III, pages 68-79. SPIE 2569, 1995.

[Swe98] W. Sweldens. The lifting scheme: A construction of second generation wavelets. SIAM Journal on Mathematical Analysis, 29(2):511-546, 1998.

[TA97] C.-W. Tang and O.C. Au. Unidirectional motion compensated temporal interpolation. In Proceedings of the IEEE International Symposium on Circuits and Systems, pages 1444-1447, Hong Kong, June 1997.

[TA98] C.-W. Tang and O.C. Au. Comparison between block-based and pixel-based temporal interpolation for video coding. In Proceedings of the IEEE International Symposium on Circuits and Systems, pages 122-125, Monterey, CA, June 1998.

[TAL02] A.M. Tourapis, O.C. Au, and M.L. Liou. Highly efficient predictive zonal algorithms for fast block-matching motion estimation. IEEE Transactions on Circuits and Systems for Video Technology, 12(10):934-947, October 2002.

[Tau94] D. Taubman. Directionality and Scalability in Image and Video Compression. PhD thesis, University of California, Berkeley, 1994.

[Tau00] D. Taubman. High performance scalable image compression with EBCOT. IEEE Transactions on Image Processing, 9(7):1158-1170, July 2000.

[TB89] R. Thoma and M. Bierling. Motion compensating interpolation considering covered and uncovered background. Signal Processing: Image Communication, 1(2):191-212, October 1989.

[TCAL01] A.M. Tourapis, H.-Y. Cheong, O.C. Au, and M.L. Liou. N-dimensional zonal algorithms. the future of block based motion estimation? In Proceedings of the IEEE International Conference on Image Processing, pages 506-509, Thessaloniki, Greece, October 2001.

[TCLA01] A.M. Tourapis, H.-Y. Cheong, M.L. Liou, and O.C. Au. Temporal interpolation of video sequences using zonal based algorithms. In Proceedings of the IEEE International Conference on Image Processing, pages 895-898, Thessaloniki, Greece, October 2001. 
[Tek95] A.M. Tekalp. Digital Video Processing. Prentice Hall, London, 1995.

[TM01] D.S. Taubman and M.W. Marcellin. JPEG2000: Image Compression Fundamentals, Standards, and Practice. Kluwer Academic Publishers, 2001.

[TO97] B. Tao and M.T. Orchard. Window design for overlapped block motion compensation through statistical motion modeling. In Thirty-First Asilomar Conference on Signals, Systems, and Computers, volume 1, pages 372-376, November 1997.

[TO98] B. Tao and M.T. Orchard. Non-iterative motion estimation for overlapped block motion compensation. In Proceedings of the SPIE Conference on Visual Communications and Image Processing, pages 1032-1040, San Jose, CA, January 1998.

[TO01a] B. Tao and M.T. Orchard. Gradient-based residual variance modeling and its applications to motion-compensated video coding. IEEE Transactions on Image Processing, 10(1):24-35, January 2001.

[TO01b] B. Tao and M.T. Orchard. A parametric solution for optimal overlapped block motion compensation. IEEE Transactions on Image Processing, 10(3):341-350, March 2001.

[TO01c] B. Tao and M.T. Orchard. Removal of motion uncertainty and quantization noise in motion compensation. IEEE Transactions on Circuits and Systems for Video Technology, 11(1):80-90, January 2001.

[TOD97] B. Tao, M.T. Orchard, and B. Dickinson. Joint application of overlapped block motion compensation and loop filtering for low bit-rate video coding. In Proceedings of the IEEE International Conference on Image Processing, pages 626-629, Santa Barbara, CA, October 1997.

[TRK98] J.Y. Tham, S. Ranganath, and A.A. Kassim. Highly scalable wavelet-based video codec for very low bit-rate environment. IEEE Transactions on Circuits and Systems for Video Technology, 8(4):369-377, August 1998.

[TZ94a] D. Taubman and A. Zakhor. Highly scalable, low-delay video compression. In Proceedings of the IEEE International Conference on Image Processing, pages 740-744, Austin, TX, November 1994.

[TZ94b] D. Taubman and A. Zakhor. Multirate 3-d subband coding of video. IEEE Transactions on Image Processing, 3(5):572-588, September 1994.

[TZ94c] D. Taubman and A. Zakhor. Rate and resolution scalable subband coding of video. In Proceedings of the IEEE International Conference on Acoustics, Speech and Signal Processing, volume 5, pages 493-496, Adelaide, Australia, April 1994. 
[TZ96] D. Taubman and A. Zakhor. A common framework for rate and distortion based scaling of highly scalable compressed video. IEEE Transactions on Circuits and Systems for Video Technology, 6(4):329-354, August 1996.

[UE90] M. Unser and M. Eden. Weighted averaging of a set of noisy images for maximum signal-to-noise ratio. IEEE Transactions on Acoustics, Speech and Signal Processing, 38(5):890-895, May 1990.

[Use96] B. Usevitch. Optimal bit allocation for biorthogonal wavelet coding. In Proceedings of the Data Compression Conference, pages 387-395, Snowbird, UT, March 1996.

[UVL90] K.M. Uz, M. Vetterli, and D. LeGall. A multiresolution approach to motion estimation and interpolation with application to coding of digital HDTV. In Proceedings of the IEEE International Symposium on Circuits and Systems, volume 2, pages 1298-1301, New Orleans, LA, May 1990.

[UVL91] K.M. Uz, M. Vetterli, and D.J. LeGall. Interpolative multiresolution coding of advanced television with compatible subchannels. IEEE Transactions on Circuits and Systems for Video Technology, 1(1):86-99, March 1991.

[VCM95] L. Vandendorpe, L. Cuvelier, and B. Maison. Statistical properties of prediction error images in motion compensated interlaced image coding. In Proceedings of the IEEE International Conference on Image Processing, pages 192-195, Washington, DC, October 1995.

[VGP02] J. Viéron, C. Guillemot, and S. Pateux. Motion compensated 2D+t wavelet analysis for low rate FGS video compression. In Proceedings of the International Workshop on Digital Communications, pages 129-135, Capri, Italy, September 2002.

[VK95] M. Vetterli and J. Kovacevic. Wavelets and Subband Coding. Signal Processing Series. Prentice Hall, Englewood Cliffs, NJ, 1995.

[WA96] C.-K. Wong and O.C. Au. Modified motion compensated temporal frame interpolation for very low bit rate video. In Proceedings of the IEEE International Conference on Acoustics, Speech and Signal Processing, volume 4, pages 2327-2330, Atlanta, GA, May 1996.

[WAT96] C.-K. Wong, O.C. Au, and C.-W. Tang. Motion compensated temporal interpolation with overlapping. In Proceedings of the IEEE International Symposium on Circuits and Systems, pages 608-611, Atlanta, GA, May 1996.

[Wed99] T. Wedi. A time-recursive interpolation filter for motion compensated prediction considering aliasing. In Proceedings of the IEEE International Conference on Image Processing, pages 672-675, Kobe, Japan, October 1999. 
[Wed02] T. Wedi. Adaptive interpolation filter for motion compensated prediction. In Proceedings of the IEEE International Conference on Image Processing, volume 2, pages 509-512, Rochester, NY, September 2002.

[WFG97] T. Wiegand, M. Flierl, and B. Girod. Entropy-constrained design of quadtree video coding schemes. In Proceedings of the International Conference on Image Processing and its Applications, volume 1, pages 36-40, Dublin, Ireland, July 1997.

[WFG98] T. Wiegand, M. Flierl, and B. Girod. Entropy-constrained linear vector prediction for motion-compensated video coding. In Proceedings of the International Symposium on Information Theory, page 409, Cambridge, MA, August 1998.

[WG94] S.-W. Wu and A. Gersho. Joint estimation of forward and backward motion vectors for interpolative prediction of video. IEEE Transactions on Image Processing, 3(5):684-687, September 1994.

[WG01a] T. Wiegand and B. Girod. Lagrange multiplier selection in hybrid video coder control. In Proceedings of the IEEE International Conference on Image Processing, volume 3, pages 542-545, Thessaloniki, Greece, October 2001.

[WG01b] T. Wiegand and B. Girod. Multi-Frame Motion-Compensated Prediction for Video Transmission. Kluwer Academic Publishers, 2001.

[Wie00] T. Wiegand. Multi-Frame Motion-Compensated Prediction for Video Transmission. PhD thesis, University of Erlangen-Nuremberg, Erlangen, Germany, July 2000.

[WL01] J.W. Woods and G. Lilienfield. A resolution and frame-rate scalable subband/wavelet video coder. IEEE Transactions on Circuits and Systems for Video Technology, 11(9):1035-1044, September 2001.

$\left[\mathrm{WLM}^{+} 96\right]$ T. Wiegand, M. Lightstone, D. Mukherjee, T. G. Campbell, and S. K. Mitra. Rate-distortion optimized mode selection for very low bit rate video coding and the emerging H.263 standard. IEEE Transactions on Circuits and Systems for Video Technology, 6(2):182-190, April 1996.

[WN92] J.W. Woods and T. Naveen. A filter based bit allocation scheme for subband compression of HDTV. IEEE Transactions on Image Processing, 1(3):436440, July 1992.

[WOZ02] Y. Wang, J. Ostermann, and Y. Zhang. Video Processing and Communications. Prentice-Hall, Inc., Upper Saddle River, NJ, 2002.

[WS91] H. Watanabe and S. Singhal. Windowed motion compensation. In Proceedings of the SPIE Conference on Visual Communications and Image Processing, pages 582-589, Boston, MA, November 1991. 
[Wu93] Siu-Wai Wu. Enhanced Image and Video Compression with Constraints on the Bit Stream Format. PhD thesis, University of California, Santa Barbara, March 1993.

[WXCM99] A. Wang, Z. Xiong, P.A. Chou, and S. Mehrotra. Three-dimensional wavelet coding of video with global motion compensation. In Proceedings of the Data Compression Conference, pages 404-413, Snowbird, UT, March 1999.

[WZG97a] T. Wiegand, X. Zhang, and B. Girod. Block-based hybrid video coding using motion-compensated long-term memory prediction. In Proceedings of the Picture Coding Symposium, pages 153-158, Berlin, Germany, September 1997.

[WZG97b] T. Wiegand, X. Zhang, and B. Girod. Motion-compensating long-term memory prediction. In Proceedings of the IEEE International Conference on Image Processing, volume 2, pages 17-20, Santa Barbara, CA, USA, October 1997.

[WZG99] T. Wiegand, X. Zhang, and B. Girod. Long-term memory motion-compensated prediction. IEEE Transactions on Circuits and Systems for Video Technology, 9(1):70-84, February 1999.

[XLXZ00] J. Xu, S. Li, Z. Xiong, and Y.-Q. Zhang. Memory-constrained 3D wavelet transforms for video coding without boundary effects. In Proceedings of the IEEE International Symposium on Intelligent Signal Processing and Communication Systems, Honolulu, HI, November 2000.

[XXLZ01] J. Xu, Z. Xiong, S. Li, and Y.-Q. Zhang. Three-dimensional embedded subband coding with optimal truncation (3D ESCOT). Applied and Computational Harmonic Analysis, 10:290-315, 2001.

[XXLZ02] J. Xu, Z. Xiong, S. Li, and Y.-Q. Zhang. Memory-constrained 3D wavelet transform for video coding without boundary effects. IEEE Transactions on Circuits and Systems for Video Technology, 12(9):812-818, September 2002.

[YA92] J. Yonemitsu and B.D. Andrews. Video signal coding method. United States Patent 5155 593, October 1992. Filed: September 27, 1990.

[YLHH00] T. Yang, K. Liang, C. Huang, and K. Huber. Temporal scalability in H.26L. Document Q15-J45, ITU-T Video Coding Experts Group, May 2000. http:// standards.pictel.com/ ftp/ video-site/ 0005_Osa/ q15j45.doc.

[YMO95] Y. Yokoyama, Y. Miyamoto, and M. Ohta. Very low bit rate video coding using arbitrarily shaped region-based motion compensation. IEEE Transactions on Circuits and Systems for Video Technology, 5(6):500-507, December 1995. 
[ZZ92] Y.-Q. Zhang and S. Zafar. Motion-compensated wavelet transform coding for color video compression. IEEE Transactions on Circuits and Systems for Video Technology, 2(3):285-296, September 1992.

[ZZ93] L. Zhao and Z. Zhou. A new algorithm for motion-compensated frame interpolation. In Proceedings of the IEEE International Symposium on Circuits and Systems, pages 9-12, Chicago, Illinois, May 1993.

[ZZJ93] S. Zafar, Y.-Q. Zhang, and B. Jabbari. Multiscale video representation using multiresolution motion compensation and wavelet decomposition. IEEE Journal on Selected Areas in Communications, 11(1):24-35, January 1993. 



\title{
Curriculum Vitae
}

\author{
Name $\quad$ Markus Helmut Flierl \\ Birth July 4, 1971, Eschenbach, Germany \\ Citizenship German
}

Sep. 1977 - Aug. 1983

Sep. 1983 - Aug. 1987

Sep. 1987 - July 1989

Oct. 1989 - Sep. 1990

Oct. 1990 - Sep. 1997

Nov. 1992 - July 1994

Aug. 1994 - May 1995

June 1995 - Aug. 1995

Oct. 1997

Nov. 1997 - Nov. 1998

Dec. 1998

Jan. 1999 - Dec. 2001

until Dec. 2002
Grund- und Hauptschule Auerbach

Staatliche Realschule Pegnitz

Städtische Fachoberschule Nürnberg

Georg-Simon-Ohm Fachhochschule Nürnberg

Friedrich-Alexander-Universität Erlangen-Nürnberg Student in Electrical Engineering

Fellow, Siemens Student Program

Student Assistant, Department of Applied Mathematics

Visiting Student, Florida Institute of Technology, USA

Internship, Siemens, Munich, Germany

Research Assistant, Telecommunications Laboratory, University of Erlangen-Nuremberg

Community Service, Clinical Center, Erlangen

Research Assistant, Telecommunications Laboratory, University of Erlangen-Nuremberg

Scholar, Graduate Research Center, University of Erlangen-Nuremberg

Visiting Researcher, Stanford University, USA 
